\title{
38. RADIOLARIA FROM LEG 27 OF THE DEEP SEA DRILLING PROJECT
}

\author{
G. W. Renz, Scripps Institution of Oceanography, La Jolla, California
}

\section{CONTENTS}

1. Introduction

2. Radiolarians at each site
a. Site 259 with Tables 1 and 2
b. Site 260 with Tables 3 and 4
c. Site 261 with Tables 5 and 6
d. Site 262 with Table 7
e. Site 263 with Table 8

3. Cretaceous stratigraphy

\section{INTRODUCTION}

Radiolarians were recovered in Quaternary deposits at all sites (259-263) and in Cretaceous deposits at all sites except Site 262. The drilling sites are all located in the eastern Indian Ocean (Figure 1).

The occurrence of a very well-preserved sequence of Cretaceous radiolarians consisting of three new assemblages is by far the most interesting and significant aspect of this material. The major portion of this paper will be devoted to this sequence. Although zones have been established for the lower part of the Quaternary in the equatorial Pacific (Nigrini, 1971), the Quaternary samples in this study have been treated as a whole. Those species comprising the assemblages are defined and their abundances noted.

Due to the lack of an established zonation for Cretaceous radiolarians and to the generally poor preservation of samples, range charts for the Cretaceous species encountered in Leg 27 material will not be presented. Since only the one sequence at Site 261, Cores 9-23, appears to be of any biostratigraphic significance, a synchronopticon will be used as a substitute, and perhaps better, method, for presenting and studying the ranges of species in these assemblages.

\section{RADIOLARIANS AT EACH SITE}

Tables 1-8 show the occurrences of radiolarians in the material recovered from Leg 27 . The notations of "A,a"; "C,c"; " $\mathrm{F}, \mathrm{f}$ "; and " $\mathrm{R}, \mathrm{r}$ " indicate that a species is abundant, common, few, or rare with respect to the total number of radiolarians in a sample. The same letters are used to describe the total assemblage. "+" is used to indicate presence where abundance could not be tabulated; "-" indicates the species has been searched for and not found. Assemblage preservation is indicated by " $G$ " (good) where even delicate features are preserved, " $M$ " (moderate) where the more robust characteristics are preserved despite breakage and partial dissolution or recrystallization, and "P" (poor) where nearly all specimens are destroyed or dissolved.
4. Systematics 785

a. Classification outline $\quad 785$

b. Taxonomic Listing (with index) 787

5. Acknowledgments 799

6. References 801

7. Plates 801

a. Synchronopticon (Plates 1-8) 802

b. Others (Plates 9-19) 818

a. Site 259 (Wharton Basin; $29^{\circ} 37^{\prime} \mathrm{S}, 112^{\circ} 42^{\prime} \mathrm{E}$; water depth $4706 \mathrm{~m}$ )

Quaternary Radiolaria range from common to absent in Core 1. Preservation is moderately good but deteriorates rapidly with depth in the core.

There was no recovery of Core 2.

Cores 3 through 30 contain Radiolaria in fluctuating abundances. Preservation is so pobr that few specimens can be identified and none can be used for age determination. Some specimens from Core 8 and lower, however, can be classified on the family or even generic levęl indicating that the material is Cretaceous, i.e., Dicolocapsa spp., Dictyomitra spp., and several Spongodiscidae.

TABLE 1

Cretaceous Radiolarians at Site 259

\begin{tabular}{|c|c|c|c|c|c|c|}
\hline Samples & : & 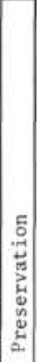 & 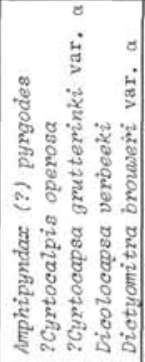 & 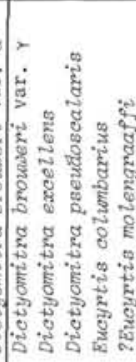 & 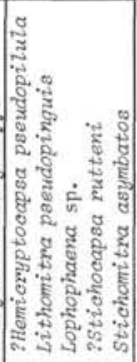 & 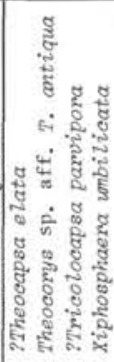 \\
\hline $\begin{array}{l}259-11-3,70-72 \\
259-11-C C \\
259-12-1,70-72 \\
259-12-2,70-72 \\
259-12-3,70-72\end{array}$ & $\begin{array}{l}\mathrm{A} \\
\mathrm{C} \\
\mathrm{A} \\
\mathrm{A} \\
\mathrm{A}\end{array}$ & $\begin{array}{l}\mathrm{p} \\
\mathrm{p} \\
\mathrm{p} \\
\mathrm{p} \\
\mathrm{p}\end{array}$ & $\begin{array}{r}+ \\
+ \\
+ \\
+ \\
+\end{array}$ & +++ & $\begin{array}{lll}+ & & \\
& + & \\
+ & & \\
+ & +\end{array}$ & $\begin{array}{l}+ \\
+ \\
+ \\
+ \\
+ \\
+\end{array}$ \\
\hline $\begin{array}{l}259-12-4,70-72 \\
259-12-5,70-72 \\
259-12-6,118-120 \\
259-12-\mathrm{CC} \\
259-13-1,70-72\end{array}$ & $\begin{array}{l}\mathrm{A} \\
\mathrm{A} \\
\mathrm{A} \\
\mathrm{F} \\
\mathrm{A}\end{array}$ & $\begin{array}{l}\mathrm{p} \\
\mathrm{p} \\
\mathrm{p} \\
\mathrm{p} \\
\mathrm{p}\end{array}$ & $\begin{array}{r}++ \\
+ \\
+ \\
+\end{array}$ & $\begin{array}{l}+++\ldots+ \\
+\end{array}$ & $\frac{++++}{+}$ & $\begin{array}{l}+++ \\
+ \\
+ \\
+\end{array}$ \\
\hline $\begin{array}{l}259-15-C C \\
259-19-1,63-65 \\
259-21-2,70-72 \\
259-25-1,55-57 \\
259-25-2,63-65\end{array}$ & $\begin{array}{l}\mathrm{F} \\
\mathrm{R} \\
\mathrm{F} \\
\mathrm{C} \\
\mathrm{C}\end{array}$ & $\begin{array}{l}\mathrm{p} \\
\mathrm{p} \\
\mathrm{p} \\
\mathrm{p} \\
\mathrm{p}\end{array}$ & $\stackrel{+}{+}$ & + & & + \\
\hline $\begin{array}{l}259-25-3,57-59 \\
259-25-\mathrm{CC}\end{array}$ & $\begin{array}{l}\mathrm{F} \\
\mathrm{C}\end{array}$ & $\begin{array}{l}\mathrm{P} \\
\mathrm{P}\end{array}$ & & + & & + \\
\hline
\end{tabular}




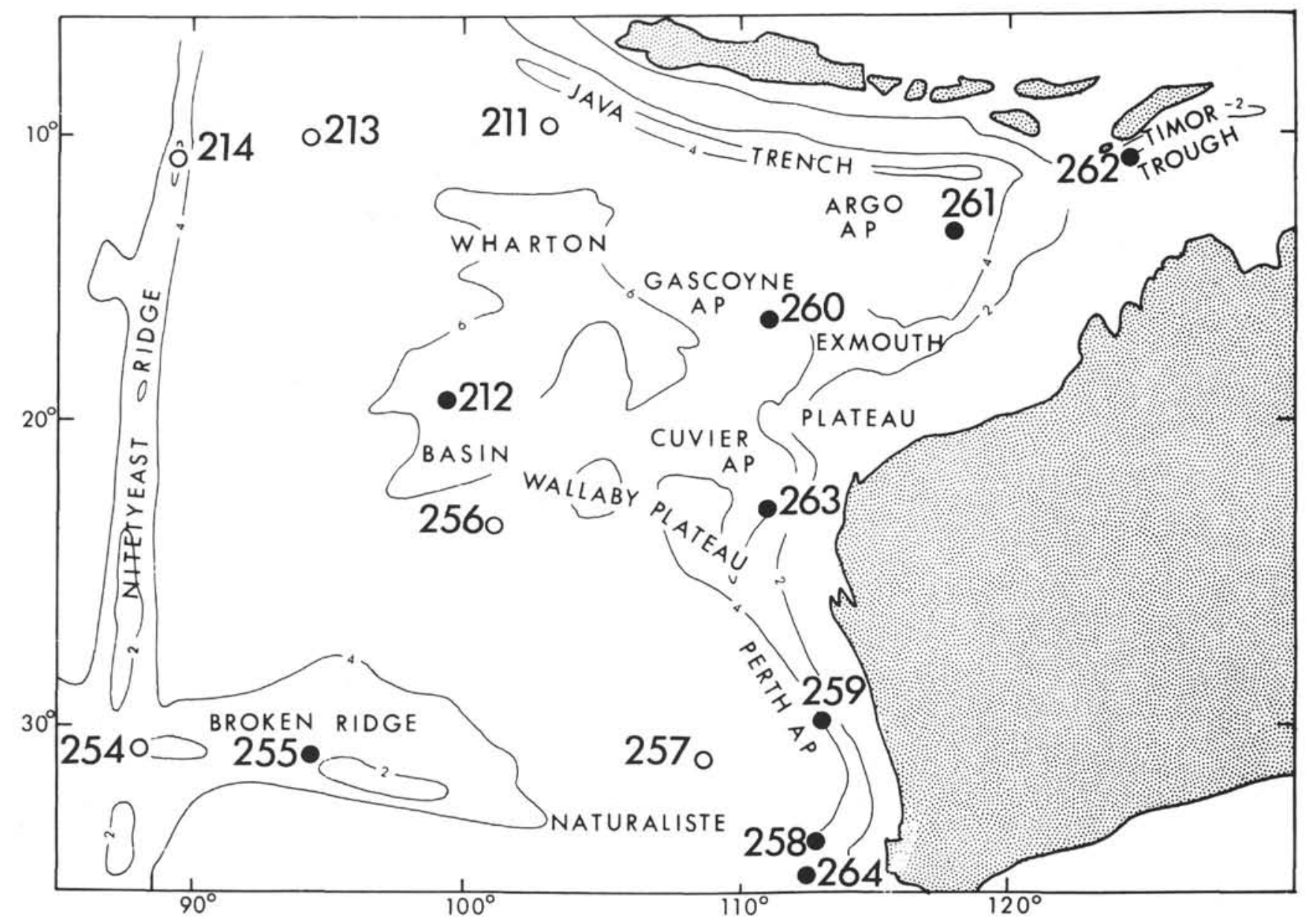

Figure 1. DSDP Leg 27 drilling sites (solid dots) and previous sites (open circles).

TABLE 2

Quaternary Radiolarians at Site 259

\begin{tabular}{|c|c|c|c|c|c|c|c|c|c|c|c|}
\hline Samples & 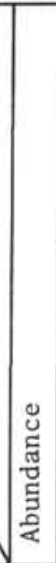 & 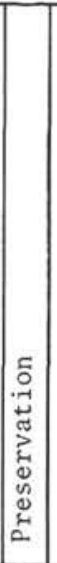 & 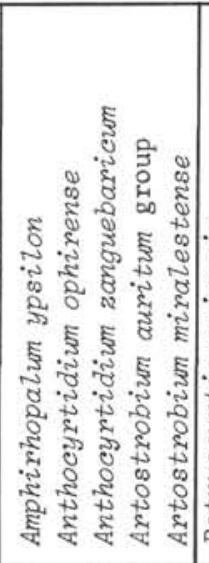 & 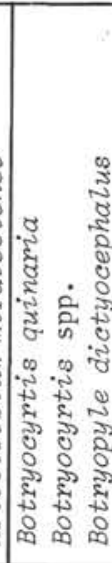 & 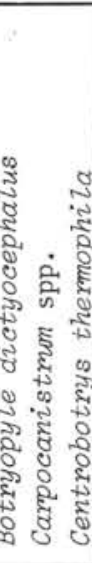 & 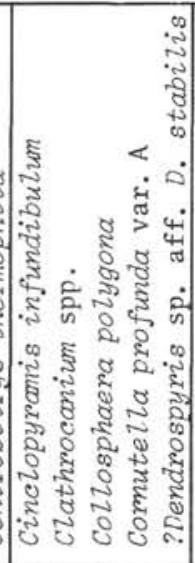 & 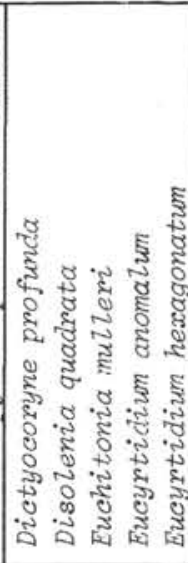 & 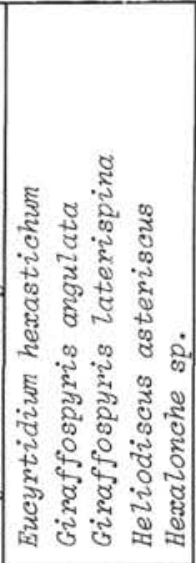 & 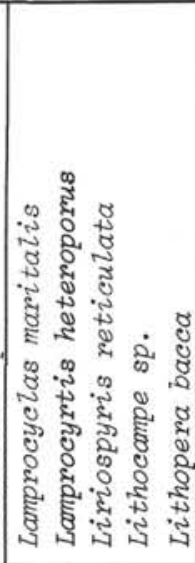 & 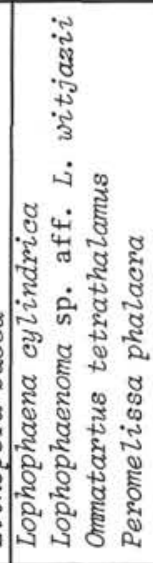 & 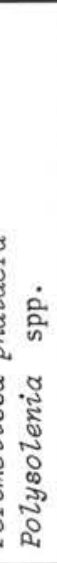 \\
\hline $\begin{array}{ll}259-1-3, & 50-52 \\
259-1-3, & 140-142 \\
259-1-4, & 96-98 \\
259-1-5, & 51-53\end{array}$ & $\begin{array}{l}C \\
F \\
R \\
R\end{array}$ & $\begin{array}{l}M \\
M \\
M \\
M\end{array}$ & 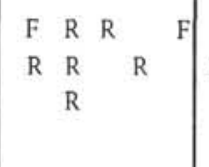 & $R^{R R}$ & $\begin{array}{l}\mathrm{R} \\
\mathrm{F} \\
\mathrm{F} \mathrm{R} \\
\mathrm{R}\end{array}$ & $\begin{array}{lllll}R & R & R & R & R\end{array}$ & $\begin{array}{lll}R & R \\
& F & R\end{array}$ & $\begin{array}{|lllll|}R & R & R & R & F \\
& R & R & R \\
& & R & \end{array}$ & $\begin{array}{llllll}\text { R } & R & R & \text { R } & \text { F } \\
& & & & \text { R } \\
\text { R } & & & & \end{array}$ & $\begin{array}{llll}R & R & C & R \\
& & F\end{array}$ & $\begin{array}{l}F \\
F \\
R \\
R\end{array}$ \\
\hline
\end{tabular}


TABLE 2 - Continued

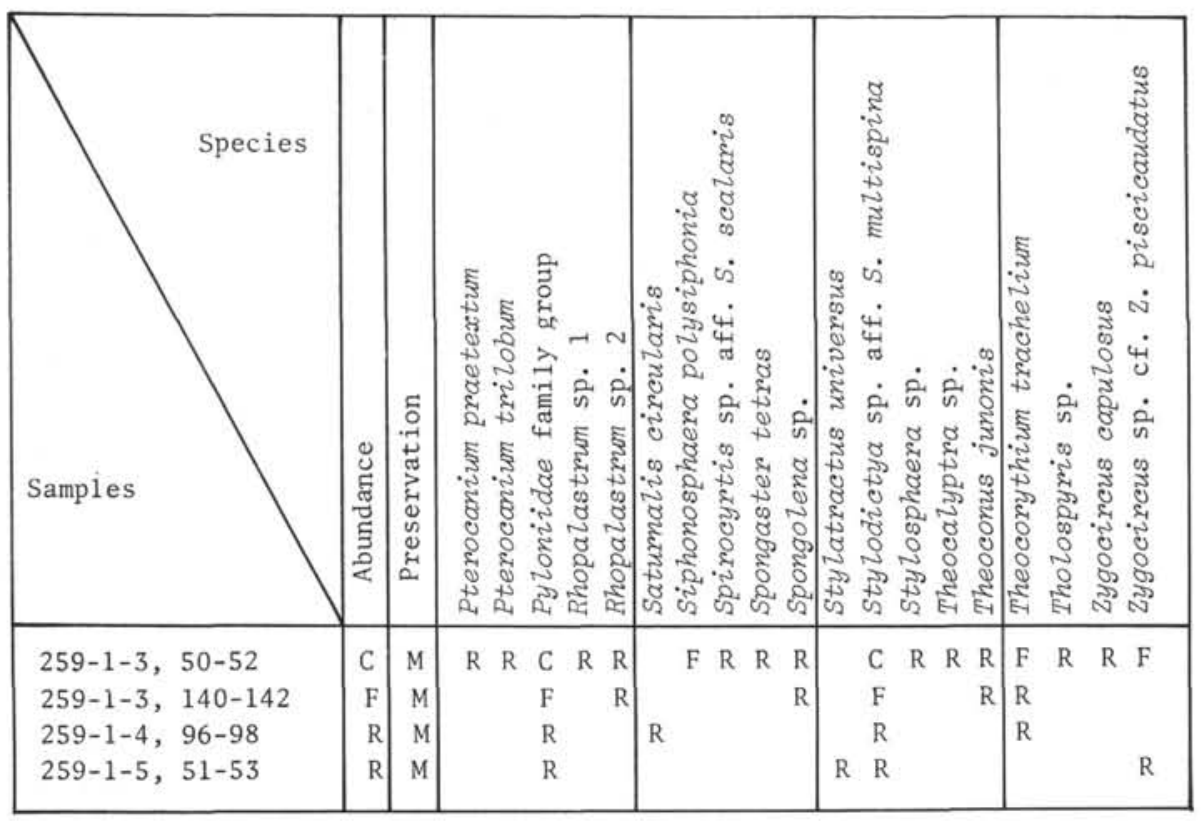

b. Site 260 (Wharton Basin near Exmouth Plateau; $16^{\circ} 09^{\prime} \mathrm{S}, 110^{\circ} 18^{\prime} \mathrm{E}$; water depth $5709 \mathrm{~m}$ )

A typical Quaternary radiolarian assemblage, in which species diversity is high (number of species $>100$ ) and specimens are abundant and well preserved, occurs in Core 1 and disappears in Core 2 Section 3. Some of the species represented are (in order of decreasing abundance): Ommatartus tetrathalamus, Pyloniidae group, Dictyocoryne profunda, Euchitonia mulleri, Spongaster tetras, Pterocanium praetexium, P. trilobum, Botryocyrtis spp. and Carpocanistrum spp. There appears to be slight admixture of older Cenozoic sediments.

Sample 2, CC through Core 4, Section 6 are barren.

Sparse and poorly preserved, unidentifiable Radiolaria are present in Sample 4, CC through Core 7, Section 3.

Sample 7, CC contains a poorly preserved but abundant Cretaceous assemblage. The number of specimens declines and their occurrence becomes patchy through Core 11, Section 1.

Sample 11, CC through Core 13, Section 1 contain an abundant, moderate to poorly preserved Cretaceous assemblage quite similar to that found at Site 261, Core 12 , and is therefore assumed to be of the same age (Senonian to Aptian). The same distinctive species are present: Amphipyndax pyrgodes, Dictyomitra lilyae, $D$. pseudoscalaris, D. brouweri, Eucyrtis hanni, E. bulbosus, Lithocampe chenodes, and Eucyrtis columbarius.

From Sample 13, CC to the basalt contact there is a wide fluctuation in abundance, but the preservation remains extremely poor. Very abundant assemblages occur at Core 15, Section 3 to Core 15, Section 4; Core 16, Section 2, 8-10 cm; Core 17, Section 1, 70-72 $\mathrm{cm}$; Core 18, Section 2; and Sample 18, CC. No age determination is possible. c. Site 261 (Argo Abyssal Plain; $12^{\circ} 57^{\prime} \mathrm{S}, 17^{\circ} 54^{\prime} \mathrm{E}$; water depth $5667 \mathrm{~m}$ )

Samples of the top two cores contain a very wellpreserved assemblage of Quaternary radiolarians in which species diversity is high (number of species $>100$ ) and specimens are abundant. Typical species are, in order of decreasing abundance: Ommatartus tetrathalamus, Pyloniidae group, Stylodictya sp. aff. S. multispina, Euchitonia mulleri, Dictyocoryne profunda, Spongaster tetras, Spongotrochus multispinus, Pterocanium praetextum, $P$. trilobum, Lamprocyclas maritalis, Lithopera bacca, Carpocanistrum spp., and Centrobotrys thermophila.

Radiolaria are generally absent in samples from Cores 3 to 8 , although an abundant assemblage appears in Core 8, Sections 3-5. The preservation of these specimens is extremely poor, however, making an age determination impossible.

Core 9 contains a distinct Cretaceous assemblage including Bathropyramis timorensis, Eucyrtidium (?) boodes, and Theocapsa elata. Specimens are poorly preserved.

The material recovered in Core 11 is a chert containing Radiolaria. This was not processed.

Core 12 contains a very abundant and extremely wellpreserved assemblage of Cretaceous (Senonian to Aptian). Radiolaria including the distinctive species Amphipyndax pyrgodes, Dictyomitra lilyae, D. pseudoscalaris, D. brouweri, Eucyrtis hanni, E. bulbosus, E. columbarius, Lithocampe chenodes, and Dictyophimus gracilis, some of which first appear in Core 10. This assemblage continues through Core 23 but adds three new species in Core 15. Abundance fluctuates and preservation declines markedly.

Samples from Cores 24 through 35 (except 25, 26, 33, and 34 , where the material is lithified) generally contain 
TABLE 3

Cretaceous Radiolarians at Site $\mathbf{2 6 0}$

\begin{tabular}{|c|c|c|c|c|c|c|c|c|c|c|c|c|c|c|c|c|c|c|c|c|c|c|c|c|c|c|c|c|c|c|c|c|c|c|c|c|c|c|c|}
\hline SAMPLES & & & | & 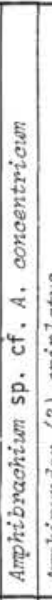 & 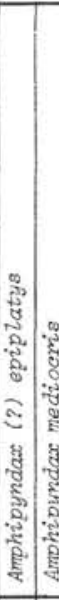 & 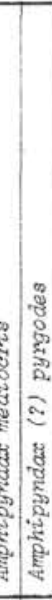 & 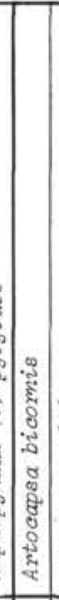 & 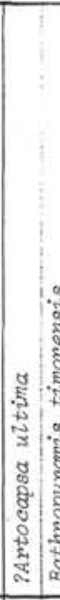 & 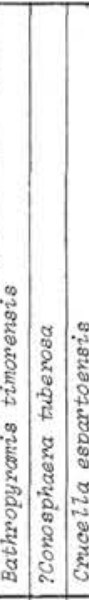 & 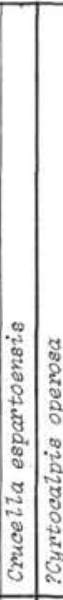 & 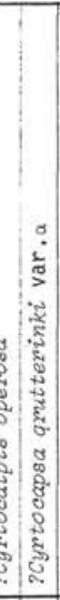 & 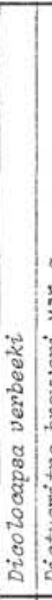 & 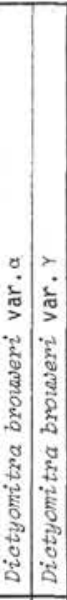 & 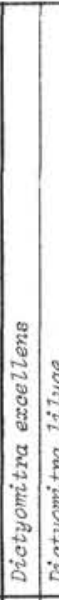 & 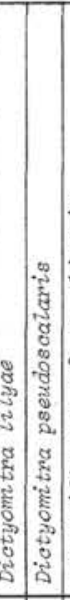 & 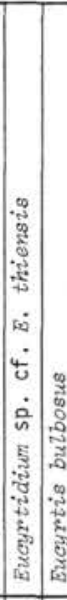 & 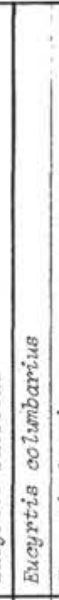 & 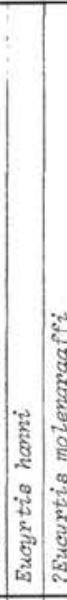 & 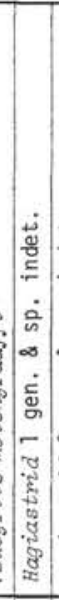 & 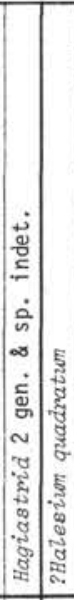 & 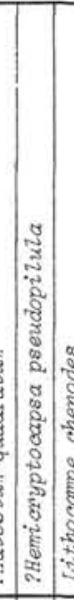 & 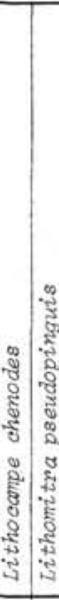 & 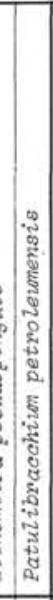 & 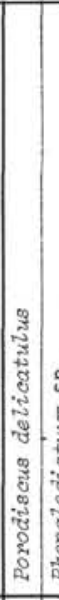 & 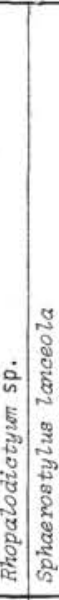 & 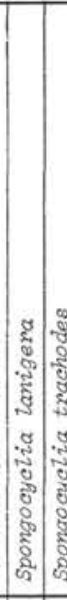 & 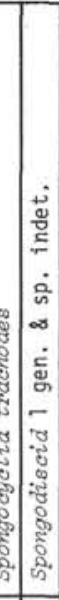 & & & 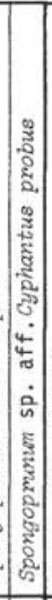 & 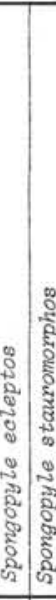 & 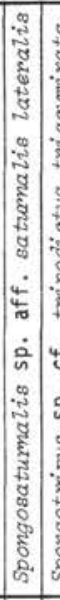 & 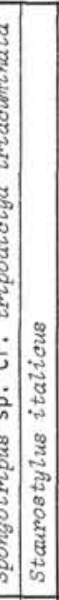 & 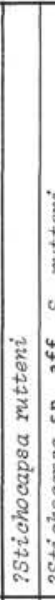 & 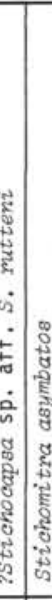 & हु & 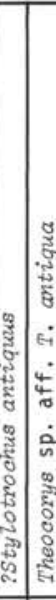 & & 西 \\
\hline $\begin{array}{l}260-9-1,138-140 \\
260-9-c C \\
260-10-1,50-52 \\
260-10-c C \\
260-11-1,113-115\end{array}$ & $\begin{array}{ll}F \\
R \\
F \\
R \\
C\end{array}$ & $\begin{array}{l}M \\
P \\
P \\
M \\
M \\
M\end{array}$ & $R$ & & $\mathrm{~F}$ & -- & & & R & - & $R$ & $F$ & \begin{tabular}{l|l}
$R$ & $F$ \\
- & +
\end{tabular} & $\begin{array}{ll}F & - \\
- \\
+ \\
+ \\
C\end{array}$ & $\begin{array}{l}- \\
-F \\
- \\
- \\
- \\
- \\
- \\
\end{array}$ & - & & $\begin{array}{l}- \\
- \\
-\end{array}$ & - & & $R$ & - & & & & $-1-$ & & & & & & $R$ & & & & & & $R$ & \\
\hline $\begin{array}{l}260-11-C C \\
260-12-1, \quad 131-133 \\
260-12-2,44-46 \\
260-12-2, \quad 130-132 \\
260-12-C C\end{array}$ & $\begin{array}{l}A \\
A \\
F \\
A \\
A\end{array}$ & $\begin{array}{l}P \\
M \\
M \\
M \\
P\end{array}$ & $\begin{array}{l}+ \\
+ \\
F\end{array}$ & + & $\begin{array}{l}+ \\
- \\
- \\
+ \\
+ \\
+ \\
-\end{array}$ & $\begin{array}{ll} & + \\
+ & + \\
+ & + \\
R & \text { A } \\
\end{array}$ & $F$ & F & 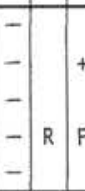 & $+\begin{array}{l}- \\
+ \\
F \\
R\end{array}$ & \begin{tabular}{l|l|l|l} 
& & \\
& & \\
$R$ & $F$ \\
\end{tabular} & $\begin{array}{l}+ \\
+ \\
+\end{array}$ & \begin{tabular}{l|l} 
& + \\
+ \\
+ \\
+ \\
+ \\
+ \\
A & A
\end{tabular} & $\begin{array}{l}- \\
+ \\
- \\
- \\
-\end{array}$ & \begin{tabular}{l|l|}
+ & + \\
+ & + \\
+ & + \\
C & A
\end{tabular} & $\begin{array}{ll}- & - \\
- & - \\
- & - \\
F & F \\
- & F\end{array}$ & $F$ & $\begin{array}{l}-+ \\
+ \\
+ \\
+ \\
A\end{array}$ & $\begin{array}{l}- \\
- \\
- \\
R \\
-\end{array}$ & + & $\begin{array}{lll} & & - \\
+ & - \\
F & F\end{array}$ & \begin{tabular}{l|l}
- & \\
- & \\
& \\
$R$ & $F$
\end{tabular} & {$\left[\begin{array}{l}F \\
+\end{array}\right.$} & c & ++ & \begin{tabular}{|lll}
- & - \\
- & - \\
- & - \\
$R$ & $R$
\end{tabular} & $-\begin{array}{l}+ \\
+ \\
F \\
+\end{array}$ & $R$ & $+\left.\right|_{R} ^{+}$ & $\begin{array}{l}+ \\
+ \\
F\end{array}$ & $R$ & & + & + & \begin{tabular}{l|l}
$R$ & $R$
\end{tabular} & & $\begin{array}{l}+ \\
+ \\
+\end{array}$ & & $F \mid F$ \\
\hline $\begin{array}{l}260-13-1,110-112 \\
260-16-2,8-10 \\
260-17-1,70-72 \\
260-18-2,77.5-79.5 \\
260-18-2,100-102\end{array}$ & $\begin{array}{l}\text { A } \\
\text { A } \\
\text { A }\end{array}$ & & & $F$ & $-R$ & \begin{tabular}{l|l}
$R$ & $R$ \\
\end{tabular} & -1 & R & $\begin{array}{l}+ \\
+\end{array}$ & - & & 7 & $\bar{F}-$ & & & $R$ & + & + & & & & & + & $\begin{array}{l}+ \\
+\end{array}$ & & & & & & & $F$ & & & & & & & & \\
\hline
\end{tabular}


TABLE 4

Quaternary Radiolarians at Site $\mathbf{2 6 0}$

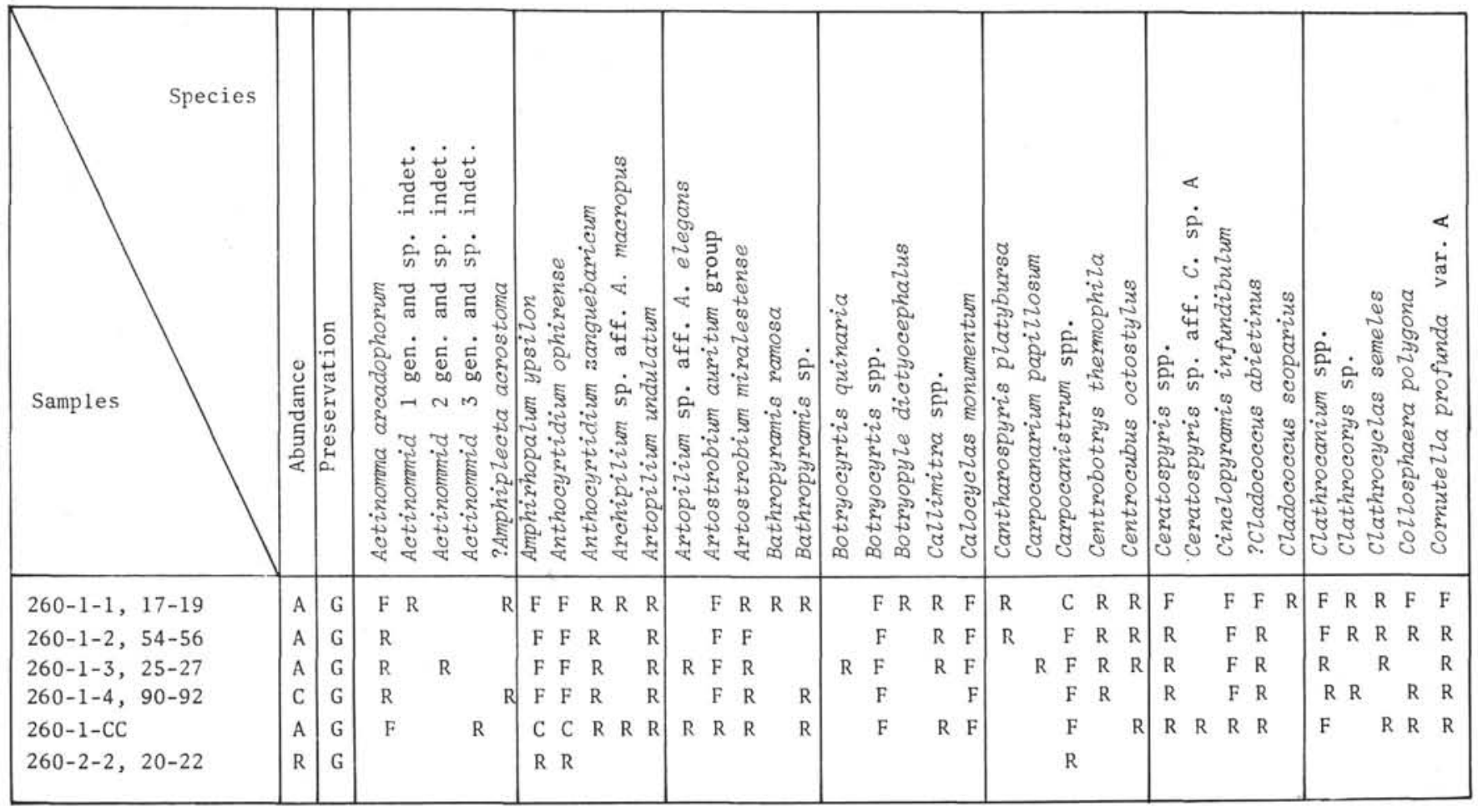

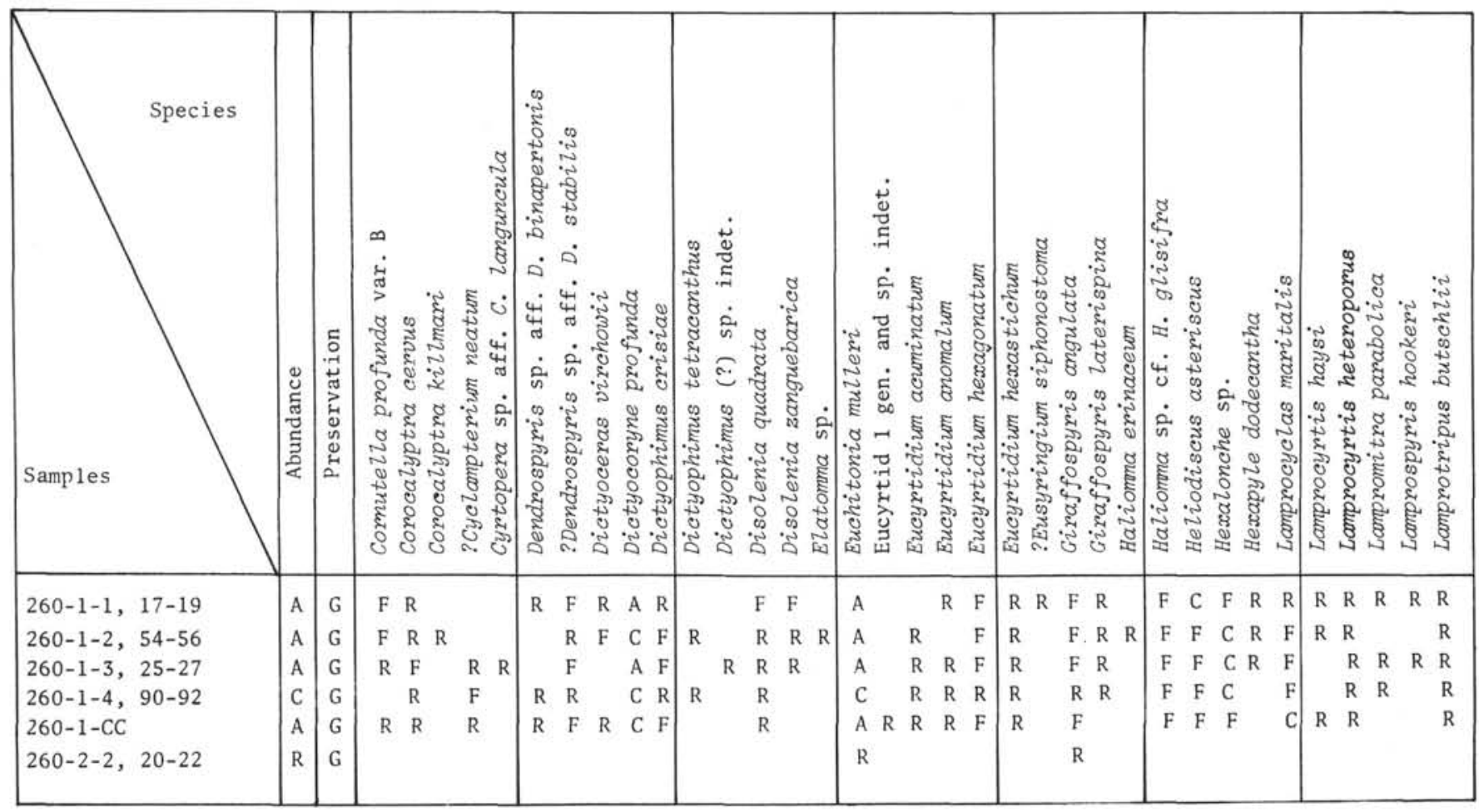


TABLE 4 -Continued

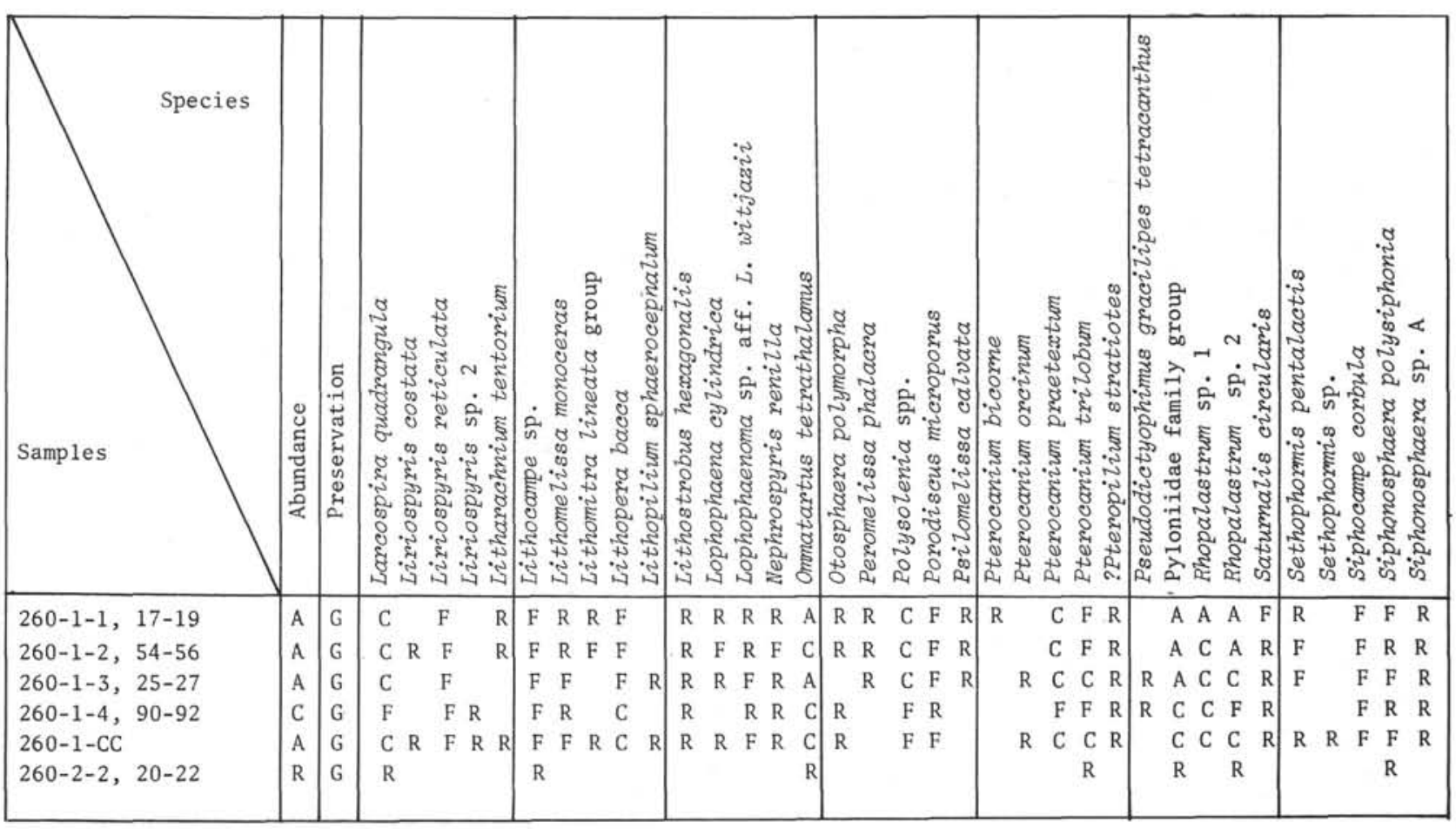

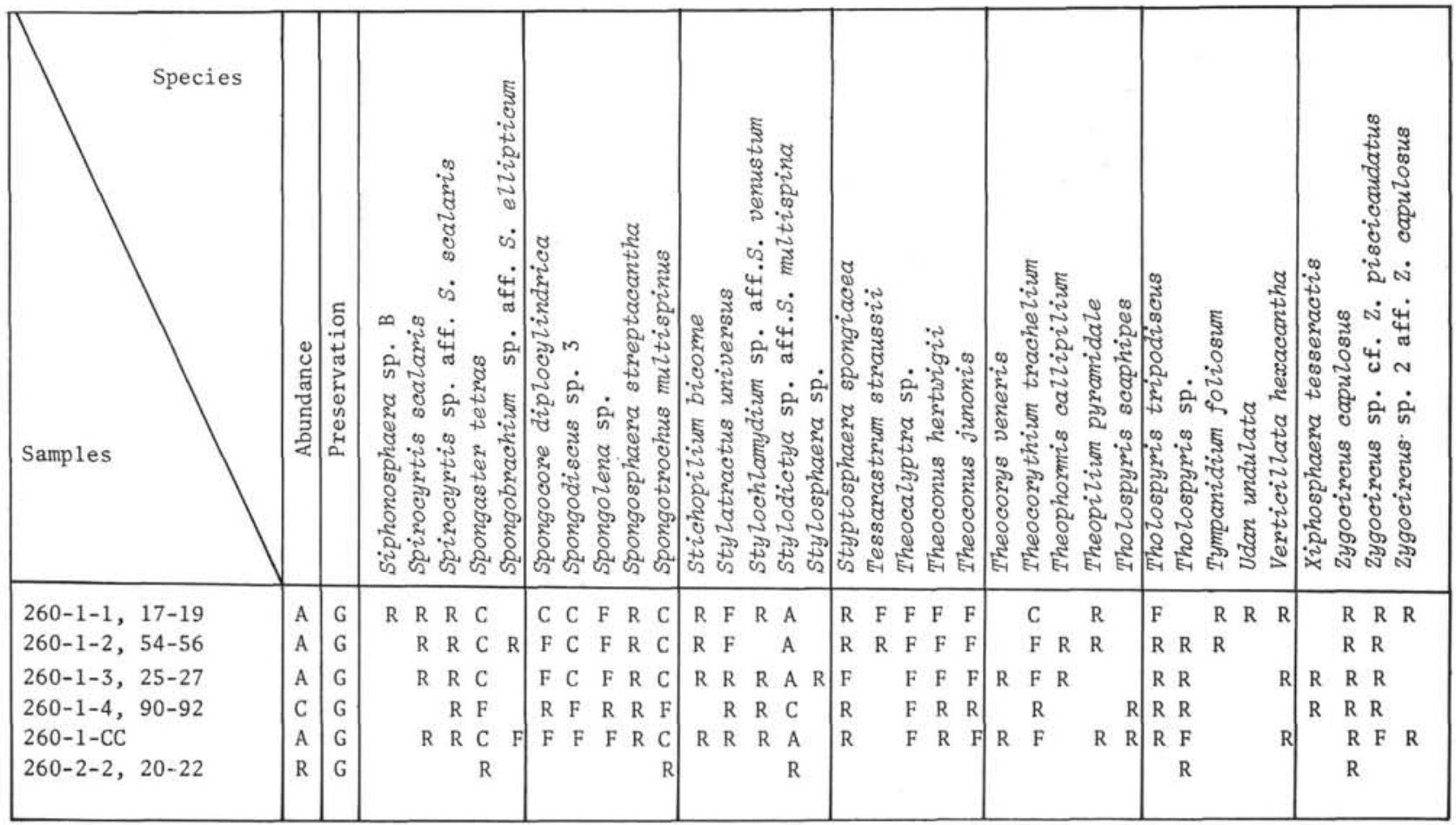


sparse, recrystallized, and badly corroded Radiolaria. Age is indeterminate.

\section{d. Site 262 (Timor Trough; $10^{\circ} 52^{\prime} \mathrm{S}, 1^{\circ} 3^{\circ} 51^{\prime} \mathrm{E}$; water depth $2315 \mathrm{~m}$ )}

Samples from Cores 1 to 25 contain a moderately abundant assemblage of Quaternary radiolarians in which the preservation is good to moderate. Species diversity is high and includes, in order of decreasing abundance: Dictyocoryne profunda, Euchitonia mulleri, Ommatartus tetrathalamus, Pyloniidae group, Pterocanium praetextum, and $P$. trilobum.

Most samples show slight contamination with reworked Cretaceous material including the species Amphipyndax epiplatys and Dictyomitra brouweri.

In samples from Cores 26 to 28 , the same assemblage of Quaternary radiolarians decreases rapidly in abundance and is absent in Core 29. Many specimens are broken.

\section{e. Site 263 (Cuvier Abyssal Plain; $2^{\circ} 20^{\prime} \mathrm{S}, \mathbf{1 1 0}^{\circ} \mathbf{5 8} \mathrm{E}$; water depth $5065 \mathrm{~m}$ )}

Radiolaria are very rare or completely lacking in Site 263 material. Samples from the top three cores contain sparse Quaternary specimens.

Cretaceous radiolarians are present, though patchy and rare, from Core 4 to Core 18. Preservation is extremely poor, making any age determination beyond Cretaceous and any identification beyond radiolarian impossible.

\section{CRETACEOUS STRATIGRAPHY}

The Cretaceous radiolarians in samples recovered from Sites 259, 260, 261, and 263 are, in general, badly corroded, recrystallized, or filled with iron oxides or consolidated sediment. This restricted most tabulations of their occurrence to presence and absence. However, at Site 261 in the interval between Cores 9 and 23 there are three assemblages of particular interest. In this interval radiolarians are most abundant and diverse and show the best preservation in Cores 12 through 16. Since little is known of the stratigraphic sequence of Radiolaria in this part of the geologic column, it seems useful to describe the three assemblages and events (highest and lowest occurrences of species) associated with Site 261 , Cores 9-23. These assemblages are in order from youngest to oldest:

1) Bathropyramis timorensis Assemblage. It is characterized by the presence of Eucyrtidium(?) boodes, Theocapsa elata, and Bathropyramis timorensis and is based on four samples from Core 9. All three species have their lowest occurrence in Sample 9, CC.

2) Eucyrtis columbarius Assemblage. Associated species are Eucyrtis hanni, E. bulbosus, E. columbarius, Lithocampe chenodes, Dictyophimus gracilis, Crucella espartoensis, Spongocyclia trachodes, ?Tripodictya elegantissima, Stylosphaera pusilla, Eucyrtidium vermiculatum with several species of Spongopyle and Dictyomitra. The assemblage has its highest occurrence in Core 12 and its earliest in Core 23. Most of the 10 species mentioned above reach the top of their ranges in Core 12, a few in Core 10 (Core 11 is a chert).
3) Spongocyclia lanigera Assemblage. Characteristic species include Hagiastrid 1 gen. and sp. indet., Lithocyclia (?) sp. A. and Spongocyclia lanigera. These three species reach the top of their range in Core 15 and co-occur with the species of the E. columbarius Assemblage through Core 23.

All three assemblages have these interesting aspects: (1) a complete absence of the family Artostrobiidae, (2) a complete absence of the family Pseudoaulophacidae, (3) a paucity of species in the families Willieriedellidae (1) and Amphipyndacidae (3).

In attempting to assign an age to these assemblages, three different approaches were used in the hope that the results would agree and a firm conclusion could be reached. Unfortunately, this was not the case.

The simplest approach is to relate the age determination given for other microfossils in the same material. Foraminifera from Core 8 have been dated as Senonian (?) or younger and dinoflagellates from Cores 15 to 25 as lower Albian to Aptian.

The second method was to compare stratigraphically important species in the Site 261 sequence with the ranges and zones for these species outlined by Moor (1973) for the northwest Pacific (Leg 17). The comparison was made for Sphaerostylus lanceola, Cyrtocapsa grutterinki $\alpha$, Eucyrtis kruizingai, Stichocapsa procera, Eucyrtis hanni, Stichomitra asymbatos, Dictyomitra brouweri, and Patulibracchium petroleumensis. These species all co-occurred in the Albian-Cenomanian stages.

The last method, although similar to the comparison with Moore's ranges, is farther reaching and more broadly based. Through the work of Riedel and Sanfilippo, six different Cretaceous assemblages were described according to their stratigraphically most important species. These samples are land based (from Western Australia, Spain, and Italy) and marine (from DSDP Legs 11 and 25). Without any prior knowledge of the ages, these assemblages were compared for presence and absence, and, in as objective a manner as possible, arranged in logical stratigraphic order. After the sequence was established, the assemblages from Site 261 were compared and placed at the most appropriate level. When dates were attached to the different samples, Site 261 was bracketed by a Coniacian sample and an Albian sample.

Combining all three methods of age determination, in as conservative a manner as possible, then places Site 261, Cores 9-23, somewhere in the Senonian to Aptian .

Although no age is available, radiolarian assemblages in topotypic material from the Island of Rotti, which neighbors Timor, were studied for comparison. Of the four samples available from the collection of W. R. Riedel, only Rotti Site 149 showed any similarity. Species in common include Dictyomitra brouweri, Amphipyndax mediocris, A. epiplatys, Cornutella californica, Cyrtocalpis operosa, Eucyrtis sp. cf. E. thiensis, Hemicryptocapsa pseudopilula, and Eucyrtis hanni. Several specimens of the Artostrobiidae are present, which, according to method three discussed above, places Site 149, Rotti above (younger) the sequence of Site 261. 
TABLE 5

Cretaceous Radiolarians at Site $\mathbf{2 6 1}$

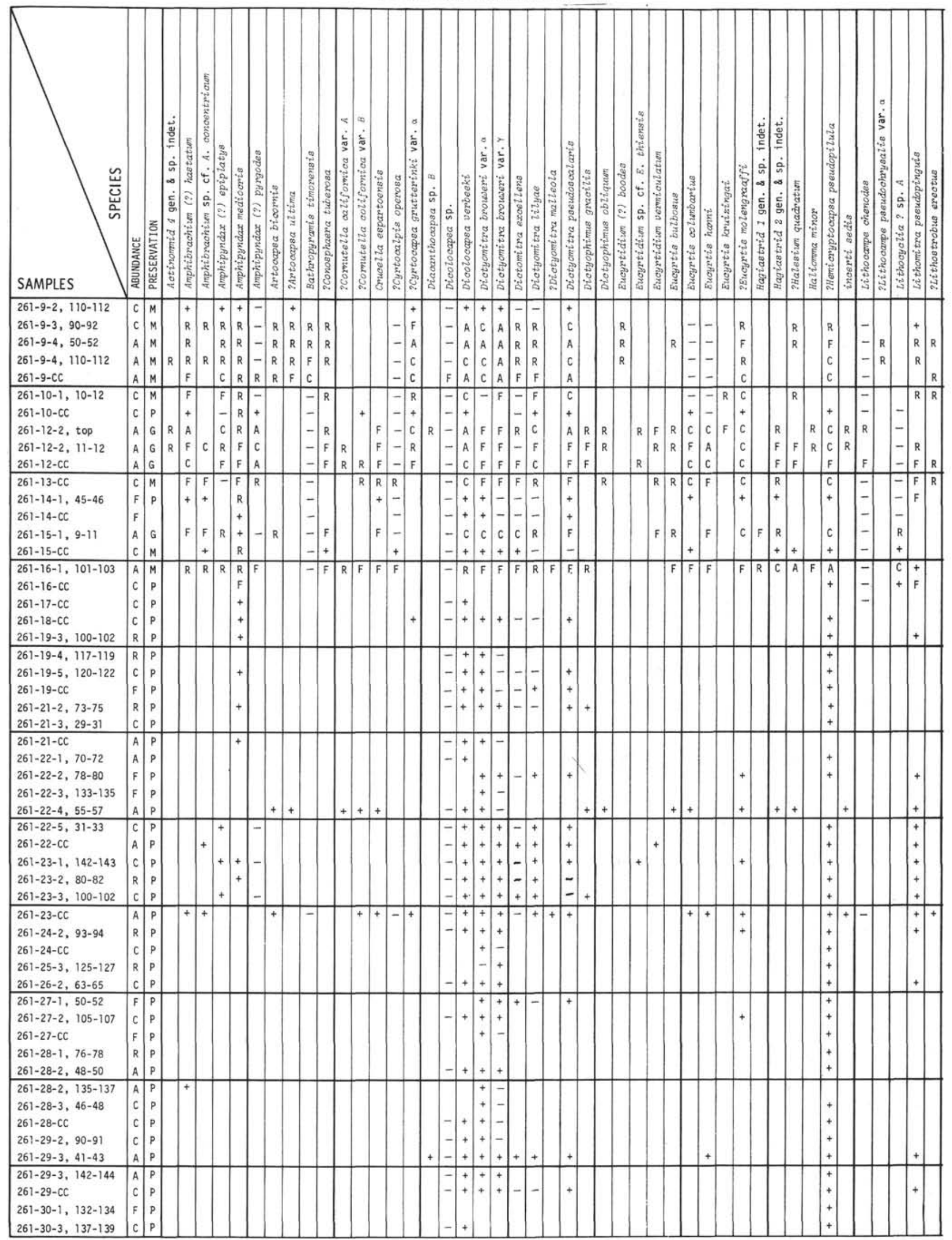


TABLE 5 - Continued

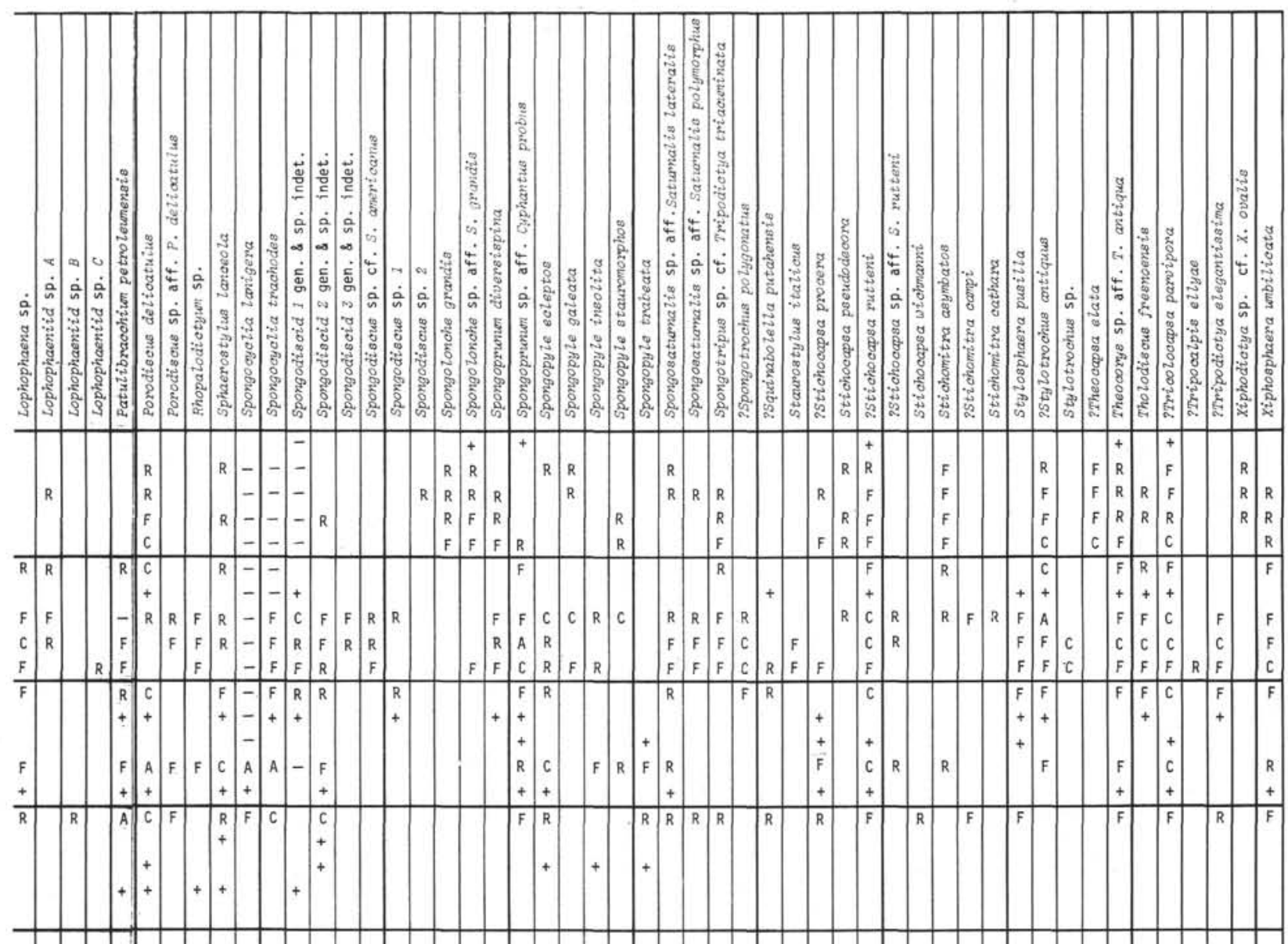


TABLE 6

Quaternary Radiolarians at Site 261

\begin{tabular}{|c|c|c|c|c|c|c|c|c|c|}
\hline Samples & 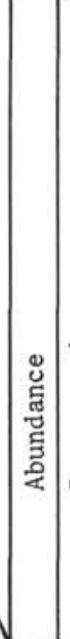 & & 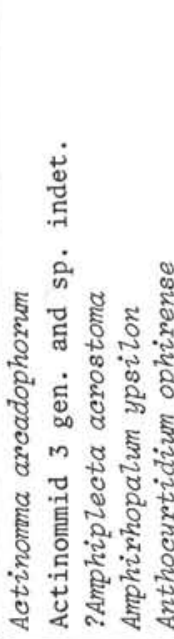 & 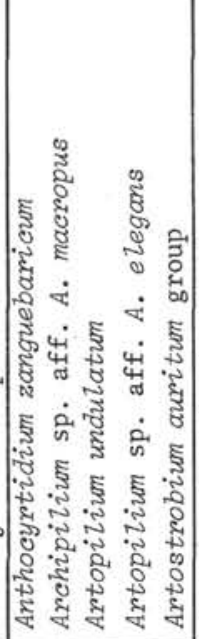 & 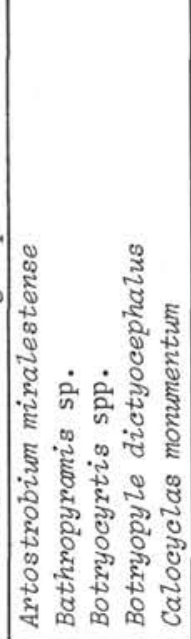 & 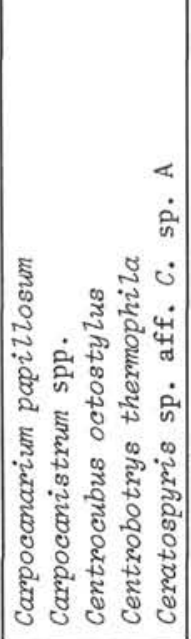 & 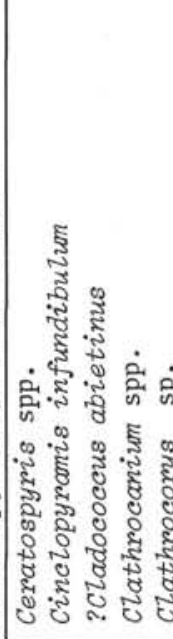 & 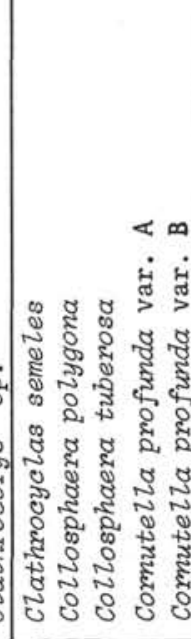 & 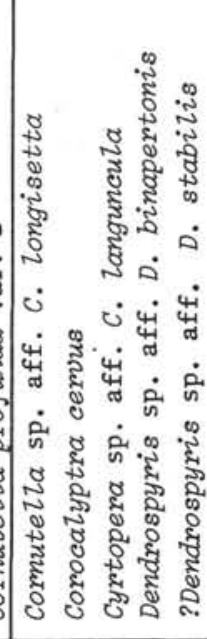 \\
\hline $\begin{array}{l}261-1-C C \\
261-2-C C\end{array}$ & $\begin{array}{l}\text { A } \\
\text { A }\end{array}$ & & $\begin{array}{llll}F & & F & F \\
R & R & R & F\end{array}$ & \begin{tabular}{llll|}
$R$ & $R$ & $F$ \\
$R$ & $R$ & $R$ & $R$
\end{tabular} & $\begin{array}{lllll}R & & F & R \\
F & R & R & R \\
\end{array}$ & \begin{tabular}{|lllll}
$R$ & $F$ & $R$ & $R$ & $R$ \\
$R$ & $C$ & & &
\end{tabular} & $\begin{array}{lllll}F & R & R & F \\
F & R & R & F & R\end{array}$ & $\begin{array}{ll}R & F \\
R & R\end{array}$ & $\begin{array}{lllll} & R & R & R & R \\
& R & & & C\end{array}$ \\
\hline
\end{tabular}

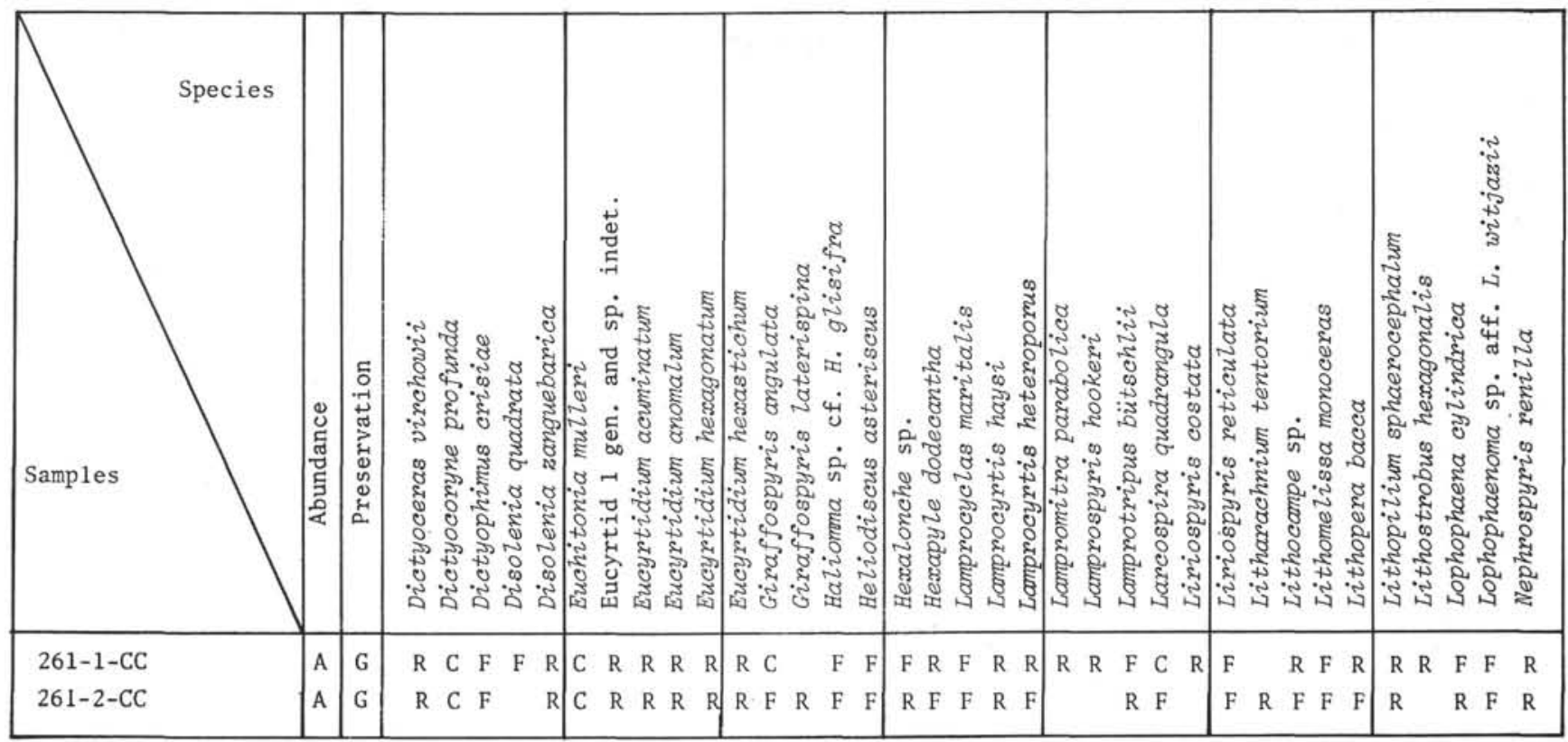


TABLE 6 - Continued

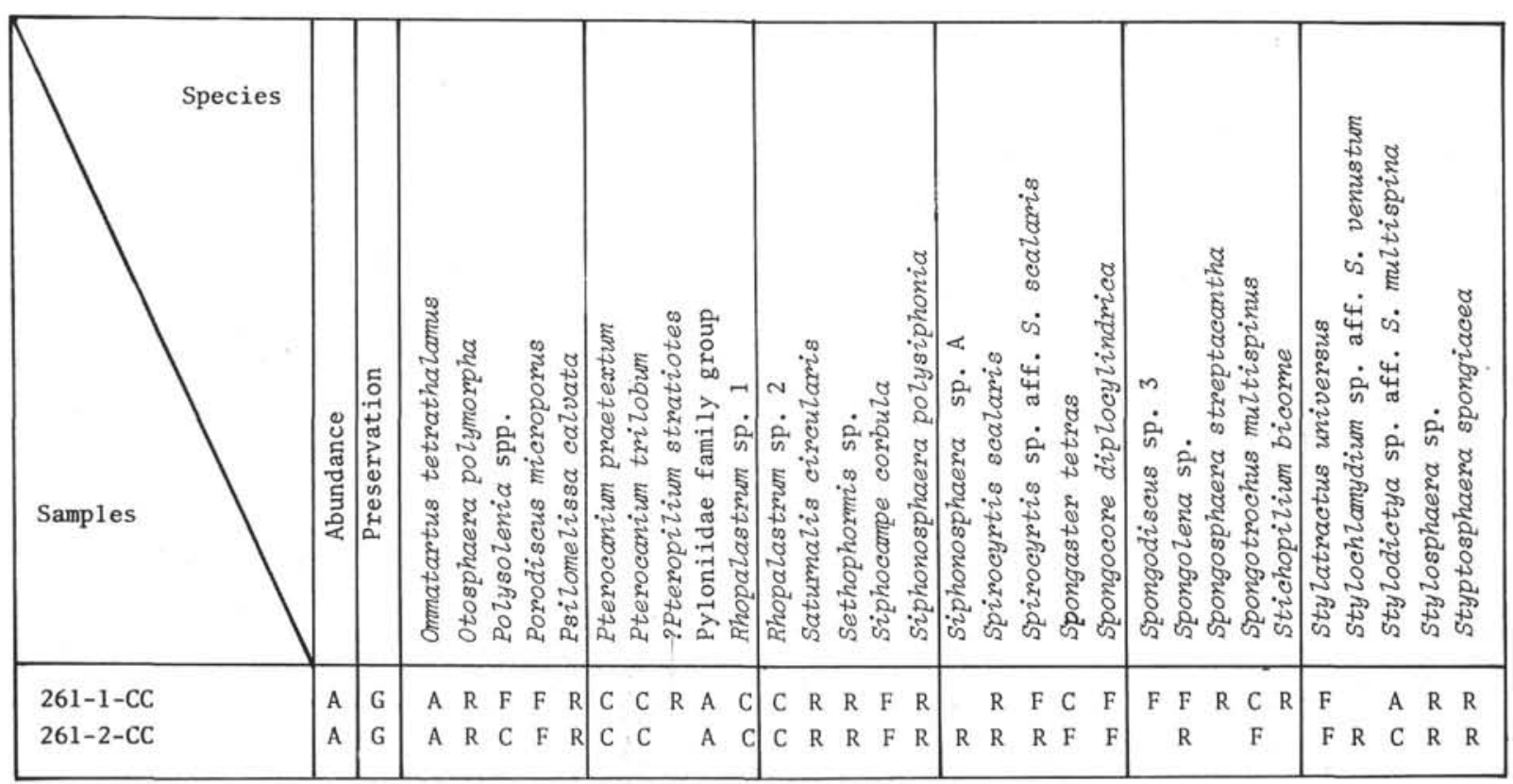

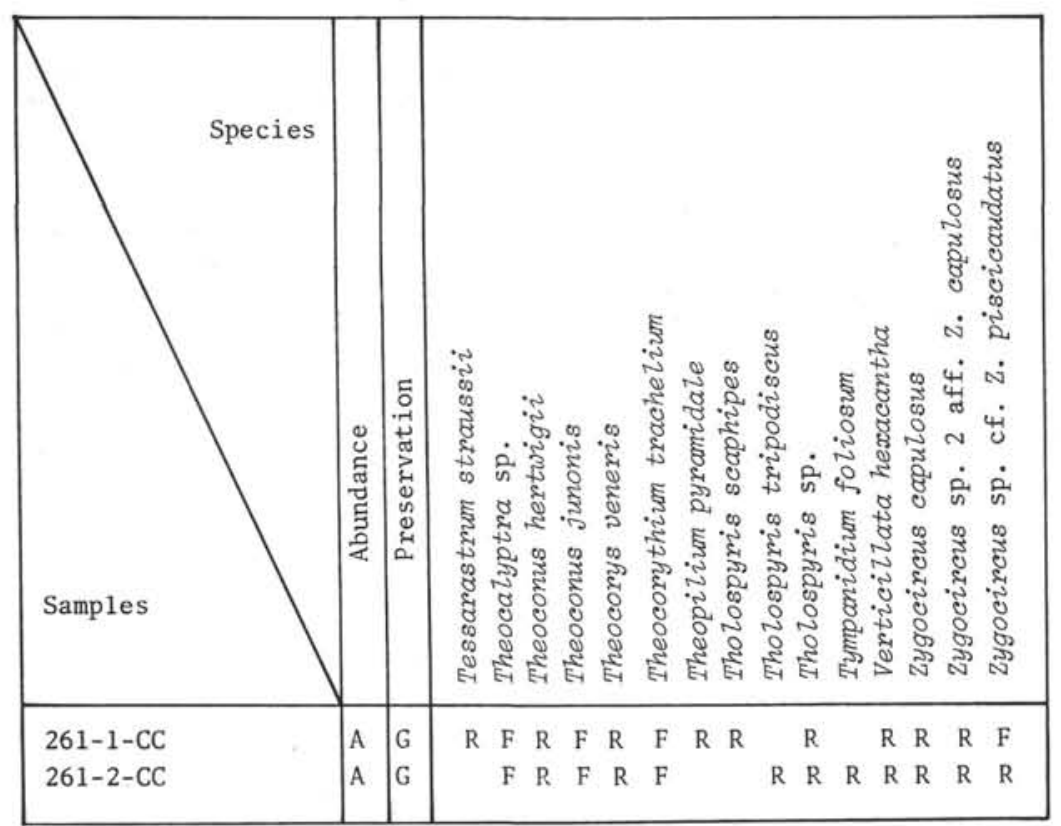


TABLE 7

Quaternary Radiolarians at Site 262

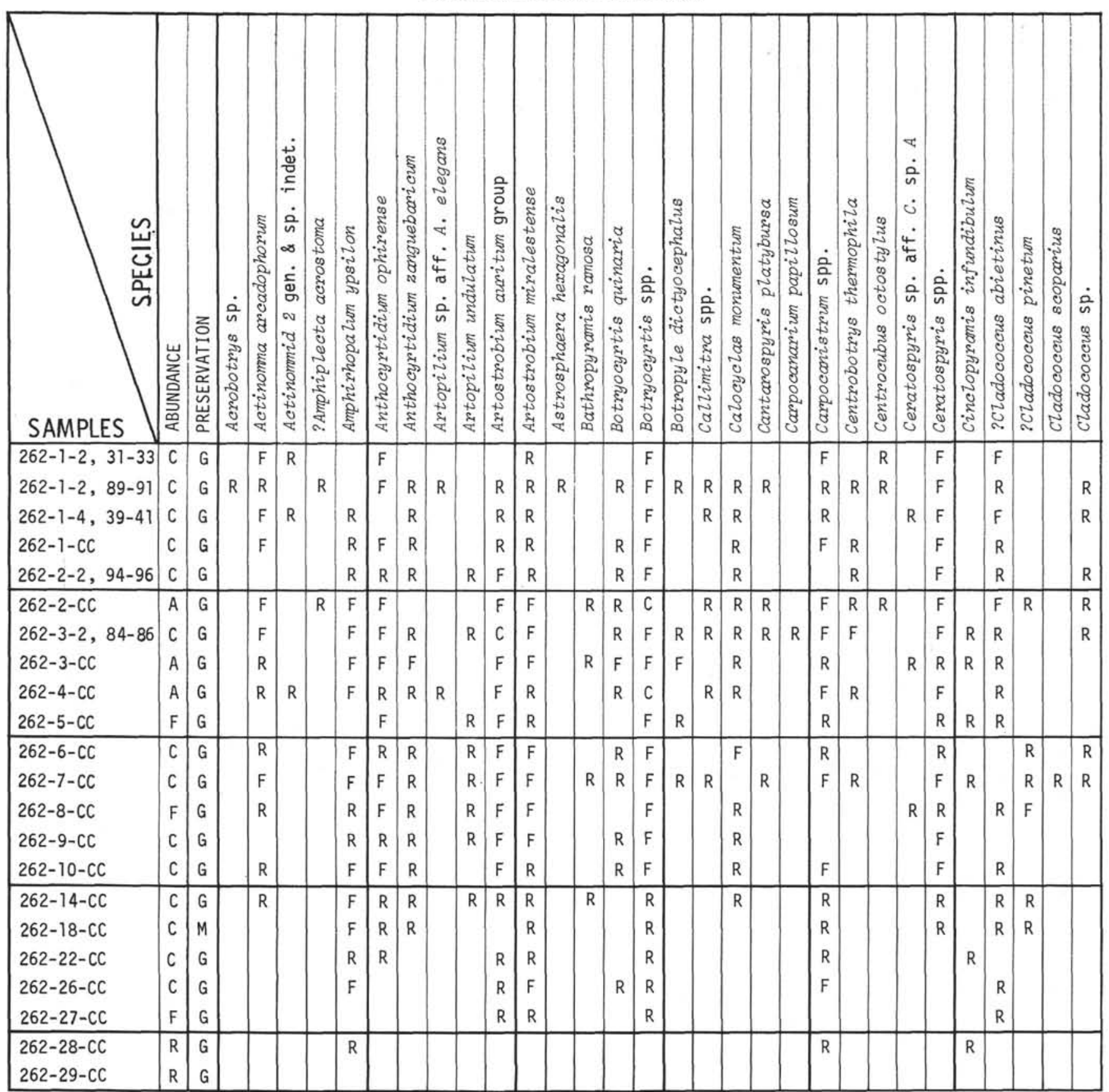




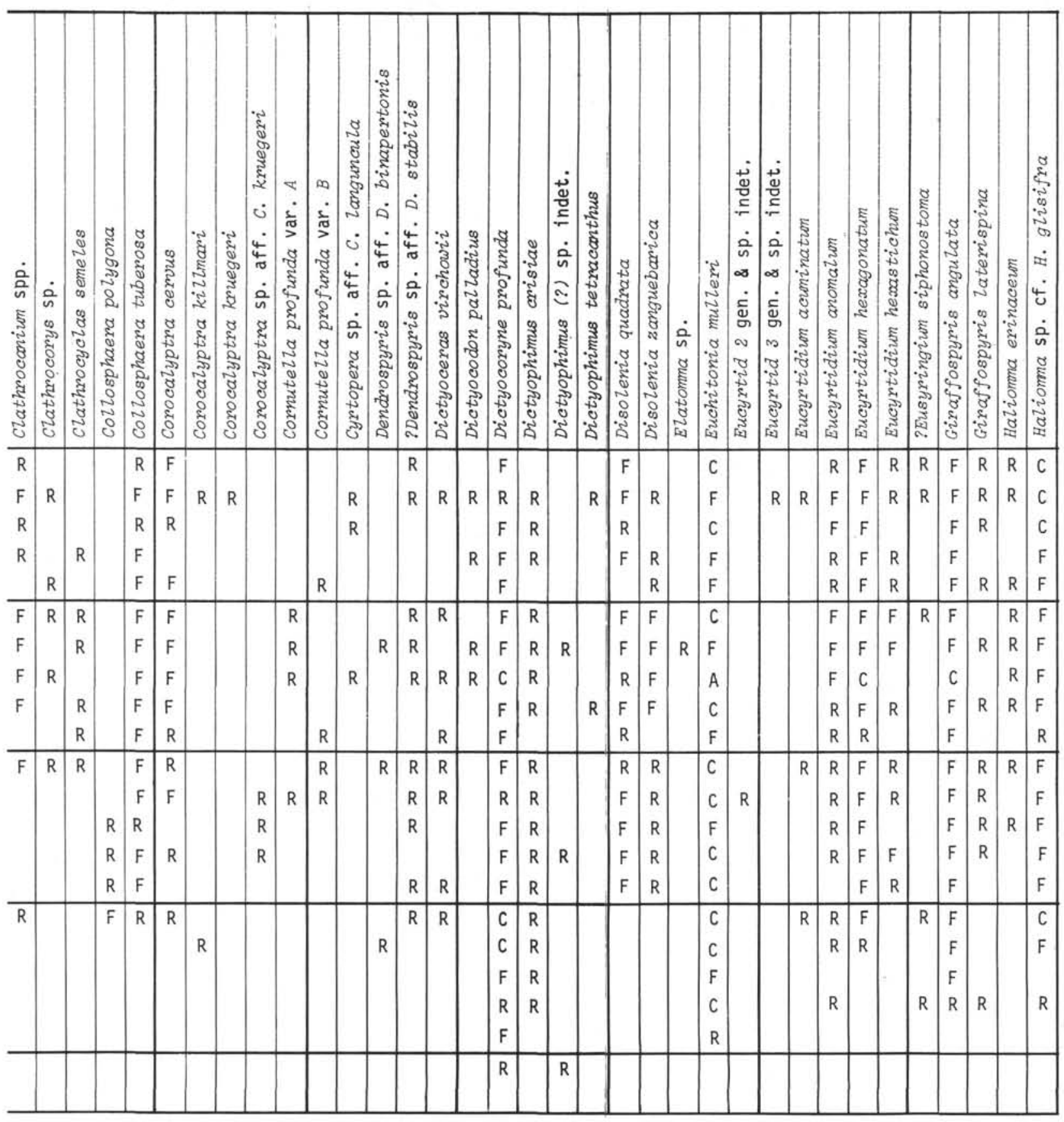


TABLE 7 -Continued

\begin{tabular}{|c|c|c|c|c|c|c|c|c|c|c|c|c|c|c|c|c|c|c|c|c|c|c|c|c|c|c|c|c|c|c|}
\hline $\mid \begin{array}{c}\tilde{m} \\
\bar{w} \\
w \\
\sim \\
\text { SAMPLES }\end{array}$ & 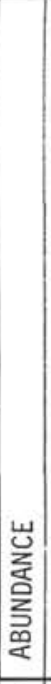 & 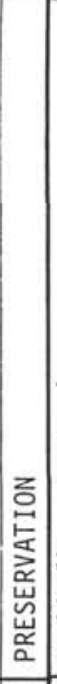 & 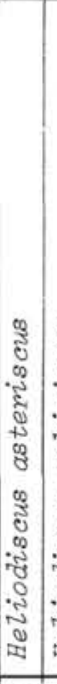 & 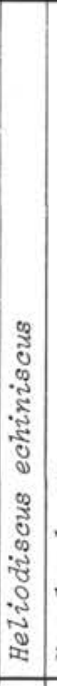 & 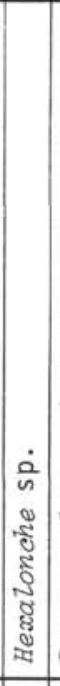 & 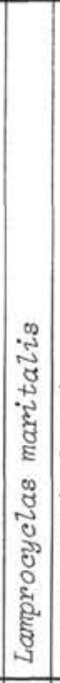 & 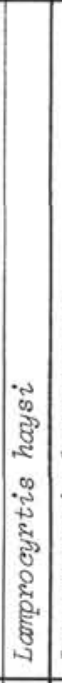 & 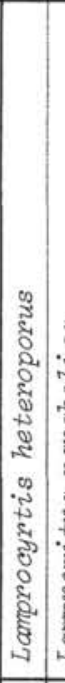 & 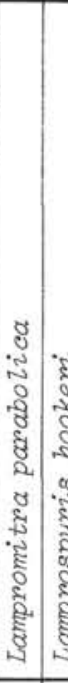 & 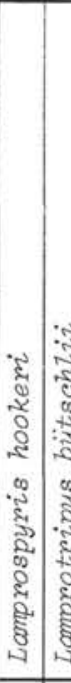 & 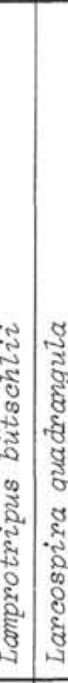 & 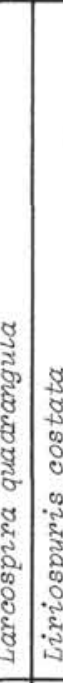 & 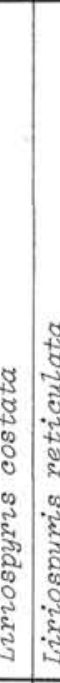 & 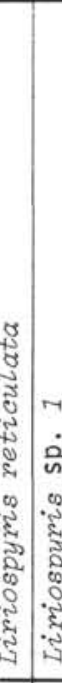 & 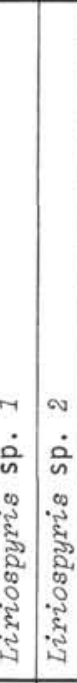 & 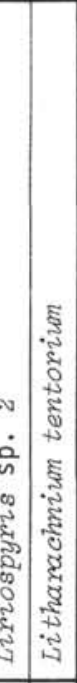 & 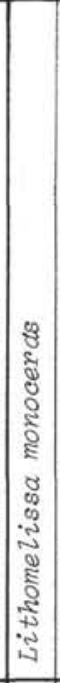 & 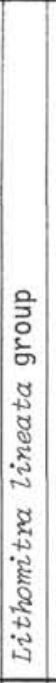 & 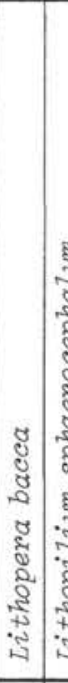 & 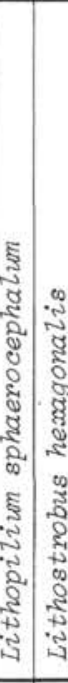 & 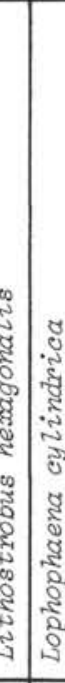 & 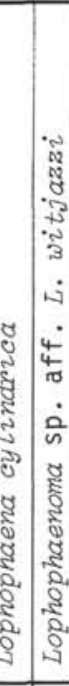 & 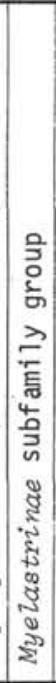 & 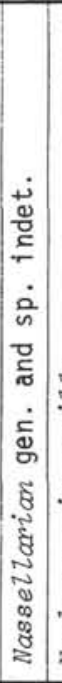 & 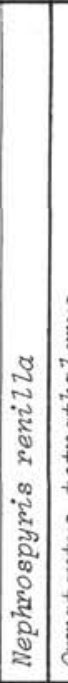 & 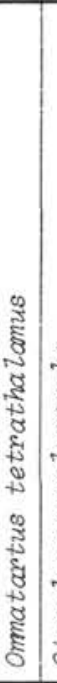 & 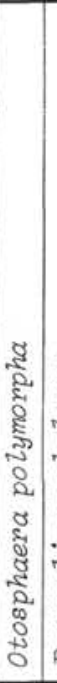 & 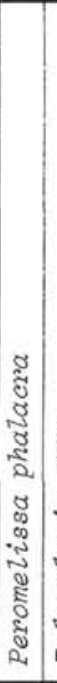 & 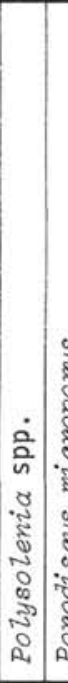 & 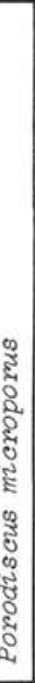 \\
\hline $262-1-2,31-33$ & C & G & $F$ & & & $\mathrm{~F}$ & $\mathrm{~F}$ & & & & & & & $F$ & & & & & & & & $F$ & & & & $\mathrm{R}$ & & & $\mathrm{F}$ & \\
\hline $262-1-2,89-91$ & C & G & $R$ & & & $\mathrm{~F}$ & $R$ & $\mathrm{R}$ & $R \mid F$ & $R \mid F$ & \begin{tabular}{l|l}
$F$ & $R$
\end{tabular} & \begin{tabular}{l|l}
$R$ & $R$
\end{tabular} & \begin{tabular}{l|l}
$R$ & $F$
\end{tabular} & 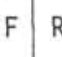 & R & $R$ & $R$ & & $R$ & & \begin{tabular}{l|l}
$R$ & $R$
\end{tabular} & \begin{tabular}{l|l}
$R$ & $F$
\end{tabular} & $\mathrm{R}$ & R & $\mathrm{R}$ & $\mathrm{F}$ & $R$ & $R$ & $\mathrm{~F}$ & \\
\hline $262-1-4,39-41$ & C & G & $\mathrm{R}$ & $R$ & & R & & & & & \begin{tabular}{l|l}
$R$ & $R$
\end{tabular} & R & & & & & $\mathrm{R}$ & & $\mathrm{R}$ & & & $R$ & & $R$ & & $F$ & & $R$ & $\mathrm{~F}$ & $\mathrm{R}$ \\
\hline $262-1-C C$ & C & G & $F$ & & $R$ & $\mathrm{~F}$ & & & & & $\mathrm{~F} \quad \mathrm{~F}$ & $F$ & \begin{tabular}{l|l}
$R$ & $R$
\end{tabular} & $R$ & \begin{tabular}{l|l}
$R$ & $R$
\end{tabular} & $R|R|$ & $R$ & & $R$ & & & \begin{tabular}{l|l}
$R$ & $F$
\end{tabular} & & & $R$ & C & $\mathrm{R}$ & $\mathrm{R}$ & $\mathrm{R}$ & \\
\hline $262-2-2,94-96$ & $\mathrm{C}$ & G & $\mathrm{R}$ & & $\mathrm{F}$ & $\mathrm{F}$ & R & & & & \begin{tabular}{l|l}
$R$ & $F$ \\
\end{tabular} & \begin{tabular}{l|l}
$F$ & $R$ \\
\end{tabular} & \begin{tabular}{l|l}
$R$ & $F$ \\
\end{tabular} & $\mathrm{~F}$ & & & & & $\mathrm{R}$ & & & \begin{tabular}{l|l}
$R$ & $F$ \\
\end{tabular} & & & $R$ & $\mathrm{~F}$ & & & $\mathrm{~F}$ & $\mathrm{R}$ \\
\hline $262-2-C C$ & A & G & $\mathrm{F}$ & $R$ & $F$ & $F$ & $R$ & & & & \begin{tabular}{l|l}
$R$ & $F$
\end{tabular} & $F$ & & \begin{tabular}{l|l}
$F$ & $R$ \\
\end{tabular} & \begin{tabular}{l|l}
$R$ & $R$ \\
\end{tabular} & \begin{tabular}{|l|l|}
$R$ & $R$ \\
\end{tabular} & $F$ & & $R$ & & & \begin{tabular}{l|l}
$F$ & $C$
\end{tabular} & & & $F$ & C & $F$ & $\mathrm{R}$ & $\mathrm{F}$ & $F$ \\
\hline $262-3-2,84-86$ & C & G & $\mathrm{F}$ & & $\mathrm{F}$ & C & $R$ & & $\mathrm{R} / \mathrm{F}$ & $\mathrm{R} \mid \mathrm{F}$ & $\mathrm{F} F$ & 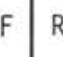 & \begin{tabular}{l|l}
$\mathrm{R}$ & $\mathrm{F}$
\end{tabular} & 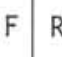 & R & $\mathrm{F}$ & $\mathrm{F}$ & $\mathrm{R}$ & $R$ & & \begin{tabular}{l|l}
$\mathrm{R}$ & $\mathrm{F}$
\end{tabular} & $F$ & $\mathrm{R}$ & & $\mathrm{R}$ & C & $\mathrm{F}$ & $\mathrm{F}$ & $\mathrm{F} F$ & $\mathrm{R}$ \\
\hline $262-3-C C$ & A & G & R & & $\mathrm{F}$ & F & $\mathrm{F}$ & $\mathrm{F}$ & & & \begin{tabular}{l|l}
$\mathrm{R}$ & $\mathrm{C}$
\end{tabular} & $C \mid \mathrm{R}$ & \begin{tabular}{l|l}
$R$ & $F$
\end{tabular} & $\mathrm{~F}$ & & $\mathrm{~F}$ & $\mathrm{R}$ & & $\mathrm{R}$ & & & $F$ & & & $F$ & c & $\mathrm{F}$ & $\mathrm{R}$ & $\mathrm{R} F$ & $\mathrm{R}$ \\
\hline $262-4-C C$ & A & G & F & $R$ & $\mathrm{~F}$ & $\mathrm{~F}$ & $\mathrm{R}$ & $R$ & $R \mid F$ & $R$ & \begin{tabular}{l|l}
$\mathrm{R}$ & $\mathrm{C}$
\end{tabular} & \begin{tabular}{l|l}
$C$ & $R$
\end{tabular} & \begin{tabular}{l|l}
$R$ & $F$
\end{tabular} & $\mathrm{~F}$ & & & $\mathrm{R}$ & & $R$ & & $R \mid R$ & \begin{tabular}{l|l}
$R$ & $F$
\end{tabular} & & & $R$ & A & $\mathrm{F}$ & & $F$ & $R$ \\
\hline $262-5-C C$ & $\mathrm{~F}$ & G & $\mathrm{F}$ & & $\mathrm{F}$ & $\mathrm{F}$ & & & & & \begin{tabular}{l|l}
$R$ & $F$
\end{tabular} & $F$ & & $\mathrm{R}$ & & & $\mathrm{R}$ & & & & \begin{tabular}{l|l}
$R$ & $R$ \\
\end{tabular} & \begin{tabular}{l|l}
$R$ & $R$
\end{tabular} & & & $R$ & $\mathrm{~F}$ & $R$ & $R$ & $\mathrm{~F}$ & $\mathrm{R}$ \\
\hline $262-6-C C$ & C & $G$ & $\mathrm{~F}$ & & & $F$ & $R$ & & \begin{tabular}{l|l}
$R$ & $R$
\end{tabular} & \begin{tabular}{l|l}
$R$ & $F$
\end{tabular} & \begin{tabular}{l|l}
$F$ & $C$
\end{tabular} & C & \begin{tabular}{l|l}
$R$ & $R$ \\
\end{tabular} & $R$ & & $R$ & $R$ & & $R$ & & \begin{tabular}{l|l}
$R$ & $F$
\end{tabular} & \begin{tabular}{l|l}
$F$ & $F$ \\
\end{tabular} & & & $R$ & C & $\mathrm{R}$ & $\mathrm{R}$ & $\mathrm{F}$ & $R$ \\
\hline $262-7-C C$ & C & G & F & & F & $\mathrm{F}$ & $\mathrm{F}$ & $R$ & $R$ & & \begin{tabular}{l|l}
$R$ & $C$
\end{tabular} & c & & $F$ & $R$ & $R$ & $\mathrm{~F}$ & & R $F$ & \begin{tabular}{l|l}
$R$ & $R$
\end{tabular} & \begin{tabular}{l|l}
$R$ & $F$
\end{tabular} & \begin{tabular}{l|l}
$F$ & $F$
\end{tabular} & & & $R$ & C & & & $\mathrm{F}$ & $R$ \\
\hline $262-8-C C$ & F & G & $R$ & & F & $\mathrm{F}$ & & & & & & $F$ & \begin{tabular}{l|l}
$\mathrm{R}$ & $\mathrm{R}$
\end{tabular} & $R$ & & & $\mathrm{R}$ & & $R$ & $R$ & & \begin{tabular}{l|l}
$R$ & $R$
\end{tabular} & & & $R$ & $\mathrm{~F}$ & & & $\mathrm{R}$ & \\
\hline $262-9-C C$ & C & G & $\mathrm{F}$ & & F & $\mathrm{F}$ & & F & & & \begin{tabular}{l|l}
$R$ & $C$
\end{tabular} & C & & $\mathrm{F}$ & R & $R$ & $\mathrm{R}$ & & $\mathrm{R}$ & & \begin{tabular}{l|l}
$\mathrm{R}$ & $\mathrm{F}$
\end{tabular} & $\mathrm{F}$ & & & $R$ & C & $\mathrm{R}$ & & $\mathrm{F}$ & $R$ \\
\hline $262-10-C C$ & $C$ & G & $\mathrm{F}$ & & $\mathrm{F}$ & $\mathrm{F}$ & & $\mathrm{R}$ & & & \begin{tabular}{l|l}
$R$ & $C$
\end{tabular} & C & & $\mathrm{F}$ & $\mathrm{R}$ & R & $\mathrm{R}$ & & $\mathrm{R}$ & & \begin{tabular}{l|l}
$R$ & $F$
\end{tabular} & $\mathrm{~F}$ & & & $R$ & C & $\mathrm{R}$ & & $\mathrm{F}$ & $\mathrm{R}$ \\
\hline $262-14-C C$ & C & G & $\mathrm{F}$ & & $\mathrm{F}$ & $R$ & $R$ & $\mathrm{R}$ & & & \begin{tabular}{l|l}
$F$ & $F$ \\
\end{tabular} & $F$ & & $R$ & & & & & $R$ & & \begin{tabular}{l|l}
$R$ & $R$ \\
\end{tabular} & \begin{tabular}{l|l}
$R$ & $R$ \\
\end{tabular} & & & $R$ & $\mathrm{~F}$ & & & \begin{tabular}{l|l}
$C$ & $F$
\end{tabular} & $F$ \\
\hline $262-18-C C$ & C & M & $\mathrm{F}$ & & $\mathrm{F}$ & $\mathrm{F}$ & $\mathrm{R}$ & $\mathrm{F}$ & & & $R$ R & $\mathrm{F}$ & & & & & & & & & & $\mathrm{R}$ & & & & $\mathrm{F}$ & $\mathrm{R}$ & & $\mathrm{F}$ & $R$ \\
\hline $262-22-C C$ & C & G & $R$ & & $\mathrm{~F}$ & $R$ & & $R$ & & & & $\mathrm{~F}$ & & & & & & & & & & & & & & $\mathrm{~F}$ & & & $\mathrm{~F}$ & $\mathrm{R}$ \\
\hline $262-26-C C$ & C & G & $\mathrm{F}$ & & $\mathrm{F}$ & $\mathrm{F}$ & & & & & & $R$ & & $\mathrm{R}$ & & & & & $\mathrm{R}$ & & & $R \mid R$ & & & & C & & & $\mathrm{F}$ & \\
\hline $262-27-C C$ & $\mathrm{~F}$ & G & $R$ & & $\mathrm{~F}$ & $R$ & $R$ & & & & & $R$ & & & & & & & $R$ & & & & & & & $\mathrm{~F}$ & & & $\mathrm{R}$ & $\mathrm{R}$ \\
\hline $262-28-C C$ & $R$ & $\bar{G}$ & $R$ & & & $R$ & & & & & & & & & & & & & & & & & & & & $R$ & & & & $R$ \\
\hline $262-29-C C$ & $R$ & G & & & & & & & & & & & & & & & & & & & & & & & & & & & $R$ & \\
\hline
\end{tabular}


TABLE 7 - Continued

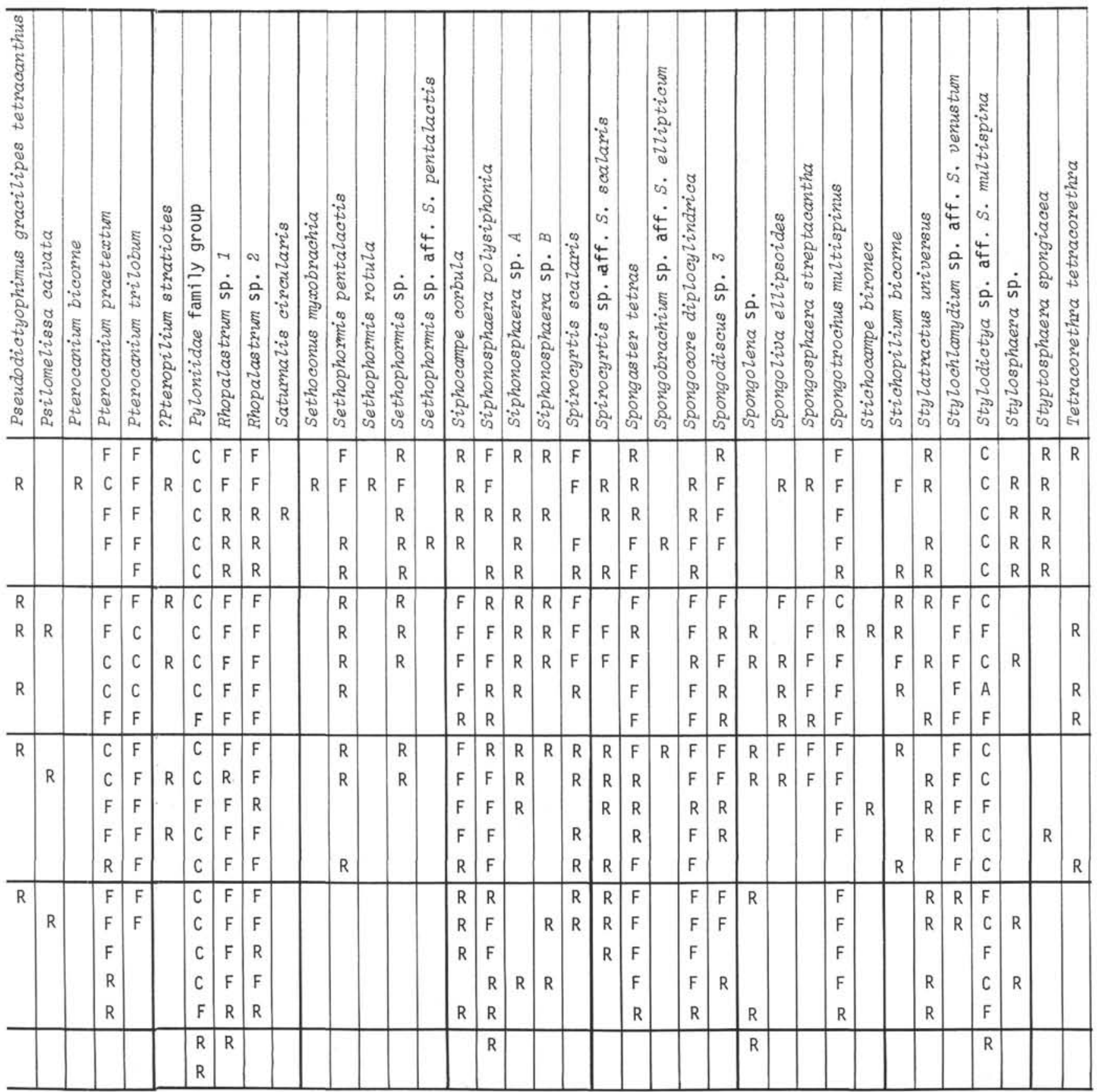


TABLE 7 -Continued

\begin{tabular}{|c|c|c|c|c|c|c|c|c|c|c|c|c|c|c|c|c|c|c|}
\hline $\mid \begin{array}{c}\tilde{w} \\
\bar{u} \\
\frac{w}{\sim} \\
\text { SAMPLES }\end{array}$ & 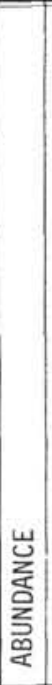 & 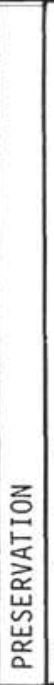 & 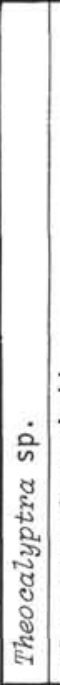 & 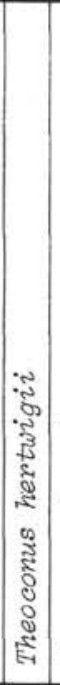 & 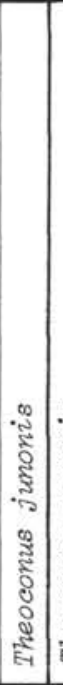 & 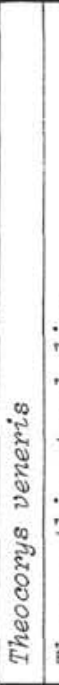 & 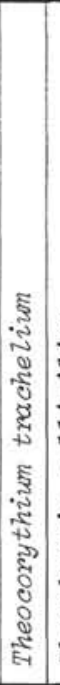 & 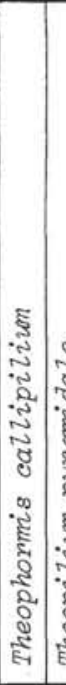 & 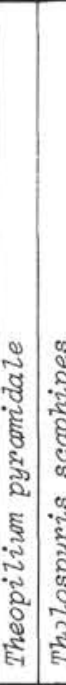 & 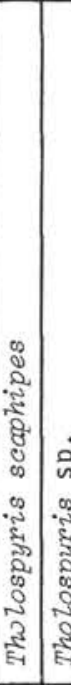 & 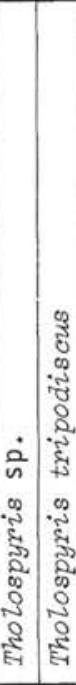 & 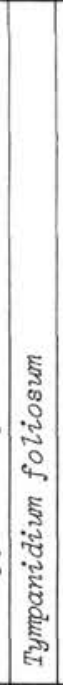 & 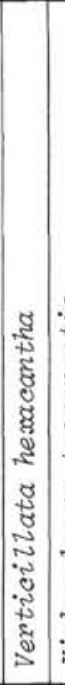 & 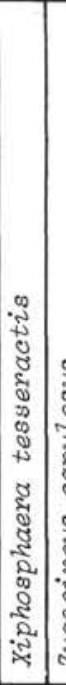 & 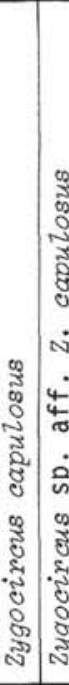 & 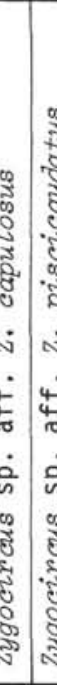 & 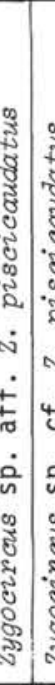 & 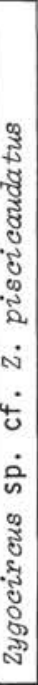 \\
\hline $262-1-2,31-33$ & C & $G$ & $F$ & $\mathrm{~F}$ & $\mathrm{C}$ & & & & & $R$ & & $R$ & $R$ & & \begin{tabular}{l|l}
$\mathrm{F}$ & $\mathrm{R}$
\end{tabular} & $\bar{R}$ & $R$ & $r$ \\
\hline $262-1-2,89-91$ & C & G & c & $F$ & C & $R$ & $\mathrm{R}$ & & $\mathrm{F} F$ & $R \mid R$ & \begin{tabular}{l|l}
$R$ & $R$
\end{tabular} & $\mathrm{~F}$ & $\mathrm{R}$ & & $\mathrm{F}$ & & & $r$ \\
\hline $262-1-4,39-41$ & C & G & $\mathrm{F}$ & R & $\mathrm{F}$ & $\mathrm{R}$ & & & & $\mathrm{R}$ & & $\mathrm{R}$ & & & $\mathrm{F}$ & & & $\mathrm{F}$ \\
\hline $262-1-C C$ & c & G & $\mathrm{F}$ & & $\mathrm{F}$ & $R$ & $R$ & & $R$ & & & $\mathrm{R}$ & & & $\mathrm{F}$ & & & \\
\hline $262-2-2,94-96$ & C & G & $R$ & $R$ & $\mathrm{~F}$ & & $R$ & & & \begin{tabular}{l|l}
$R$ & $R$ \\
\end{tabular} & $R$ & & $R$ & & $\mathrm{R}$ & & & \\
\hline $262-2-C C$ & $A$ & G & $\mathrm{F}$ & $F$ & C & & $\mathrm{F}$ & & \begin{tabular}{l|l}
$R$ & $R$
\end{tabular} & $R$ & & & & & $F$ & & & $F$ \\
\hline $262-3-2,84-86$ & $c$ & G & $\mathrm{F}$ & $\mathrm{F}$ & $\mathrm{F}$ & $\mathrm{R}$ & $\mathrm{F}$ & & F $P$ & R & $\mathrm{R}$ & & R & & $\mathrm{F} \mid \mathrm{R}$ & R & & \\
\hline $262-3-C C$ & $A$ & G & $F$ & $\mathrm{R}$ & C & $R$ & $R$ & & $\mathrm{~F} \quad \mathrm{P}$ & $R$ & R & & $\mathrm{F}$ & & c & & & \\
\hline $262-4-C C$ & A & G & $\mathrm{F}$ & $\mathrm{R}$ & $\mathrm{F}$ & $\mathrm{R}$ & $\mathrm{R}$ & & & $R \mid R$ & R & & $\mathrm{R}$ & & $\mathrm{F} F$ & R & & R \\
\hline $262-5-C C$ & $\mathrm{~F}$ & G & $\mathrm{F}$ & & $\mathrm{F}$ & & & $R$ & & $\mathrm{R}$ & & $\mathrm{R}$ & $R$ & & $\mathrm{R}$ & & & $R$ \\
\hline $262-6-C C$ & C & G & $R$ & $R$ & $F$ & & & & $R$ & & & $\mathrm{R}$ & $R$ & & \begin{tabular}{l|l}
$\mathrm{F}$ & $\mathrm{F}$
\end{tabular} & $R$ & & $R$ \\
\hline $262-7-C C$ & C & G & $\mathrm{F}$ & R & F & $\mathrm{R}$ & $R$ & $\mathrm{R}$ & R & & R & F & & & $\mathrm{R} F$ & $\mathrm{R}$ & R & \\
\hline $262-8-C C$ & F & G & $R$ & & F & & $R$ & & & & R & & & $R$ & & $R$ & & $R$ \\
\hline $262-9-C C$ & C & G & $\mathrm{F}$ & & $F$ & & $R$ & & & & & & & & $\mathrm{R}$ & & & $R$ \\
\hline $262-10-C C$ & C & G & $\mathrm{R}$ & & C & & R & & $R \quad$ & $\mathrm{R}$ & & & & & $\mathrm{R}$ & & & $\mathrm{R}$ \\
\hline $262-14-C C$ & $C$ & G & $R$ & $R$ & $F$ & & $R$ & $R$ & & \begin{tabular}{l|l}
$R$ & $R$ \\
\end{tabular} & $R$ & $R$ & & & \begin{tabular}{l|l}
$R$ & $F$
\end{tabular} & $R$ & & \\
\hline $262-18-C C$ & c & M & $R$ & $\mathrm{R}$ & $\mathrm{F}$ & & $R$ & & & & & & & $\mathrm{R}$ & & & & \\
\hline $262-22-C C$ & c & G & & & $\mathrm{R}$ & $R$ & & & & & & & & & $R$ & & & $R$ \\
\hline $262-26-C C$ & C & G & & & $R$ & & $R$ & & & & & & & $R$ & & & & $R$ \\
\hline $262-27-C C$ & $\mathrm{~F}$ & G & & & & & & & & & & & & & & & & $\mathrm{F}$ \\
\hline $262-28-C C$ & $R$ & $G$ & & & & & & & & & & & & & & & & 8 \\
\hline $262-29-C C$ & $\mathrm{R}$ & G & & & & & & & & & & & & & & & & \\
\hline
\end{tabular}


TABLE 8

Quaternary Radiolarians at Site $\mathbf{2 6 3}$

\begin{tabular}{|c|c|c|c|c|c|c|c|c|c|c|c|c|c|c|c|c|}
\hline Samples & 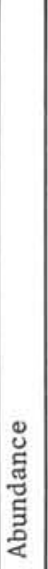 & 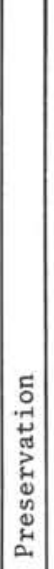 & 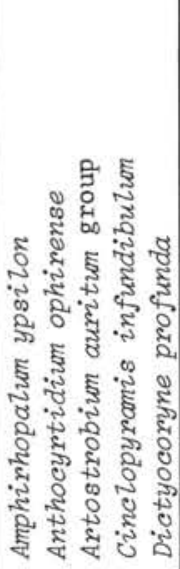 & 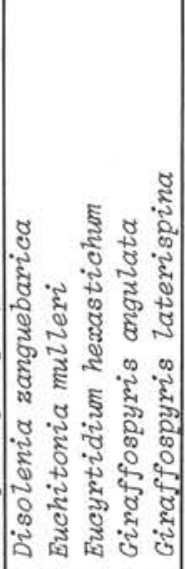 & 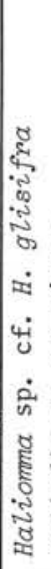 & 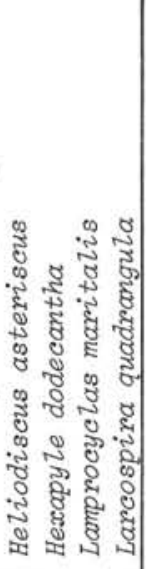 & 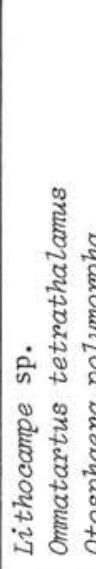 & 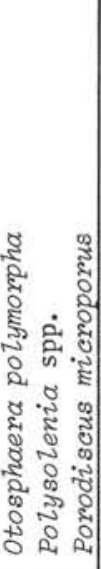 & 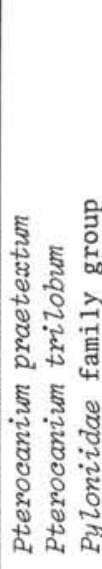 & 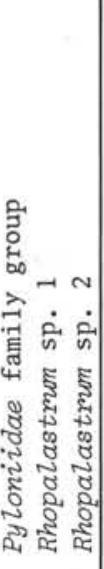 & 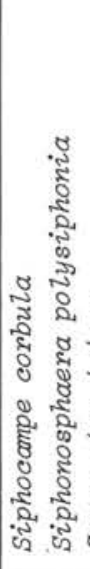 & 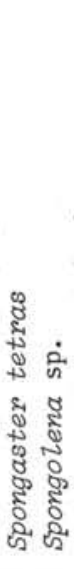 & 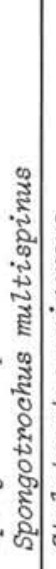 & 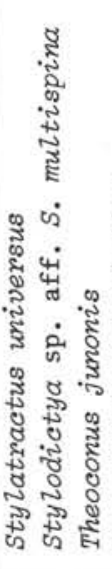 & 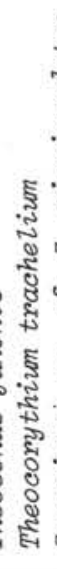 & 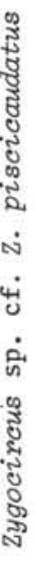 \\
\hline $\begin{array}{l}263-1-1,110-112 \\
263-1-2,92-94 \\
263-1-C C \\
263-2-4,46-48 \\
263-2-C C\end{array}$ & $\begin{array}{l}\mathrm{R} \\
\mathrm{R} \\
\mathrm{R} \\
\mathrm{F} \\
\mathrm{R}\end{array}$ & $\begin{array}{l}\mathrm{G} \\
\mathrm{G} \\
\mathrm{G} \\
\mathrm{M} \\
\mathrm{M}\end{array}$ & $\begin{array}{lll}R & R & \\
R & & R \\
& & R\end{array}$ & $\begin{array}{llll}\mathrm{R} & & \mathrm{R} \\
& & & \\
& \mathrm{R} & \mathrm{R} & \end{array}$ & $\mathrm{R}$ & \begin{tabular}{lll|l}
$R$ & & $R$ & \\
$R$ & & $R$ & \\
$R$ & $R$ & & $R$
\end{tabular} & ${ }_{R}^{R}$ & $\begin{array}{lll}R & R \\
& R & R\end{array}$ & $\mathrm{R} R$ & $\begin{array}{lll}R & R \\
F & R & R \\
R & \end{array}$ & $\mathrm{R}$ & $\mathrm{R}$ & $\mathrm{R}$ & $\begin{array}{lll}R & R & \\
& R & \\
& R & R \\
& R & \\
& R & R\end{array}$ & $\begin{array}{l}R \\
R \\
R \\
R\end{array}$ & $\begin{array}{l}R \\
R \\
R\end{array}$ \\
\hline
\end{tabular}

After having studied the sequence of Cores 12-16, Site 261 , the other poorly preserved cores above and below it, along with cores from other sites, were examined in an attempt to recognize any assemblage similarities. The Bathropyramis timorensis Assemblage was defined in Core 9, and the Eucyrtis columbarius and Spongocyclia lanigera assemblages were followed and last recognized in Core 23. In other sites, the E. columbarius Assemblage alone was found at Site 260, Core 12.

\section{SYSTEMATICS}

Following is an outline of species classification. The precedures listed next were used in this presentation:

1) Spumellaria were subdivided according to the suprageneric classification of Riedel (1971) with the exception that the Porodiscidae were split from the Spongodiscidae (Petrushevskaya and Kozlova, 1972) and the Hagiastridae were elevated to the family level (Pessagno, 1971).

2) Nassellaria were subdivided according to the suprageneric classification of Petrushevskaya (1971a).

3) Specific identification followed Haeckel (1887), except for new species described by later investigators.

Following the outline is an alphabetical listing of individual species encountered in this study. The reference following the plate and figure number in this chapter designates the concept of the species adopted by the author. This may not necessarily conform completely with the original description. Locations of reference to each species are noted after "This chapter" by special designations for each of the topics within the chapter. This replaces the traditional index. The final notation of Quaternary or Cretaceous refers to the age of the material in which the species was found.

Fifteen new species are introduced. Type specimens will be deposited in the United States National Museum.

\section{a. Classification Outline}

Subclass Radiolaria

Superorder Polycystina Ehrenberg, 1838, emend. Riedel, 1967a

Order Spumellaria Ehrenberg, 1875

Family Collosphaeridae Muller 1858, emend. Riedel, 1971

Collosphaera polygona Haeckel, 1887

Collosphaera tuberosa Haeckel, 1887
Disolenia quadrata (Ehrenberg, 1872a)

Disolenia zanguebarica (Ehrenberg, 1872a)

Otosphaera polymorpha $\mathrm{Haeckel,} 1887$

Polysolenia spp.

Siphonosphaera polysiphonia Haeckel, 1887

Siphonosphaera sp. A

Siphonosphaera sp. B

Family Litheliidae Haeckel, 1862

Larcospira quadrangula Haeckel, 1887

Family Phacodiscidae Haeckel, 1881

Heliodiscus asteriscus Haeckel, 1887

Heliodiscus echiniscus Haeckel, 1887

Family Pylodiscidae Haeckel, 1887

Hexapyle dodecantha Haeckel, 1887

Family Pyloniidae Haeckel, 1881

Pyloniidae family group

Family Liosphaeridae Haeckel, 1881

Styptosphaera spongiacea Haeckel 1887

Family Actinommidae Haeckel, 1862, emend. Riedel, 1971

Actinomma arcadophrum Haeckel, 1887

Actinommid 1 gen. and sp. indet.

Actinommid 2 gen. and sp. indet.

Actinommid 3 gen. and sp. indet.

Actinommid 4 gen. and sp. indet.

Astrosphaera hexagonalis Haeckel, 1887

Centrocubus octostylus Haeckel, 1887

?Cladococcus abietinus Haeckel, 1887

?Cladococcus pinetum Haeckel, 1887

Cladococcus scoparius Haeckel, 1887

Cladococcus sp.

?Conosphaera tuberosa Tan Sin Hok, 1927

Elatomma sp.

Haliomma erinaceum Haeckel, 1862

Haliomma minor Campbell and Clark, 1944b

Haliomma sp. aff, H. glisifra Renz, in press

Hexalonche $\mathrm{sp}$.

Sphaerostylus lanceola (Parona, 1890)

Spongosphaera streptacantha Haeckel, 1862

Staurostylus italicus Rüst, 1898

Stylatractus universus Hays, 1970

Stylosphaera pusilla Campbell and Clark, 1944b

Stylosphaera sp.

Xiphosphaera tesseractis Dreyer, 1913

Xiphosphaera umbilicata Rust, 1898 
Subfamily Saturnalinae Deflandre, 1953

Saturnalis circularis Haeckel, 1887

Spongosaturnalis sp. aff. Saturnalis lateralis group Campbell and Clark, 1944b

Spongosaturnalis sp. aff. Saturnalis polymorphus Squinabol, 1914

Subfamily Artiscinae Haeckel, 1881, emend. Riedel, 1971

Ommatartus tetrathalamus (Haeckel, 1887)

Spongoliva ellipsoides Popofsky, 1912

Family Porodiscidae Haeckel, 1881, emend. Petrushevskaya and Kozlova, 1972

Amphirhopalum ypsilon Haeckel, 1887

Porodiscus delicatulus (Lipman, 1952)

Porodiscus microporous (Stöhr, 1880)

Porodiscus sp. aff. P. delicatulus (Lipman, 1952)

Stylodictya sp. aff. S. multispina Haeckel, 1862

Stylochlamydium sp. aff. $S$. venustum (Bailey, 1856)

Tessarastrum straussii Haeckel, 1887

Tholodiscus fresnoensis (Foreman, 1968)

Tripodictya elegantissima Vinassa, 1899

Xiphodictya sp. cf. X. ovalis Rust, 1898

Family Hagiastridae Riedel, 1971, emend. Pessagno, 1971

Amphibrachium (?) hastatum n. sp.

Crucella espartoensis Pessagno, 1971

Hagiastrid 1 gen. and sp. indet.

Hagiastrid 2 gen. and sp. indet.

?Halesium quadratum Pessagno, 1971

Patulibracchium petroleumensis Pessagno, 1971

Family Spongodiscidae Haeckel, 1862, emend. Petrushevskaya and Kozlova, 1972

Amphibrachium sp. cf. A. concentricum Lipman, 1960

Dictyocoryne profunda Ehrenberg, 1872b

Euchitonia mulleri Haeckel, 1862

Lithocyclia? sp. A

Rhopalastrum sp. 1

Rhopalastrum sp. 2

Rhopalodictyum $\mathrm{sp.}$

Spongaster tetras Ehrenberg, 1860

Spongobrachium sp. aff. S. ellipticum Haeckel, 1862

Spongocore diplocylindrica $\mathrm{Haeckel,} 1887$

Spongocyclia lanigera $\mathrm{n} . \mathrm{sp}$.

Spongocyclia trachodes $\mathrm{n}$. $\mathrm{sp}$.

Spongodiscid 1 gen. and sp. indet.

Spongodiscid 2 gen. and sp. indet.

Spongodiscid 3 gen. and sp. indet.

Spongodiscus sp. cf. S. americanus Kozlova and Gorbovets, 1966

Spongodiscus sp. 1

Spongodiscus sp. 2

Spongodiscus sp. 3

Spongolena sp.

?Spongolonche grandis Campbell and Clark, 1944b

Spongolonche sp. aff. S. grandis Campbell and Clark, 1944b

Spongoprumum diversispina Squinabol, 1904

Spongoprunum sp. aff. Cyphantus probus Rüst, 1888

Spongopyle ecleptos $\mathrm{n}$. sp.

Spongopyle galeata $\mathrm{n} . \mathrm{sp}$.

Spongopyle insolita Kozlova and Gorbovets, 1966

Spongopyle stauromorphos $\mathrm{n}$. $\mathrm{sp}$.

Spongopyle trabeata $\mathrm{n} . \mathrm{sp}$.

Spongotripus sp. cf. Tripodictya triacuminata Lipman, 1952

Spongotrochus multispinus Haeckel, 1887

?Spongotrochus polygonatus Campbell and Clark, 1944b

?Stylotrochus antiquus Campbell and Clark, 1944a

Stylotrochus sp.

Subfamily Myelastrinae Riedel, 1971

Myelastrinae subfamily group

Order Nassellaria Ehrenberg, 1875

Nassellarian gen. and sp. indet.

Suborder Cyrtida Haeckel, 1862, emend. Petrushevskaya, 1971a

Superfamily Eucyrtidioidea Ehrenberg, 1847, emend.

Petrushevskaya, 1971a

Family Eucyrtidiidae Ehrenberg, 1847, emend.

Petrushevskaya, 1971a
Archipilium sp. aff. A. macropus (Haeckel, 1887)

Artocapsa bicornis Tan Sin Hok, 1927

?Artocapsa ultima Tan Sin Hok, 1927

Artopilium undulatum Popofsky, 1913

Artopilium sp. aff. A. elegans Haeckel, 1887

Calocyclas monumentum Haeckel, 1887

Clathrocyclas semeles Haeckel, 1887

Coroclayptra cervus (Ehrenberg, 1872b)

Corocalyptra killmari Renz, in press

Corocalyptra kruegeri Popofsky, 1908

Corocalyptra sp. aff. C. kruegeri Popofsky, 1908

?Cyclampterium neatum Sanfilippo and Riedel, 1970

?Cyrtocapsa grutterinki var. $\alpha$ Tan Sin Hok, 1927

Cyrtopera sp. aff. C. languncula Haeckel, 1887

Diacanthocapsa sp. B

Dicolocapsa verbeeki Tan Sin Hok, 1927

Dicolocapsa sp.

Dictyoceras virchowii Haeckel, 1862

Dictyocodon palladius Haeckel, 1887

Dictyomitra brouweri var. $\alpha$ (Tan Sin Hok, 1927)

Dictyomitra brouweri var. $\gamma$ (Tan Sin Hok, 1927)

Dictyomitra excellens (Tan Sin Hok, 1927)

Dictyomitra lilyae (Tan Sin Hok, 1927)

?Dictyomitra malleola Aliev, 1961

Dictyomitra pseudoscalaris (Tan Sin Hok, 1927)

Dictyophimus crisiae Ehrenberg, 1854

Dictyophimus gracilis Tan Sin Hok, 1927

Dictyophimus obliquum (Hinde, 1900)

Dictyophimus(?) sp. indet.

Eucyrtid 1 gen. and sp. indet.

Eucyrtid 2 gen. and sp. indet.

Eucyrtid 3 gen. and sp. indet.

Eucyrtidium acuminatum (Ehrenberg, 1844)

Eucyrtidium anomatum Haeckel, 1862

Eucyrtidium (?) boodes n. sp.

Eucyrtidium hexastichum (Haeckel, 1887)

Eucyrtidium hexagonatum Haeckel, 1887

Eucyrtidium vermiculatum $\mathrm{n}$. sp.

Eucyrtidium sp. cf. E. thiensis Tan Sin Hok, 1927

Eucvrtis bulbosus n. sp.

Eucyrtis columbarius $\mathrm{n}$. sp.

Eucyrtis hanni (Tan Sin Hok, 1927)

Eucyrtis kruizingai (Tan Sin Hok, 1927)

?Eucyrtis molengraaffi (Tan Sin Hok, 1927)

?Eusyringium siphonostoma Haeckel, 1887

Lampromitra parabolica Popofsky, 1913

Lithocampe chenodes $\mathrm{n}$. $\mathrm{sp}$.

?Lithocampe pseudochrysalis var. $\alpha$ Tan Sin Hok, 1927

Lithocampe sp.

Lithomitra pseudopinguis Tan Sin Hok, 1927

Lithopera bacca Ehrenberg, 1872a

Lithopilium sphaerocephalum Popofsky, 1913

Lithostrobus erectus Tan Sin Hok, 1927

Lithostrobus hexagonalis Haeckel, 1887

Pterocanium bicorne Haeckel, 1887

Pterocanium orcinum Haeckel, 1887

Pterocanium praetextum (Ehrenberg, 1872a)

Pterocanium trilobum (Haeckel, 1860)

Sethoconus myxobrachia Strelkov and Reshetnyak, 1959

?Squinabolella putahensis Pessagno, 1969

Stichocampe bironec Renz, in press

?Stichocapsa p:ocera Hinde, 1908

Stichocapsa pseudodecora Tan Sin Hok, 1927

?Stichocapsa sp. aff. S. rutteni Tan Sin Hok, 1927

?Stichocapsa rutteni Tan Sin Hok, 1927

Stichocapsa wichmanni Tan Sin Hok, 1927

Stichomitra asymbatos Foreman, 1968

?Stichomitra campi (Campbell and Clark, 1944a) emend, Foreman, 1968

Stichomitra cathara Foreman, 1968

Stichopilium bicorne Haeckel, 1887

Theocalyptra sp.

?Theocapsa elata Tan Sin Hok, 1927

Theocorys veneris Haeckel, 1887

Theocorys sp. aff. T. antiqua Squinabol, 1903

Theopilium pyramidale Popofsky, 1913 
?Tricolocapsa parvipora Tan Sin Hok, 1927

?Tripocalpis ellyae Tan Sin Hok, 1927

Udan undulata Renz, in press

Subfamily Artostrobiinae Riedel, 1967b

Artostrobium auritum group (Ehrenberg, 1844)

Artostrobium miralestense (Campbell and Clark, 1944a)

Carpocanarium papillosum (Ehrenberg, 1872a)

Lithomitra lineata group (Ehrenberg, 1838)

Siphocampe corbula (Harting, 1863)

Spirocyrtis scalaris Haeckel, 1887

Spirocyrtis sp. aff. S. scalaris Haeckel, 1887

Subfamily Plectopyramidinae Haeckel, 1908, emend. Petrushevskaya, 1971a

Bathropyramis ramosa Haeckel, 1887

Bathropyramis timorensis $\mathrm{n}$. sp.

Bathropyramis sp.

Cinclopyramis infundibulum Haeckel, 1887

?Cornutella californica var. A Campbell and Clark, 1944b, emend. Foreman, 1968

?Cormutella californica var. B Campbell and Clark, 1944b, emend. Foreman, 1968

Cornutella profunda Ehrenberg 1854

Cornutella sp. aff. C. longisetta Ehrenberg, 1872a

?Cyrtocalpis operosa Tan Sin Hok, 1927

Litharachnium tentorium Haeckel, 1862

Subfamily Pterocorydinae Haeckel, 1881, emend. Riedel, 1967a

Anthocyrtidium ophirense (Ehrenberg, 1872a)

Anthocyrtidium zanguebaricum (Ehrenberg, 1872a)

Lamprocyclas maritalis Haeckel, 1887

Lamprocyrtis haysi Kling, 1973

Lamprocyrtis heteroporus (Hays, 1965)

Tetracorethra tetracorethra (Haeckel, 1887) emend. Petrushevskaya, 1971b

Theoconus hertwigii (Haeckel, 1887)

Theoconus junonis Haeckel, 1887

Theocorythium trachelium (Ehrenberg, 1872a)

Subfamily Amphipyndacinae Riedel, 1967b, emend. Petrushevskaya, 1971a

Amphipyndax (?) epiplatys $\mathrm{n} . \mathrm{sp}$.

Amphipyndax mediocris (Tan Sin Hok, 1927)

Amphipyndax (?) pyrgodes $\mathrm{n} . \mathrm{sp}$.

Family Williriedellidae Dumitrica, 1970

?Hemicryptocapsa pseudopilula Tan Sin Hok, 1927

Family Carpocaniidae Haeckel, 1881, emend. Riedel, 1967a

Carpocanistrum spp.

Superfamily Cannobotryoidea Haeckel, 1881, emend. Riedel, 1967a

Family Cannobotryidae Haeckel, 1881, emend. Riedel, $1967 \mathrm{a}$

Acrobotrys sp.

Botryocyrtis quinaria Ehrenberg, 1872a

Botryocyrtis spp.

Botryopyle dictyocephalus Haeckel, 1887

Centrobotrys thermophila Petrushevskaya, 1965 shevskaya, 1971a

Superfamily Plagiacanthoidea Hertwig, 1879, emend. Petru-

shevskaya, 1971 .

Sethophormis pentalactis Haeckel, 1887

Sethophormis rotula Haeckel, 1887

Sethophormis sp. aff. S. pentalactis Haeckel, 1887

Sethophormis sp.

Family Plagiacanthidae Hertwig, 1879, emend. Petrushevskaya, 1971a

Subfamily Lophophaeninae Haeckel, 1881, emend. Petrushevskaya, 1971a

Amphiplecta acrostoma Haeckel, 1887

Dictyophimus tetracanthus Popofsky, 1913

Lamprotripus butschlii (Haeckel, 1887)

Lithomelissa monoceras Popofsky, 1913

Lophophaena cylindrica (Cleve, 1900)

Lophophaena sp.

Lophophaeniid sp. A

Lophophaeniid sp. B

Lophophaeniid sp. C

Lophophaenoma sp. aff. L. witjazii Petrushevskaya, 1971b
Peromelissa phalacra (Haeckel, 1887) emend. Petrushevskaya, 1971b

Pseudodictyophimus gracilipes (Bailey 1856) tetracanthus (Popofsky, 1913)

Psilomelissa calvata Haeckel, 1887

Verticillata hexacantha Popofsky, 1913

Subfamily Sethoperinae Haeckel, 1881, emend. Petrushevskaya, 1971a

Callimitra spp.

Clathrocanium spp.

Clathrocorys sp.

?Pteropilium stratiotes Haeckel, 1887

Suborder Spyrida Ehrenberg, 1847, emend. Petrushevskaya, 1971 a

Family Triospyrididae Haeckel, 1881, emend. Petrushevskaya, 1971a

Cantharospyris platybursa $\mathrm{Haeckel,} 1887$

Ceratospyris sp. aff. C. sp. A

Ceratospyris spp.

Dendrospyris sp. aff. D. binapertonis Goll, 1968

?Dendrospyris sp. aff. D. stabilis Goll, 1968

Giraffospyris laterispina Goll, 1969

Lamprospyris hookeri Haeckel, 1887

Nephrospyris renilla Haeckel, 1887

Tholospyris scaphipes (Haeckel, 1887)

Tholospyris tripodiscus Haeckel, 1887

Tholospyris sp.

Family Acanthodesmiidae Haeckel, 1862, emend. Riedel, 1971

Giraffospyris angulata (Haeckel, 1887)

Liriospyris costata (Haeckel, 1887)

Liriospyris reticulata (Ehrenberg, 1872a)

Liriospyris sp. 1

Liriospyris sp. 2

Tympanidium foliosum Haeckel, 1887

Zygocircus capulosus Popofsky, 1913

Zygocircus sp. $1 \mathrm{cf}$. Z. capulosus Popofsky, 1913

Zygocircus sp. 2 aff. Z. capulosus Popofsky, 1913

Zygocircus sp. aff. Z. piscicaudatus Popofsky, 1913

\section{b. Taxonomic Listing}

(If a dimension represents the mean

of the measurements taken, it is indicated

by a bar above the number, as 63 .)

Acrobotrys sp.

Riedel and Sanfilippo, 1971, pl. 1J, fig. 17, 18.

This chapter: Plate 18, Figure 9; Table 7.

Quaternary.

Actinomma arcadophorum Haeckel, 1887

Renz, in press.

This chapter: Plate 14, Figure 1-; Tables 4, 6, 7.

Quaternary.

Actinommid 1 gen. and sp. indet.

Dimensions: ( 2 specimens) diameter medullary shell $\overline{63} \mu$; diameter cortical shell $\overline{570} \mu$; external primary spine $\overline{87} \mu$; external secondary spine $30 \mu$.

This chapter: Plate 14, Figure 9; Tables 4, 6.

Quaternary.

This chapter: Plate 13, Figure 20; Table 4.

Quaternary.

Actinommid 2 gen. and sp. indet.

Dimensions: (2 specimens) shell diameter $\overline{69} \mu$, spine length $\overline{140} \mu$;

number of arms 12 .

This chapter: Plate 14, Figure 2; Tables 4, 7.

Quaternary.

Actinommid 3 gen. and sp. indet.

Dimensions: ( 2 specimens) diameter medullary shell $\overline{36} \mu$; diameter

cortical shell $\overline{120} \mu$; large spine $\overline{141} \mu$; other spines $45 \mu$.

This chapter: Plate 14, Figure 9; Tables 4, 6.

Quaternary.

Actinommid 4 gen. and sp. indet.

Description: Spherical shell with internal medullary shell from which primary spines arise: these connect to and extend beyond the cortical shell; their three-bladed nature appears only in the extensions and arises when the spines are formed over the margins of pores on the cortical shell; these pores are very large and irregular. 
Dimensions: (2 specimens) diameter medullary shell $\overline{47} \mu$; diameter cortical shell $144-156 \mu$; extended spine $\overline{45} \mu$.

This chapter: Plate 9, Figure 16; Table 5.

Cretaceous.

Amphibrachium (?) hastatum, n. sp.

Description: Hagiastrid with two arms, broad in the central area and tapering into stout three-bladed spines; four parallel rows of pores run the length of the shell.

Dimensions: (6 specimens) shell length $354-397 \mu$; shell width 120

$126 \mu$; spine $105-216 \mu$.

Remarks: Specimens are more often found broken in half. Although the genus Amphibrachium in recent usage has been included in the Spongodiscidae, the type species $A$. diminutum Rust, 1885 , may have been a Hagiastrid. Pessagno, 1971, revised its familial assignment to the Hagiastridae in which it is the only genus for two-rayed species. If this revision proves correct, the genus should be redefined to include species with tapering tips and terminal spines. The specific name is Latin for spear-bearing.

Type locality: $12^{\circ} 56.83^{\prime} \mathrm{S}, 117^{\circ} 53.56^{\prime} \mathrm{E}$; DSDP Leg 27 , Sample 261 12 , CC.

This chapter: Plate 1, Figures 1-6; Plate 9, Figure 1; Tables 3, 5. Cretaceous.

Amphibrachium sp. cf. A. concentricum Lipman, 1960

Riedel and Sanfilippo, 1970, p. 504, pl. 1, fig. 7.

Dimensions: ( 2 specimens) long axis $\overline{114} \mu$; short axis $\overline{75} \mu$.

This chapter: Plate 9, Figure 11; Tables 3, 5 .

Cretaceous.

Amphiplecta acrostoma Haeckel, 1887

Petrushevskaya, 1971b, p. 103, pl. 54, fig. II-IV.

Note: Petrushevskaya does not describe or discuss the complete thorax with a row of double spines which was characteristic of most specimens examined here.

This chapter: Plate 18, Figure 12; Tables 4,6,7.

Quaternary.

Amphipyndax(?) epiplatys n. sp.

Description: Elevated spherical cephalis enveloped in a short pointed apical horn; variable number of segments (8-12); shell conical; pores rounded, slightly irregular, arranged approximately in a checkerboard pattern with two or three rows per segment; pore wall usually thickened around the first two abdominal segments forming "shoulders."

Dimensions: $(2$ specimens) total height $276 \mu(8$ segments $)->420 \mu$

(12 segments); maximum width $144-180 \mu$; apical spine $6-15 \mu$.

Remarks: Although this species possesses the characteristic knoblike cephalis and neck of Amphipyndax, the ring dividing the cephalis was obscured and is only assumed to be present. Specimens were found in Site 149 material from Rotti, but Tan Sin Hok, 1927, does not describe the species. A. epiplatys $\operatorname{differs}$ from $A$. enesseffi and $A$. mediocris by possessing a stout apical horn which envelopes the cephalis and a pore wall thickening around the first two abdominal segments. The specific name is Greek for "broad-topped."

Type locality: $12^{\circ} 56.83^{\prime} \mathrm{S}, 117^{\circ} 53.56^{\prime} \mathrm{E}$, DSDP Leg 27 , Sample 261 $9-4,110-112 \mathrm{~cm}$

This chapter: Plate 5, Figures 1-3; Plate 12, Figure 2; Tables 3, 5; Sections 2 and 3.

Cretaceous.

Amphipyndax mediocris (Tan Sin Hok, 1927)

1927 Dictyomitra mediocris Tan Sin Hok, p. 55, pl. 10, fig. 82.

1968 Amphipyndax stocki (Campbell and Clark, 1944b) emend.

Foreman, p. 78, pl. 8, fig. 12a-c.

Remarks: Material from Site 149, Rotti, was examined to determine the real similarity between the Tan Sin Hok species and the Foreman, 1968, species. The only difference is that specimens from DSDP Leg 27, Site 261 and Rotti, Site 149, tend to have fewer segments (5-7).

This chapter: Plate 5, Figures 7-9; Plate 12, Figures 3; Tables 3, 5; Section 3.

Cretaceous.

Amphipyndax (?) pyrgodes n. sp.

Description: Elevated spherical cephalis enveloped in a short pointed apical horn; variable number of segments (13-17); shell conical; segments with slight thickenings at the constrictions have a scalloped appearance; pores rounded in two-three rows per segment.

Dimensions: ( 3 specimens) total height $399 \mu$ (13 segments) to $540 \mu$

(17 segments); maximum width 153-219 $\mu$; apical spine $\overline{10} \mu$.

Remarks: As in A. epiplatys, the neck area was obscured so that the typical Amphipyndacid ring was not observed.
This species is similar to A. epiplatys, but differs from it by usually possessing more segments, by displaying a thickening of the pore wal at each constriction, and by lacking the "shoulders" or general thickening of the pore wall in the upper segments. This species was not found in material from Site 149, Rotti. The specific name is Greek for tower-like.

Type locality: $12^{\circ} 56.83^{\prime} \mathrm{S}, 117^{\circ} 53.56^{\prime} \mathrm{E}$, DSDP Leg 27 , Sample 261$12-2$, top.

This chapter: Plate 5, Figures 4-6; Plate 12, Figure 1; Tables 1, 3, 5; Section 2b, c.

Cretaceous.

Amphirhopalum ypsilon Haeckel, 1887

Nigrini, 1967, p. 35, pl. 3, fig. 3a-d.

This chapter: Plate 15, Figure 14; Tables 2, 4, 6, 7, 8 ,

Quaternary.

Anthocyrtidium ophirense (Ehrenberg, 1872a)

Renz, in press.

This chapter: Plate 19, Figure 22; Tables 2, 4, 6, 7, 8 .

Quaternary.

Anthocyrtidium zanguebaricum (Ehrenberg, 1872a)

Renz, in press.

This chapter: Plate 19, Figure 17; Tables 2, 4, 6, 7.

Quaternary.

Artocapsa bicornis Tan Sin Hok, 1927

Tan Sin Hok, 1927, p. 74, pl. 16, fig. 142

?Stichomitra livermorensis (Campbell and Clark, 1944b) in Foreman, 1968, p. 76, pl. 8, fig. 2a only.

Description: Cephalis rounded but irregular with stout apical spine; shell conical with six segments rounded at the bottom with a stout three-bladed terminal spine; pore wall relatively thick with spiny protuberances; pores hexagonally rounded.

Dimensions: ( 2 specimens) total height $\overline{234} \mu$; maximum width $\overline{105} \mu$; terminal spine $48-66 \mu$; apical spine $\overline{18} \mu$; pore wall $14 \mu$.

Remarks: This species was not found at Site 149, Rotti.

This chapter: Plate 6, Figures 21-23; Plate 11, Figures $12 \mathrm{a}$, b;

Tables 3, 5 .

Cretaceous.

Artocapsa ultima Tan Sin Hok, 1927

Tan Sin Hok, 1927 , p. 74 , pl. 16, fig. 143.

?Stichomitra livermorensis (Campbell and Clark, 1944b) in Foreman, 1968, p. 76, pl. 8, fig. 2b only.

Description: Cephalis irregular with stout apical spine and several secondary spines; shell conical with six segments, rounded at the bottom with a stout three-bladed terminal spine; pores large, rounded, arranged in rows in checkerboard fashion.

Dimensions: (2 specimens) total height $\overline{342 \mu}$; maximum width $156 \mu$; pore wall $\overline{18} \mu$; apical spine $\overline{45} \mu$.

Remarks: This species differs from the concept of Tan Sin Hok in the fact that there are six rather than seven segments and the last is the broadest rather than the fifth; the pores are also larger and more regular. This species was not found in the material from Site 149, Rotti.

This chapter: Plate 6, Figure 24; Plate 11, Figure 13; Tables 3, 5. Cretaceous.

Archipilium sp. aff. A. macropus (Haeckel, 1887)

Petrushevskaya and Kozlova, 1972, p. 553, pl. 29, fig. 13, 14

Remarks: Although Petrushevskaya and Kozlova, 1972, found similar specimens in Miocene-Pliocene material, the specimens observed here are believed to be much younger.

This chapter: Plate 17, Figures 11a, b; Tables 4, 6 .

?Quaternary-Pleistocene.

Artopilium sp. aff. A. elegans Haeckel, 1887

Haecker, 1887 , p. 1440, pI. 75, fig. 1.

Remarks: The species has a more spherical cephalis with a single apical horn and feet which turn convexly inward; spines usually occur only on the thoracic portion of the feet.

This chapter: Plate 17, Figure 4; Tables 4, 6, 7.

Quaternary.

Artopilium undulatum Popofsky, 1913

Renz, in press.

Remarks: Most specimens have four-six segments.

This chapter: Plate 16, Figure 13; Tables 4, 6, 7.

Quaternary.

Artostrobium auritum group (Ehrenberg, 1844)

Renz, in press.

This chapter: Plate 17, Figure 13; Tables 2, 4, 6, 7, 8 .

Quaternary. 
Artostrobium miralestense (Campbell and Clark, 1944a).

Renz, in press.

This chapter: Plate 17, Figure 22; Tables 2, 4, 6, 7

Quaternary.

Astrosphaera hexagonalis Haeckel, 1887

Renz, in press.

This chapter: Plate 14, Figure 1; Table 7.

Quaternary.

Bathropyramis ramosa Haeckel, 1887

Haeckel, 1887, p. 1161, pl. 54, fig. 4.

Dimensions: ( 2 specimens) length $480-495 \mu$; width $186-213 \mu$; rib width $9-15 \mu$; cephalis $12-15 \mu$.

This chapter: Plate 17, Figure 27; Tables 4, 7

Quaternary.

Bathropyramis timorensis $\mathbf{n}$. $\mathbf{s p}$.

Description: Plectopyramid with nine very thick, wing-like ribs which in cross-section appear as points on a star; these flare in the upper portion as "shoulders"; pores between ribs are single and elliptical in the upper portion becoming rounded when double or triple; cephalis simple, difficult to see, encased in stout apical spine.

Dimensions: (6 specimens) total length $279-345 \mu$; maximum width

117-231 $\mu$; maximum width between ribs $45-75 \mu$; rib thickness $\overline{32} \mu$; apical spine $\overline{66} \mu$; rib thickness at "shoulder" $\overline{27} \mu$.

Remarks: This species differs from other species of Bathropyramis in its very characteristic wing-like ribs which possess an upper thoracic thickening or shoulders. This species is named for the Island of Timor near which it was discovered.

Type locality: $12^{\circ} 56.83^{\prime} \mathrm{S}, 117^{\circ} 53.56^{\prime} \mathrm{E}$, DSDP Leg 27, Sample 261-

9-4, $110-112 \mathrm{~cm}$.

This chapter: Plate 4, Figures 8, 9; Plate 12, Figure 4 a-c; Tables 3,

5; Sections 2c, 3, 4

Cretaceous.

Bathropyramis sp.

Similar to Renz, in press.

Remarks: apical horns never observed unbroken.

This chapter: Plate 17, Figure 26; Tables 4, 6 .

Quaternary.

Botryocyrtis quinaria Ehrenberg, 1872a

Petrushevskaya and Kozlova, 1972, p. 554, pl. 39, fig. 7; Section 2b.

This chapter: Plate 18, Figure 19; Tables 2, 4, 7.

Quaternary.

Botryocyrtis spp.

Renz, in press.

This chapter: Plate 18, Figure 15; Tables 2, 4, 6, 7.

Quaternary.

Botryopyle dictyocephalus Haeckel, 1887

Petrushevskaya, 1971b, p. 161, pl. 83, fig. I-V.

This chapter: Plate 18, Figures 10, 20; Tables 2, 4, 6, 7.

Quaternary.

Callimitra spp.

Renz, in press.

This chapter: Plate 18, Figure 5; Tables 4, 7.

Quaternary.

Calocyclas monumentum Haeckel, 1887

Renz, in press.

This chapter: Plate 16, Figure 25; Tables 4, 6, 7 .

Quaternary.

Cantharospyris platybursa Haeckel, 1887

Renz, in press.

This chapter: Plate 19, Figures 19a, b; Tables 4, 7.

Quaternary.

Carpocanarium papillosum (Ehrenberg, 1872a)

Riedel and Sanfilippo, 1971, p. 132, pl. 11, fig. 24.

This chapter: Plate 17, Figure 21; Tables 4, 6, 7; Sections 2b, c. Quaternary.

Carpocanistrum spp.

Renz, in press.

This chapter: Plate 17, Figure 17; Tables 2, 4, 6, 7.

Quaternary.

Centrobotrys thermophila Petrushevskaya, 1965

Nigrini, 1967, p. 49, pl. 5, fig. 7.

This chapter: Plate 18, Figure 16; Tables 2, 4, 6, 7; Section 2c.

Quaternary.

Centrocubus octostylus Haeckel, 1887

Haeckel, 1887, p. 278 , pl. 18, fig. 1.

This chapter: Plate 14, Figure 14; Tables 4, 6, 7.

Quaternary.
Ceratospyris sp. aff. $\underline{\text { C. }}$ sp. A

Renz, in press.

Remarks: The polygonal pores with meshwork at the lateral extremes of the bilocular cephalis are not as bulbous in these specimens.

This chapter: Plate 19, Figure 8; Tables 4, 6, 7.

Quaternary.

Ceratospyris spp.

Renz, in press.

This chapter: Plate 19, Figure 9; Tables 4, 6, 7.

Quaternary.

Cinclopyramis infundibulum Haeckel, 1887

Renz, in press.

This chapter: Plate 17, Figure 23; Tables 2, 4, 6, 7, 8.

Quaternary.

?Cladococcus abietinus Haeckel, 1887

Haeckel, 1887, p. 226 , pl. 27, fig. 3.

Remarks: This species differs from Haeckel's description in

possessing spines dentated only toward the tips.

This chapter: Plate 13, Figure 18; Tables 4, 6, 7.

Quaternary.

?Cladococcus pinetum Haeckel, 1887

Haeckel, 1887, p. 26 , pl. 27, fig. 1.

Dimensions: (2 specimens) diameter of sphere $\overline{99} \mu$; spine $\overline{240 \mu}$.

Remarks: The assignment of this species is tentative because the

branching of the spines is less dramatic than described by Haeckel.

This chapter: Plate 13, Figure 19; Table 7.

Quaternary.

Cladococcus scoparius Haeckel, 1887

Haeckel, 1887 , p. 225 , pl. 27, fig. 2.

Dimensions: (2 specimens) diameter medullary shell $60 \mu$; spine

$120 \mu$; number of spines $>22$.

This chapter: Plate 13, Figure 17; Tables 4, 7.

Quaternary.

Cladococcus sp.

Dimensions: ( 3 specimens) shell diameter $\overline{75} \mu$; spine length $5 T \mu$;

number of arms $>18$.

This chapter: Plate 14, Figure 4; Table 7.

Quaternary.

Clathrocanium spp.

Renz, in press.

This chapter: Plate 18, Figure 3; Tables 2, 4, 6, 7.

Quaternary.

Clathrocyclas semeles Haeckel, 1887

Haeckel, 1887, p. 1388, pl. 58, fig. 5 .

This chapter: Plate 16, Figure 16; Tables 4, 6, 7.

Quaternary.

Clathrocorys sp.

Renz, in press.

This chapter: Plate 18, Figure 4; Tables 4, 6, 7.

Quaternary.

Collosphaera polygona Haeckel, 1887

Renz, in press.

This chapter: Plate 13, Figure 14; Tables 2, 4, 6, 7.

Quaternary.

Collosphaera tuberosa Haeckel, 1887

Nigrini, 1971, p. 445, pl. 34.1, fig. 1.

This chapter: Plate 13, Figure 6; Tables 6, 7.

Quaternary,

?Conosphaera tuberosa Tan Sin Hok, 1927

Tan Sin Hok, 1927 , p. 36 , pl. 6, fig. 10.

Dimensions: ( 2 specimens) radius $\overline{90} \mu$; radius of medullary shell $24 \mu$.

Remarks: This species is similar to that described by Tan Sin Hok but smaller. It was found at Site 149, Rotti.

This chapter: Plate 2, Figures 6-8; Plate 9, Figure 17; Tables 3, 5. Cretaceous.

?Cornutella californica var. A Campbell and Clark, 1944b, emend. Foreman, 1968

Foreman, 1968, p. 21, pl. 3, fig. Ic.

Dimensions: ( 2 specimens) length without horn $108 \mu$; maximum width $90 \mu$; apical horn $153 \mu$.

Remarks: This species is similar to that figured by Foreman but tends to have a shorter broader thorax. No similar species was described by Tan Sin Hok, 1927.

This chapter: Plate 4, Figure 10; Plate 12, Figure 6; Table 5.

Cretaceous. 
?Cornutella californica var. B Campbell and Clark, 1944a, emend. Foreman, 1968

Foreman, 1968, p. 21, pl. 3, fig. 1b.

Dimensions: (2 specimens) total length $\overline{240} \mu$; maximum width $\overline{45} \mu$; apical horn $84 \mu$.

Remarks: Two similar Cornutella species occur at Site 149, Rotti and were described by Tan Sin Hok, 1927, as C. apicata and C. facilis. In both, the characteristics of the pores and the pore wall differ sufficiently from this species to justify another name. Although $C$. californica has been described as Maestrichtian from the eastern Pacific, it is at least more superficially similar to this species than the others.

This chapter: Plate 4, Figure 11; Plate 12, Figure 7; Table 5.

Cretaceous.

Cornutella profunda Ehrenberg 1854a

Nigrini, 1967, p. 60 , pl. 6 , fig. 5a-c, fig. $5 \mathrm{a}=$ var. B, $5 \mathrm{c}=$ var. $\mathrm{A}$.

Remarks: Nigrini regarded both thin- and thick-walled forms as conspecific. These have been separated for abundance estimates into two varieties, due to the fact that no transitional forms were observed.

This chapter: Plate 17, Figures 24, 25; Tables 2, 4, 6, 7 (var A);

Tables 4, 6, 7 (var B).

Quaternary.

Cornutella sp. aff. C. Iongisetta Ehrenberg, 1872a

Petrushevskaya and Kozlova, 1972, p. 551, pl. 30, fig. 8.

Remarks: Specimens in Leg 27 material have a thicker shell wall and hexagonal pores.

This chapter: Plate 17, Figure 20; Table 6.

Quaternary.

Corocalyptra cervus (Ehrenberg, 1872b)

Renz, in press.

This chapter: Plate 16, Figure 22; Tables 4, 6, 7.

Quaternary.

Corocalyptra killmari Renz, in press

Renz, in press.

This chapter: Plate 17, Figure 10; Tables 4, 7.

Quaternary.

Corocalyptra kruegeri Popofsky, 1908

Renz, in press.

This chapter: Plate 16, Figure 10; Table 7.

Quaternary.

Corocalyptra sp. aff. C. kruegeri Popofsky, 1908

Renz, in press.

Remarks: Specimens of this type differ from the original species

description by forming postabdominal segments of an undulating

form rather than by true constrictions.

This chapter: Plate 16, Figure 11; Table 7.

Quaternary.

Crucella espartoensis Pessagno, 1971

Pessagno, 1971, p. 54, pl. 18, fig. 1-4.

Remarks: Usually without a patagium.

This chapter: Plate 1, Figures 11, 12; Plate 9, Figure 4; Tables 3, 5;

Section 3

?Cyclampterium neatum Sanfilippo and Riedel, 1970

Riedel and Sanfilippo, 1971, p. 106, pl. 1E, fig. 4-6.

This chapter: Plate 16, Figure 29; Table 4.

Quaternary.

?Cyrtocapsa grutterinki var. $\alpha$ Tan Sin Hok, 1927

Tan Sin Hok, 1927 , p. 65, pl. 13, fig. 111.

Dimensions: ( 2 specimens) total height without apical or terminal spines $193-231 \mu$; thorax length $\overline{27} \mu$; abdomen length $\overline{142} \mu$; maximum width $179 \mu$; terminal spine $36-75 \mu$.

Remarks: Cephalis may have three or more short apical spines or one stout three-bladed spine. This species differs from that of Tan Sin Hok by possessing only one abdominal segment.

This chapter: Plate 6, Figures 1-3; Plate 11, Figure 7; Tables 1, 3, 5; Section 3.

?Cyrtocalpis operosa Tan Sin Hok, 1927

Tan Sin Hok, 1927 , p. 40 , pl. 7, fig. 27.

Description: Appearing to be a "pseudo cornutella" with a tapering thorax, spherical cephalis with small apical horn; pores rounded in alternating vertical rows and moderately close set.

Dimensions: ( 2 specimens) total height $123-210 \mu$; maximum width $66 \mu$; apical spine $6 \mu$; pore wall $9-12 \mu$.

Remarks: This species differs from the concept of Tan Sin Hok in being taller and having a thinner pore wall.
This chapter: Plate 4, Figures 15, 16; Plate 12, Figure 8; Tables 1, 3,

5; Section 3.

Cretaceous.

Cyrtopera sp. aff. C. languncula Haeckel, 1887

Renz, in press.

Remarks: All specimens are broken.

This chapter: Plate 16, Figure 2; Tables 4, 6, 7.

Quaternary.

Dendrospyris sp. aff. D. binapertonis Goll, 1968

Goll, 1972 , p. 963 , pl. 49 , fig. 1,2 .

Dimensions: ( 4 specimens) height $\overline{60} \mu$; width $\overline{87} \mu$; "feet" $\overline{36} \mu$.

This chapter: Plate 19, Figure 11; Tables 4, 6, 7.

Quaternary.

?Dendrospyris sp. aff. D. stabilis Goll, 1968

Renz, in press.

This chapter: Plate 19, Figure 14; Tables 2, 4, 6, 7.

Quaternary.

Diacanthocapsa sp. B

Petrushevskaya and Kozlova, 1972, p. 536, pl. 7, fig. 4, 5.

Dimensions: ( 2 specimens) height $141 \mu$; maximum width $96 \mu$; abdominal wall thickness $18 \mu$.

This chapter: Plate 11, Figure 18; Table 5.

Cretaceous.

Dicolocapsa verbeeki Tan Sin Hok, 1927

Tan Sin Hok, 1927, p. 44, pl. 8, fig. 40 .

1968 ? Solenotryma sp. Foreman, p. 33, pl. 4, fig. 6.

Dimensions: (4 specimens) height $\overline{104} \mu$; maximum width $\overline{93} \mu$; pore wall $9 \mu$.

Remarks: It appears that species in the Tricolocapsa parvipora group are related to $D$. verbeeki since both show a tendency to grow septa (internal constrictions) indiscriminately in the thorax. A terminal tube as described by Foreman was never observed.

This chapter: Plate 6, Figures 13-18; Plate 11, Figure 9; Tables 1, 3, 5.

Cretaceous.

Dicolocapsa sp.

Description: Cephalis subspherical without apical spine, thorax subspherical, third segment (?) or "skirt" only rudimentary yet always present; pores very small, regular and wide-set.

Dimensions: (3 specimens) height $\overline{162} \mu$; width $\overline{108} \mu$; "skirt" length $\overline{54} \mu$; pore wall $\overline{12} \mu$.

This chapter: Plate 6, Figure 19; Plate 11, Figure 2; Table 5.

Cretaceous.

Dictyoceras virchowii Haeckel, 1862

Haeckel, 1862 , pl. 8, figs. 6-8.

This chapter: Plate 17, Figure 5; Tables 4, 6, 7.

Quaternary.

Dictyocodon palladius Haeckel, 1887

Haeckel, 1887, p. 1335, pl. 71, fig. 12, 13.

This chapter: Plate 16, Figure 1; Table 7.

Quaternary.

Dictyocoryne profunda Ehrenberg, 1872b

Renz, in press.

This chapter: Plate 15, Figure 7; Tables 2, 4, 6, 7, 8; Sections 2b-d. Quaternary.

Dictyomitra brouweri var. $\alpha$ (Tan Sin Hok, 1927)

1927 Eucyrtidium brouweri var. $\alpha$ Tan Sin Hok, p. 58, pl. 11, fig. 93. Moore, 1973, pl. 14, fig. 7-9.

Remarks: Genus revised. See D. brouweri var. $\gamma$. Specimens found at Site 149, Rotti.

This chapter: Plate 8, Figures 14-16; Plate 11, Figure 26; Tables 1, 3, 5; Sections 2b-d, 3.

Cretaceous.

Dictyomitra brouweri var. $\gamma$ (Tan Sin Hok, 1927)

1927 Eucyrtidium brouweri var. $\gamma$ Tan Sin Hok, p. 58, pl. 11, fig. 91.

Remarks: Specimens do not have the thickened pore walls as illustrated by Tan Sin Hok. The generic assignment has been revised because this species appears to be more closely related to the type species of Dictyomitra, i.e., D. multicostata Zittel, 1876, than to the type species of Eucyrtidium, i.e., E. acuminatum Ehrenberg, 1847; specimens found at Site 149, Rotti.

This chapter: Plate 8, Figures 9-13; Plate 11, Figure 27; Tables 1, 3, 5; Sections 2b-d, 3.

Cretaceous. 
Dictyomitra excellens (Tan Sin Hok, 1927)

1927 Lithomitra excellens Tan Sin Hok, p. 56, pl. 11, fig. 85. Dimensions: ( 3 specimens) total length $225-375 \mu$; maximum width $102 \mu$.

Remarks: Number of segments varies from 10 to 13 . The generic assignment of this species has been reevaluated because it appears to be more closely related to the type species Dictyomitra, i.e., D. multicostata Zittel, 1876, than to the type species of Lithomitra, i.e., $L$. pachyderma Ehrenberg, 1873, probably an artostrobiid.

This chapter: Plate 8, Figures 7, 8; Plate 11, Figure 35; Tables 1, 3,

Cretaceous

Dictyomitra lilyae Tan Sin Hok, 1927

Tan Sin Hok, 1927, p. 56, pl. 10, fig. 83 .

Dimensions: ( 3 specimens) total length $\overline{207} \mu$; maximum width

$\overline{106} \mu$; length largest segment $30 \mu$.

Remarks: Several specimens of D. lilyae were found in the material from Sites 149 and 384, Rotti. This species bears a strong resemblance to $D$. formosa Squinabol, 1904, as presented in Moore, 1973, and to $D$. haeckelii (Pantanelli, 1880) as presented in Hinde, 1900.

This chapter: Plate 8, Figures 1-4; Plate 11, Figure 33; Tables 3, 5.

Sections $2 \mathrm{~b}$, c.

Cretaceous.

?Dictyomitra malleola Aliev, 1961

Aliev, 1965 , p. 48 , pl. 8 , fig. 6

Dimensions: ( 3 specimens), height $\overline{357} \mu$, width $\overline{141} \mu$; number of segments 14-15.

Remarks: The swelling of the second abdominal segment is not as great as pictured by Aliev.

This chapter: Plate 8, Figure 20; Plate 11, Figure 28; Table 5. Cretaceous.

Dictyomitra pseudoscalaris (Tan Sin Hok, 1927)

1927 Stichomitra pseudoscalaris Tan Sin Hok, p. 56, pl. 11, fig. 84.

Dimensions: ( 3 specimens), total length $\overline{251} \mu$; maximum width

$110 \mu$; length largest segment $\overline{45} \mu$.

Remarks: Number of segments varies from 6 to 10; a small apical horn may or may not be present. The generic assignment of this species has been reevaluated because it appears from the arrangement of the pores and costae to be more closely related to the type species of Dictyomitra, i.e., D. multicostata Zittel, 1876, than to the type species of Stichomitra, S. costata Cayeux, 1897.

This chapter: Plate 8, Figures 5, 6; Plate 11, Figure 34; Tables 1, 3, 5; Sections 2b, c.

Cretaceous.

Dictyophimus crisiae Ehrenberg, 1854

Nigrini, 1967, p. 66 , pl. 6 , fig. $7 \mathrm{a}, \mathrm{b}$.

This chapter: Plate 17, Figure 2; Tables 4, 6, 7 .

Quaternary.

Dictyophimus gracilis Tan Sin Hok, 1927

Tan Sin Hok, 1927, p. 42, pl. 7, fig. 33 .

Description: Cephalis hemispherical with stout, flared, threepronged apical spine; thorax hemispherical with three stout, threebladed legs extending from its edge and curving inward; pores moderately large and irregularly hexagonal.

Dimensions: ( 3 specimens) length (cephalis and thorax) 63-81 $\mu$; apical spine $35-42 \mu$; leg $49-60 \mu$; maximum thorax width $75-78 \mu$.

Remarks: Material from Site 149, Rotti, was examined to insure the similarity between the species described by Tan Sin Hok and the species in DSDP Site 261 material.

This chapter: Plate 5, Figures 14-16; Plate 11, Figure 11; Table 5; Sections 2c, 3 .

Cretaceous.

Dictyophimus obliquum (Hinde, 1900)

(1900 Tripilidium obliquum Hinde, p. 26, pl. 2, fig. 9.

Description: Eucyrtid with subspherical cephalis and tall, stout, three-bladed apical horn; thorax pear-shaped; from its edge extend three long convex feet with short spines or teeth along the upper inner side.

Dimensions: ( 2 specimens) apical spine $\overline{66} \mu$; length cephalis + thorax $\overline{84} \mu$; maximum width $84 \mu$; length feet $\overline{135} \mu$.

Remarks: The generic assignment of this species has been shifted because this author feels that specimens are more closely related to the type species of Dictyophimus, i.e., D. crisiae Ehrenberg, 1854, established in the argument presented by Nigrini, 1968, than to the type species of Tripilidium, i.e., $T$. nanum Rust, 1885. D. obliquum is similar to $D$. gracilis, but differs from it by the shape of the thorax and the length of the apical horn and feet.

This chapter: Plate 5, Figure 17; Plate 11, Figure 1; Table 5.

Cretaceous.

Dictyophimus tetracanthus Popofsky, 1913

Renz, in press.

This chapter: Plate 18, Figure 11; Tables 4, 7.

Quaternary.

Dictyophimus? sp. indet.

cf. Pseudodictyophimus? sp, indet.

Petrushevskaya and Kozlova, 1972, pl. 37, fig. 22.

This chapter: Plate 17, Figure 18; Tables 4, 7.

Quaternary.

Disolenia quadrata (Ehrenberg, 1872a)

Renz, in press.

This chapter: Plate 13, Figure 8; Tables 2, 4, 6, 7.

Quaternary.

Disolenia zanguebarica (Ehrenberg, 1872a)

Rena, in press.

This chapter: Plate 13, Figure 5; Tables 4, 6, 7, 8 .

Quaternary.

Elatomma sp.

Dimensions: ( 2 specimens) diameter cortical shell $\overline{252} \mu$; diameter medullary shell $\overline{66} \mu$; primary spine length external $\overline{33} \mu$; secondary spine length external $\overline{9}_{\mu}$.

This chapter: Plate 13, Figure 16; Tables 4, 7.

Quaternary.

Euchitonia mulleri Haeckel, 1862

Nigrini, 1967, p. 37, pl. 4, fig. 1a, b.

This chapter: Plate 15, Figure 1; Tables 2, 4, 6, 7, 8; Sections 2b-d. Quaternary.

Eucyrtid 1 gen. and sp. indet.

Dimensions: (1 specimen) cephalis $\overline{18} \mu$; apical spine $\overline{51} \mu$; thorax

length $\overline{105} \mu$; thorax width $\overline{165} \mu$; feet $\overline{30} \mu$ (broken).

This chapter: Plate 16, Figure 15; Tables 4, 6.

Quaternary.

Eucyrtid 2 gen. and sp. indet.

Dimensions: ( 2 specimens) cephalis $\overline{18} \mu$; apical spines $\overline{45-54} \mu$;

thorax length $\overline{126} \mu$; thorax width $\overline{180} \mu$; foot $\overline{186} \mu$.

This chapter: Plate 17, Figure 1; Table 7.

Quaternary.

Eucyrtid 3 gen. and sp. indet.

This chapter: Plate 16, Figure 23; Table 7.

Quaternary.

Eucyrtidium acuminatum (Ehrenberg, 1844)

Renz, in press.

This chapter: Plate 16, Figure 8; Tables 4, 6, 7.

Quaternary.

Eucyrtidium anomalum Haeckel, 1862

Renz, in press.

This chapter: Plate 16, Figure 20; Tables 2, 4, 6, 7.

Quaternary.

Eucyrtidium (?) boodes n. sp.

Description: Multisegmented Eucyrtid with a large subspherical cephalis enveloped in a stout apical horn; shell concical; pore wall thickened around the upper segments forming "shoulders" pores rounded, regular, moderately large.

Dimensions: (2 specimens) cephalis $\overline{21} \mu$; apical horn $\overline{48} \mu$; total length ( 8 segments) $345 \mu$; maximum width $\sqrt{26} \mu$; "shoulder" width $105 \mu$; pore wall at "shoulders" $\overline{30} \mu$.

Remarks: This species is only tentatively assigned to the genus Eucyrtidium since all specimens are broken. It differs from other species in this genus by its characteristic large cephalis and horn and the thickened pore wall around the upper segments. The species name is Greek for ox-like.

Type locality: $12^{\circ} 56.83^{\prime} \mathrm{S}, 117^{\circ} 53.56^{\prime} \mathrm{E}$, DSDP Leg 27 , Sample 261$9-4,110-112 \mathrm{~cm}$

This chapter: Plate 8, Figures 21, 22; Plate 11, Figures 15 a, b; Table

5: Sections 2c, 3.

Cretaceous.

Eucyrtidium hexagonatum Haeckel, 1887

Renz, in press.

This chapter: Plate 16, Figure 3; Tables 2, 4, 6, 7 .

Quaternary. 
Eucyrtidium hexastichum (Haeckel, 1887)

Renz, in press.

This chapter: Plate 16, Figure 6; Tables 2, 4, 6, 7, 8 .

Quaternary.

Eucyrtidium vermiculatum n. sp.

Description: Cephalis small, subspherical with small apical spine; shell slightly conical becoming cylindrical distally. Strong internal septa; pores very irregular with irregular raised ridges running over each segment such that each segment in profile appears to have a bulge or node at its center.

Dimensions: ( 3 specimens) height $153-219 \mu$; width $78-90 \mu$; number of segments six to nine.

Remarks: This species differs from other Cretaceous species of Eucyrtidium by possessing raised irregular ridges which cause intra segmental bulges.

The species name is Latin for worm-like.

Type locality: $12^{\circ} 56.83^{\prime} \mathrm{S}, 117^{\circ} 53.56^{\prime} \mathrm{E}$. DSDP Leg 27 , Sample 261 12-2, top.

This chapter: Plate 8, Figures 17-19; Plate 11, Figure 22; Table 5; Section 3.

Cretaceous.

Eucyrtidium sp. cf. E. thiensis Tan Sin Hok, 1927

Only as figured by Moore, 1973, pl. 7, fig. 6 not Tan Sin Hok, 1927.

Description: Eucyrtid with six segments; small, spherical cephalis without apical horn; shell conical with bulbous end segment; pores small widely spaced in columns.

Dimensions: (2 specimens) total length $\overline{195} \mu$; maximum width $\Pi_{11} \mu$

Remarks: This species has not been found in the material from Site 149, Rotti; after studying the material, it appears that Tan Sin Hok was describing a different species, namely $E$. thiensis discussed in this chapter and not the species designated by Moore.

This chapter: Plate 11, Figure 23; Tables 3, 5; Section 3.

Cretaceous.

\section{Eucyrtis bulbosus n. sp.}

Description: Cephalis subspherical with short apical spine; thorax and abdomen conical with a bulbous spiny lower segment and a pseudosegmented tapering tail; number of segments vary from six to nine; pores hexagonal, closely spaced in alternating rows.

Dimensions: ( 3 specimens) total height $192-309 \mu$; maximum width 96-105 $\mu$; length of largest segment 78-96 $\mu$; length of tail $111 \mu$.

Remarks: This species is quite similar to $E$. hanni, but differs from it by possessing a large bulbous abdominal segment. Many times the tail is broken. The specific name is Latin for bulbous.

Type locality: $12^{\circ} 56.83^{\prime} \mathrm{S}, 117^{\circ} 53.56^{\prime} \mathrm{E}$, DSDP Leg 27 , Sample 261 $12-2,11-12 \mathrm{~cm}$

This chapter: Plate 7, Figures 26-29; Plate 12, Figure 15 a, b; Tables 3, 5; Sections 2b, c, 3 .

Cretaceous.

\section{Eucyrtis columbarius n. sp.}

Description: Cephalis subspherical, sometimes enveloped in a stout apical horn usually offset, at other times simple with no horn; thorax somewhat rounded and hemispherical, abdominal segments, cylindrical or slightly conical with strictures appearing as internal rings; two or more slight lateral, internal ribs on abdomen occur randomly and sometimes protrude at the mouth as a tube; rarely the termina segment appears bulbous and closed; often the lower segments curve out as if the axis is bent; pores round, regular, close set in rows or columns along the internal ribs; those not located on ribs tend to occur in checkerboard fashion.

Dimensions: (6 specimens) height without apical horn $165-237 \mu$; maximum width $72-105 \mu$; number of segments $6-10$.

Remarks: This species is probably related to Eucyrtidium thiensis Tan Sin Hok, 1927 (not Moore, 1973), a description based on one specimen. After studying topotypic material from Site 149, Rotti, two specimens plus fragments were found and considered to be $E$. thiensis. These differ from this species in being more conical in shape and in lacking a bent axis. There is also a similarity based on the character of the pores and internal septa with Cyrtocapsa ovalis Rüst, 1885. Whether this is conspecific with either of the other species can be decided only when topotypic material is available. The specific name is Latin for dove-like.

Type locality: $12^{\circ} 56.83^{\prime} \mathrm{S}, 117^{\circ} 53.56^{\prime} \mathrm{E}$, DSDP Leg 27 , Sample 261 $12-2,11-12 \mathrm{~cm}$
This chapter: Plate 7, Figures 14-20; Plate 12, Figures 13 a-c; Tables 1, 3, 5; Sections 2b, c, 3, 4 . Cretaceous.

Eucyrtis hanni (Tan Sin Hok, 1927)

1927 Lithocampe hanni Tan Sin Hok, p. 64, pl. 13, fig. 109.

Dimensions: ( 4 specimens) total height $189-309 \mu$; length terminal segment $54-87 \mu$; maximum width $66-84 \mu$; apical spine $18-27 \mu$; number of segments 7-11.

Remarks: This species may be conspecific with $E$. zhamoidai Foreman, 1973, and with Stichocapsa bicacuminata Rust, 1885, but until topotypic material can be examined, this cannot be determined. $E$. hanni is present at Site 149 , Rotti. The generic assignment has been revised since this species appears to be more closely related to Eucyrtis conoidea Rust, 1885 (type specimen designated by Foreman, 1973) than to Lithocampe radicula Ehrenberg, 1838.

This chapter: Plate 7, Figures 21-25; Plate 12, Figures 16a, b; Tables 3, 5; Sections 2b, c, 3 .

Cretaceous.

Eucyrtis kruizingai (Tan Sin Hok, 1927)

Tan Sin Hok, 1927, p. 61, pl. 11, fig. 97.

Dimensions: (2 specimens) total height $117-219 \mu$; maximum width $72-99 \mu$; number of segments 4-6.

Remarks: The generic assignment of this species has been revised since it appears to be more closely related to the type species of Eucyrtis, i.e., E. conoidea Rüst, 1885 (designated by Foreman, 1973) than to the type species of Eusyringium, i.e., E. conosiphon Haeckel, 1887.

This chapter: Plate 11, Figure 24; Table 5; Section 3.

Cretaceous.

Eucyrtis molengraaffi (Tan Sin Hok, 1927)

1927 Syringium molengraaffi Tan Sin Hok, p. 63, pl. 13, fig. 105; pl 11, fig. 32 .

Dimensions: ( 3 specimens) total height $123-219 \mu$; maximum width $69-96 \mu$; number of segments

Remarks: This species differs from the concept of Tan Sin Hok in the following ways: (1) number of segments varies from five to eight; (2) segments generally have three rows of pores rather than two. Since this species has little in common with Syringium vinassai Principi, 1909, from the middle Miocene of Tripoli, the type species of this genus, its generic assignment has been revised to Eucyrtis, type species $E$. conoidea Rüst, 1885, designated by Foreman, 1973.

This chapter: Plate 7, Figures 1-4; Plate 11, Figure 32; Tables 1, 3,

Cretaceous.

?Eusyringium siphonostoma Haeckel, 1887

Haeckel, 1887 , p. 1499, pl. 80, fig. 14

Description: Cephalis hemispherical with small apical horn; collar constriction slight; first abdominal segment elongated; pores small, rounded, irregular, and close-set.

Dimensions: (2 specimens) height ( 5 segments) $\overline{162} \mu$; width $\overline{66 \mu}$; thorax $\overline{2 T} \mu$; first abdominal segment $\overline{54} \mu$; second abdominal segment $39 \mu$.

Remarks: Assignment of this species is tentative because all specimens are incomplete; a terminal tube was never observed.

This chapter: Plate 16, Figure 7; Tables 4, 7

Quaternary.

Giraffospyris angulata (Haeckel, 1887)

Renz, in press.

This chapter: Plate 19, Figure 10; Tables 2, 4, 6, 7, 8 .

Quaternary.

Giraffospyris laterispina Goll, 1969

Goll, 1972, p. 965 , pl. 66, fig. 1-4

Dimensions: ( 2 specimens) height $\overline{75} \mu$; width $\overline{96} \mu$; feet $\overline{12} \mu$.

This chapter: Plate 19, Figure 7; Tables 2, 4, 6, 7, 8.

Quaternary.

Hagiastrid 1 gen. and sp. indet.

Dimensions: ( 3 specimens) maximum radius $102-174 \mu$.

Remarks: A similar specimen is pictured in Riedel and Sanfilippo, 1970, pl. 2, fig. 4

This chapter: Plate 1, Figures 7, 8; Plate 9, Figure 3; Tables 3, 5; Section 3.

Cretaceous.

Hagiastrid 2 gen. and sp. indet.

Dimensions: ( 2 specimens) arm $\overline{47} \mu$; central disc $\overline{60} \mu$, 
This chapter: Plate 1, Figures 9, 10; Plate 9, Figure 6; Tables 3, 5. Cretaceous.

?Halesium quadratum Pessagno 1971

Pessagno, 1971, p. 23, pl. 3, fig. 1-6; pl. 4, fig. 1, 2.

Remarks: Specimens are smaller than described by Pessagno (ray length $180 \mu$ vs. $324 \mu$ ) and broken so that a brachiopyle was not detected; patagium poorly developed or absent. It is the presence of other specific features (nodes, tabulas, etc.) which warrant the placing of specimens in this species.

This chapter: Plate 9, Figure 12; Tables 3, 5.

Cretaceous.

Haliomma erinaceum Haeckel, 1862

Renz, in press.

This chapter: Plate 14, Figure 11; Tables 4, 7.

Quaternary.

Haliomma minor Campbell and Clark, 1944b

Campbell and Clark, 1944b, p. 11, pl. 4, fig. 3.

Dimensions: ( 2 specimens) diameter cortical shell $90 \mu$; diameter medullary shell $34 \mu$; spine $48 \mu$.

This chapter: Plate 9, Figure 8; Table 5.

Cretaceous.

Haliomma sp. ef. $\underline{H}$. glisifra Renz, in press

Renz, in press.

Remarks: Specimens found in Leg 27 material tend to be more robust with shorter spines than the type species from the central Pacific.

This chapter: Plate 14, Figure 12; Tables 4, 6, 7, 8 .

Quaternary.

Heliodiscus asteriscus Haeckel, 1887

Renz, in press.

This chapter: Plate 13, Figure 11; Tables 2, 4, 6, 7, 8 .

Quaternary.

Heliodiscus echiniscus Haeckel, 1887

Nigrini, 1967, p. 34, pl. 3, fig. 2a, b.

This chapter: Plate 13, Figure 1; Table 7.

Quaternary.

?Hemicryptocapsa pseudopilula Tan Sin Hok, 1927

Tan Sin Hok, 1927, p. 51, pl. 9, fig. 69.

Dimensions: ( 3 specimens) width abdomen $135-144 \mu$; width thorax

33-45 $\mu$; length thorax $18-27 \mu$; width cephalis $15-18 \mu$; pore wall $12-15 \mu$.

Remarks: This species is slightly larger than described by Tan Sin Hok.

This chapter: Plate 5, Figures 10-13; Plate 12, Figure 5; Tables 1, 3,

5; Section 3.

Cretaceous.

Hexalonche sp.

Description: Medullary shell small with regular, hexagonal pores; cortical shell large with large rounded pores of various sizes; six stout primary spines; numerous small secondary spines.

Dimensions: (2 specimens) diameter medullary shell $\overline{48} \mu$; diameter

cortical shell $\Gamma T_{\mu}$; primary spine $\Gamma_{20} \mu$; secondary spine $\overline{9}_{\mu}$.

This chapter: Plate 14, Figure 13, Tables 2, 4, 6, 7.

Quaternary.

Hexapyle dodecantha Haeckel, 1887

Renz, in press.

This chapter: Plate 13, Figure 7; Tables 4, 6, 8 .

Quaternary.

incerti sedis

Dimensions: (2 specimens) width $80 \mu$; height $96 \mu$.

Remarks: Probably a cross-section view of the central area of a disc.

This chapter: Plate 9, Figure 10; Table 5.

Cretaceous.

Lamprocyclas maritalis Haeckel, 1887

Renz, in press.

This chapter: Plate 19, Figure 21; Tables 2, 4, 6, 7, 8; Section 2c. Quaternary.

Lamprocyrtis haysi Kling, 1973

Kling, 1973, p. 82 , pl. 5 , fig. 15,16 ; pl. 15, fig. 1-3.

This chapter: Plate 19, Figure 25; Tables 2, 4, 6, 7.

Quaternary.

Lamprocyrtis heteroporus (Hays, 1965)

Kling, 1973, p. 83, pl. 5 , fig. 19-21; pl. 15, fig. 6.

This chapter: Plate 19, Figure 25; Tables 2, 4, 6, 7.

Quaternary.

Lampromitra parabolica Popofsky, 1913

Renz, in press.
This chapter: Plate 16, Figure 9; Tables 4, 6, 7.

Quaternary.

Lamprospyris hookeri Haeckel, 1887

Renz, in press.

This chapter: Plate 19, Figure 30; Tables 4, 6, 7 .

Quaternary.

Lamprotripus butschlii (Haeckel, 1887)

Petrushevskaya, 1971b, fig. 50.1.

Remarks: Rib extensions are much reduced.

This chapter: Plate 18, Figure 23; Tables 4, 6, 7.

Quaternary.

Larcospira quadrangula Haeckel, 1887

Renz, in press.

This chapter: Plate 13, Figure 2; Tables 4, 6, 7, 8 .

Quaternary.

Liriospyris costata (Haeckel, 1887)

Nigrini, 1967, p. 45, pl. 5, fig. 4.

Remarks: The generic assignment of this species has been altered to

follow the revisions by Goll, 1968 .

This chapter: Plate 19, Figure 2; Tables 4, 6, 7.

Quaternary.

Liriospyris reticulata (Ehrenberg, 1872a)

Goll, 1968, p. 1429, pl. 176, fig. 9, 11, 13.

This chapter: Plate 19, Figure 1; Tables 2, 4, 6, 7.

Quaternary.

Liriospyris sp. 1

Dimensions: ( 2 specimens) height $108 \mu$; width $108 \mu$.

This chapter: Plate 19, Figure 3; Table 7.

Quaternary.

Liriospyris sp. 2

This chapter: Plate 19, Figure 4; Tables 4, 7.

Quaternary.

Litharachnium tentorium Haeckel, 1862

Renz, in press.

Remarks: Specimens broken.

This chapter: Plate 17, Figure 19; Tables 4, 6, 7

Quaternary.

Lithocampe chenodes n. sp.

Description: Cephalis spherical with large thick offset apical spine or stout corona-shaped spine; thorax and upper abdominal segments cylindrical numbering six to eight; lower abdominal segments becoming broader and forming a bulbous section which tapers toward the end; total number of segments 14-18; numerous stout spines protrude from this section; pores small, rounded and close set between which very irregular interstitial network of ridges can be seen.

Dimensions: ( 3 specimens) total height $327-372 \mu$; maximum width 150-180 $\mu$; apical spine $33-54 \mu$; abdominal spines $45-67 \mu$.

Remarks: This species differs from $L$. sp. aff. $L$. mediodilatata $\mathrm{R}$ üst, 1885 , as described by Moore, 1973, in the characteristics of the apical spine and abdominal spines; the greater number of segments; generally small size and proportions; and in the very characteristic irregular network of ridges. The specific name is Greek for goose-like.

Type locality: $12^{\circ} 56.83^{\prime} \mathrm{S}, 117^{\circ} 53.56^{\prime} \mathrm{E}$, DSDP Leg 27 , Sample 261 12-2, top.

This chapter: Plate 7, Figure 30; Plate 12, Figure 14 a-d; Tables 3, 5; Sections $2 \mathrm{~b}, \mathrm{c}, 3$.

Cretaceous.

?Lithocampe pseudochrysalis var. $\alpha$ Tan Sin Hok, 1927

Tan Sin Hok, 1927, p. 64, pl. 13, fig. 108.

Remarks: The specific features of the pore wall were obscured; all

other characteristics agree with Tan Sin Hok's description.

This chapter: Plate 11, Figure 31; Table 5.

Cretaceous.

Lithocampe sp.

Nigrini, 1967 , p. 87 , pl. 8 , fig. 6 a, b.

This chapter: Plate 16, Figure 4; Tables 2, 4, 6, 8.

Quaternary.

Lithocyclia? sp. A

Moore, 1973, p. 824, pl. 18, fig. 6.

Dimensions: ( 2 specimens) diameter central rings 63-75 $\mu$; diameter disc $240-255 \mu$.

Remarks: This species appears to be identical with that figured by

Moore, 1973, but its assignment to the genus Lithocyclia is in error.

This chapter: Plate 4, Figures 6, 7; Plate 10, Figure 16; Table 5; Section 3 .

Cretaceous. 
Lithomelissa monoceras Popofsky, 1913

Renz, in press.

This chapter: Plate 18, Figure 14; Tables 4, 6, 7.

Quaternary.

Lithomitra lineata group (Ehrenberg, 1838)

Renz, in press.

This chapter: Plate 17, Figure 12a, b; Tables 4, 7.

Quaternary.

Lithomitra pseudopinguis Tan Sin Hok, 1927

Tan Sin Hok, 1927, p. 57, pl. 10, fig. 86.

Description: Cephalis subspherical without an apical horn; thoracic and abdominal segments rounded getting progressively larger; segments vary from three to five; pores angular, irregular, closely spaced.

Dimensions: (3 specimens) total height $114-207 \mu$; maximum width $90 \mu$.

Remarks: Tetracapsa zinckenii Rüst, 1885 , may be conspecific, but the figure is so stylized and the description so brief that no decision is possible until topotypic material is examined. If at that time the species of Tan Sin Hok is found to be a new species, its generic assignment should be revised since it has little in common with the type species $L$. pachyderma Ehrenberg, 1873 (probably an Artostrobiid).

This chapter: Plate 7, Figures 5-7; Plate 11, Figure 30; Tables 1, 3, 5.

Cretaceous.

Lithopera bacca Ehrenberg, 1872a

Renz, in press.

This chapter: Plate 16, Figure 28; Tables 2, 4, 6, 7; Section 2e. Quaternary.

Lithopilium sphaerocephalum Popofsky, 1913

Renz, in press.

This chapter: Plate 16, Figure 22; Tables 4, 6, 7.

Quaternary.

?Lithostrobus erectus Tan Sin Hok, 1927

Tan Sin Hok, 1927 , p. 53, pl. 10, fig. 16.

Description: Cephalis subspherical, enveloped in a very stout apical

horn; segmentation slight; pores small, very irregular, giving an almost spongy appearance.

Dimensions: ( 2 specimens) total length $261 \mu$; maximum width 99 -

$135 \mu$; apical spine $69 \mu$.

Remarks: Number of segments varies from five to eight; apical spine is longer than described by Tan Sin Hok $(69 \mu$ vs. $29 \mu)$; but similar specimens were found in the material from Rotti, Site 149.

This chapter: Plate 7, Figures 12, 13; Plate 11, Figures 19a, b; Table 5.

Cretaceous.

Lithostrobus hexagonalis Haeckel, 1887

Renz, in press.

This chapter: Plate 16, Figure 19; Tables 4, 6, 7.

Quaternary.

Lophophaena cylindrica (Cleve, 1900)

Renz, in press.

This chapter: Plate 18, Figure 6; Tables 2, 4, 6, 7.

Quaternary.

Lophophaena sp.

Description: Three-lobed cephalis; collar constriction slight; third segment never complete; pores medium sized, irregularly rounded, moderately close-set.

Dimensions: (6 specimens); length $87-105 \mu$; maximum width 69 -

$75 \mu$; length cephalis $38 \mu$; length third segment $27 \mu$.

This chapter: Plate 12, Figure 9; Tables 1, 5.

Cretaceous.

Lophophaenoma sp. aff. L. witjazii Petrushevskaya, 1971b

Renz, in press.

This chapter: Plate 18; Figure 13; Tables 2, 4, 6, 7 .

Quaternary.

Lophophaeniid sp. A

Description: Three-lobed cephalis with three-bladed apical spine; three rather short legs begin at the collar constriction and emerge at the bottom of the thorax, pylome constricted; pores on cephalis and thorax, large, irregular, and close-set.

Dimensions: (1 specimen) total length $108 \mu$; width $78 \mu$; apical spine

$15 \mu$; leg $25 \mu$; pylome diameter $33 \mu$.

This chapter: Plate 12, Figure 11; Table 5.

Cretaceous.
Lophophaeniid sp. B

Description: Cephalis divided into four chambers by septa; three short wings originate from collar stricture and extend through the thorax wall; external surface appears rough; pores small, irregular, widely spaced.

Dimensions: ( 1 specimen) total length $102 \mu$; maximum width $72 \mu$; length cephalis $33 \mu$.

This chapter: Plate 12, Figure 10; Table 5.

Cretaceous.

Lophophaeniid sp. C.

Description: Large, simple cephalis with three to four short apical spines; collar stricture moderately strong; subsequent strictures weak; thorax subconical; abdomen and postabdominal segments cylindrical; pores small, very irregular, close-set, distributed over entire shell.

Dimensions: (1 specimen) length (5 segments) $141 \mu$; width $72 \mu$; cephalis length $45 \mu$; thorax length $30 \mu$; length of abdominal segment $21 \mu$.

This chapter: Plate 12, Figure 12; Table 5.

Cretaceous.

Myelastrinae subfamily group

Renz, in press.

This chapter: Plate 15, Figure 6; Table 7.

Quaternary.

Nassellarian gen. and sp. indet.

Dimensions: ( 2 specimens) height $\overline{150} \mu$; width $\overline{86} \mu$; spine $\overline{45} \mu$.

This chapter: Plate 16, Figure 26; Table 7.

Quaternary.

Nephrospyris renilla Haeckel, 1887

Renz, in press.

This chapter: Plate 19, Figure 6; Tables 4, 6, 7.

Quaternary.

Ommatartus tetrathalamus (Haeckel, 1887)

Renz, in press.

This chapter: Plate 14, Figure 5; Tables 2, 4, 6, 7, 8; Sections 2b-d. Quaternary.

Otosphaera polymorpha Haeckel, 1887

Nigrini, 1967, p. 23 , pl. 1, fig. 8.

This chapter: Plate 13, Figure 15; Tables 4, 6, 7, 8 .

Quaternary.

Patulibracchium petroleumensis Pessagno, 1971

Pessagno, 1971, p. 37, pl. 11, fig. 2-5.

Remarks: The presence of a cylindrical bracchiopyle in these

specimens is vague; majority of specimens without patagium.

This chapter: Plate 9, Figure 2; Tables 3, 5; Section 3.

Cretaceous.

Peromelissa phalacra (Haeckel, 1887), emend. Petrushevskaya, 1971b

Petrushevskaya, 1971b, p. 131, pl. 72, fig. XIV-XVII.

This chapter: Plate 18, Figure 7; Tables 2, 4, 7.

Quaternary.

Polysolenia spp.

Nigrini, 1967 , p. 13, pl. 1, fig. 1, 2, 3a, b.

Remarks: In this study, the species $P$. spinosa, $P$. flammabunda, and

$P$. lappacea were recognized and treated as a group.

This chapter: Plate 13, Figure 4; Tables 2, 4, 6, 7, 8 .

Quaternary.

Porodiscus delicatulus (Lipman, 1952)

Petrushevskaya and Kozlova, 1972, p. 525, pl. 5, fig. 9.

Dimensions: ( 2 specimens) diameter $174 \mu$; number of spiral whorls

7 , length of spines $24-39 \mu$.

Remarks: The rings discussed by Petrushevskaya and Kozlova are

joined in spiral whorls in all specimens observed.

This chapter: Plate 9, Figure 14; Tables 3, 5.

Cretaceous.

Porodiscus microporous (Stöhr, 1880)

Renz, in press.

This chapter: Plate 15, Figure 16; Tables 4, 6, 7,8.

Quaternary.

Porodiscus sp. aff. P. delicatulus (Lipman, 1952)

Petrushevskaya and Kozlova, 1972, p. 525, pl. 5, fig. 9.

Dimensions: ( 2 specimens) diameter $166 \mu$, number of spiral whorls

6 , number of spines 7-8.

Remarks: These specimens differ from $P$. delicatulus in having

thicker and generally fewer spiral whorls, and shorter spines. 
This chapter: Plate 9, Figure 7; Table 5.

Cretaceous.

Pseudodictyophimus gracilipes (Bailey, 1856) tetracanthus (Popofsky, 1913)

Petrushevskaya, 1971b, p. 95, pl. 49, fig. I-VII

This chapter: Plate 18, Figure 2; Tables 4, 7

Quaternary.

Psilomelissa calvata Haeckel, 1887

Renz, in press.

This chapter: Plate 18, Figure 8; Tables 4, 6, 7.

Quaternary.

Pterocanium bicorne Haeckel, 1887

Haeckel, 1887, p. 1332, pl. 73, fig. 15.

This chapter: Plate 17, Figure 6; Tables 4, 7.

Quaternary.

Pterocanium orcinum Haeckel, 1887

Haeckel, 1887, p. 1329, pl. 73, fig. 2.

This chapter: Plate 17, Figure 9; Table 4.

Quaternary.

Pterocanium praetextum (Ehrenberg, 1872a)

Renz, in press.

Remarks: No distinction was made between square- and round-

"shouldered" specimens.

This chapter: Plate 17, Figure 8; Tables 2, 4, 6, 7, 8; Sections 2b-d. Quaternary.

Pterocanium trilobum (Haeckel, 1860)

Renz, in press.

This chapter: Plate 17, Figure 7; Tables 2, 4, 6, 7, 8; Sections 2b-d. Quaternary.

?Pteropilium stratiotes Haeckel, 1887

Haeckel, 1887, p. 1326 , pl. 70, fig. 9.

Remarks: Lattice work never complete between wings and apical horn.

This chapter: Plate 16, Figure 27; Tables 4, 6, 7.

Quaternary.

Pyloniidae family group

Renz, in press.

This chapter: Plate 13, Figures 12, 13; Tables 2, 4, 6, 7, 8; Sections 2b-d.

Quaternary.

Rhopalodictyum sp.

Description: Three-armed Spongodiscid without concentric rings; arms with flared tips and three short terminal spines.

Dimensions: (2 specimens) arm length $135-150 \mu$; width at tip 99$117 \mu$.

This chapter: Plate 3, Figures 10, 11; Plate 10, Figure 2; Tables 3, 5. Cretaceous.

Rhopalastrum sp. 1

Description: Three-armed Spongodiscid; faint concentric rings noticeable in central area (approx. 4); arms thin, becoming bulbous only at tips; usually without patagium.

Dimensions: ( 3 specimens) diameter of central area $\overline{58} \mu$; arm length

141-207 $\mu$; arm width $\overline{30} \mu$; tip width $\overline{93} \mu$.

This chapter: Plate 15, Figure 2; Tables 2, 4, 6, 7, 8.

Quaternary.

Rhopalastrum sp. 2

Description: Three-armed spongodiscid; central area consisting of approximately five faint concentric rings; arms narrow but increasing in width to bulbous tips; patagium usually present, extending between tips but not enclosing them.

Dimensions: (3 specimens) diameter of central area $\overline{90} \mu$; arm length

$\overline{167} \mu$; arm tip width $\overline{129} \mu$.

This chapter: Plate 15, Figure 3; Tables 2, 4, 6, 7, 8 .

Quaternary.

Saturnalis circularis Haeckel, 1887

Nigrini, 1967 , p. 25 , pl. 1, fig. 9 .

This chapter: Plate 14, Figure 3; Tables 2, 4, 6, 7.

Quaternary.

Sethoconus myxobrachia Strelkov and Reshetnyak, 1959

Renz, in press.

This chapter: Plate 16, Figure 5; Table 7.

Quaternary.

Sethophormis pentalactis Haeckel, 1887

Renz, in press.
This chapter: Plate 18, Figures 18a, b; Tables 4, 7.

Quaternary.

Sethophormis rotula Haeckel, 1887

Renz, in press.

This chapter: Plate 18, Figure 17; Table 7.

Quaternary.

Sethophormis sp. aff. S. pentalactis Haeckel, 1887

Haeckel, 1887 , p. 1244 , pl. 56, fig. 5.

Remarks: This species has the same structure and dimensions as $S$. pentalactis but differs in the pore structure. Here the pores become progressively larger reaching a maximum at the margin which consists of a row of robust terminal spines.

This chapter: Plate 18, Figure 22; Table 7.

Quaternary.

Sethophormis sp.

Remarks: Only broken specimens observed, no cephalis. Four major radii, 16 interradii.

This chapter: Plate 18, Figure 21; Tables 4, 6, 7.

Quaternary.

Siphocampe corbula (Harting, 1863)

Renz, in press.

This chapter: Plate 17, Figure 14; Tables 4, 6, 7, 8 .

Quaternary.

Siphonosphaera polysiphonia Haeckel, 1887

Nigrini, 1967, p. 18, pl. 1, fig. 4a, b.

Remarks: Siphons irregularly curved in most specimens.

This chapter: Plate 13, Figure 9; Tables 2, 4, 6, 7, 8 .

Quaternary.

Siphonosphaera sp. A

Riedel, 1971, p. 651,'generic definition.

This chapter: Plate 13, Figure 10; Tables 4, 6, 7 .

Quaternary.

Siphonosphaera sp. B

Riedel and Sanfilippo, 1971, pl. 1c, fig. 3, 4.

This chapter Plate 13, Figure 3; Tables 4, 7 .

Quaternary.

Sphaerostylus lanceola (Parona, 1890

Foreman, 1973, pl. 1, fig. 7, 11.

Stylaractus ovatus Hinde in Moore, 1973.

This chapter: Plate 2, Figures 13-16; Plate 9, Figure 19; Tables 3, 5;

Section 3.

Cretaceous.

Spirocyrtis scalaris Haeckel, 1887

Renz, in press.

This chapter: Plate 17, Figure 16; Tables 4, 6, 7.

Quaternary.

Spirocyrtis sp. aff. S. scalaris Haeckel, 1887

Riedel and Sanfilippo, 1971, pl. 1G, fig. 19.

This chapter: Plate 17, Figure 15; Tables 2, 4, 6, 7.

Quaternary.

Spongaster tetras Ehrenberg, 1860

Nigrini, 1967, p. 41 , pl. 5, fig. 1a, b.

This chapter: Plate 15, Figure 8; Tables 2, 4, 6, 7, 8; Sections 2b, c.

Quaternary.

Spongobrachium sp. aff. S. ellipticum Haeckel, 1862

Renz, in press

This chapter: Plate 15, Figure 10; Tables 4, 7.

Quaternary.

Spongocore diplocylindrica Haeckel, 1887

Renz, in press.

This chapter: Plate 15, Figure 4; Tables 4, 6, 7 .

Quaternary.

Spongocyclia lanigera n. sp.

Description: A finely spongy disc with a spiral center; margin without spines.

Dimensions: (3 specimens) diameter spiral $114 \mu$; diameter disc $294 \mu$.

Remarks: This species differs from $S$. trachodes in the fine nature of its spongy shell; it appears quite similar to the Spongodiscid figured by Petrushevskaya and Kozlova, 1972, p. 529, pl. 5, fig. 13. The species name is Latin meaning fleecy.

Type locality: $12^{\circ} 56.85^{\prime} \mathrm{S}, 117^{\circ} 53.56^{\prime} \mathrm{E}$, DSDP Leg 27 , Sample 261 15 , CC.

This chapter: Plate 4, Figure 5; Plate 10, Figures 7a, b; Tables 3, 5; Sections 3, 4 . 
Cretaceous.

Spongocyclia trachodes n. sp.

Description: A roughly spongy disc with a large spiral central area; margin of disc with short spines.

Dimensions: (2 specimens) diameter of spiral $\overline{150} \mu$; diameter disc $210 \mu$.

Remarks: This species differs from Spongocyclia cycloides Haeckel, 1860 (type species) by its greater proportion of spongy material around the central spiral and from $S$. lanigera by the rough texture of this spongy material. The species name trachodes is Greek for "of a rough nature."

Type locality: $12^{\circ} 56.83^{\prime} \mathrm{S}, 117^{\circ} 53.56^{\prime} \mathrm{E}$, DSDP Leg 27 , Sample 26115-1, $9-11 \mathrm{~cm}$.

This chapter: Plate 4, Figures 1-4; Plate 10, Figure 13; Tables 3, 5; Section 3.

Cretaceous.

Spongodiscus sp. cf. S. americanus Kozlova, 1966

Sanfilippo and Riedel, 1973, p. 524, pl. 28, fig. 9.

Dimensions: ( 3 specimens) diameter 201-255 $\mu$; diameter central rings $42-60 \mu$; width spongy girdle $30-60 \mu$; spine $15-21 \mu$.

Remarks: This smaller Cretaceous species is probably only super-

ficially similar to $S$. americanus of Cenozoic deposits.

This chapter: Plate 3, Figure 12; Plate 10, Figure 6; Table 5.

Cretaceous.

Spongodiscus sp. 1

Dimensions: (2 specimens) diameter disc $156-165 \mu$; spine $36 \mu$; number spines 12-15.

This chapter: Plate 10, Figure 17; Table 5.

Cretaceous.

Spongodiscus sp. 2

This chapter: Plate 10, Figure 22; Table 5.

Cretaceous.

Spongodiscus sp. 3

Dimensions: (3 specimens) diameter 255-291 $\mu$.

This chapter: Plate 15, Figure 11; Tables 4, 6, 7.

Quaternary.

Spongodiscid 1 gen. and sp. indet.

Description: Four-armed spongodiscid; arms stubby, tipped with stout three-bladed spines.

Dimensions: ( 3 specimens) shell diameter $180-231 \mu$; spine $60210 \mu$.

This chapter: Plate 3, Figure 9; Plate 10, Figure 4; Tables 3, 5.

Cretaceous.

Spongodiscid 2 gen. and sp. indet.

Description: Large spongy sphere with 17 or more spines project-

ing from the center into several planes.

Dimensions: ( 2 specimens) diameter total $330 \mu$; diameter first

medullary shell $12 \mu$; second medullary shell $30 \mu$.

This chapter: Plate 10, Figure 23; Table 5.

Cretaceous.

Spongodiscid 3 gen. and sp. indet.

Description: Finely spongy sphere with double medullary shells in the center and 30 or more projecting spines.

Dimensions: ( 2 specimens) diameter total $330 \mu$; diameter first

medullary shell $12 \mu$; second medullary shell $30 \mu$.

This chapter: Plate 10, Figure 23; Table 5.

Cretaceous.

Spongolena sp.

Renz, in press.

This chapter: Plate 15, Figure 5; Tables 2, 4, 6, 7, 8.

Quaternary.

Spongoliva ellipsoides Popofsky, 1912

Renz, in press.

Remarks: Note the similarity between the medullary shell of $S$.

ellipsoides and $O$. tetrathalamus.

This chapter: Plate 14, Figure 6; Table 7.

Quaternary.

?Spongolonche grandis Campbell and Clark, 1944b

Campbell and Clark, 1944b, p. 7, pl. 4, fig. 7.

Remarks: This matches the species of Campbell and Clark in all

features except it lacks the hooklets at the base of the spines.

This chapter: Plate 10, Figure 12; Tables 3, 5.

Cretaceous.

?Spongolonche sp. aff. S. grandis Campbell and Clark, 1944b

Campbell and Clark, 1944b, p. 7, pl. 4, fig. 7.
Remarks: This species differs from $S$. grandis by possessing terminal spines only half as long.

This chapter: Plate 10, Figure 11; Tables 3, 5.

Cretaceous.

Spongoprunum sp. aff. Cyphantus probus Rüst, 1888

Petrushevskaya and Kozlova, 1972, p. 529, pl. 4, fig. 6.

Remarks: No specimens with the elongated spongy central shell were observed.

This chapter: Plate 2, Figures 19-22; Plate 10, Figure 19; Tables 3, 5.

Cretaceous.

Spongoprunum diversispina Squinabol, 1904

Squinabol, 1904, p. 199, pl. 4, fig. 2.

Description: Spongodiscid with an egg-shaped spongy shell from which protrude two stout three-bladed spines at opposite poles, generally unequal in length.

Dimensions: ( 2 specimens) long axis of spongy shell $\overline{139} \mu$; short axis $\Pi 10 \mu$; length of spines $75 \mu$, $120 \mu$.

Remarks: This species is similar to Spongoprunum sp. aff. Cyphantus probus Rust but larger with a denser spongy shell.

This chapter: Plate 10, Figure 18; Tables 3, 5.

Cretaceous.

Spongopyle ecleptos n. sp.

Description: A fine spongy, rather small, disc with a flared pylome, no internal stricture, short spines surround the margin of the disc with larger spines around the pylome.

Dimensions: (6 specimens) total length (diameter including pylome)

147-165 $\mu$; width (diameter of disc) $90-108 \mu$.

Remarks: This species differs from $S$. insolita by possessing short spines around the margin of the disc and by lacking any internal structure. The specific name is Greek for "very fine."

Type locality: $12^{\circ} 56.83^{\prime} \mathrm{S}, 117^{\circ} 53.56^{\prime} \mathrm{E}$, DSDP Leg 27, Sample 26112-2, top.

This chapter: Plate 3, Figures 2-6; Plate 10, Figure 14; Tables 3, 5. Cretaceous.

Spongopyle galeata n. sp.

Description: A Targe dense spongy disc with flared pylome; no internal structure; margin of the disc and pylome often with robust spines.

Dimensions: (5 specimens) length (diameter including pylome) 150$195 \mu$; width (diameter of disc) $117-184 \mu$; spines $95-150 \mu$.

Remarks: $S$. galeata is most similar to $S$. ecleptos, but differs from it by possessing a larger dense spongy shell and many times, long stout spines. The specific name galeata is Latin for "helmeted."

Type locality: $12^{\circ} 56.83^{\prime} \mathrm{S}, 117^{\circ} 53.56^{\prime} \mathrm{E}$; DSDP Leg 27, Sample 26112-2, top.

This chapter: Plate 10, Figure 8; Table 5.

Cretaceous.

Spongopyle insolita Kozlova, 1966

Riedel and Sanfilippo, 1970, pl. 2, fig. 2.

Petrushevskaya and Kozlova, 1972, pl. 5, fig. 10

Description: Helmet-shaped spongodiscid with concentric rings or spiral whorls in the "upper" part and a spongy flared pylome in the lower part; generally without spines.

Dimensions: ( 3 specimens) length $153-186 \mu$; width maximum 102 $123 \mu$; diameter of rings or whorls $57-72 \mu$.

Remarks: Specimens generally contain only one flared pylome, but a few individuals have been observed with two, three, and four flares.

This chapter: Plate 3, Figures 7, 8; Plate 10, Figure 10; Table 5. Cretaceous.

Spongopyle stauromorphos n. sp.

Description: Large spongy disc with a central area of narrow (up to

7) concentric rings from which radiate five stout spines often penetrating the margin; smaller secondary spines are also found on the margin and on the flared pylome.

Dimensions: (4 specimens) total length (diameter with pylome) 210$246 \mu$; width (diameter disc) $158-166 \mu$; primary spines from margin 28 $60 \mu$.

Remarks: This species differs from $S$. insolita by having a larger shell with stout spines and many narrow concentric rings. The specific name is Greek for "cross-shaped."

Type locality: $12^{\circ} 56.83^{\prime} \mathrm{S}, 117^{\circ} 53.56^{\prime} \mathrm{E}$ : DSDP Leg 27 , Sample 261 9, CC.

This chapter: Plate 3, Figures 1a, b; Plate 10, Figure 9; Tables 3, 5. Cretaceous. 
Spongopyle trabeata n. sp.

Description: A fine spongy disc with slightly noticeable concentric rings in the central area from which radiate a small number of thin spines; these sometimes penetrate the margin; the most outstanding feature is a large elongated pylome.

Dimensions: (4 specimens) total length (diameter including pylome) 211-243 $\mu$; width (diameter disc) $120-132 \mu$.

Remarks: This species differs from other Spongopyle species by possessing a vague internal structure with central radiating spines and an extremely long pylome. The specific name trabeata is Latin for "robed."

Type locality: $12^{\circ} 56.83^{\prime} \mathrm{S}, 117^{\circ} 53.56^{\prime} \mathrm{E}$; DSDP Leg 27 , Sample 261 12-2, top.

This chapter: Plate 10, Figure 15; Table 5.

Cretaceous.

Spongosaturnalis sp. aff. Saturnalis lateralis group Campbell and Clark, 1944b

Campbell and Clark, 1944b, p. 6, pl. 1, figs. 7, 10, 11, 13, 15.

Description: Elliptical outer ring with five to seven spines on each half ring; terminal spines strong; shell small, spongy; attached to outer ring by two spines.

Dimensions: (4 specimens) internal ring long axis $\overline{225} \mu$, short axis $87 \mu$; ring thickness $\overline{12} \mu$; length terminal spines $\overline{51-72} \mu$; spongy shell diameter $30 \mu$.

Remarks: Members of this group have similar proportions and spongy shells, but differ in the geometry of the terminal spines. One type is a long simple spine centered between two lesser spines; one tends to be bow-shaped with a simple spine, and one has a solitary fish-hook spine. These characteristics may be species specific, but in the material from Site 261 specimens were so rare that they are treated here as a species group.

This chapter: Plate 2, Figures 1-4; Plate 9, Figure 15; Tables 3, 5. Cretaceous.

Spongosaturnalis sp. aff. Saturnalis polymorphus Squinabol, 1914

Squinabol, 1914, p. 293 , pl. 3, fig. 11, 12; pl. 5, fig. 2-7.

Description: Large, thick outer ring more circular than elliptical; shell small, spongy, attached to outer ring by two strong spines; generally five long spines on each half ring; no terminal spines.

Dimensions: ( 3 specimens) internal ring diameter 192-201 $\mu$; ring thickness $24-39 \mu$; spine length $135 \mu$; spongy shell diameter approximately $30 \mu$.

Remarks: This species is placed in the genus Spongosaturnalis because its shell is spongy rather than porous as in Saturnalis.

This chapter: Plate 2, Figure 5; Plate 9, Figure 22; Table 5. Cretaceous.

Spongosphaera streptacantha Haeckel, 1862

Renz, in press.

This chapter: Plate 14, Figure 16; Tables 4, 6, 7.

Quaternary.

Spongotripus sp. ef. Tripodictya triacummata Lipman, 1952

Lipman, 1952, p. 33, pl. 2, fig. 2.

Petrushevskaya and Kozlova, 1972, p. 528, pl. 21, fig. 2.

Dimensions: (2 specimens) diameter $\overline{138} \mu$, spine $\overline{165} \mu$.

This chapter: Plate 10, Figure 3; Tables 3, 5.

Cretaceous.

Spongotrochus multispinus Haeckel, 1887

Renz, in press.

This chapter: Plate 15, Figure 9; Tables 4, 6, 7, 8 .

Quaternary.

?Spongotrochus polygonatus Campbell and Clark, 1944b

Petrushevskaya and Kozlova, 1972, p. 528, pl. 4, fig. 9, 10.

Dimensions: (2 specimens) diameter $\overline{50} \mu$; spine $\frac{66}{66}$; number of spines 8.

Remarks: Campbell and Clark described a species with only six radial spines, while this species has eight and that of Petrushevskaya and Kozlova probably has more than six.

This chapter: Plate 10, Figure 5; Table 5.

Cretaceous.

?Squinabolella putahensis Pessagno, 1969

Pessagno, 1969, p. 418, pl. 33, fig. 9.

Dimensions: ( 2 specimens) diameter cephalis $42 \mu$; diameter thorax with skirt $135 \mu$.

Remarks: Some cephalic structure was present but generally specimens were very poorly preserved; specific identification based on characteristics of the thoracic skirt.
This chapter: Plate 11, Figure 14; Table 5.

Cretaceous.

Staurostylus italicus Rüst, 1898

Rüst, 1898, pl. 2, fig. 5.

Description: Rather small sphere with four stout three-bladed

spines. Pores irregularly hexagonal, close-set.

Dimensions; (2 specimens) diameter sphere $96 \mu$; spine length $196 \mu$; pore diameter $6 \mu$.

Remarks: All specimens had broken spines.

This chapter: Plate 9, Figure 18; Tables 3, 5.

Cretaceous.

Stichocampe bironec Renz, in press

Renz, in press.

This chapter: Plate 17, Figure 3; Table 7.

Quaternary.

?Stichocapsa procera Hinde, 1908

Hinde, 1908, p. 740 , pl. 10, fig. 2.

Description: Cephalis hemispherical often with two short apical horns; segments rounded to oval becoming progressively larger (6-9); constrictions almost total, tending to separate lower segments, no pore structure is detectable; pore wall finely spongy, thick, and smooth showing no signs of segmentation.

Dimensions: ( 4 specimens) total height $183-255 \mu$; maximum width $123-165 \mu$.

Remarks: This species differs from that described by Hinde in being somewhat smaller and lacking a definite pore structure. The specimens found in Leg 27 material may be later descendants of $S$. procera which was discovered in Jurassic rocks on Rotti.

This chapter: Plate 7, Figures 8-11; Plate 11, Figure 25; Table 5; Section 3 .

Cretaceous.

Stichocapsa pseudodecora Tan Sin Hok, 1927

Tan Sin Hok, 1927, p. 72, pl. 16, fig. 137.

This chapter: Plate 11, Figure 29; Table 5.

Cretaceous.

?Stichocapsa rutteni Tan Sin Hok, 1927

Tan Sin Hok, 1927 , p. 71, pl. 15, fig. 133.

Dimensions: ( 2 specimens) height without apical spine 138-147 $\mu$; length thorax $21 \mu$; length of bulbous segment $98 \mu$; apical spine $15 \mu$.

Remarks: This species is quite similar to the concept of Tan Sin

Hok, but differs by possessing one smaller abdominal segment rather than two.

This chapter: Plate 11, Figure 5, Tables 1, 3, 5.

Cretaceous.

Stichocapsa wichmanni Tan Sin Hok, 1927

Tan Sin Hok, 1927, p. 71, pl. 15, fig. 132.

Dimensions: ( 2 specimens) total length $186 \mu$; maximum width $138 \mu$.

This chapter: Plate 11, Figure 20; Table 5.

Cretaceous.

?Stichocapsa sp. aff. S. rutteni Tan Sin Hok, 1927

Tan Sin Hok, 1927, p. 71, pl. 15, fig. 133.

Remarks: This rare species is identical with ?S. rutteni except for an additional bulbous segment measuring $50-60 \mu$ long. These may well be one species.

This chapter: Plate 11, Figure 6; Tables 3, 5.

Cretaceous.

Stichomitra asymbatos Foreman, 1968

Foreman, 1968, p. 73, pl. 8, fig. 10a-c.

Dimensions: ( 2 specimens) total height $174-270 \mu$; maximum width

$111-153 \mu$; apical spine $24 \mu$; number of segments 3-6.

Remarks: A tapering terminal segment was not observed.

This chapter: Plate 11, Figure 21; Tables 1, 3, 5; Section 3.

Cretaceous.

?Stichomitra campi (Campbell and Clark, 1944b), emend. Foreman, 1968

Foreman, 1968 , p. 75 , pl. 8, fig. 3a-c.

Dimensions: ( 2 specimens) total height $\overline{108} \mu$; maximum width $\overline{72} \mu$; number of segments 4-5.

This chapter: Plate 11, Figure 16; Tables 3, 5.

Cretaceous.

Stichomitra cathara Foreman, 1968

Foreman, 1968, p. 73, pl. 8, fig. 9.

Dimensions: (2 specimens) total height $\overline{35} \mu$; maximum width $\overline{78} \mu$; number of segments 4 . 
Remarks: No complete specimens were observed.

This chapter: Plate 11, Figure 17; Table 5.

Cretaceous.

Stichopilium bicorne Haeckel, 1887

Renz, in press.

This chapter: Plate 16, Figure 24; Tables 4, 6, 7 .

Quaternary.

Stylatractus universus Hays, 1970

Dinkleman, 1973, p. 765, pl. 10, fig. 6, 7 .

This chapter: Plate 14, Figure 8; Tables 2, 4, 6, 7, 8 .

Quaternary.

Stylochlamydium sp. aff. S. venustum (Bailey, 1856)

Renz, in press.

Remarks: Shell larger and thicker with more rings than pictured by Renz.

This chapter: Plate 15, Figure 17; Tables 4,6, 7.

Quaternary.

Stylodictya sp. aff. S. multispina Haeckel, 1862

Renz, in press

Remarks: This species differs from that pictured in Renz in that the

spines seldom extend beyond the rim of the shell and the outermost oncentric ring appears incomplete as concave arcs.

This chapter: Plate 15, Figure 12; Tables 2, 4, 6, 7, 8; Section 2c.

Quaternary.

Stylosphaera pusilla Campbell and Clark, 1944b

Campbell and Clark, 1944b, p. 5, pl. 1, fig. 2, 4, 5.

Dimensions: ( 3 specimens) diameter cortical shell $\overline{77} \mu$; spine 69 $135 \mu$.

Remarks: In many specimens the terminal spines may appear rounded rather than three-bladed.

This chapter: Plate 2, Figures 17, 18; Plate 9, Figure 20; Table 5; Section 3.

Cretaceous.

Styptosphaera spongiacea Haeckel, 1887

Renz, in press.

This chapter: Plate 15, Figure 13; Tables 4, 6, 7 .

Quaternary.

Stylosphaera sp.

Dimensions: (2 specimens) diameter medullary shell 24-39 $\mu$; diameter cortical shell $63-75 \mu$; spine $84-96 \mu$.

This chapter: Plate 14, Figure 7; Tables 2, 4, 6, 7 .

Quaternary.

?Stylotrochus antiquus Campbell and Clark, 1944b

Campbell and Clark, 1944b, p. 19, pl. 4, fig. 8, 12.

Dimensions: (4 specimens) diameter $171-320 \mu$; spines $51-74 \mu$.

Remarks: Specimens are generally larger in diameter with longer

spines than described by Campbell and Clark.

This chapter: Plate 10, Figure 21; Tables 3, 5 .

Cretaceous.

Stylotrochus sp.

Campbell and Clark, 1944b, p. 20, pl. 5, fig. 6.

Description: Lenticular, rather small, spongy shell with six stout three-bladed spines (twisting slightly) protruding at strange angles.

Dimensions: (2 specimens) total height $\overline{108} \mu$; maximum width $\overline{72} \mu$; number of segments 4-5.

This chapter: Plate 10, Figure 1; Table 5.

Cretaceous.

Tessarastrum straussii Haeckel, 1887

Renz, in press.

Remarks: Cross arms rudimentary or completely lacking.

This chapter: Plate 15, Figure 15; Tables 4, 6.

Quaternary.

Tetracorethra tetracorethra (Haeckel, 1887), emend. Petrusherskaya, $1971 b$

Renz, in press.

Remarks: Apical spine always broken.

This chapter: Plate 19, Figure 18; Table 7.

Quaternary.

Theocalyptra sp.

Renz, in press.

This chapter: Plate 16, Figure 21; Tables 2, 4, 6, 7.

Quaternary.

?Theocapsa elata Tan Sin Hok, 1927

Tan Sin Hok, 1927 , p. 47, pl. 8, fig. 52.

Dimensions: ( 3 specimens) total height $177-186 \mu$; maximum width

$135-150 \mu$; wall thickness $15 \mu$; thorax length $38 \mu$.
Remarks: This species differs from Tan Sin Hok's description in lacking an apical horn and being generally broader.

This chapter: Plate 6, Figure 20; Plate 11, Figure 8; Tables 1, 5. Cretaceous.

Theoconus hertwigi (Haeckel, 1887)

Renz, in press.

Remarks: All specimens had ribs.

This chapter: Plate 19, Figure 16; Tables 4, 6, 7.

Quaternary.

Theoconus junonis Haeckel, 1887

Renz, in press.

This chapter: Plate 19, Figure 27; Tables 2, 4, 6, 7, 8 .

Quaternary.

Theocorys veneris Haeckel, 1887

Renz, in press.

Remarks: Wings may or may not be present.

This chapter: Plate 16, Figure 18; Tables 4, 6, 7.

Quaternary.

Theocorys sp. aff. T. antiqua Squinabol, 1903

Squinabol, 1903, p. 135, pl. 8, fig. 25 .

Dimensions: (5 specimens) height $123-159 \mu$; maximum width 78 $108 \mu$.

Remarks: This species, though very similar to the description by

Squinabol, differs from it in being wider and longer with pores more closely set.

This chapter: Plate 6, Figures 4-7; Plate 11, Figure 4; Tables 1, 3, 5. Cretaceous.

Theocorythium trachelium (Ehrenberg, 1872a)

Renz, in press.

This chapter: Plate 19, Figure 28; Tables 2, 4, 6, 7, 8.

Quaternary.

Theophormis callipilium Haeckel, 1887

Haeckel, 1887 , p. 1367 , pl. 70 , fig. 1

Remarks: Four major radii; 8-12 interradii; none penetrate outer

"skirt."

This chapter: Plate 18, Figure 24; Tables 4, 7.

Quaternary.

Theopilium pyramidale Popofsky, 1913

Renz, in press.

This chapter: Plate 16, Figure 17; Tables 4, 6, 7.

Quaternary.

Tholodiscus fresnoensis (Foreman, 1968)

Petrushevskaya and Kozlova, 1972, p. 525, pl. 5, fig. 1.

This chapter: Plate 9, Figure 9; Table 5.

Quaternary.

Tholospyris scaphipes (Haeckel, 1887)

Goll, 1969, p. 328, pl. 58, fig. $1-8,13,14$

Dimensions: ( 3 specimens) height $60 \mu$; width $71 \mu$; feet $48 \mu$.

This chapter: Plate 19, Figure 13; Tables 4, 6, 7.

Quaternary.

Tholospyris tripodiscus Haeckel, 1887

Renz, in press.

This chapter: Plate 19, Figure 29; Tables 4, 6, 7.

Quaternary.

Tholospyris sp.

Renz, in press.

This chapter: Plate 19, Figure 12; Tables 2, 4, 6, 7.

Quaternary.

?Tricolocapsa parvipora Tan Sin Hok, 1927

Tan Sin Hok, 1927, p. 48, pl. 9, fig. 59, 60.

1968 ? Solenotryma sp. cf. $S$. dacryodes Foreman, p. 33, pl. 4, fig. 7.

Description: Cephalis subspherical without apical horn; lumbar stricture moderate; position highly variable; later abdominal segments appear to be appendages rather than a fundamental part of the skeleton; pores, small, sparse, irregular.

Dimensions: (4 specimens) total height $96 \mu$ (3 segments) $-198 \mu$ (7 segments); maximum width $75-87 \mu$.

Remarks: Specimens with numerous postabdominal segments are rare. More common are simple three-segmented specimens which have not developed the appendage of segments, as pictured in Tan Sin Hok. In this study, these are treated as one species. T. parvipora is similar to Solenotryma dacryodes of Foreman in many features, but has consistently lacked a terminal tube.

This chapter: Plate 6, Figures 8-12; Plate 11, Figure 3; Tables 1, 3, 5.

Cretaceous

?Tripocalpis ellyae Tan Sin Hok, 1927

Tan Sin Hok, 1927, p. 38, pl. 7, fig. 18. 
Dimensions: ( 2 specimens) length without apical horn $165 \mu$; maximum width $102 \mu$; apical horn $66 \mu$; length of feet $75 \mu$.

Remarks: This species differs from that described by Tan Sin Hok, 1927 , in having an almost spongy porous network enveloping the cephalis. It also differs from $D$. gracilis in the same manner as well as possessing a distinctively different apical horn which is more spear shaped than crown shaped.

This chapter: Plate 5, Figures 18, 19; Plate 11, Figure 10; Table 5. Cretaceous.

?Tripodictya elegantissima Vinassa, 1899

Vinassa, 1899, p. 231, pl. 2, fig. 3.

Dimensions: ( 3 specimens) diameter disc $108 \mu$; spines $165 \mu$.

Remarks: This species is similar to the description of Vinassa, but

differs from it by possessing three very long three-bladed spines.

This chapter: Plate 1, Figures 13-15; Plate 9, Figure 5; Table 5. Cretaceous.

Tympanidium foliosum Haeckel, 1887

Renz, in press.

This chapter: Plate 19, Figure 5; Tables 4, 6, 7.

Quaternary.

Udan undulata Renz, in press.

Renz, in press.

This chapter: Plate 16, Figure 14; Table 4.

Quaternary.

Verticillata hexacantha Popofsky, 1913

Renz, in press.

This chapter: Plate 18, Figure 1; Tables 4, 6, 7.

Quaternary.

Xiphodictya sp. cf. $X$ ovalis Rüst, 1898

Rüst, 1898 , p. 23, pl. 7 , fig. 6 .

Remarks: This species is only superficially similar to the description of Rüst having a central shell with concentric rings and two strong opposing spines and being of like proportions and size.

This chapter: Plate 9, Figure 13; Table 5.

Cretaceous.

Xiphosphaera tesseractis Dreyer, 1913

Renz, in press.

Remarks: Spines always broken.

This chapter: Plate 14, Figure 15; Tables 4, 7.

Quaternary.

Xiphosphaera umbilicata Rüst, 1898

1927 Xiphosphaera tuberosa Tan Sin Hok

in press Acaeniotyle umbilicata (Rust) in Foreman

Rust, 1898 , p. 7, pl. 1, fig. 9.

Dimensions: ( 4 specimens) diameter $120-138 \mu$; spine length 96-

$135 \mu$; pore wall $12-15 \mu$.

Remarks: As with Foreman, the internal structure is vague. The

polar spines are not as long as Rust described and never curved. No large-shelled specimens were observed.

This chapter: Plate 2, Figures 9-12; Plate 9, Figure 21; Tables 1, 3,

Cretaceous.

Zygocircus capulosus Popofsky, 1913

Petrushevskaya, 1971 b, p. 281, pl. 28, figs. VII, VIII.

This chapter: Plate 19, Figure 20; Tables 2, 4, 6, 7.

Quaternary.

Zygocircus sp. 1 aff. Z. capulosus Popofsky, 1913

Popofsky, 1913, p. 287, pl. 28, fig. 3.

This chapter: Plate 19, Figure 15; Table 7.

Quaternary.

Zygocircus sp. 2 aff. Z. capulosus Popofsky, 1913

Renz, in press.

This chapter: Plate 19, Figure 23; Tables 4, 6, 7 .

Quaternary.

Zygocircus sp. cf. Z. piscicaudatus Popofsky, 1913

Popofsky, 1913, p. 287, pl. 28, fig. 3.

This chapter: Plate 19, Figure 24; Tables 2, 4, 7, 8.

Quaternary.

\section{ACKNOWLEDGMENTS}

Financial support for this project was provided by the National Science Foundation through the Deep Sea Drilling Project. I would like to thank the Geology Department of the University of California, Los Angeles and especially Drs. Loeblich for the use of their facilities. A very special thanks to W. R. Riedel whose help and advice made this project possible at the beginning and influenced many of the conclusions at the end. Appreciation goes to Annika Sanfilippo for her time and thoughts and to Mary Hanger and Midge Neely for their time and typing.

\section{REFERENCES}

Aliev, K. S., 1961. Novye vidy semeistva Porodiscidae iz otlozhenii valanzhina severo-vostochnogo Azerbaidzhana: Dokl. AN Azerb. SSSR, t. 17, no. 7.

Bailey, J. W., 1856. Notice of microscopic forms found in the soundings of the Sea of Kamtschatka-with a plate: Am. J. Sci., v. 22 , p. 1-6.

Campbell, A. S. and Clark, B. L., 1944a. Miocene radiolarian faunas from Southern California: Geol. Soc. Am., Spec. Papers. 51.

1944b. Radiolaria from Upper Cretaceous of Middle California: Geol. Soc. Am. Spec. Papers, v. 57, p. i-viii, 161.

Cayeus, L., 1897. Contribution à l'étude micrographique des terrains sédimentaries 1. Etude de quelques dépots siliceux secondaries et tertiaires du Bassin de Paris et de la Belgique. 2. Craie du Bassin de Paris: Soc. Géol. Nord, Mém., v. 4 (2).

Cleve, P. T., 1900. Notes on some Atlantic plankton organisms: Handl. Kgl. Svenska Vetensk.-Akad., v. 34, p. $1-22$.

Deflandre, G., 1953. Radiolaires fossiles. In Traité de zoologie: Grassé, P. P. (Ed.), Paris (Masson), v. 1, p. 389436.

Dinkleman, M. G., 1973. Radiolarian stratigraphy: Leg 16, Deep Sea Drilling Project. In van Andel, T. H., Heath, G. R., et al., Initial Reports of the Deep Sea Drilling Project, Volume 14: Washington, (U.S. Government Printing Office), p. 747-813.

Dreyer, F., 1913. Die Polycystinen der Planton-Expedition: Ergebn. Plankton-Exped. Humboldt-Stiftung, v. 3, p. 1104.

Dumitrica, P., 1970. Cryptocephalic and cryptothoracic Nassellaria in some Mesozoic deposits of Romania: Rev. Roum. Geol., Geophys. Geogr. Ser. Geol., v. 14, p. 45-124.

Ehrenberg, C. G., 1838. Uber die Bildung der Kreidefelsen und des Kreidemergels durch unsichtbare Organismen: Abhandl. Akad. Wiss. Berlin, Jahrg. 1838, p. 59-147.

1844. Uber 2 neue Lager von Gebirgsmassen aus Infusorien als Meeres-Absatz in Nord-Amerika und eine Vergleichung derselben mit den organischen KreideBebilden in Europa und Afrika: Kgl. Preuss. Akad. Wiss. Berlin, Ber., Jahrg. 1844, p. 57-97.

1847. Uber die mikroskopischen kieselschaligen Polycystinen als mächtige Gebirgsmasse von Barbados: $\mathrm{Kgl}$. Preuss. Akad. Wiss. Berlin, Monatsber., Jahrg. 1847, p. 4060.

1854. Die systematische Characteristik der neuen mikroskopischen Organismen des tiefen Atlantischen Oceans: Kgl. Preuss. Akad. Wiss. Berlin, Monatsber., Jahrg. 1854 , p. $236-250$.

1860. Uber den Tief grund des stillen Oceans zwischen Californien und des Sandwich-Inseln: Kgl. Preuss. Akad. Wiss. Berlin, Monatsber., Jahrg. 1860, p. 819-833.

1872a. Mikrogeologischen Studien als Zusammenfassung seiner Beobachtungen des Kleinsten Lebens der Meeres-Tiefgründe aller Zonen und dessen geologischen Einfluss: Kgl. Preuss. Akad. Wiss. Berlin, Monatsber., Jahrg. 1872, p. 265-322.

1872b. Mikrogeologischen Studien über das Kleinste Leben der Meeres-Tiefgründe aller Zonen und dessen geologischen Einfluss: Abhandl. Kgl. Akad. Wiss. Berlin, Jahrg. 1872, p. 131-399.

1973. Grossere Felsproben des Polycystinen-Mergels 
Expedite: Geologische Verkenningstochten in CentraalBorneo (1893-1894): Leiden (Brill).

1908. Radiolaria from Triassic and other rocks of the Dutch East Indian Archipelago: Jaar. Mijn. Ned. OostIndië, Verhandl, v. 37, p. 1-42.

Kling, S. A., 1973. Radiolaria from the Eastern North Pacific, Deep Sea Drilling Project, Leg 18. In Kulm, L. D., von Huene, R., et al., Initial Reports of the Deep Sea Drilling Project, Volume 18: Washington (U.S. Government Printing Office), p. 617-671.

Kozlova, G. E. and Gorbovets, A. N., 1966. Radiolyarii verkhnemelovykh i verkhneeotsenovykh otlozhenii Zapadno-Sibirskoi Nizmennosti: Tr. Vses. Neft. Nauchn.Issled. Geol. Inst. (VNIGRI), v. 248.

Ling, H. Y., 1973. Radiolaria: Leg 19 of the Deep Sea Drilling Project. In Creager, J. S., Scholl, D. W., et al., Initial Reports of the Deep Sea Drilling Project, Volume 19: Washington (U.S. Government Printing Office), p. 777-797.

Lipman, R., 1952. Materialy morphographicheskomu izucheniyu radiolyarii verkhnemelovykh otlozhenii russkoi platformy: Paleontol. Strat. Vses. Nauchn-Issled. Geol. Inst., p. 24-51.

1960. Radiolaria. In Stratigrafiya i fauna melovykh otlozhenii zapadno-sibirskoi nizmennosti: Min. Geol. SSSR, Tr. Vses. Nauchn.-Issled. Geol. Inst. (VSEGEI), new ser. 29 , p. 124

Moore, T. C. Jr., 1973. Radiolaria from Leg 17 of the Deep Sea Drilling Project. In Winterer, E. L., Ewing, J. L., et al., Initial Reports of the Deep Sea Drilling Project, Volume 17: Washington (U.S. Government Printing Office), p. $797-$ 869.

Müller, J., 1858. Uber die Thalassicallen, Polycystinen und Acanthometran des Mittlemeeres: Kgl. Akad. Wiss. Berlin, Abhandl. Jahrg. 1858.

Nigrini, C. A., 1967. Radiolaria in pelagic sediments from the Indian and Atlantic Oceans: Scripps Inst. Oceanogr. Bull., v. 11, p. $1-125$.

1971. Radiolarian zones in the Quaternary of the equatorial Pacific Ocean. In The micropalaeontology of oceans: Funnel, B. M. and Riedel, W. R. (Eds.), Cambridge (Cambridge Univ. Press), p. 443-461.

Pantanelli, D., 1880 I diaspri della Toscana e i bro fossili: Atti R. Accad. Lincei, Rome, Mem. 8, p. 35-66.

Parona, C. F., 1890. Radiolarie nei noduli selciosi del calcare giurese di Cittiglio presso Laveno: Soc. Geol. Ital. Boll. 9, p. 1-46.

Pessagno, E. A., 1969. The Neosciadiocapsidae, a new family of upper Cretaceous Radiolaria: Am. Paleontol. Bull. 56.

1971. Jurassic and Cretaceous Hagiastridae from the Blake-Bahama Basin (Site 5A, JOIDES Leg 1) and the Great Valley sequence, California Coast Ranges: Am. Paleontol. Bull. 60, p. 1.

Petrushevskaya, M. G., 1965. Osobennosti Konstruktsii skeleta radiolyarii Botryoidae (otr. Nassellaria): T. Zool. Inst. (Akad. Nauk SSSR), v. 35, p. 79-118.

1971a. On the natural system of polycystine Radiolaria (Class Sarcodina): Plankt. Conf. Second Proc. Roma 1970, Farinacci, A. (Ed.), v. 2, p. 981-991.

1971b. Radiolyarii Mirovogo Okeana po materialam Sovetskikh ekspeditsii. In Bykhovshii, B. E. (Ed.) Issledovaniya Fauny Morei: Leningrad (Nauka), v. 9, p. 5294.

Petrushevskaya, M. G. and Kozlova, G. E., 1972. Radiolaria: Leg 14, Deep Sea Drilling Project. In Hayes, D. E., Pimm, A. C., et al., Initial Reports of the Deep Sea Drilling Project, Volume 14: Washington (U.S. Government Printing Office), p. 495-648.

Popofsky, A., 1908. Die Radiolarien der Antarktis (mit Ausnahme der Tripyleen), Deutsch. Sudpolar-Exped. 1901-
1903: vol. 10, (Zool. vol. 2), p. 183-305

1912. Die Sphaerellarien des Warmwassergebietes, Deutsche Sudpolar-Exped. 1901-1903: v. 13, (Zool., v. 5), p. $73-159$.

1913. Die Nassellarien des Warmwassergebietes, Deutsche Sudpolar-Exped. 1901-1903: v. 14, (Zool., v. 6), p. 217-416.

Principi, P. 1909. Contributo allo studio dei Radiolari Miocenici Italiani: Soc. Geol. Ital. Boll., v. 28, p. 1-22.

Renz, G. W., in press. The distribution and ecology of Radiolaria in the Central Pacific-plankton and surface sediments: Scripps Inst. Oceanogr. Bull., Univ. Calif. Press.

Riedel, W. R., 1967a. Class Actinopoda. Protozoa. In Harland, W. B. et al., The fossil record, A symposium with documentation: London (Geol. Soc., London), p. 291-298. 1967b. Some new families of Radiolaria: Geol. Soc. London, Proc. no. 1640, p. 148-149. 1971. Systematic classification of polycystine Radiolaria. In Funnel, B. M. and Riedel, W. R. (Eds.), The micropaleontology of oceans: Cambridge (Cambridge Univ. Press), p. 649-661.

Riedel, W. R. and Sanfilippo, A., 1970. Radiolaria, Leg 4. Deep Sea Drilling Project. In Bader, R. G., Gerard, R. O., et al., Initial Reports of the Deep Sea Drilling Project, Volume 4: Washington (U.S. Government Printing Office), p. 503 .

1971. Cenozoic Radiolaria from the western tropical Pacific, Leg 7. In Winterer, E. L., Riedel, W. R., et al., Initial Reports of the Deep Sea Drilling Project, Volume 7: Washington (U.S. Government Printing Office), p. 1529-1672.

Rüst, D., 1885. Beiträge zur Kenntniss der fossilen Radiolarien aus Gesteinen des Jura: Palaeontographica, v. 31, p. 273.

1888. Beiträge zur Kenntniss der fossilen Radiolarien aus Gesteinen der Kreide: Palaeontographica, v. 34, p. 181 1898. Neve Beitrage zur Kenntniss der fossilen Radiolarien aus Gesteinen des Jura und der Kreide: Palaeontographica, v. 45 , p. 1-67.

Sanfilippo, A. and Riedel, W. R., 1970. Post-Eocene "closed" theoperid radiolarians: Micropaleontology, v. 16, p. 446.

1973. Cenozoic Radiolaria (exclusive of Theoperids, Artostrobiids, and Amphipyndacids) from the Gulf of Mexico, Deep Sea Drilling Project, Leg 10. In Worzel, J. L., Bryant, W., et al., Initial Reports of the Deep Sea Drilling Project, Volume 10: Washington (U.S. Government Printing Office), p. 475-611.

Squinabol, S., 1903. Le Radiolarie dei noduli selciosi nella scaglia degli Euganei: Riv. Ital. Paleontol., p. 105.

1904. Radiolarie cretacee degli Euganei: Atti Mem. R. Accad. Sci. Lett. Arti Padova., v. 20, p. 171-244.

1914. Contributo alla conoscenza dei Radiolarii fossili del Veneto. Appendice: $\mathrm{Di}$ un genere di Radiolari caratteristico del Secondario: Geol. Univ. Padova, Mem. 2, p. 249.

Stöhr, E., 1880. Die Radiolarienfauna der Tripoli von Grotte. Provinz Girgenti in Sicilien: Palaeontographica, v. 2, p. 69. 124.

Strelkov, A. A. and Reshetnyak, V. V., 1959. Novaya zhiznennaya forma $\mathrm{u}$ radiolyarii, (A new life form in Radiolaria): Zool. Zhurn., v. 38, p. 355-361.

Tan Sin Hok, 1927. Over de samenstelling en hat onstaan van krijt-en mergelgesteenten van de Molukken: Jaar. Mijn. Ned.-Vost-Indië, Jahrg. 1926, Verhandl., pt. 3, p. 5-165.

Vinassa de Regny, P. E., 1899. I Radiolari delle ftaniti titoniane di Cárpena (Spezia): Palaeontogr. Ital., v. 4, p. 217 238.

Zittel, K. A., 1876. Ueber einige fossile Radiolarien aus der norddeutschen Kreide: Deut. Geol. Ges. Z., v. 28, p. 75. 
von Barbados: Kgl. Preuss. Akad. Wiss. Berlin, Monatsber., Jahrg. 1873, p. 213-263.

1875. Forsetzung der mikrogeologischen Studien als Gesammt-Uebersicht der mikroskopischen Pälaontologie gleichartig analysirter Gebirgsarten der Erde, mit specieller Rücksicht auf den Polycystinen-Mergel von Barbados: Preuss. Akad. Wiss. Berlin, Jahrg. 1875, p. 1-226.

Foreman, H. P., 1968. Upper Maestrichtian Radiolaria of California: Spec. Pap. Palaeontol. (Palaeontol. Assoc., London), No. 3.

1973. Radiolaria from Leg 20 of the Deep Sea Drilling Project. In Initial Reports of the Deep Sea Drilling Project, Volume 20: Washington (U.S. Government Printing Office), p. 249.

Goll, R. M., 1968. Classification and phylogeny of Cenozoic Trissocyclidae (Radiolaria) in the Pacific and Caribbean Basins. Part I: J. Paleontol., v. 42, p. 1409-1432.

1969. Classification and phylogeny of Cenozoic Trissocyclidae (Radiolaria) in the Pacific and Caribbean Basins. Part II: J. Paleontol., v. 43 , p. $322-339$. 1972. Leg 9 Synthesis, Radiolaria. In Hays, J. D. et al., Initial Reports of the Deep Sea Drilling Project, Volume 9: Washington (U.S. Government Printing Office), p. 9471058.

Haeckel, E., 1860. Fernere Abbildungen und Diagnosen neuer Gottungen und Arten von lebenden Radiolarien des Mittlemeeres: Kgl. Preuss. Akad. Wiss., Monatsber., Jahrg. 1860, p. $835-845$.

1862. Die Radiolarien (Rhizopoda Radiolaria), Eine Monographie: Berlin (Reimer).

1881. Entwurf eines Radiolarien-Systems auf Grund von Studien der Challenger-Radiolarien: Jena. Z. Med. Naturwiss., v. 15, p. 418-472.

1887. Report on the Radiolaria collected by H. M. S. Challenger during the years 1873-1876: Rept. Voyage Challenger, Zool., v. 18.

Haecker, V., 1908. Tiefsee-Radiolarien: Wiss. Ergebn. Deutschen Tiefsee Exped. (Valdivia), v. 14, p. 337-476.

Harting, P., 1863. Bijdrage tot de kennis der mikroskopische faune en flora van de Binde-zee: Verhindh. Koninkl. Akad. Wetensch. Asterdam, v. 10, p. 1-34.

Hays, J. D., 1965. Radiolaria and late Tertiary and Quaternary history of Antarctic seas: Antarctic Res. Ser. (Am. Geophys. Union), v. 5, p. 125-184.
1970. Stratigraphy and evolutionary trends of Radiolaria in North Pacific deep sea sediments: Geol. Soc. Am. Mem., v. 126, p. 185.

Hertwig, R., 1879. Der Organismus der Radiolarien: Jena (G. Fischer).

Hinde, G. J., 1900. Description of fossil Radiolaria from the rocks of Central Borneo. In Molengraaff, G.A.F., Borneo-

\section{PLATES}

The plates are of two types. First is a synchronopticon of Site 261, Cores 9-23 (Plates 1-8). Here each plate is divided horizontally into thirds, the top representing the Bathropyramis timorensis Assemblage; the middle, the Eucyrtis columbarius Assemblage; and the bottom, the Spongocyclia lanigera Assemblage. The location of the photo in each third, i.e., top, middle, or bottom, designates the core in which that specimen was found.

The key to these locations is as follows:

$$
\begin{aligned}
& \begin{array}{ll}
\text { top third: } & \text { Core } 9 \\
\text { middle third: } & \text { top-Core } 10
\end{array} \\
& \text { middle-Core } 12 \\
& \text { bottom-Core } 13 \\
& \text { bottom third: top-Core } 15 \\
& \text { middle-Core } 16 \\
& \text { bottom-Core } 23
\end{aligned}
$$

In those instances when it has not been possible to find a suitable specimen in a sample where its presence has been noted, a "+" has been entered at the appropriate level.

The next group of plates (9-19) is a systematic listing of the species discussed. Cretaceous and Quaternary species are grouped separately. All sample intervals are in centimeters. 
a. Synchronopticon

(Plates 1-8)

\section{PLATE 1 \\ (Magnification $\times 150$, unless otherwise indicated)}

Figures 1-6 Amphibrachium (?) hastatum n. sp.
1. 261-9, CC.
2. 261-10-1, 10-12.
3. 261-12, CC.
4. 261-13, CC.
5. 261-15-1, 9-11.
6. 261-23, CC.

Figures 7, 8 Hagiastrid 1 gen. and sp. indet.

7. 261-15-1,9-11.

8. 261-16-1, 101-103.

Figures 9, 10 Hagiastrid 2 gen. and sp. indet.
9. 261-12, CC.
10. 261-16-1, 101-103.

Figures 11, 12 Crucella espartoensis Pessagno.
11. 261-12-2, 11-12, $\times 50$.

12. 261-16-1, 101-103.

Figures 13-15 ?Tripodictya elegantissima Vinassa.
13. 261-12-2, 11-12.
14. $261-13, \mathrm{CC}$.
15. 261-16-1, 101-103. 
Plate 1

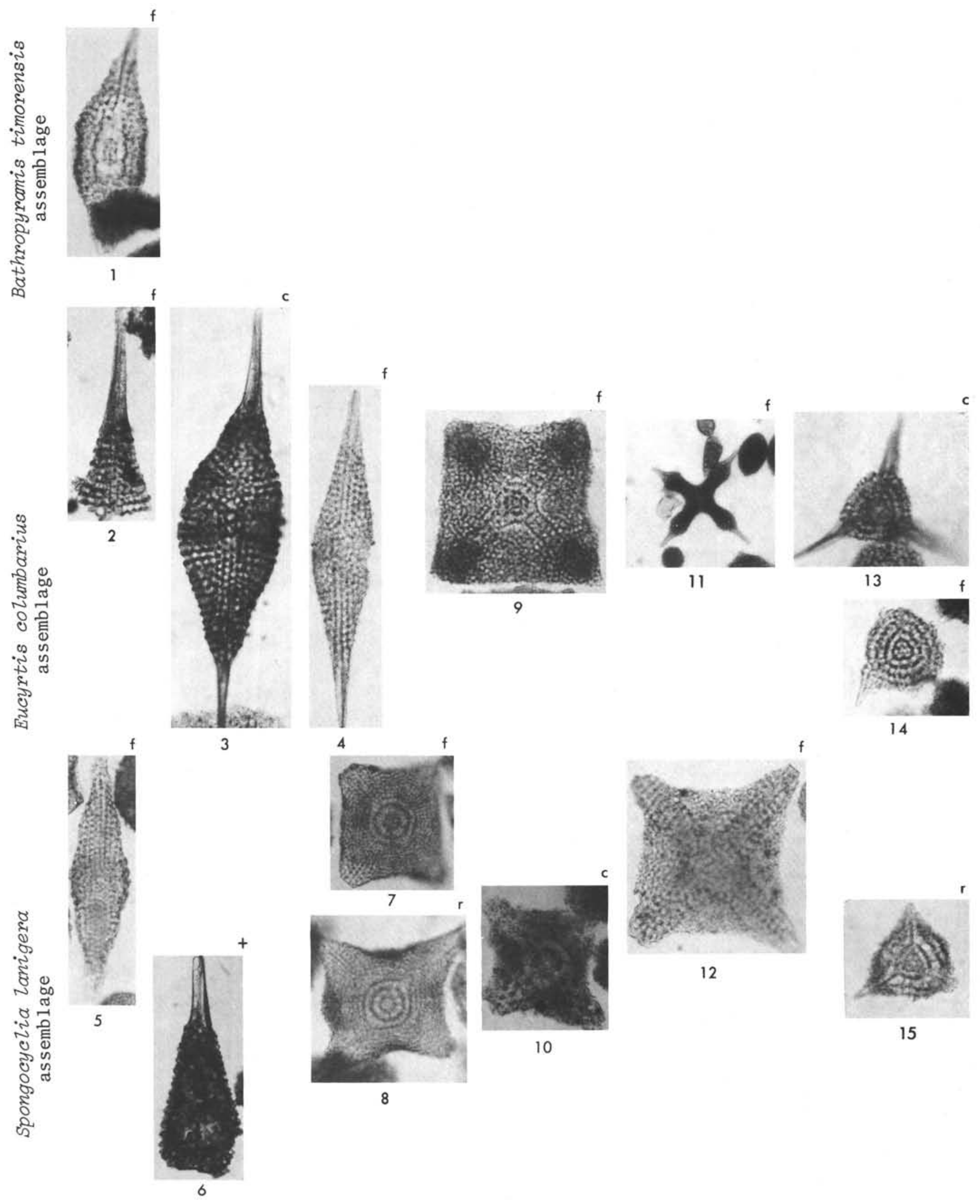




\section{PLATE 2}

(Magnification $\times 150$, unless otherwise indicated)

Figures 1-4 Spongosaturnalis lateralis (Campbell and Clark).

1. 261-9-4, 50-52.

2. $261-12, \mathrm{CC}, \times 135$.

3. 261-15-1, 9-11.

4. $261-23, \mathrm{CC}$.

Figure $5 \quad$ Spongosaturnalis polymorphus (Squinabol); 26112 , CC, $\times 50$.

Figures 6-8 ?Conosphaera tuberosa Tan Sin Hok.

6. 261-9-4, 50-52.

7. $261-12, \mathrm{CC}, \times 135$.

8. 261-15-1, 9-11, $\times 135$.

Figures 9-12 Xiphosphaera umbilicata Rüst.
9. 261-9, CC.
10. 261-12-2, top.
11. 261-13, CC.
12. $261-16-1,101-103$.

Figures 13-16 Sphaerostylus lanceola (Parona).

(?)13. 261-9-4, 110-112.

14. $261-10-1,10-12$.

15. 261-16-1, 101-103.

16. $261-23$, CC.

Figures 17, 18 Stylosphaera pusilla Campbell and Clark.

17. 261-12-2, top, $\times 135$.

18. 261-16-1, 101-103.

Figures 19-22 Spongoprunum sp. aff. Cyphantus probus Rüst.

19. 261-9, CC.

20. $261-10-1,10-12$.

21. 261-13, CC.

22. $261-23$, CC 


\section{PLATE 2}
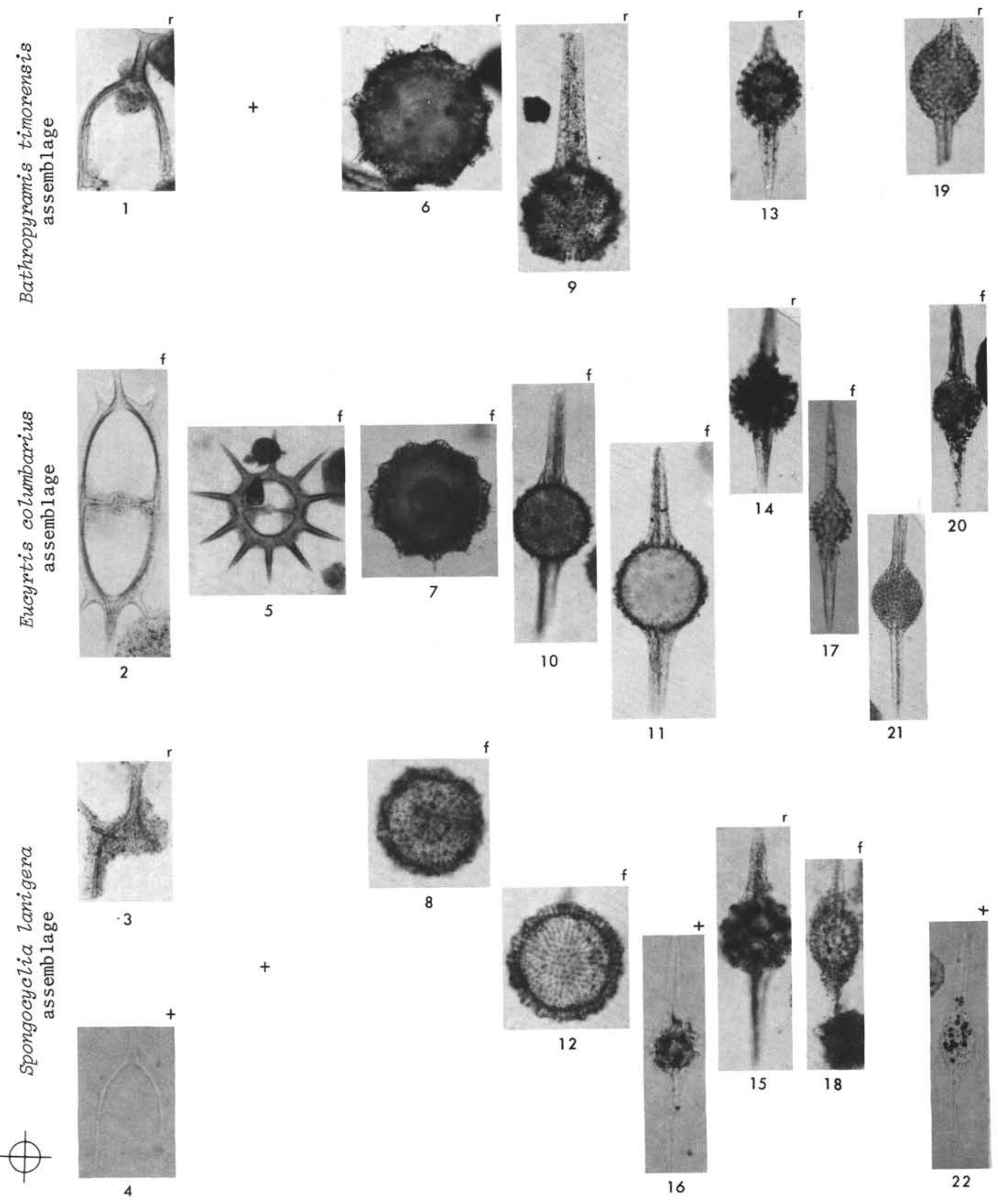
G. W. RENZ

\section{PLATE 3}

(Magnification $\times 150$ )

Figures 1a, lb Spongopyle stauromorphos n. sp. (a) 261-9, CC: (b) 261-12-2, top.

Figures 2-6 Spongopyle ecleptos $\mathrm{n}$. $\mathrm{sp}$.

2. 261-12, CC.

3. $261-13, \mathrm{CC}$.

4. 261-15-1, 9-11.

5. 261-16-1, 101-103.

6. 261-23, CC.

Figures 7,8 Spongopyle insolita Kozlova.

7. 261-15-1, 9-11.

8. 261-23, CC.

Figure 9 Spongodiscid 1 gen. and sp. indet.; 261-12, CC.

Figures 10,11 Rhopalodictyum sp.

10. 261-12, CC.

11. 261-15-1, 9-11.

Figure $12 \quad$ Spongodiscus sp. cf. S. americanus Kozlova; 26112-2, 11-12. 
PLATE 3
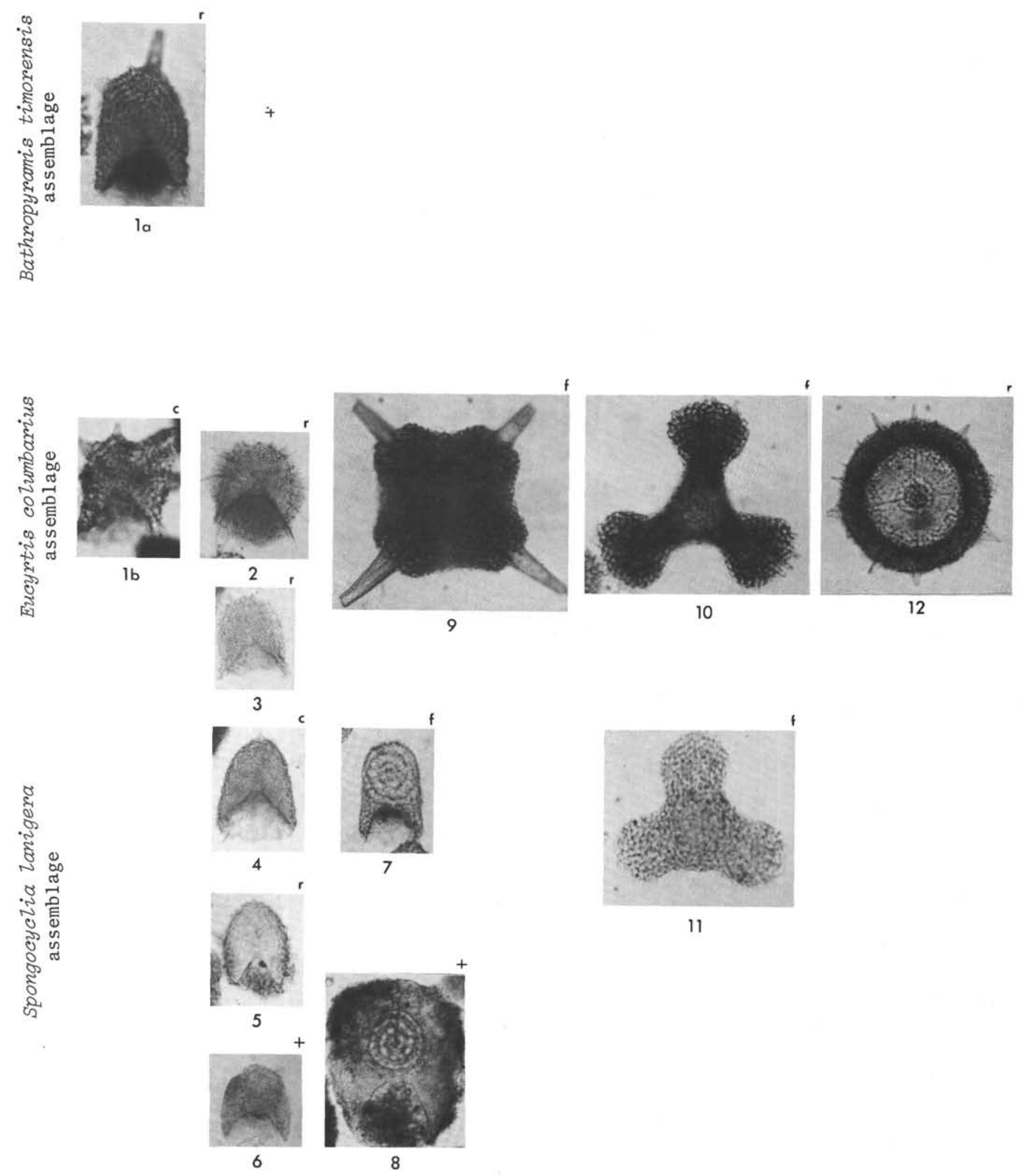
PLATE 4

(Magnification $\times 150$, unless otherwise indicated)

Figures 1-4 Spongocyclia trachodes n. sp.

1. 261-12, CC.

2. 261-13, CC.

3. 261-15-1, 9-11.

4. 261-23, CC.

Figure 5 Spongocyclia lanigera n. sp.; 261-15, CC.

Figures 6, 7 Lithocyclia? sp. A.

6. 261-15, CC.

7. 261-16-1, 101-103.

Figures 8,9 Bathropyramis timorensis n. sp.

8. 261-9-4, 110-112.

9. 261-9, CC.

Figures 10-14 Cornutella spp.

10. Cornutella californica var. A Campbell and Clark; 261-12-2, 11-12.

11. Cornutella californica var. B Campbell and Clark; 261-12, CC, $\times 135$.

12. Cornutella sp.; 261-16-1, 101-103.

13. Cornutella sp.; 261-16-1, 101-103.

14. Cornutella sp.; 261-23, CC, $\times 260$.

Figures 15, 16 ?Cyrtocalpis operosa Tan Sin Hok.

15. 261-13, CC.

16. 261-16-1, 101-103. 


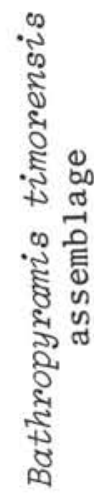

PLATE 4
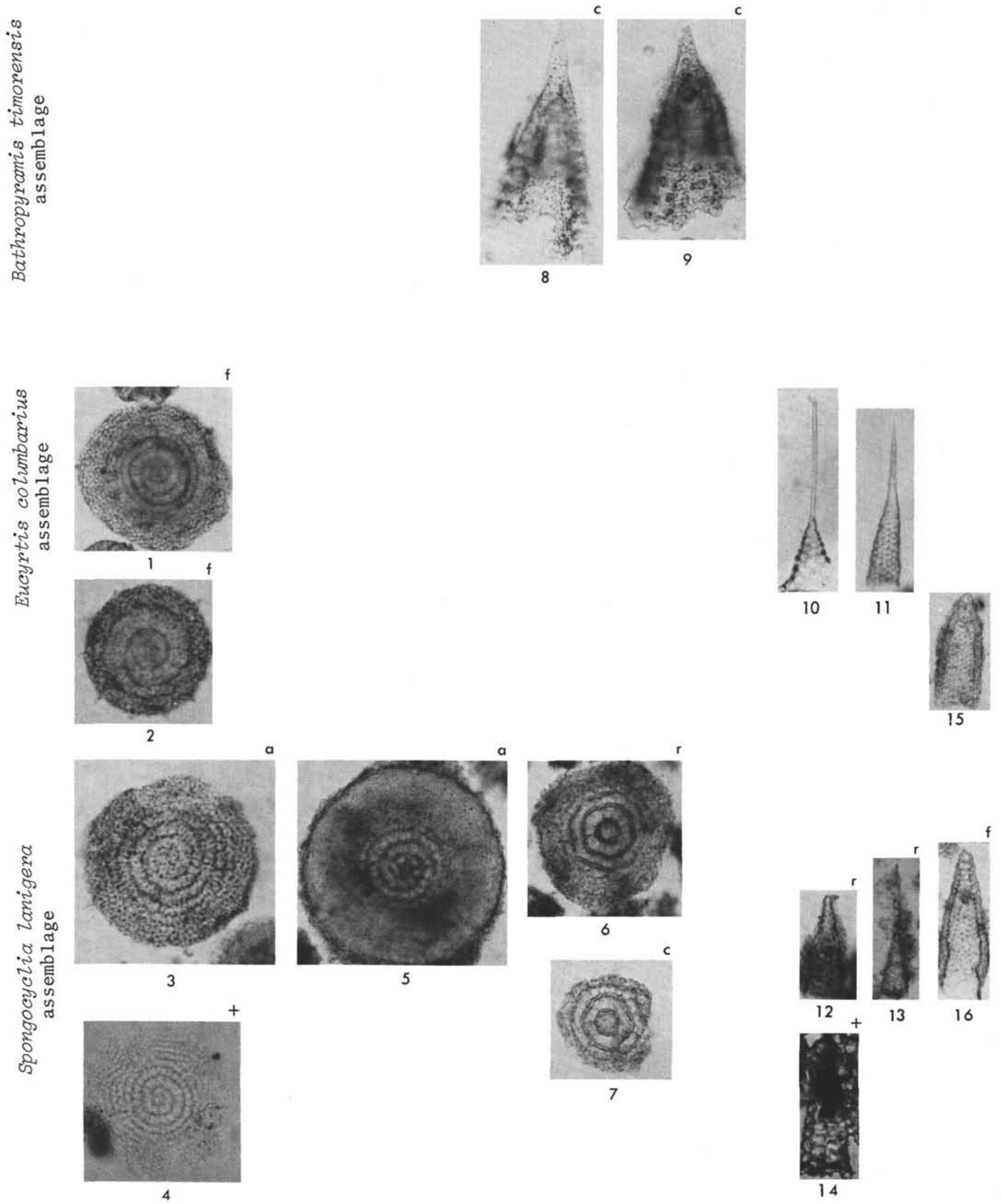
PLATE 5

(Magnification $\times 150$, unless otherwise indicated)

Figures 1-3 Amphipyndax (?) epiplatys n. sp.

1. 261-9, CC.

2. $261-12, \mathrm{CC}$.

3. 261-16-1, 101-103.

Figures 4-6 Amphipyndax (?) pyrgodes $\mathrm{n}$. $\mathrm{sp}$.
4. 261-9, CC.
5. $261-12, \mathrm{CC}$.
6. 261-13, CC.

Figures 7-9 Amphipyndax mediocris (Tan Sin Hok)

7. 261-12, CC.

8. 261-15-1, 9-11, $\times 170$.

9. 261-23, CC.

Figures 10-13 Hemicryptocapsa pseudopilula Tan Sin Hok

10. 261-9, CC.

11. 261-12-2, top:

12. $261-13, \mathrm{CC}$.

13. 261-23, CC,

Figures 14-16 Dictyophimus gracilis Tan Sin Hok

14. 261-12-2, top, $\times 135$.

15. 261-16-1, 101-103.

16. $261-23, \mathrm{CC}$.

Figure 17 Dictyophimus obliquum (Hinde); 261-12-2, 11-12, $\times 135$.

Figures 18, 19 ?Tripocalpis ellyae Tan Sin Hok

18. $261-12$, CC.

19. $261-23, \mathrm{CC}$. 


\section{PLATE 5}
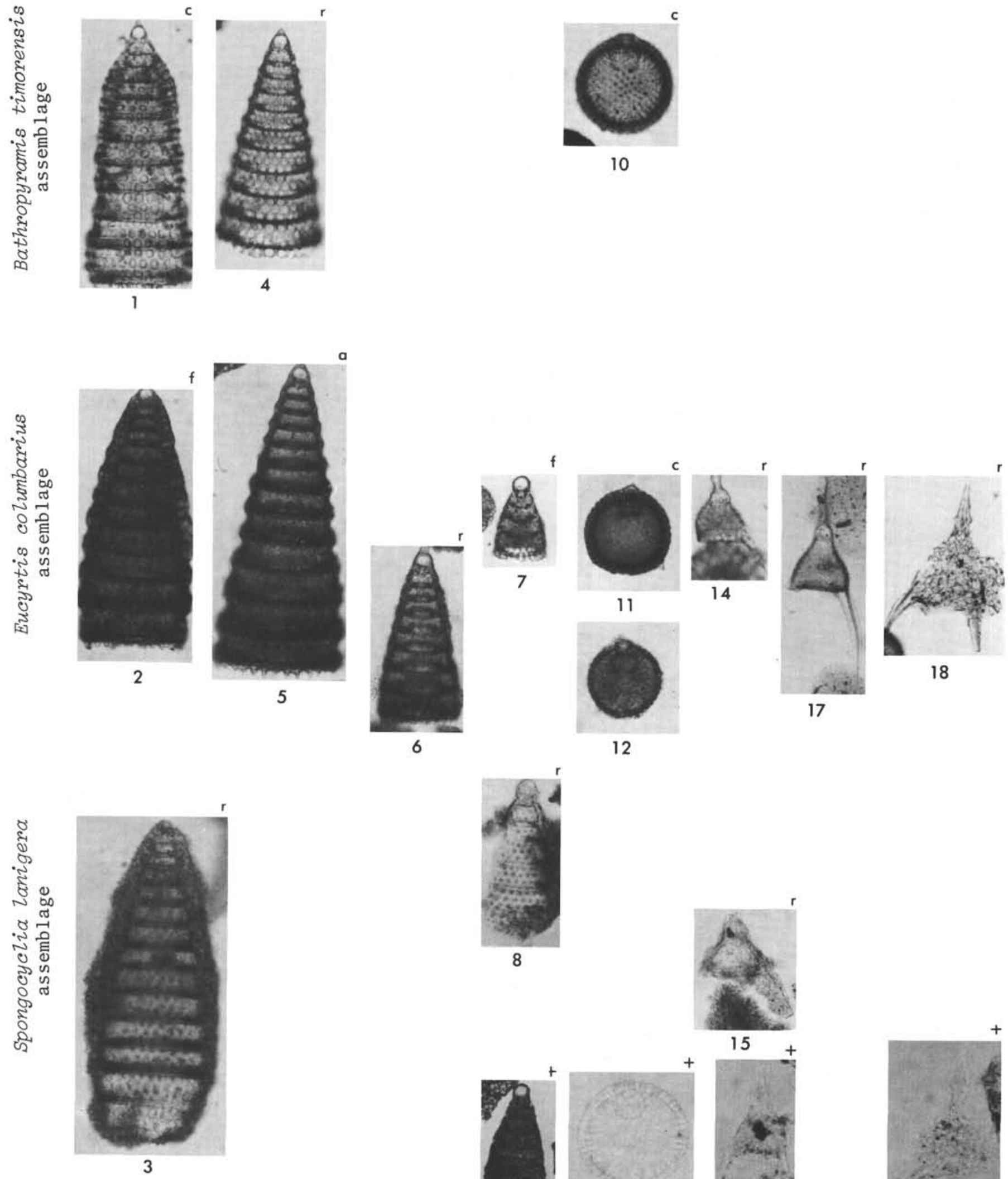

12
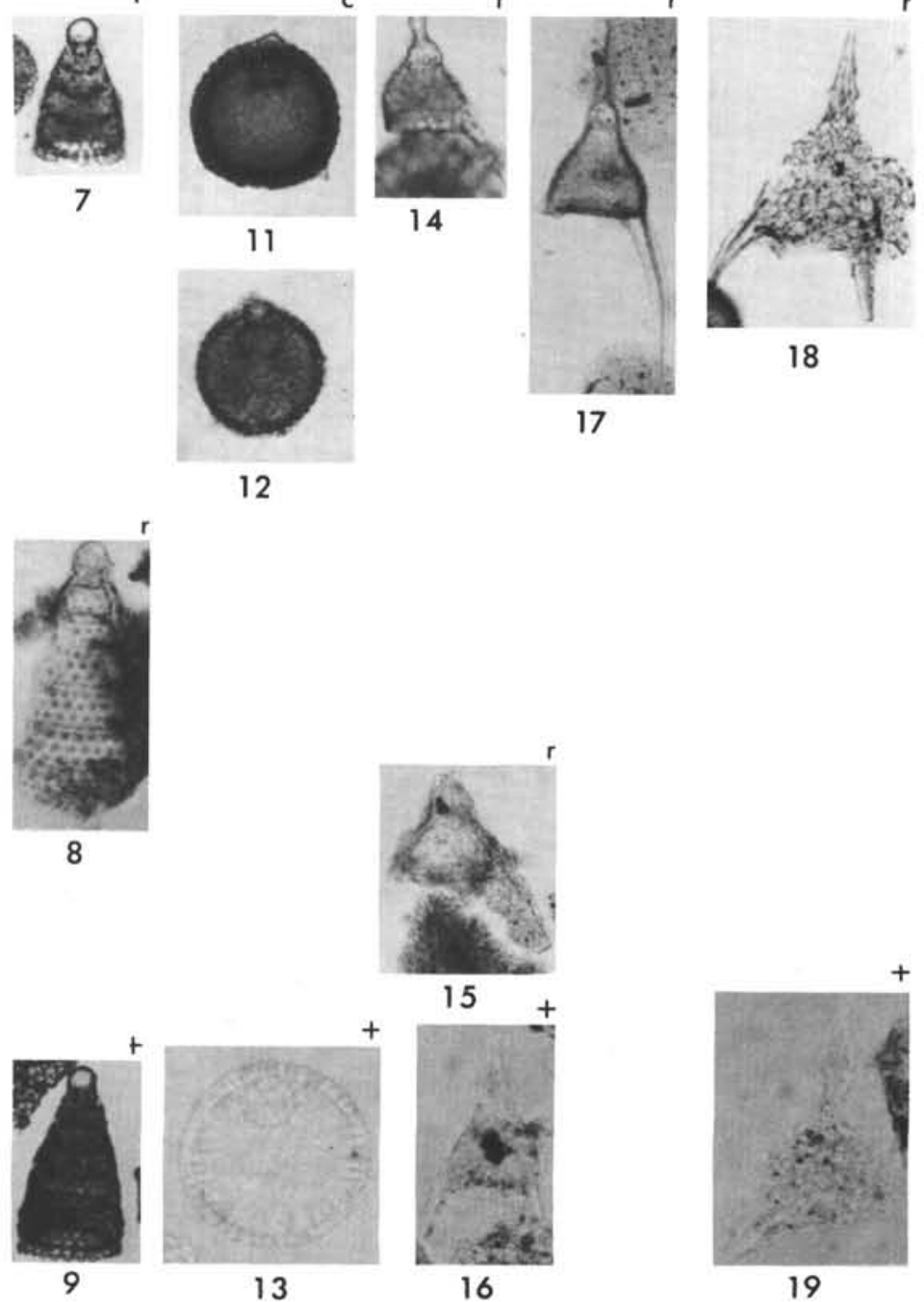


\section{PLATE 6 \\ (Magnification $\times 150$, unless otherwise indicated)}

Figures 1-3 ?Cyrtocapsa grutterinki var. $\alpha$ Tan Sin Hok.

1. 261-9-4, 50-52.

2. 261-12, CC, $\times 135$.

3. $261-23, \mathrm{CC}$.

Figures 4-7 Theocorys sp. aff. T. antiqua Squinabol.

4. 261-9-4, 110-112.

5. 261-12, CC.

6. 261-13, CC:

7. 261-16-1, 101-103.

Figures 8-12 ?Tricolocapsa parvipora Tan Sin Hok.
8. 261-9-3, 90-92.
9. $261-9$, CC.
10. $261-10-1,10-12$.
11. 261-12-2, top, $\times 135$.
12. 261-16-1, 101-103.

Figures 13-18 Dicolocapsa verbeeki Tan Sin Hok.
13. 261-9-3, 90-92.
14. $261-9-4,110-112$.
15. 261-10-1, 10-12.
16. $261-12-2,11-12, \times 135$.
17. $261-13, \mathrm{CC}$.
18. 261-23, CC.

Figure 19 Dicolocapsa sp.; 261-9, CC.

Figure 20 ?Theocapsa elata Tan Sin Hok; 261-9, CC.

Figures 21-23 Artocapsa bicornis Tan Sin Hok.

21. 261-9, CC

22. $261-15-1,9-11$.

23. $261-23, \mathrm{CC}$.

Figure 24 ?Artocapsa ultima Tan Sin Hok; 261-9-4, 110-112. 
PLATE 6
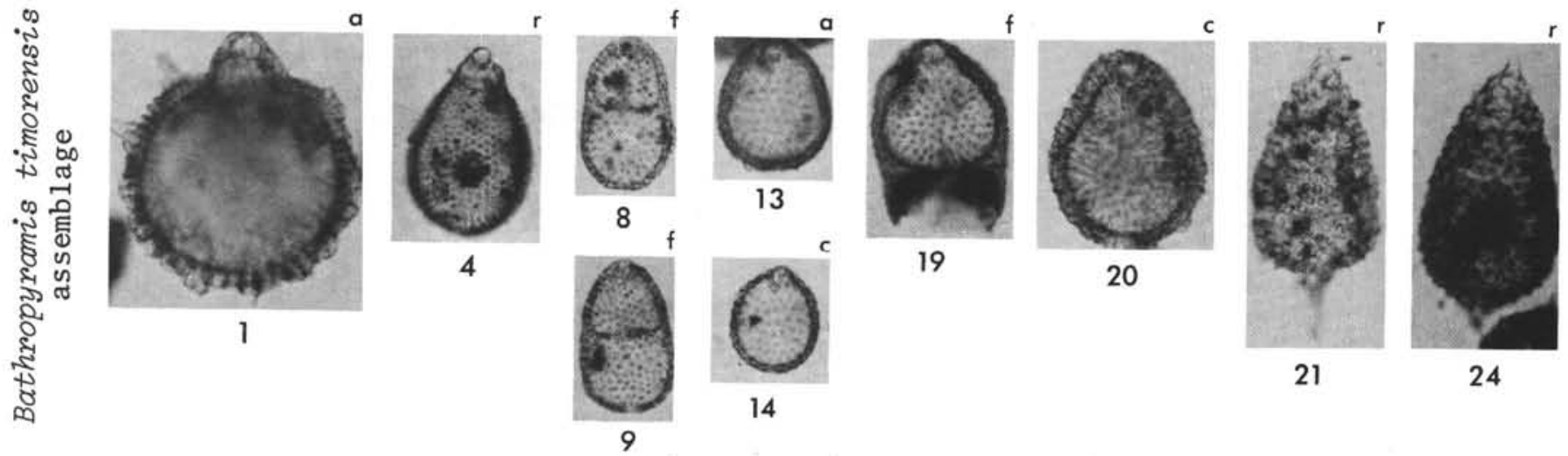

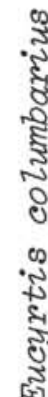
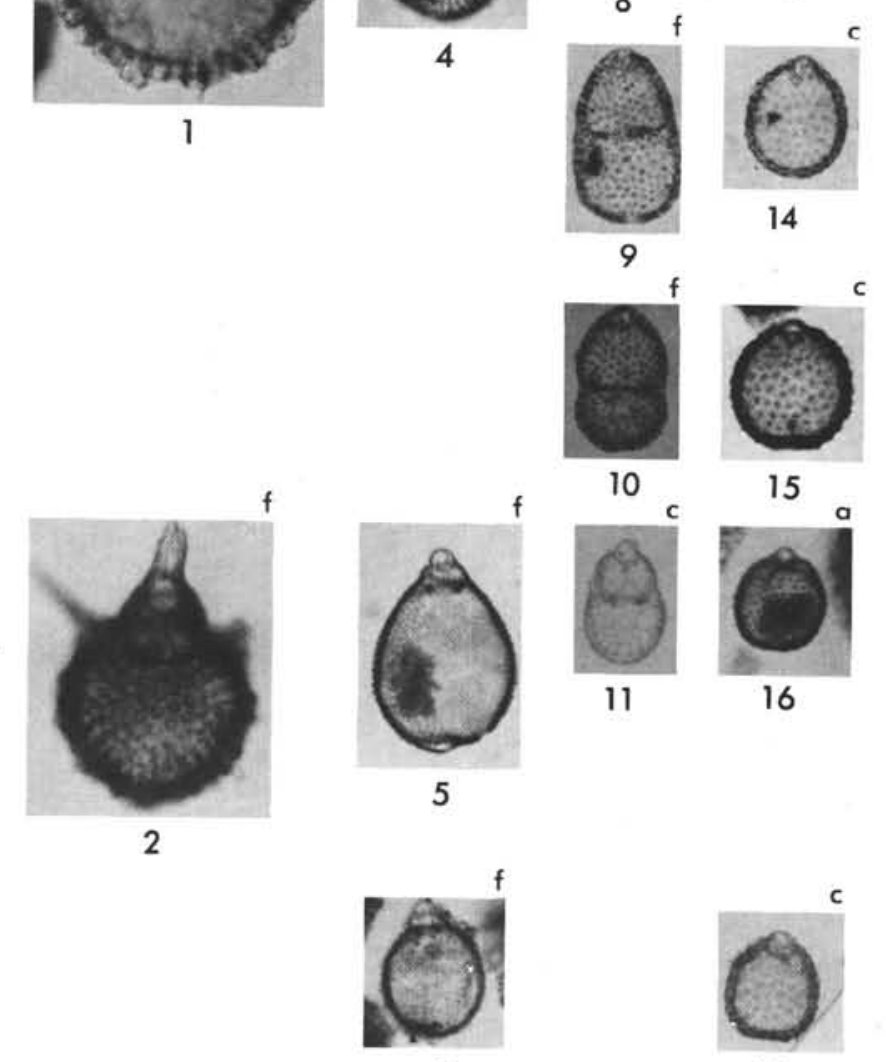

6

17

ह
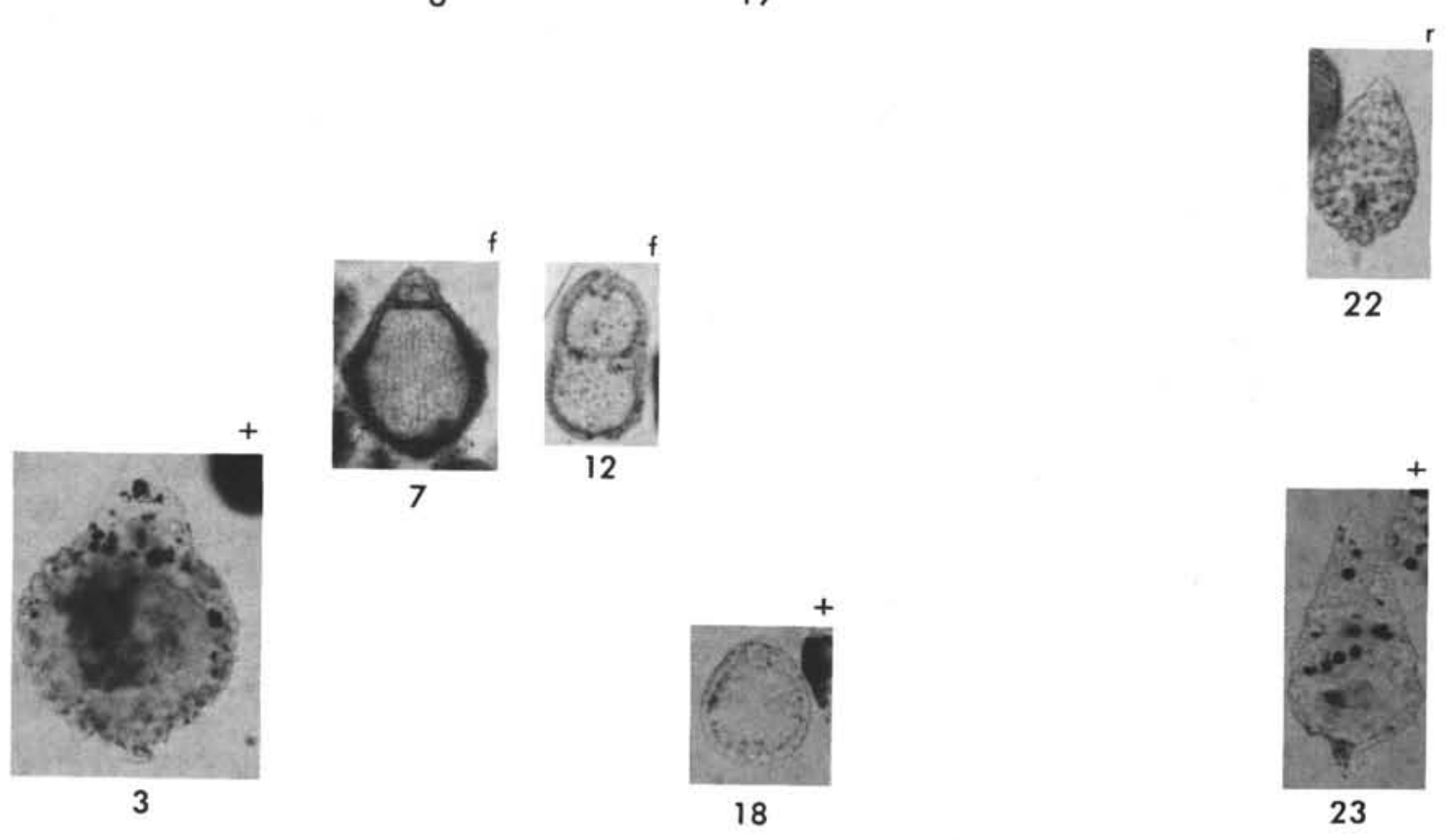

23 
PLATE 7

(Magnification $\times 150$, unless otherwise indicated)

Figures 1-4 ?Eucyrtis molengraaffi (Tan Sin Hok).

1. 261-9-4, 50-52.

2. $261-12, C C, \times 135$.

3. $261-13, \mathrm{CC}$.

4. 261-16-1, 101-103.

Figures 5-7 Lithomitra pseudopinquis Tan Sin Hok.
5. 261-12, CC, $\times 135$.
6. $261-13, \mathrm{CC}$.
7. $261-23, \mathrm{CC}$.

Figures 8-11 ?Stichocapsa procera Hinde.
8. 261-9, CC.
9. $261-12, \mathrm{CC}$.
10. 261-15-1, 9-11.
11. $261-23, \mathrm{CC}$.

Figures 12, 13 Lithostrobus erectus Tan Sin Hok.
12. 261-10-1, 10-12.
13. $261-12, \mathrm{CC}$.

Figures 14-20 Eucyrtis columbarius n. sp.
14. 261-10, CC.
16. 261-12, CC.
17. $261-13, \mathrm{CC}$.
18. 261-15-1, 9-11.
19. 261-23, CC.
20. 261-23, CC.

15. 261-12-2, top, $\times 135$.

Figures 21-25 Eucyrtis hanni (Tan Sin Hok).

21. 261-12-2, top, $\times 135$.

22. 261-12-2, 11-12.

23. 261-13, CC.

24. 261-15-1, 9-11.

25. 261-23, CC.

Figures 26-29 Eucyrtis bulbosus n. sp.

26. 261-12-2, 11-12.

27. 261-13, CC.

28. 261-15-1, 9-11.

29. 261-16-1, 101-103.

Figure $30 \quad$ Lithocampe chenodes $n$. sp.; 261-12-2, top, $\times 135$. 
PLATE 7
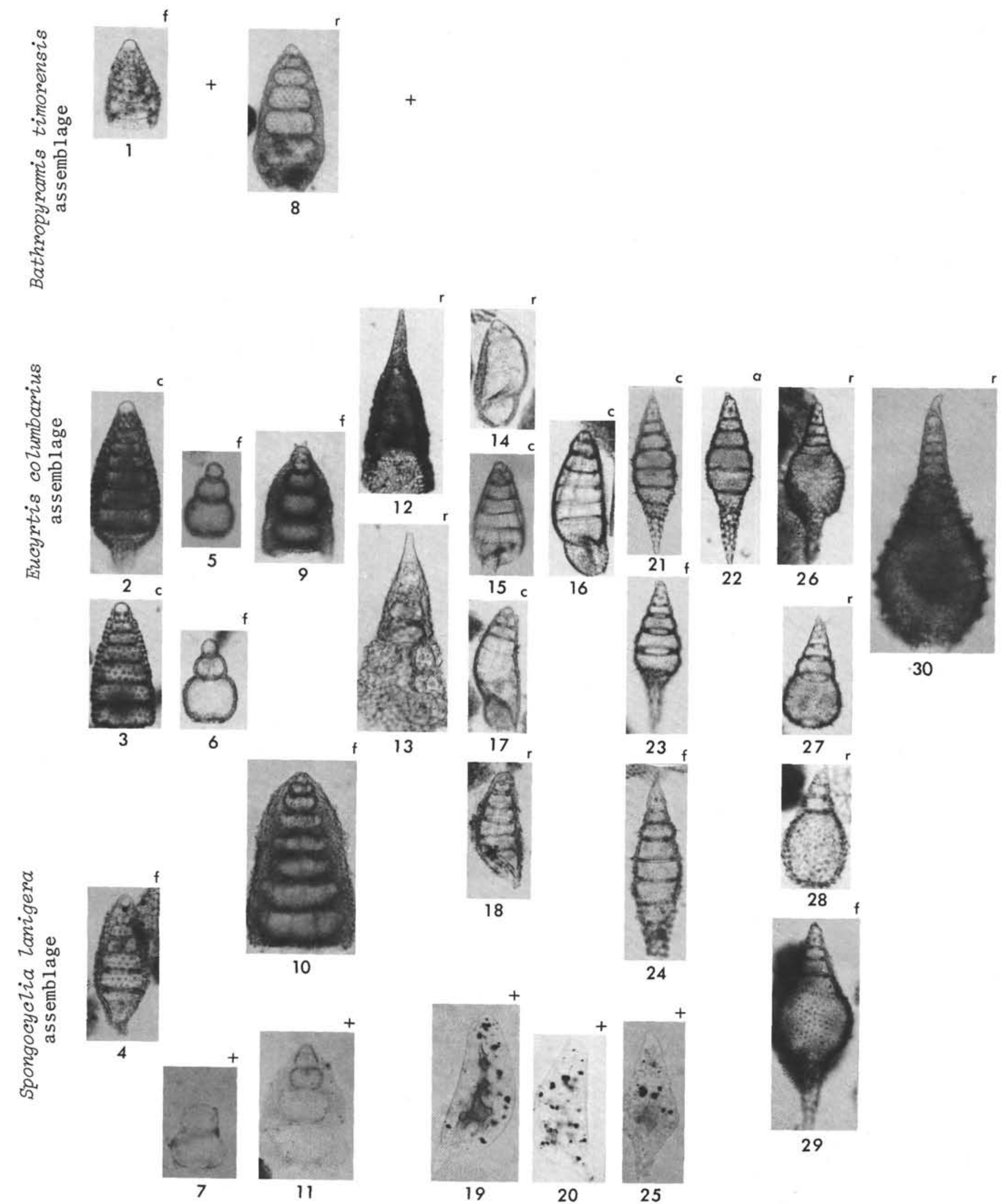


\section{PLATE 8 \\ (Magnification $\times 150$, unless otherwise indicated)}

Figures 1-4 Dictyomitra lilyae Tan Sin Hok.

1. 261-9-4, 50-52.

2. $261-10-1,10-12$.

3. $261-13, \mathrm{CC}, \times 135$.

4. 261-15-1, 9-11.

Figures 5,6 Dictyomitra pseudoscalaris (Tan Sin Hok).

5. 261-9-4, 110-112.

6. 261-13, CC, $\times 135$.

Figures 7,8 Dictyomitra excellens (Tan Sin Hok).

7. 261-12-2, top, $\times 135$.

8. 261-16-1, 101-103, $\times 135$.

Figures 9-13 Dictyomitra brouweri var. $\gamma$ (Tan Sin Hok).
9. 261-9, CC
10. $261-12, \mathrm{CC}$.
11. 261-15-1, 9-11.
12. 261-16-1, 101-103.
13. $261-23, \mathrm{CC}$.

Figures 14-16 Dictyomitra brouweri var. $\alpha$ (Tan Sin Hok).
14. 261-9, CC.
15. $261-12-2,11-12$.
16. $261-23$, CC.

Figures 17-19 Eucyrtidium vermiculatum $\mathrm{n}$. sp.

17. 261-12-2, top.

18. 261-13, CC.

19. 261-15-1, 9-11.

Figure 20 ?Dictyomitra malleola Aliev; 261-15-1, 9-11.

Figures 21, 22 Eucyrtidium (?) boodes $\mathrm{n}$. sp.

21. 261-9-4, 110-112.

22. $261-9-4,110-112$. 


\section{PLATE 8}

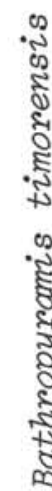
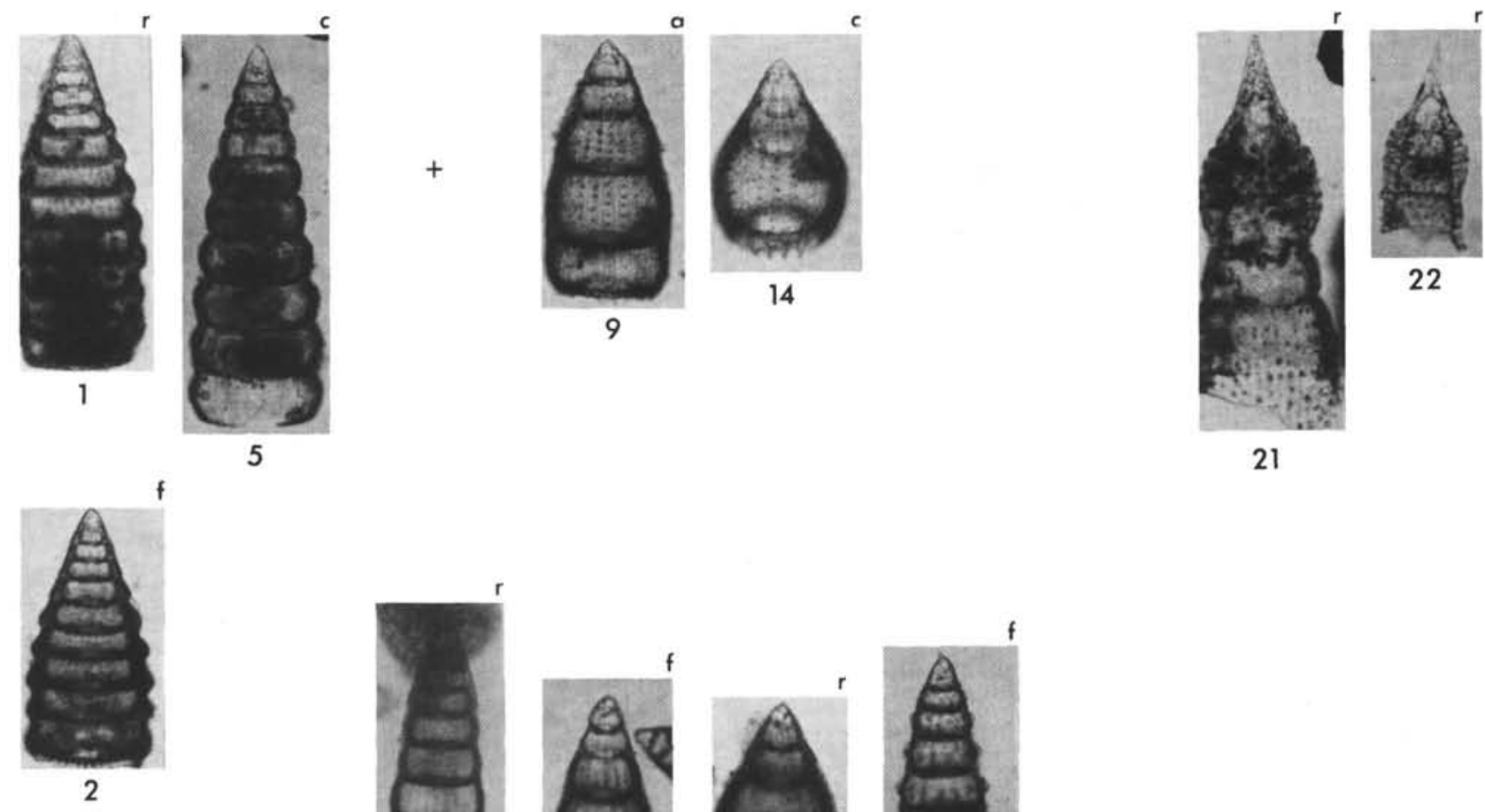

त त

ฐิ
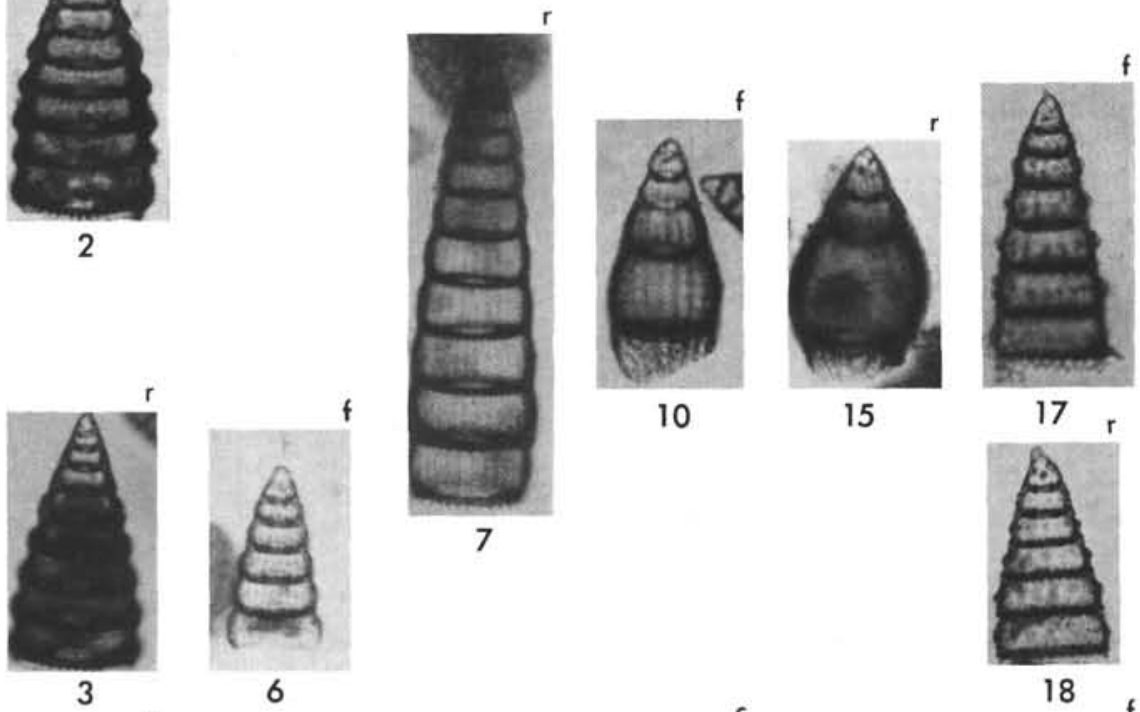

15
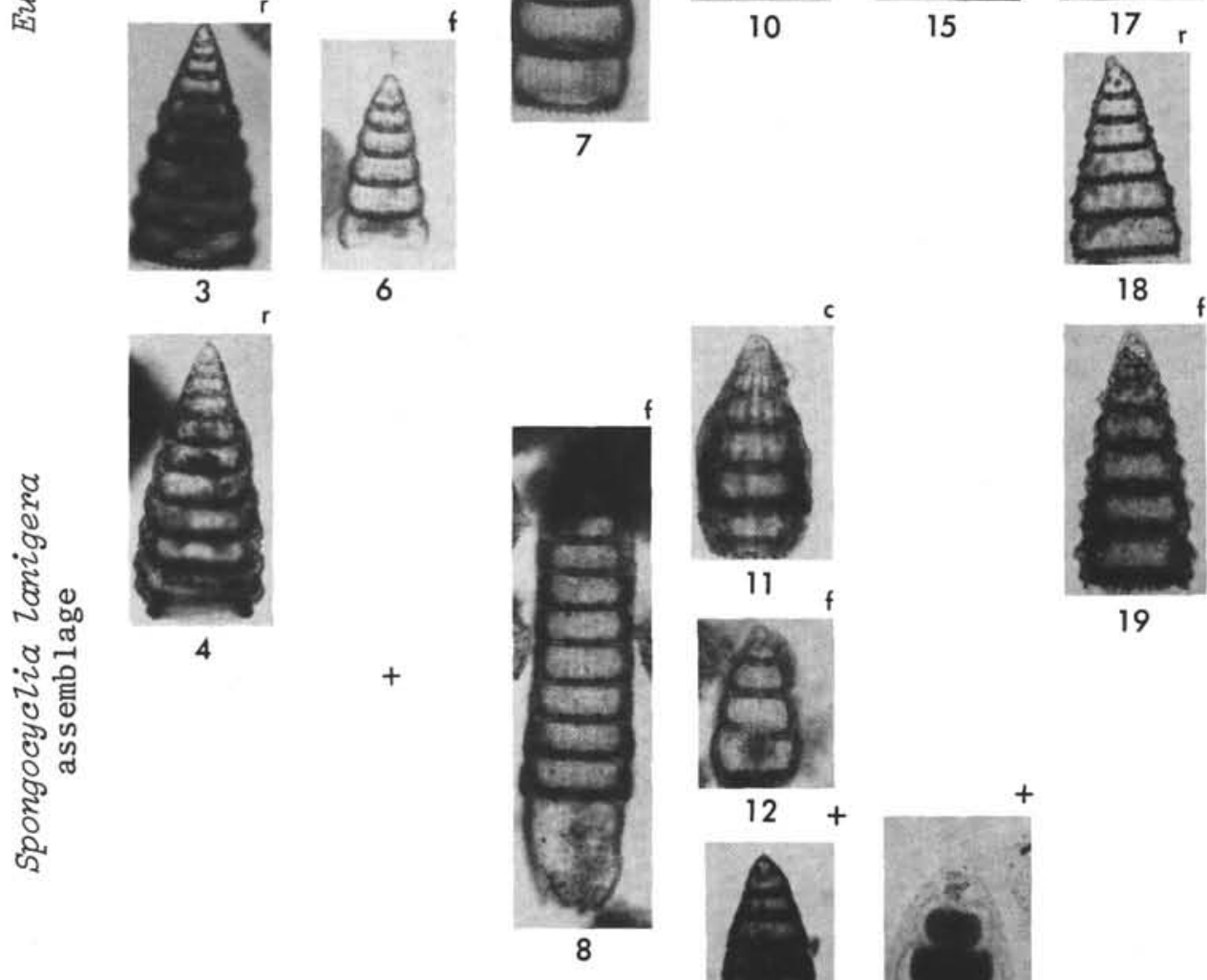

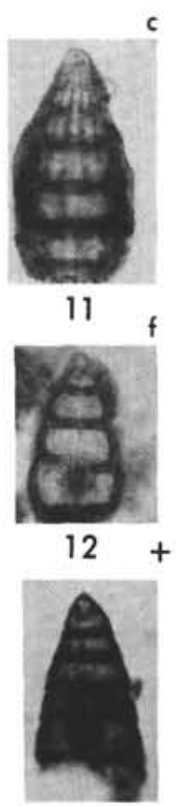

13

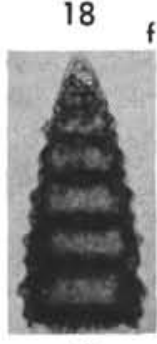

19

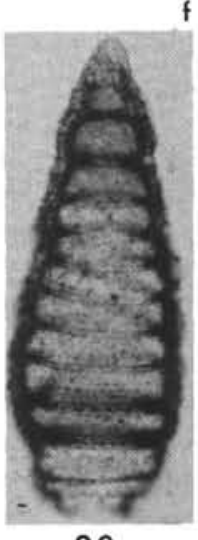

20 
b. PLATE 9

(Magnification $\times 150$, unless otherwise indicated)

Figure 1 Amphibrachium (?) hastatum n. sp.; holotype, 26112, CC.

Figure 2 Patulibracchium petroleumensis Pessagno; 261-12, CC.

Figure 3 Hagiastrid 1 gen. and sp. indet.; 261-15-1, 9-11.

Figure 4 Crucella espartoensis Pessagno; 261-12-2, top.

Figure 5 ?Tripodictya elegantissima Vinassa; 261-12-2, top.

Figure 6 Hagiastrid 2 gen. and sp. indet., 261-12-2, top.

Figure 7 Porodiscus sp. aff. P. delicatulus (Lipman); 261-122, top.

Figure 8 Haliomma minor Campbell and Clark; 261-12-2, 11-12.

Figure 9 Tholodiscus fresnoensis (Foreman); 261-12-2, top.

Figure 10 incerti sedis; 261-12-2, top.

Figure 11 Amphibrachium sp. cf. A. concentricum Lipman; 261-15-1, 9-11.

Figure 12 ?Halesium quadratum Pessagno; 261-12-2, 11-12.

Figure 13 Xiphodictya sp. cf. X. ovalis Rüst; 261-9-3, 90-92.

Figure 14 Porodiscus delicatulus (Lipman); 261-9, CC.

Figure 15 Spongosaturnalis sp. aff. Saturnalis lateralis Campbell and Clark; 261-12, CC, $\times 170$.

Figure 16 Actinommid 4 gen. and sp. indet.; 261-12-2, top.

Figure 17 ?Conosphaera tuberosa Tan Sin Hok; 261-12-2, top.

Figure 18 Staurostylus italicus Rüst; 261-12, CC.

Figure 19 Sphaerostylus lanceola (Parona); 261-15-1, 9-11.

Figure 20 Stylosphaera pusilla Campbell and Clark; 261-122, top.

Figure $21 \quad$ Xiphosphaera umbilicata Rüst; 261-12-2, top.

Figure 22 Spongosaturnalis sp. aff. Saturnalis polymorphus Squinabol; 261-12, CC, $\times 110$. 
PLATE 9

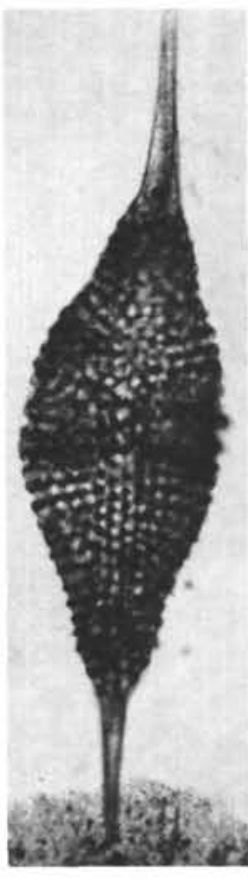

1

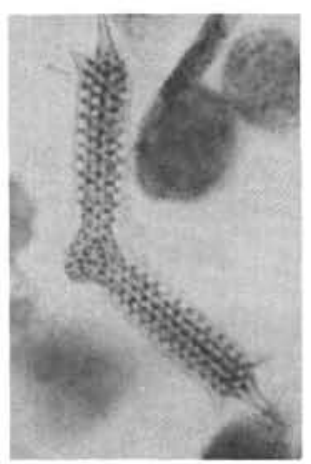

12

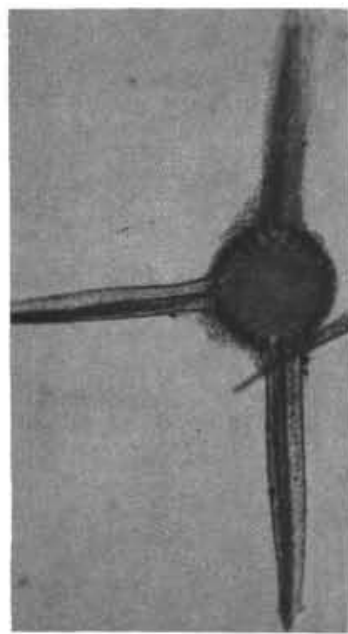

18
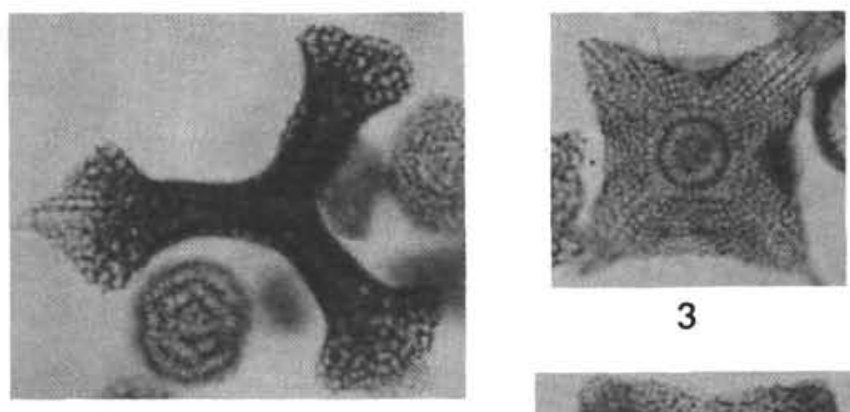

3

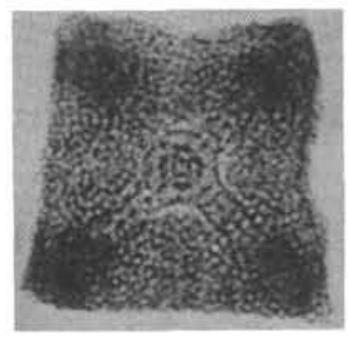

6
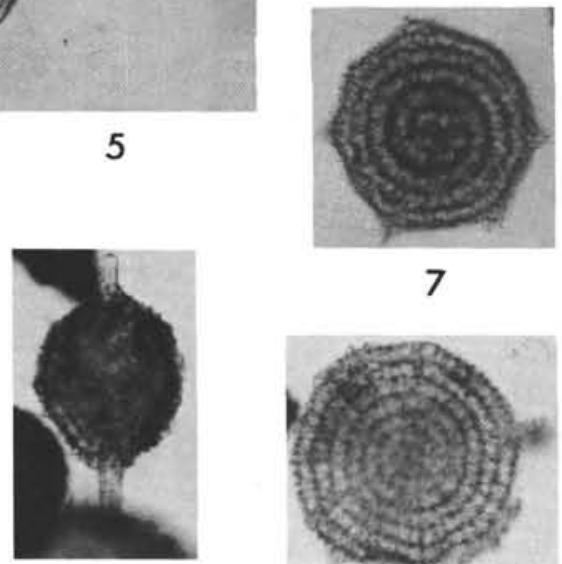

13

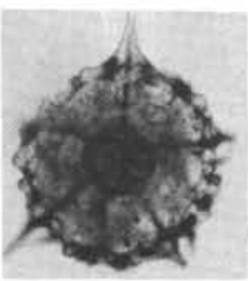

16

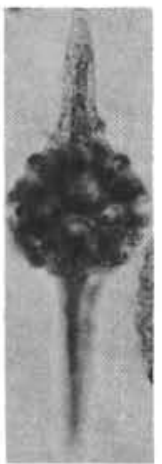

19

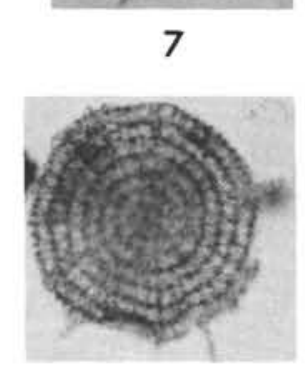

14

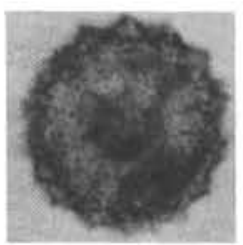

17

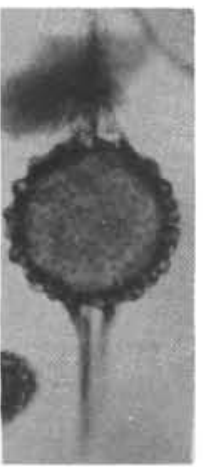

21

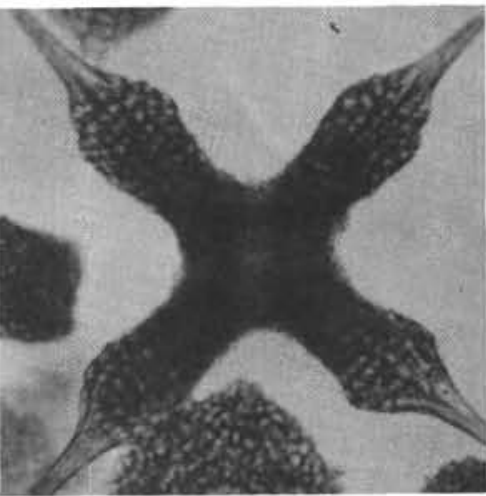

4

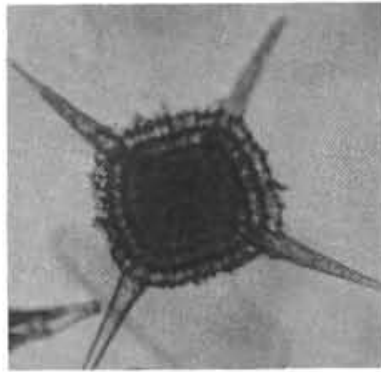

9

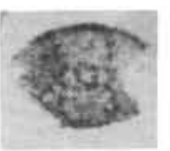

10

11

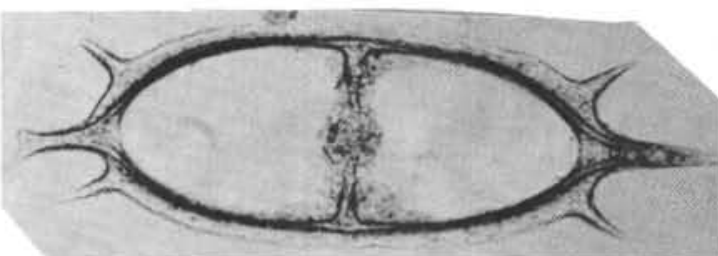

15

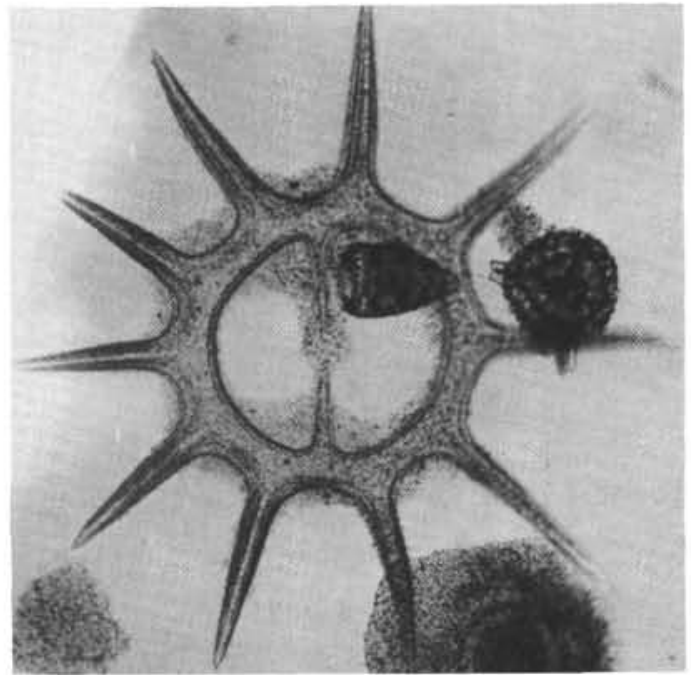

22 


$$
\begin{gathered}
\text { PLATE } 10 \\
\text { (Magnification } \times 150 \text { ) }
\end{gathered}
$$

Figure 1

Figure 2

Figure 3

Figure 4

Figure 5

Figure 6

Figure 7a

Figure $7 b$

Figure 8

Figure 9

Figure 10

Figure 11

Figure 12

Figure 13

Figure 14

Figure 15

Figure 16

Figure 17

Figure 18

Figure 19

Figure 20

Figure 21

Figure 22

Figure 23
Stylotrochus sp. 261-12-2, 11-12.

Rhopalodictyum sp.; 261-12-2, top.

Spongotripus sp. cf. Tripodictya triacuminata Lipman; 261-12-2, top.

Spongodiscid 1 gen. and sp. indet.; 261-12-2, top.

?Spongotrochus polygonatus Campbell and Clark; 261-12-2, top.

Spongodiscus sp. cf. S. americanus Kozlova; 26112-2, top.

Spongocyclia lanigera $\mathrm{n}$. sp.; Side view, 261-15, CC.

Spongocyclia lanigera $\mathrm{n}$. sp.; holotype, 261-15, CC.

Spongopyle galeata $\mathrm{n}$. sp.; holotype, 261-12-2, top.

Spongopyle stauromorphos n. sp.; holotype, 261-9, CC.

Spongopyle insolita Kozlova; 261-9, CC.

Spongolonche sp. aff. $S$. grandis Campbell and Clark; 261-9, CC.

?Spongolonche grandis Campbell and Clark; 261-9-

4, 110-112.

Spongocyclia trachodes n. sp.; holotype, 261-15-1, 9-11.

Spongopyle ecleptos $\mathrm{n}$. sp.; holotype, 261-12-2, top.

Spongopyle trabeata $\mathrm{n}$. sp.; holotype, 261-15-1, 911.

Lithocyclia? sp. A; 261-15, CC.

Spongodiscus sp. 1; 261-12-2, top.

Spongoprunum diversispina Squinabol; 261-12-2, top.

Spongoprunum sp. aff. Cyphantus probus Rüst; 261-12-2, 11-12.

Spongodiscid 2 gen. and sp. indet.; 261-12-2, top.

?Stylotrochus antiquus Campbell and Clark; 26112-2, top.

Spongodiscus sp. 2; 261-15-1, 9-11.

Spongodiscid 3 gen. and sp. indet.; 261-15, CC. 
PLATE 10

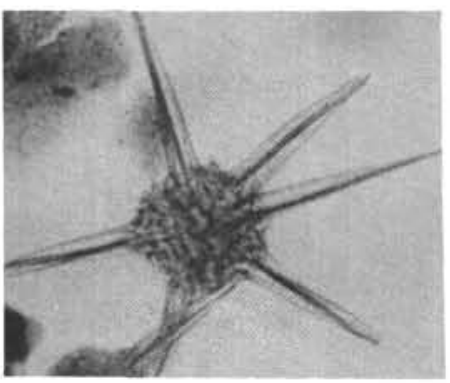

1

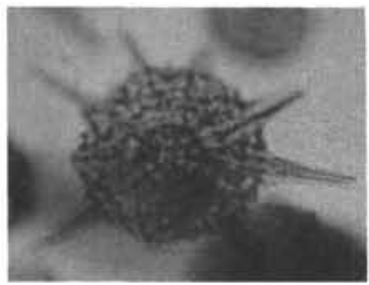

5

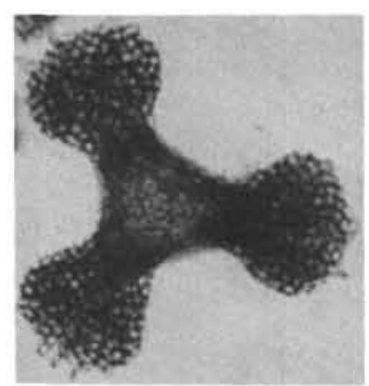

2

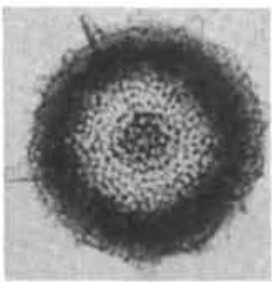

6

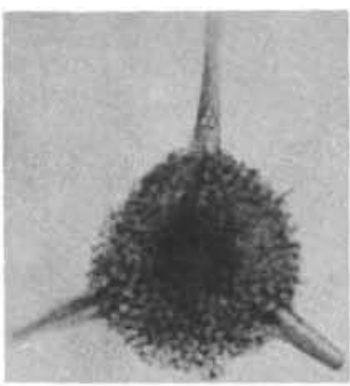

3

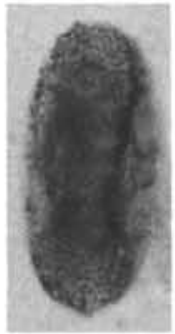

$7 a$

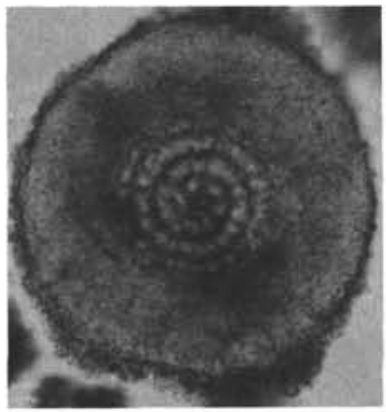

7 b
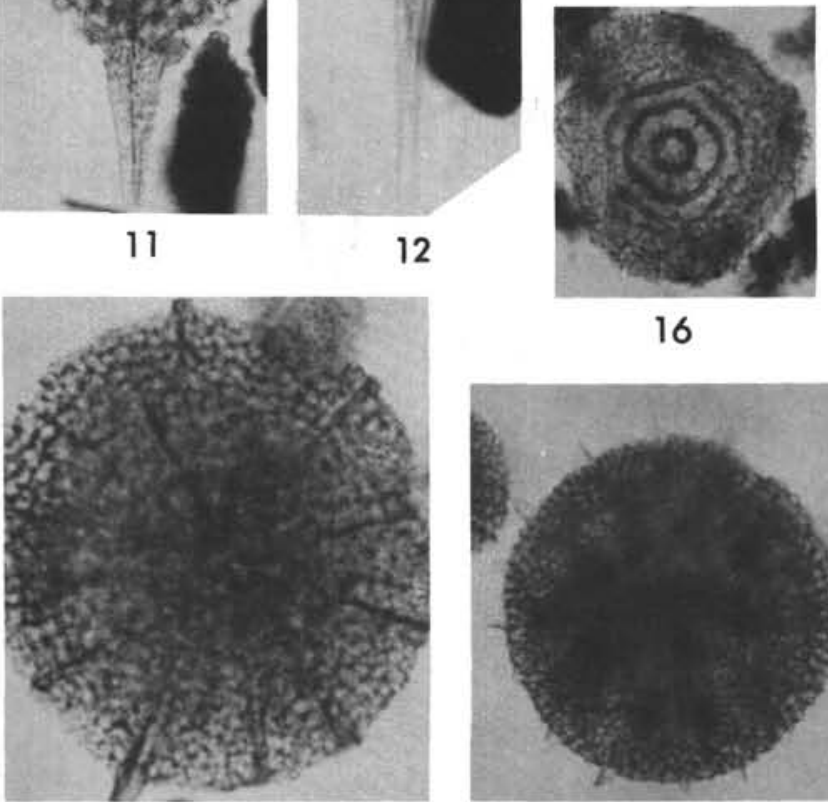

20
16

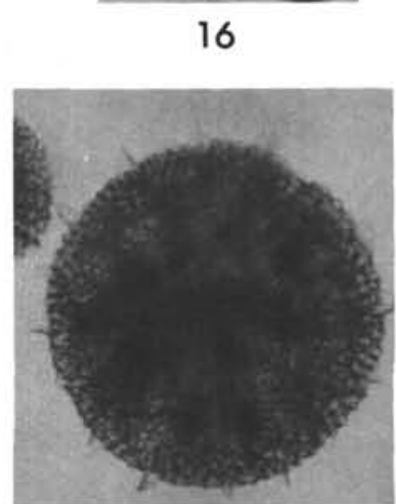

21

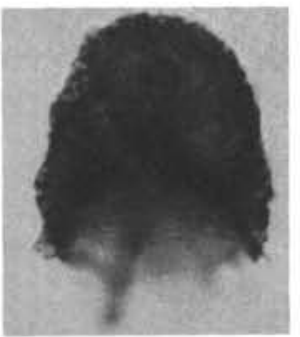

8

$$
14
$$

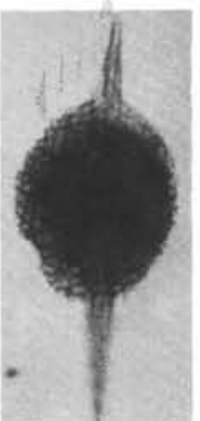

18

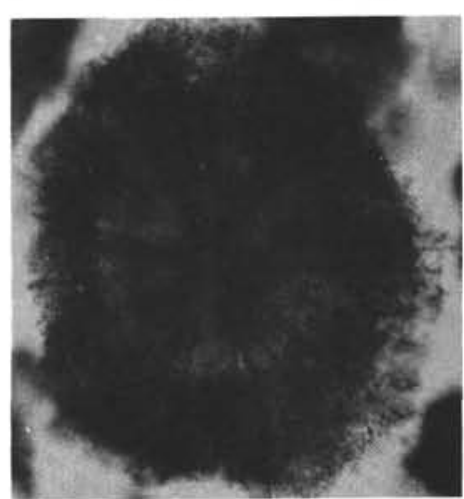

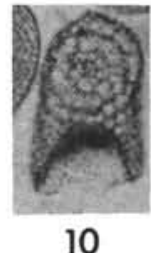

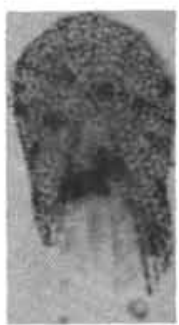

15.

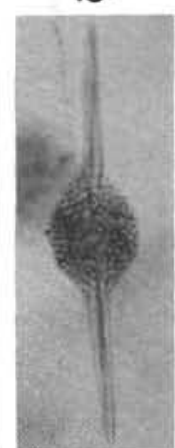

19

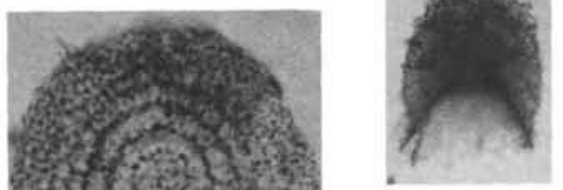

22

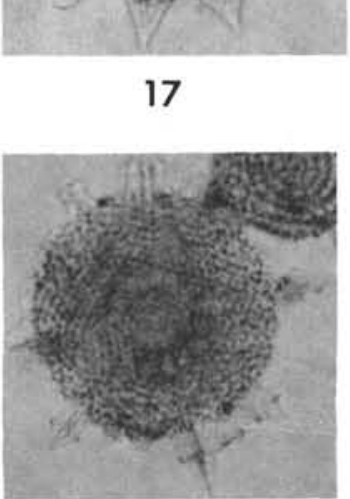


Figure 1

Figure 2

Figure 3

Figure 4

Figure 5

Figure 6

Figure 7

Figure 8

Figure 9

Figure 10

Figure 11

Figure 12a

Figure 12b

Figure 13

Figure 14

Figure 15a

Figure 15b

Figure 16

Figure 17

Figure 18

Figure 19a

Figure 19b

Figure 20

Figure 21

Figure 22

Figure 23

Figure 24

Figure 25

Figure 26

Figure 27

Figure 28

Figure 29

Figure 30

Figure 31

Figure 32

Figure 33

Figure 34

Figure 35

\section{PLATE 11}

Dictyophimus obliquum (Hinde); 261-12-2, 11-12, ×70.

Dicolocapsa sp; 261-9, CC, $\times 135$.

?Tricolocapsa parvipora Tan Sin Hok; 261-12-2, top, $\times 135$.

Theocorys sp. aff. T. antiqua Squinabol; 261-9-3, 90-92, $\times 150$.

?Stichocapsa rutteni Tan Sin Hok; 261-9, CC, ×135.

?Stichocapsa sp. aff. S. rutteni Tan Sin Hok; 261-12-2, 11-12, $\times 135$.

?Cyrtocapsa grutterinki var. $\alpha$ Tan Sin Hok; 261-12-2, top, $\times 135$.

?Theocapsa elata Tin Sin Hok; 261-9-3, 90-92, ×135.

Dicolocapsa verbeeki Tan Sin Hok; 261-13, CC, ×135.

?Tripocalpis ellyae Tan Sin Hok; 261-12, CC, ×150.

Dictyophimus gracilis Tan Sin Hok; 261-12-2, top, $\times 135$.

Artocapsa bicornis Tan Sin Hok; 261-9-3, 90-92, ×150.

Artocapsa bicornis Tan Sin Hok; 261-9, CC, ×150.

?Artocapsa ultima Tan Sin Hok; 261-9, CC, $\times 135$.

?Squinabolella putahensis Pessagno; 261-12, CC, $\times 150$.

Eucyrtidium (?) boodes n. sp.; holotype, 261-9-4, 110-112, $\times 135$.

Eucyrtidium (?) boodes n. sp.; 261-9-4, 110-112, ×135.

?Stichomitra campi (Campbell and Clark); 261-12-2, top, $\times 135$.

Stichomitra cathara Foreman; 261-12-2, top, $\times 135$.

Diacanthocapsa sp. B; 261-12-2, top, $\times 135$.

?Lithostrobus erectus Tan Sin Hok; 261-10-1, 10-12, ×150.

?Lithostrobus erectus Tan Sin Hok; 261-12, CC, $\times 150$.

Stichocapsa wichmanni Tan Sin Hok; 261-16-1, 101-103, $\times 135$.

Stichomitra asymbatos Foreman; 261-9-4, 110-112, ×135.

Eucyrtidium vermiculatium $\mathrm{n}$. sp.; holotype, 261-12-2, top, $\times 135$.

Eucyrtidium sp. cf. E. thiensis Tan Sin Hok; 261-12-2, top, $\times 135$.

?Stichocapsa procera Hinde; 261-12-2, top, $\times 135$.

?Stichocapsa procera Hinde; 261-12-2, top, $\times 135$.

Dictyomitra brouweri var. $\alpha$ (Tan Sin Hok); 261-9, CC, $\times 135$.

Dictyomitra brouweri var. $\gamma$ (Tan Sin Hok); 261-9-4, 50-52, $\times 135$.

?Dictyomitra malleola Aliev; 261-16-1, 101-103, ×135.

Stichocapsa pseudodecora Tan Sin Hok; 261-9-3, 90-92, $\times 135$.

Lithomitra pseudopinguis Tan Sin Hok; 261-12-2, top, X135.

?Lithocampe pseudochrysalis var. $\alpha$ Tan Sin Hok; 261-9-4, 50-52, $\times 135$.

?Eucyrtis molengraaffi (Tan Sin Hok); 261-12-2, 11-12, ×135.

Dictyomitra lilyae Tan Sin Hok; 261-9, CC, ×135.

Dictyomitra pseudoscalaris (Tan Sin Hok); 261-9, CC, $\times 135$.

Dictyomitra excellens (Tan Sin Hok); 261-16-1, 101-103, ×135. 
PLATE 11
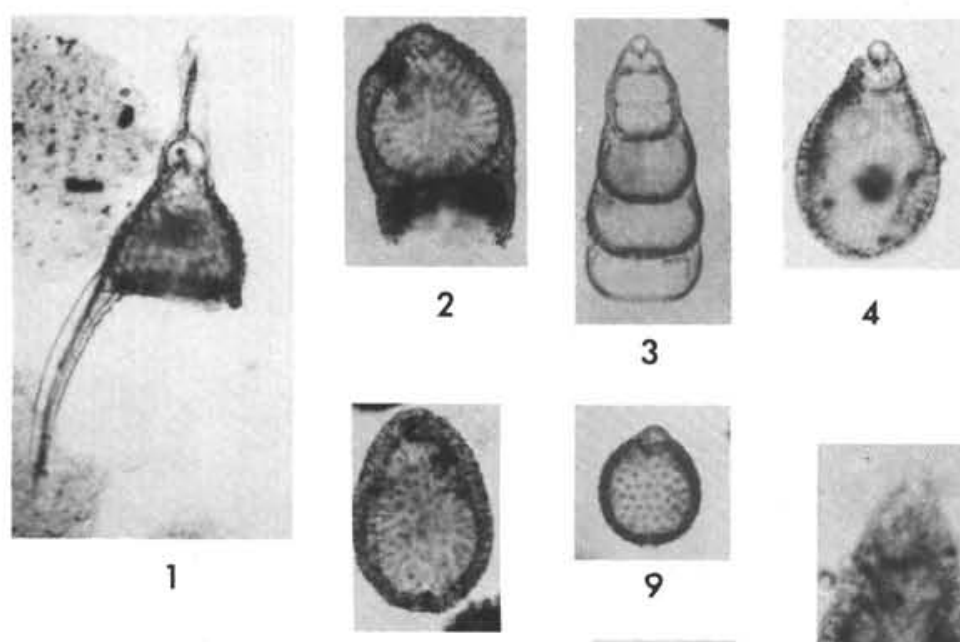

8
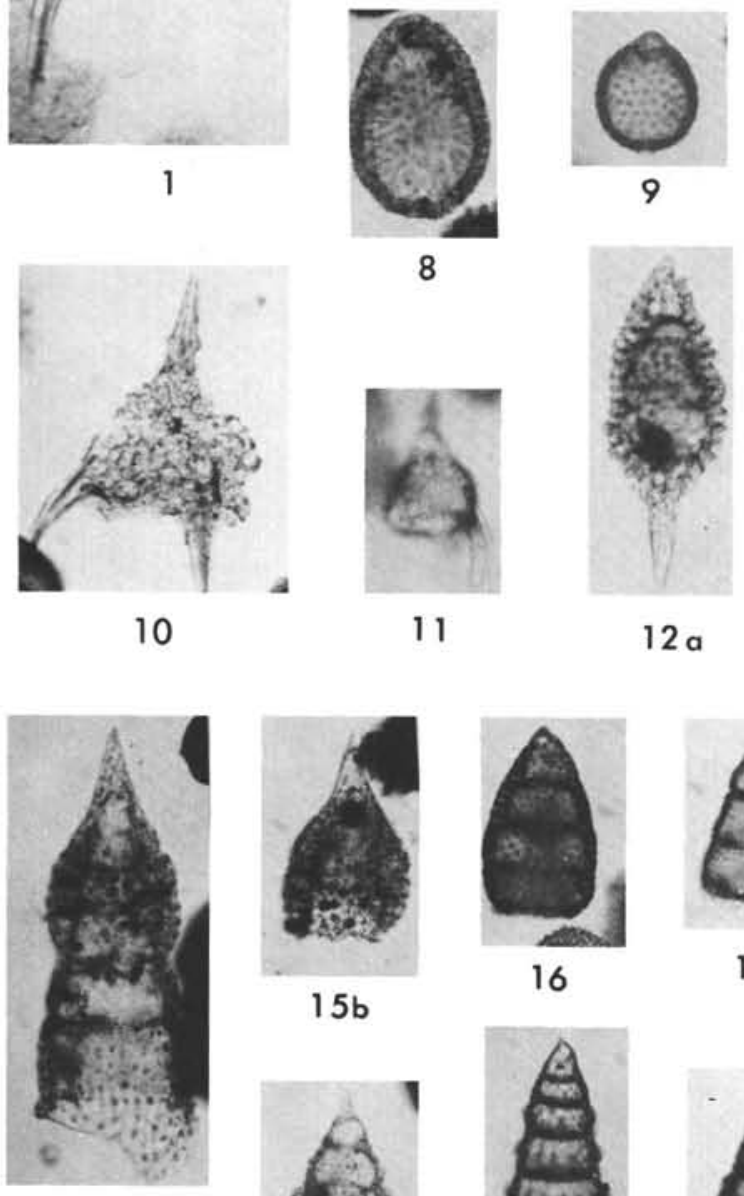

$15 a$

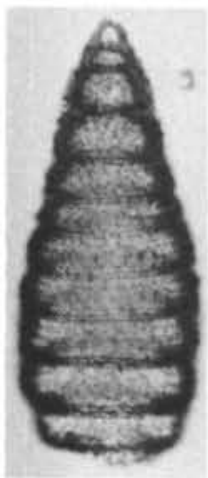

28
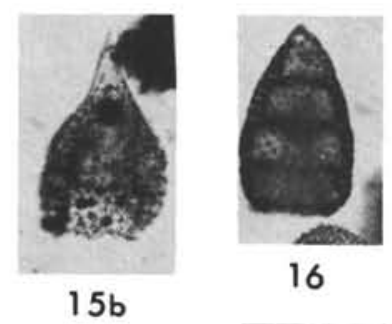

16

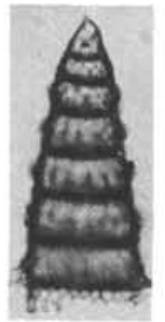

22

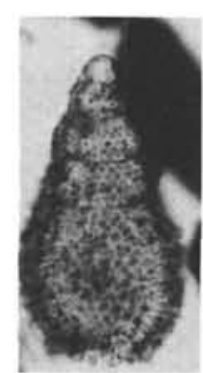

29

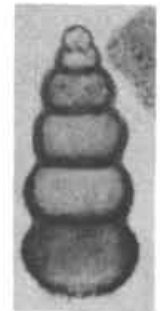

30
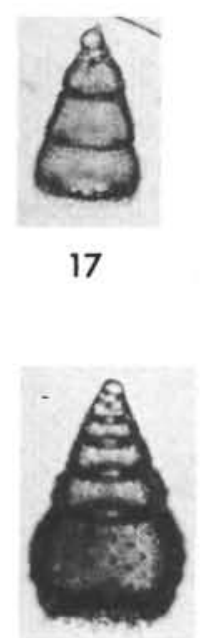

23

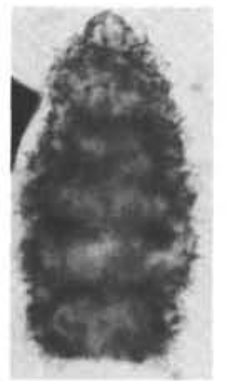

31

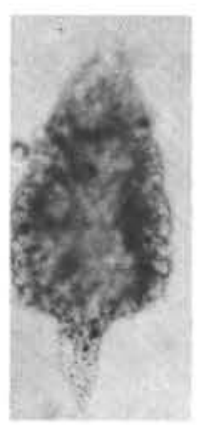

$12 b$

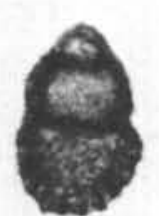

18

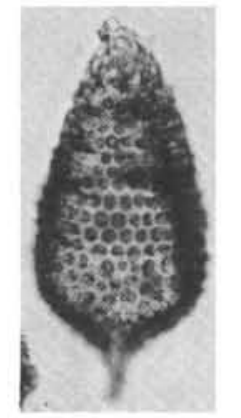

13

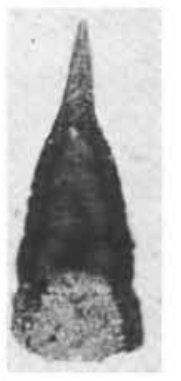

$19 a$

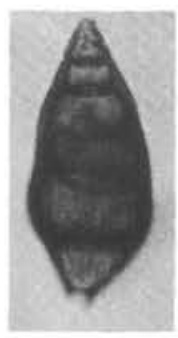

24

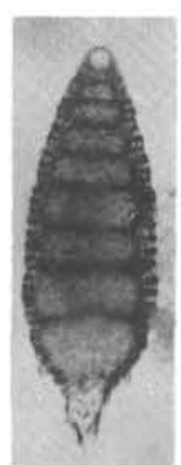

32

25
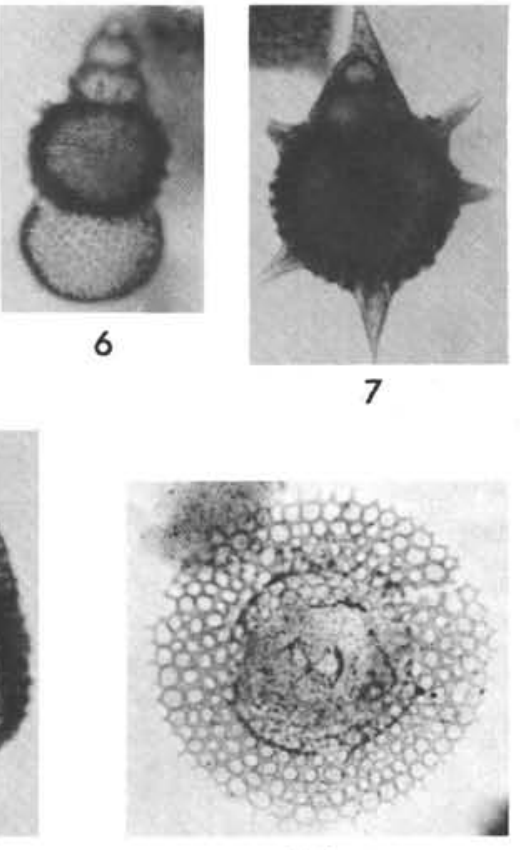

14
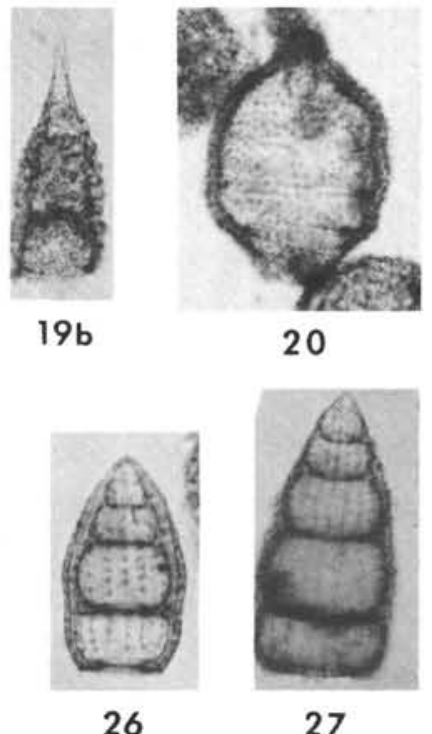

26

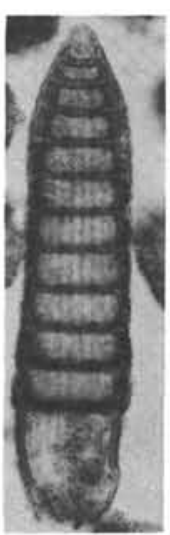

34 


\section{PLATE 12}

Figure 1 Amphipyndax (?) pyrgodes n. sp.; holotype, 261$12-2$, top, $\times 150$.

Figure 2 Amphipyndax (?) epiplatys n. sp.; holotype, 261-9-4, $110-112, \times 150$.

Figure 3 Amphipyndax mediocris (Tan Sin Hok); 261-12, $\mathrm{CC}, \times 150$.

Figures 4 a-c Bathropyramis timorensis n. sp. (a) apical view, 261-9-4, 110-112, $\times 135$. (b) sagittal view, holotype, 261-9-4, 110-112, $\times 135$. (c) sagittal view, $261-9, \mathrm{CC}, \times 150$.

Figure $5 \quad$ ?Hemicryptocapsa pseudopilula Tan Sin Hok; 2619-4, 110-112, ×150.

Figure 6 ?Cornutella californica var. A Campbell and Clark, emend. Foreman; 261-12-2, 11-12, $\times 150$.

Figure 7 ?Cornutella californica var. B Campbell and Clark, emend. Foreman; 261-12, CC, $\times 150$.

Figure 8 ?Cyrtocalpis operosa Tan Sin Hok; 261-15, CC, $\times 150$.

Figure $9 \quad$ Lophophaena sp.; 261-12-2, top, $\times 135$.

Figure $10 \quad$ Lophophaeniid sp. B; 261-16-1, 101-103, $\times 150$.

Figure $11 \quad$ Lophophaeniid sp. A; 261-12-2, top, $\times 150$.

Figure 12 Lophophaeniid sp. C; 261-12, CC, $\times 150$.

Figures 13 a-c Eucyrtis columbarius n. sp. (a) with bulbous terminal segment, 261-12-2, top, $\times 260$. (b) with apical horn, 261-12, CC, $\times 150$. (c) with terminal spines, holotype, 261-12-2, 11-12, $\times 150$.

Figures 14 a-d Lithocampe chenodes n. sp. (a) 261-12-2, top, $\times 425$. (b) ridges in meshwork, 261-12, CC, $\times 580$. (c) holotype, 261-12, CC, $\times 135$. (d) 261-12, CC, $\times 260$.

Figures 15a, b Eucyrtis bulbosus n. sp. (a) holotype, 261-12-2, 11$12, \times 330$. (b) $261-12-2,11-12, \times 150$.

Figures 16a, b Eucyrtis hanni (Tan Sin Hok), (a) 261-12-2, top, $\times 150$. (b) 261-12-2, top, $\times 330$. 
PLATE 12
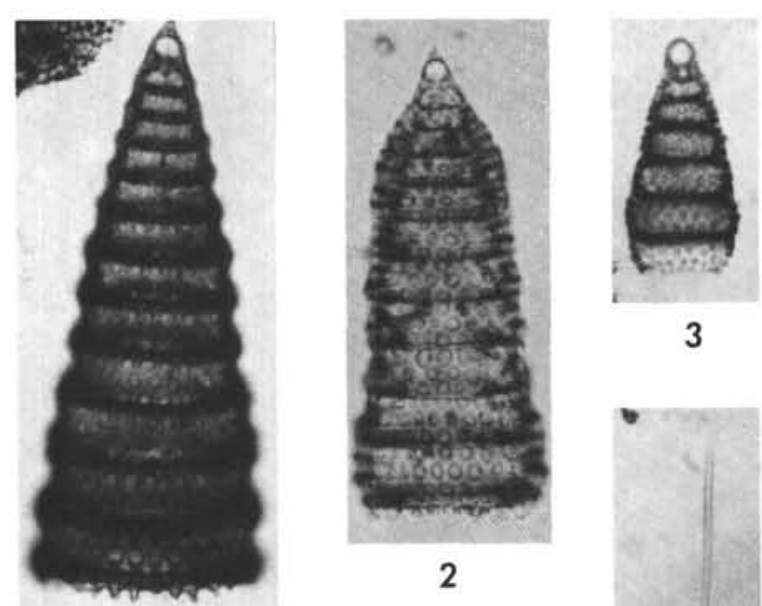

3
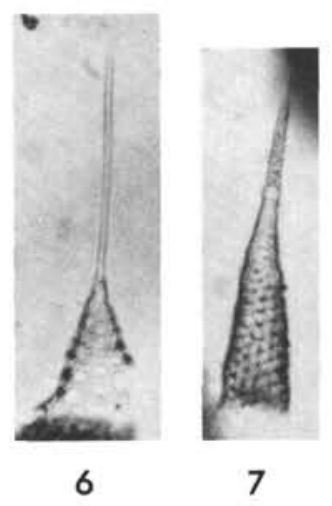

7

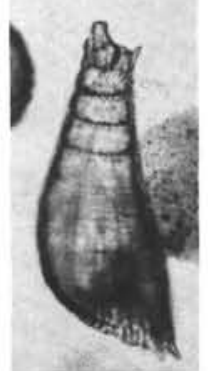

$13 b$

$13 a$

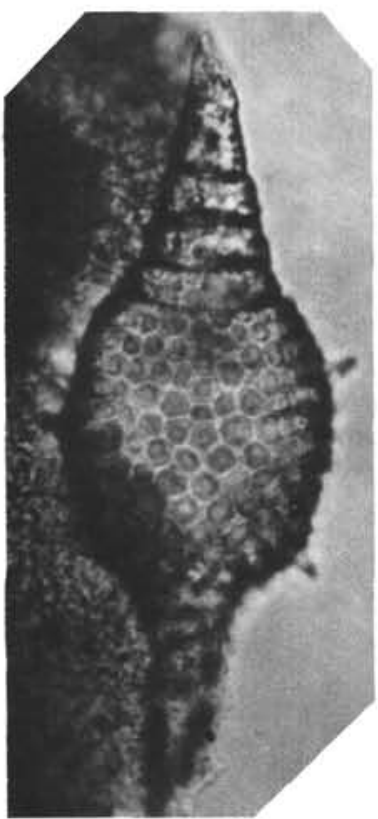

$15 a$
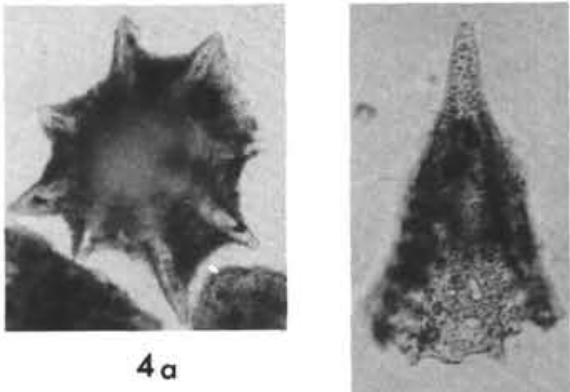

$4 \mathrm{~b}$
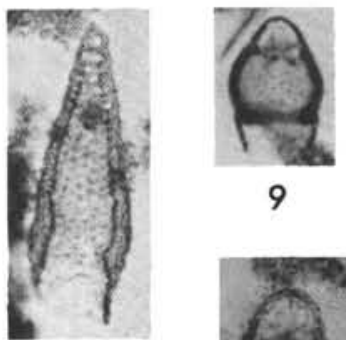

8
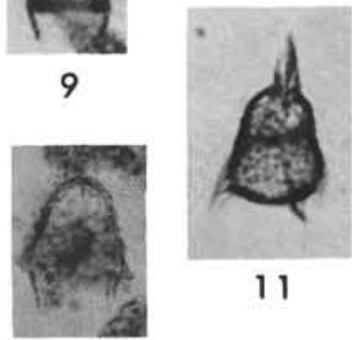

11

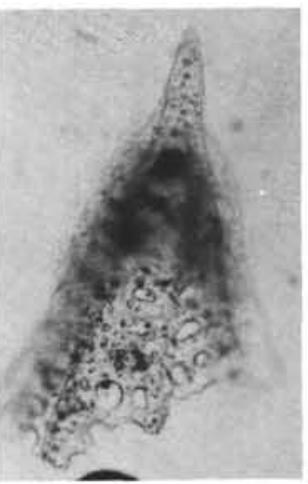

$4 c$

10

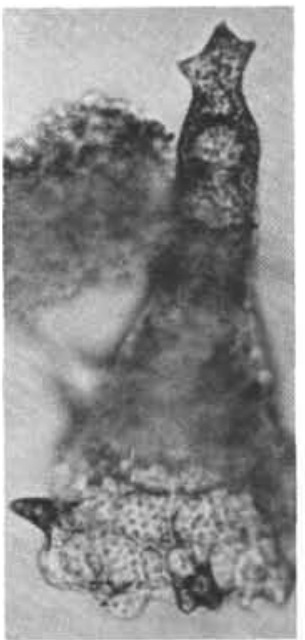

$14 a$

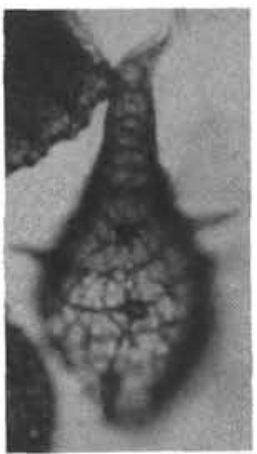

$14 c$

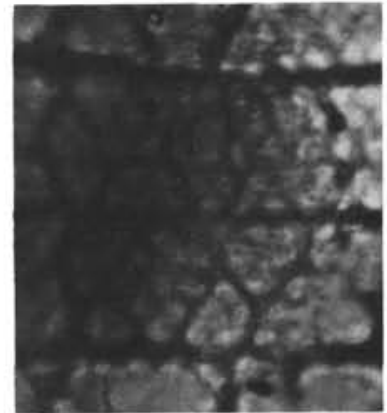

$14 b$

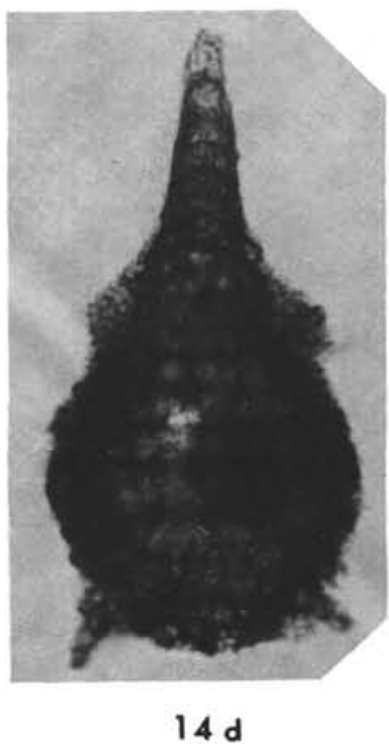


PLATE 13

(Magnification $\times 150$ )

Figure $1 \quad$ Heliodiscus echiniscus Haeckel; 261-1-4, 39-41.

Figure 2 Larcospira quadrangula Haeckel; 262-1-4, 39-41.

Figure $3 \quad$ Siphonosphaera sp. B; 261-1-2, 31-33.

Figure $4 \quad$ Polysolenia sp.; 262-1-2, 31-33.

Figure 5 Disolenia zanguebarica (Ehrenberg); 262-1-2, 89-

91.

Figure 6 Collosphaera tuberosa (Haeckel); 261-1-2, 31-33.

Figure 7 Hexapyle dodecantha Haeckel; 260-1-1, 17-19.

Figure 8 Disolenia quadrata (Ehrenberg); 262-1-2, 31-33.

Figure 9 Siphonosphaera polysiphonia Haeckel; 262-1-2, 8991.

Figure $10 \quad$ Siphonosphaera sp. A; 262-1-2, 31-33.

Figure $11 \quad$ Heliodiscus asteriscus Haeckel; 262-1-2, 31-33.

Figure 12 Pyloniid sp.; 262-1-2, 89-91.

Figure $13 \quad$ Pyloniid sp.; 262-1-2, 31-33.

Figure 14 Collosphaera polygona Haeckel; 262-7, CC.

Figure $15 \quad$ Otosphaera polymorpha Haeckel; 262-1-2, 89-91.

Figure $16 \quad$ Elatomma sp.; 262-3-2, 84-86.

Figure 17 Cladococcus scoparius Haeckel; 262-6, CC.

Figure 18 ?Cladococcus abietinus Haeckel; 262-1-2, 31-33.

Figure 19 ?Cladococcus pinetum Haeckel; 262-2, CC.

Figure $20 \quad$ Actinommid 1 gen. and sp. indet.; 260-1-1, 17-19. 
PLATE 13

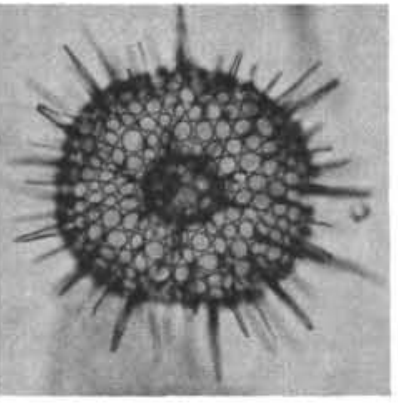

1

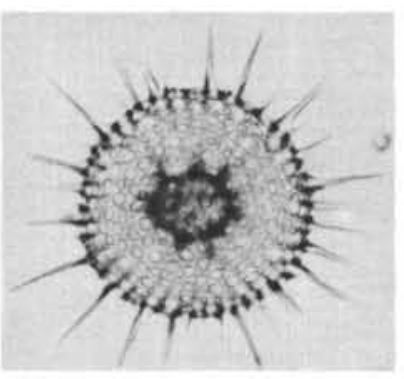

11

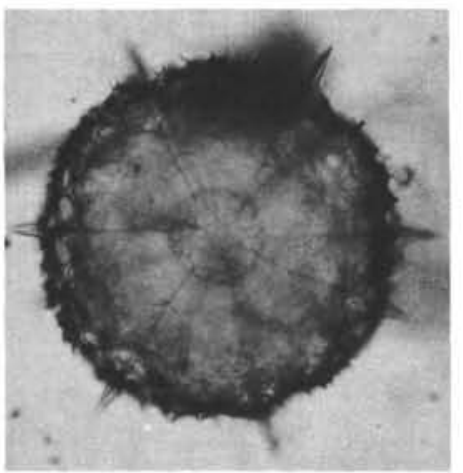

16

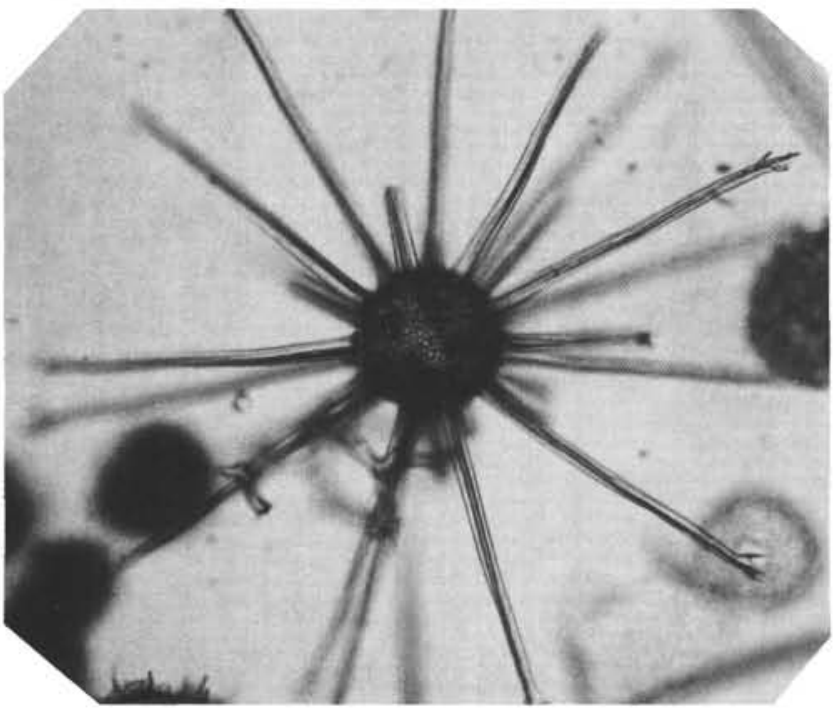

19

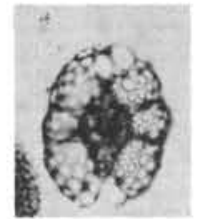

2

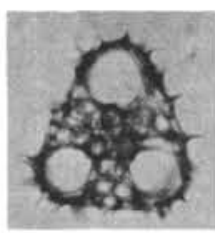

7

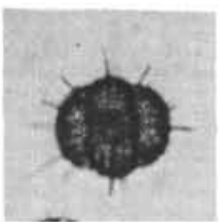

12

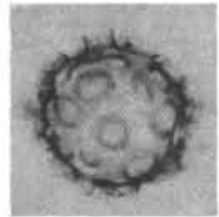

3

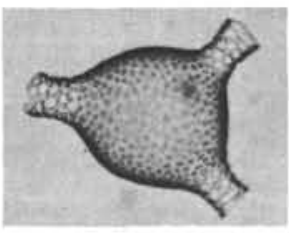

8

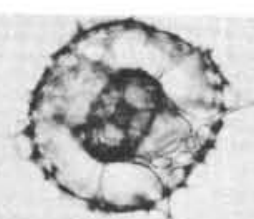

13

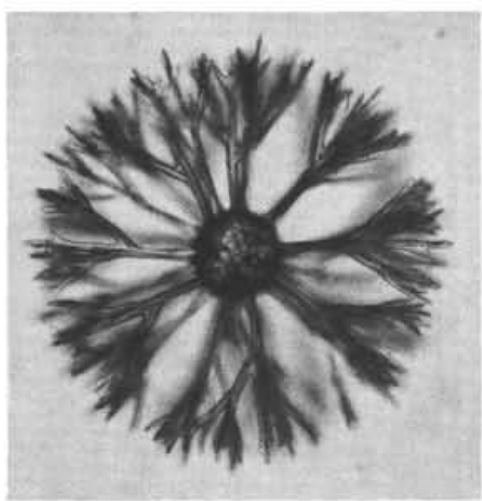

17
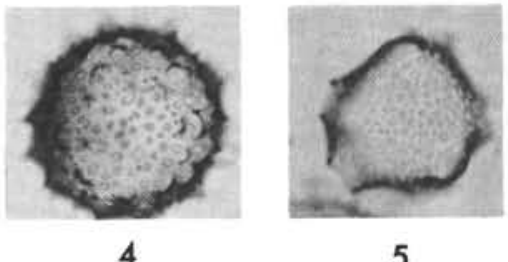

5
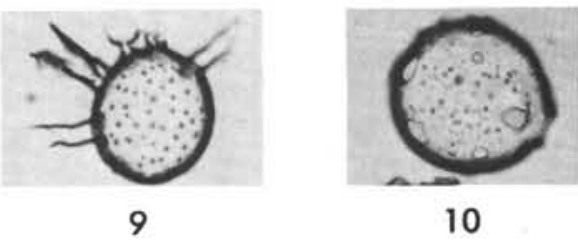

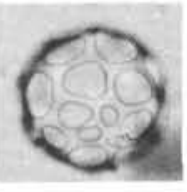

14
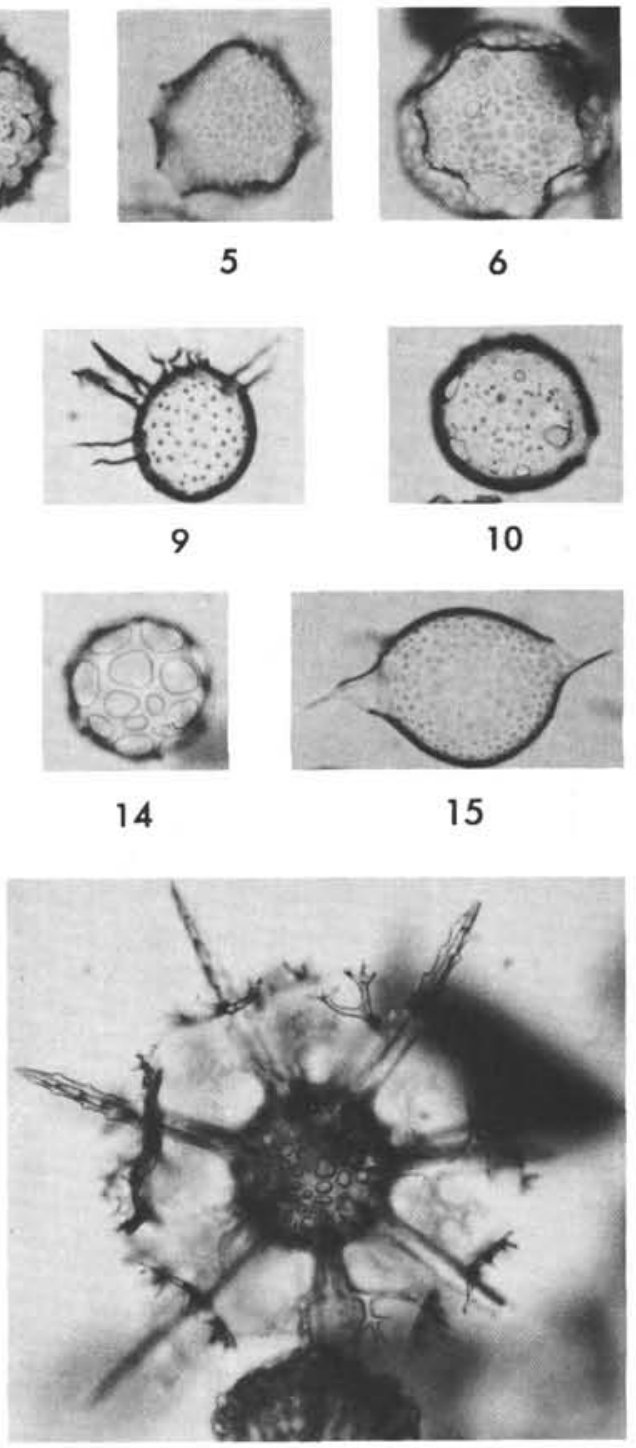

18

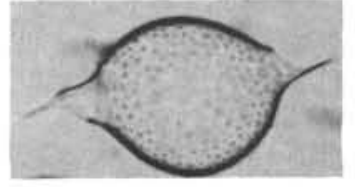

15

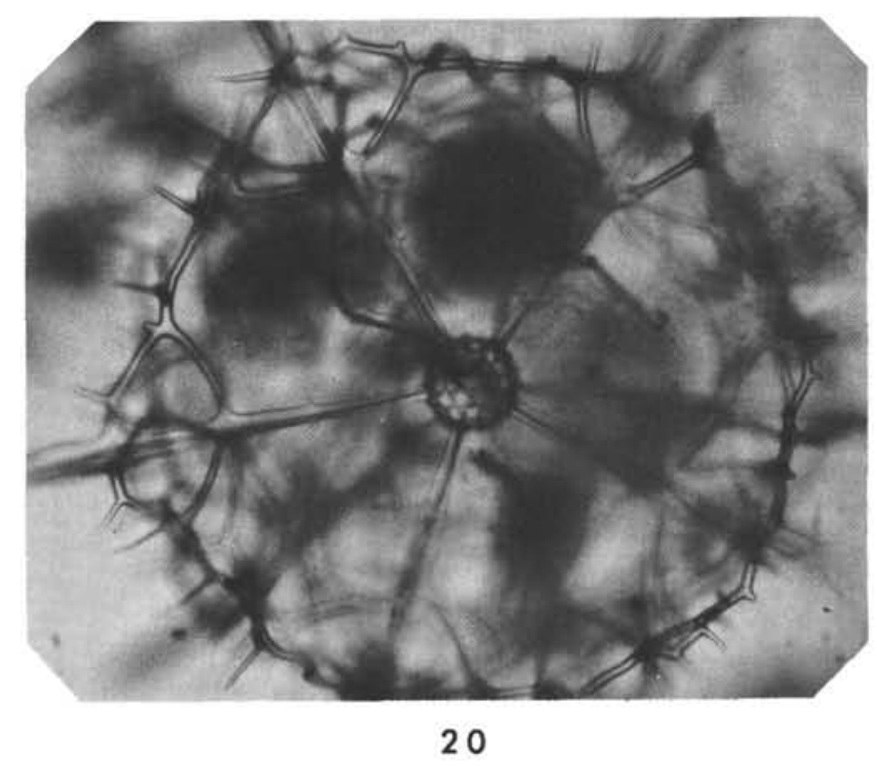


PLATE 14

(Magnification $\times 150)$

Figure 1 Astrosphaera hexagonalis Haeckel; 262-1-2, 89-91.

Figure 2 Actinommid 2 gen. and sp. indet.; 262-1-2, 31-33.

Figure 3 Saturnalis circularis Haeckel; 262-1-4, 39-41.

Figure $4 \quad$ Cladococcus sp.; 262-1-2, 89-91.

Figure 5 Ommatartus tetrathalamus (Haeckel); 262-1-2, 3133.

Figure $6 \quad$ Spongoliva ellipsoides Popofsky; 262-1-2, 89-91.

Figure 7 Stylosphaera sp.; 262-2-2, 94-96.

Figure $8 \quad$ Stylatractus universus Hays; 262-1-2, 31-33.

Figure 9 Actinommid 3 gen. and sp. indet.; 261-1, CC.

Figure 10 Actinomma arcadophorum Haeckel; 262-1-2, 31-33.

Figure 11 Haliomma erinaceum Haeckel; 262-1-2, 89-91.

Figure 12 Haliomma sp. cf. H. glisifra Renz; 262-1-2, 31-33.

Figure 13 Hexalonche sp.; 262-1, CC.

Figure 14 Centrocubus octostylus Haeckel; 262-1-2, 31-33.

Figure 15 Xiphosphaera tesseractis Dreyer; 262-7, CC.

Figure 16 Spongosphaera streptacantha Haeckel; 262-1-2, 8991. 


\section{PLATE 14}
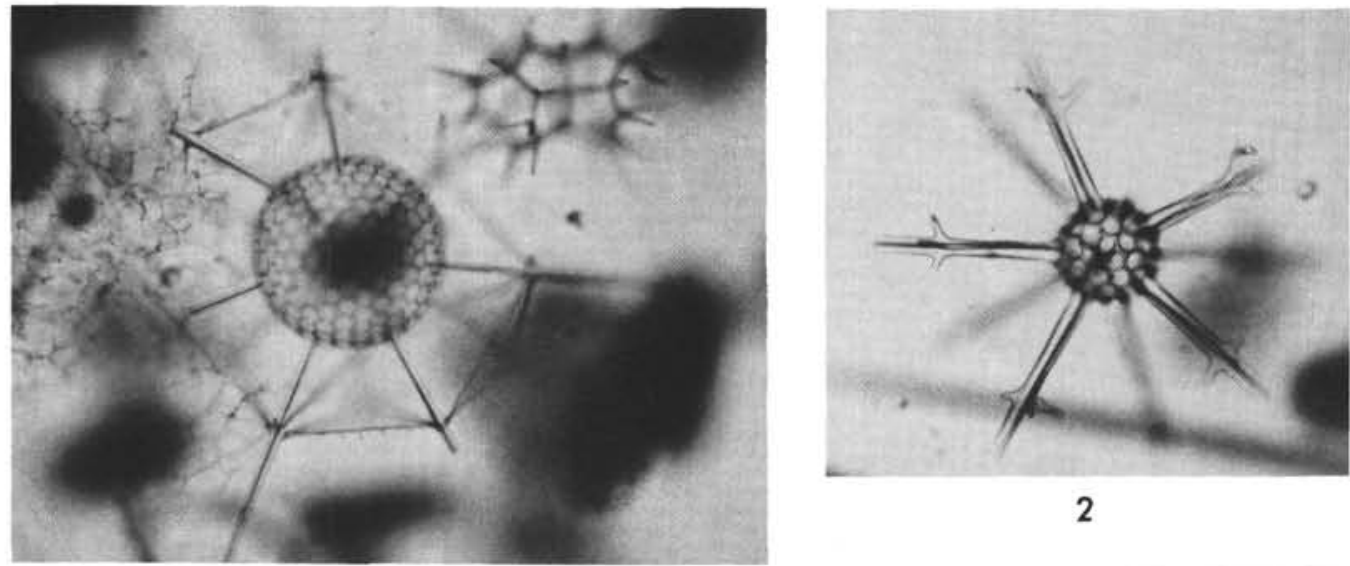

2

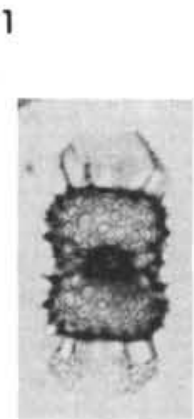

5

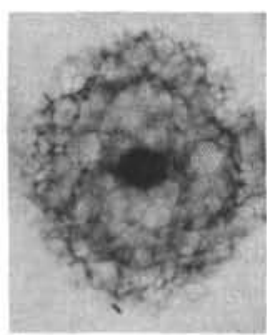

6

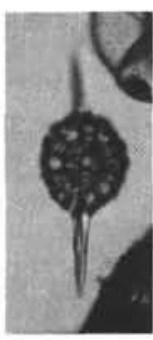

7

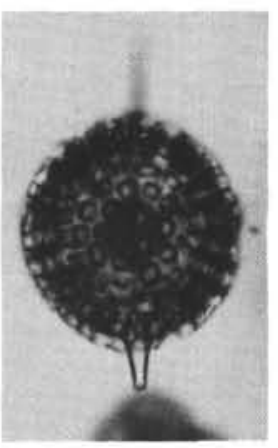

8

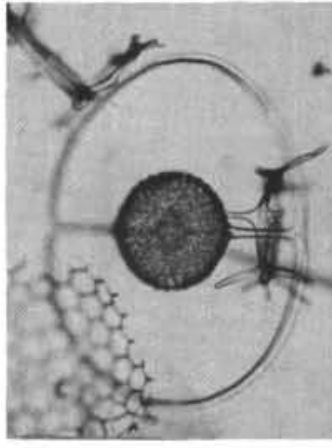

3

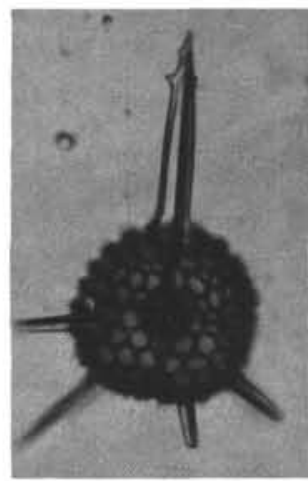

9

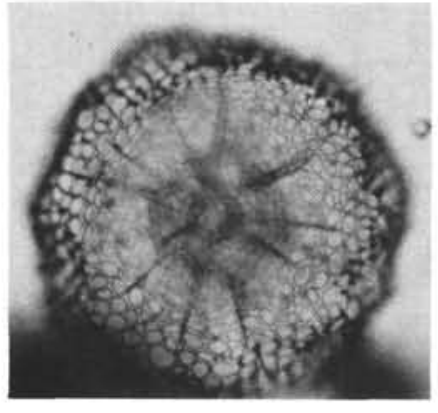

10

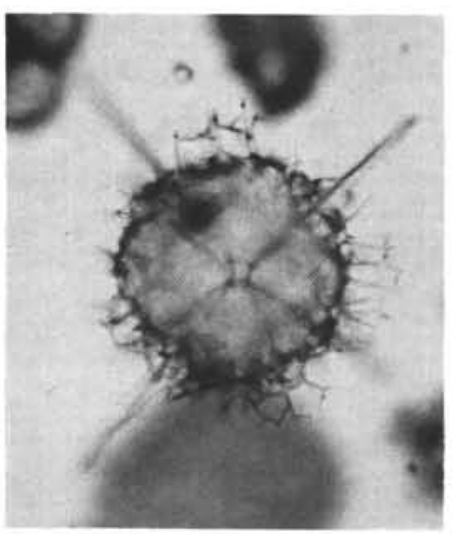

14

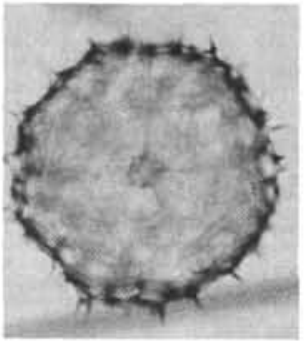

11

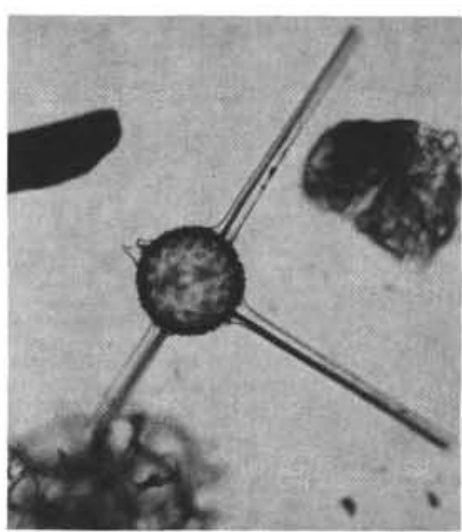

15
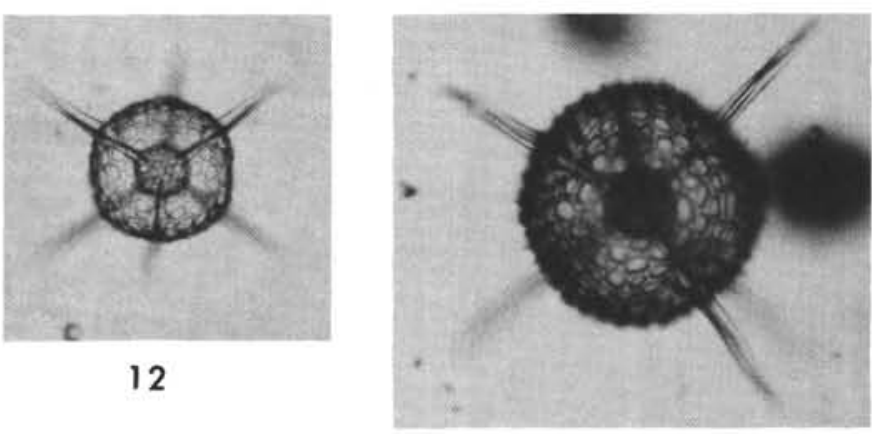

13

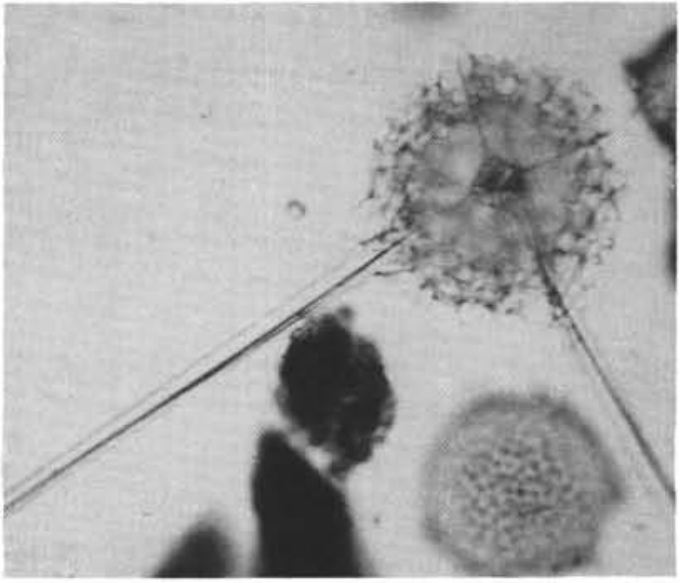

16 


\author{
PLATE 15 \\ (Magnification $\times 150$ )
}

Figure 1 Euchitonia mulleri Haeckel; 262-1-2, 31-33.

Figure $2 \quad$ Rhopalastrum sp. 1; 262-1-2, 89-91.

Figure $3 \quad$ Rhopalastrum sp. 2; 262-1-2, 31-33.

Figure 4 Spongocore diplocylindrica Haeckel; 262-1-2, 8991.

Figure $5 \quad$ Spongolena sp.; 262-3-2, 84-86.

Figure 6 Myelastrinae subfamily group; 262-1-2, 89-91.

Figure 7 Dictyocoryne profunda Ehrenberg; 262-1-2, 89-91.

Figure 8 Spongaster tetras Ehrenberg; 262-1-2, 31-33.

Figure 9 Spongotrochus multispinus Haeckel; 262-1-2, 3133.

Figure 10 Spongobrachium sp. aff. S. ellipticum Haeckel; 262-1, CC.

Figure $11 \quad$ Spongodiscus sp. 3; 262-1-2, 89-91.

Figure 12 Stylodictya sp. aff. S. multispina Haeckel; 262-1-2, 31-33.

Figure 13 Styptosphaera spongiacea Haeckel; 262-1-2, 31-33.

Figure 14 Amphirhopalum ypsilon Haeckel; 262-2-2, 94-96.

Figure 15 Tessarastrum straussii Haeckel; 260-1-1, 17-19.

Figure 16 Porodiscus microporus (Stöhr); 262-1-4, 39-41.

Figure 17 Stylochlamydium sp. aff. S. venustum (Bailey); 262$2, \mathrm{CC}$. 
PLATE 15
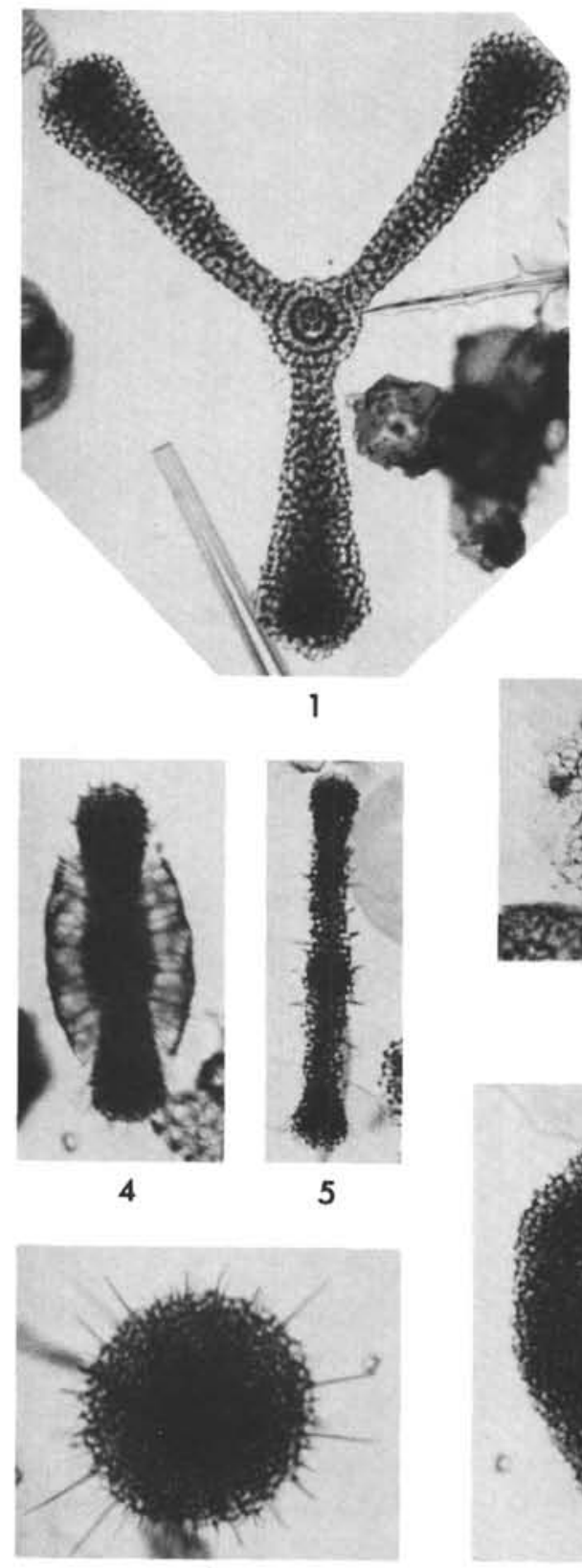

9

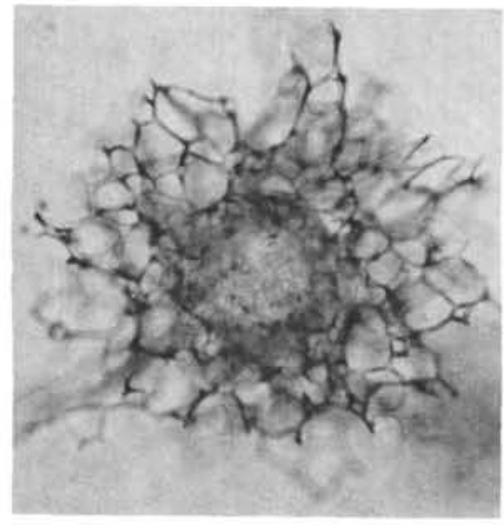

13

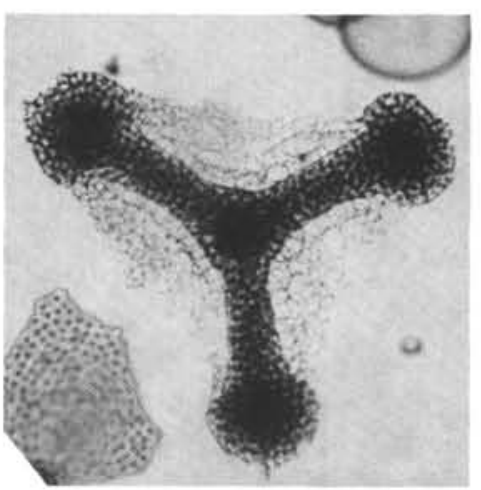

2
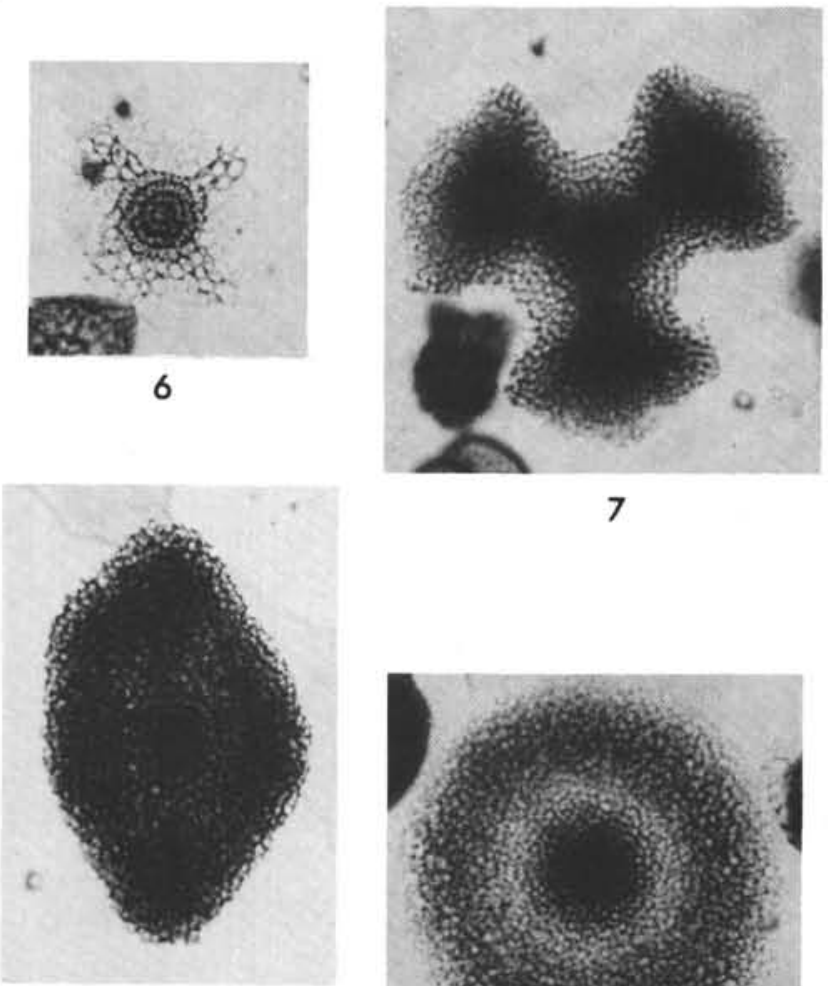

10
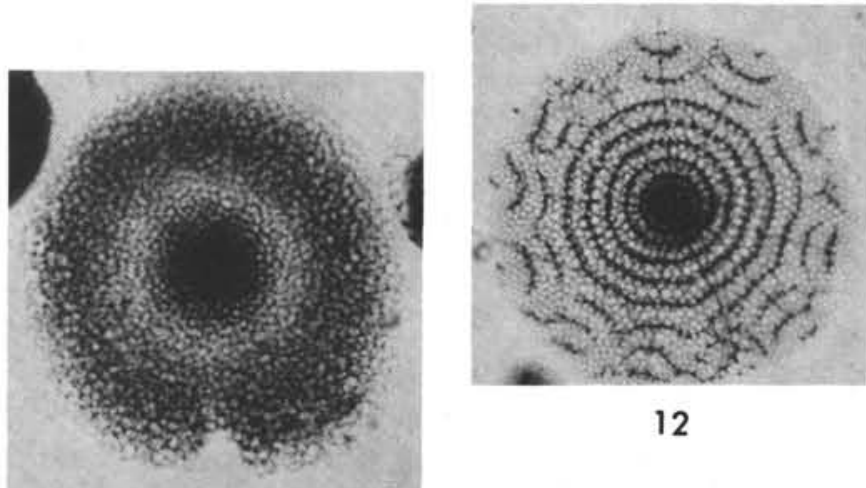

12

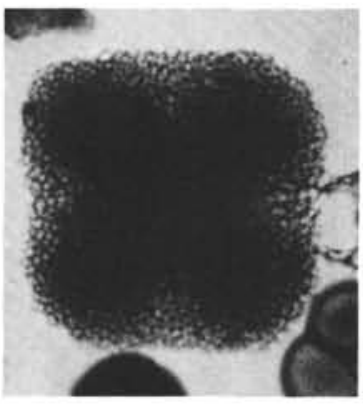

8

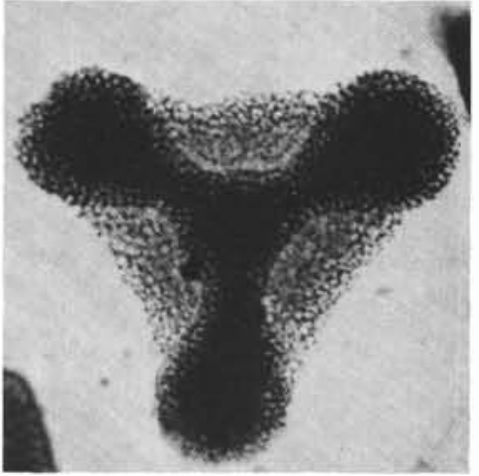

3

11

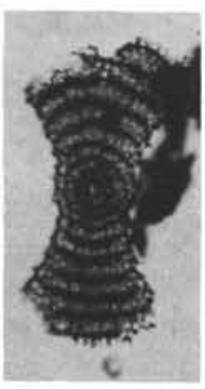

14

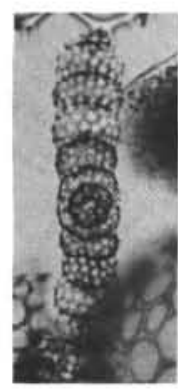

15

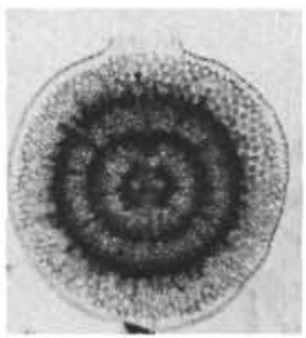

16

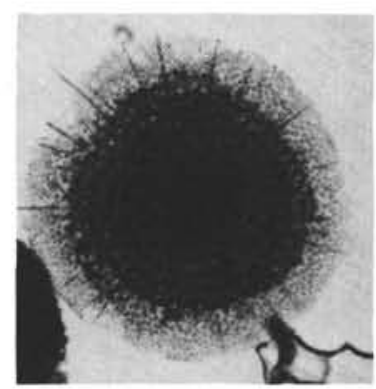

17 


\author{
PLATE 16 \\ (Magnification $\times 150$ )
}

Figure 1

Figure 2

Figure 3

Figure 4

Figure 5

Figure 6

Figure 7

Figure 8

Figure 9

Figure 10

Figure 11

Figure 12

Figure 13

Figure 14

Figure 15

Figure 16

Figure 17

Figure 18

Figure 19

Figure 20

Figure 21

Figure 22

Figure 23

Figure 24

Figure 25

Figure 26

Figure 27

Figure 28

Figure 29
Dictyocodon palladius Haeckel; 262-1-2, 89-91.

Cyrtopera sp. aff. C. languncula Haeckel; 262-1-2, 89-91.

Eucyrtidium hexagonatum Haeckel; 262-1-2, 89-91. Lithocampe sp.; 260-1-1, 17-19.

Sethoconus myxobrachia Strelkov and Reshetnyak; 260-1-1, 60-62.

Eucyrtidium hexastichum (Haeckel); 262-1-2, 8991.

?Eusyringium siphonostoma Haeckel; 262-1-2, 31$33.2 / 3$

Eucyrtidium acuminatum (Ehrenberg); 262-1-2, 89 91.

Lampromitra parabolica Popofsky; 262-1-2, 89-91.

Corocalyptra kruegeri Popofsky; 262-1-2, 89-91.

Corocalyptra sp. aff. C. kruegeri Popofsky; 262-6, CC.

Lithopilium sphaerocephalum Popofsky; 262-6, CC.

Artopilium undulatum Popofsky; 262-2-2, 94-96.

Udan undulata Renz; 260-1-1, 17-19.

Eucyrtid 1 gen. and sp. indet.; 261-1, CC.

Clathrocyclas semeles Haeckel; 262-1, CC.

Theopilium pyramidale Popofsky; 262-1-2, 89-91.

Theocorys veneris Haeckel; 262-1-4, 39-41.

Lithostrobus hexagonalis Haeckel; 262-1-2, 89-91.

Eucyrtidium anomalum Haeckel; 262-1-2, 89-91.

Theocalyptra sp.; 262-1-2, 31-33.

Corocalyptra cervus (Ehrenberg); 262-1-2, 89-91.

Eucyrtid 3 gen.and sp. indet.; 262-1-2, 89-91.

Stichopilium bicorne Haeckel; 262-1-2, 89-91.

Calocyclas monumentum Haeckel; 262-1-2, 89-91.

Nassellarian gen. and sp. indet.; 262-1-2, 89-91.

?Pteropilium stratiotes Haeckel; 262-1-2, 89-91.

Lithopera bacca Ehrenberg; 262-1-2, 89-91.

?Cyclampterium neatum Sanfilippo and Riedel; 260-1-3, 25-27. 
PLATE 16

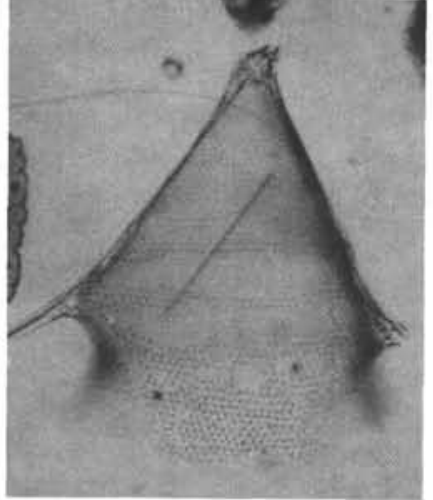

1
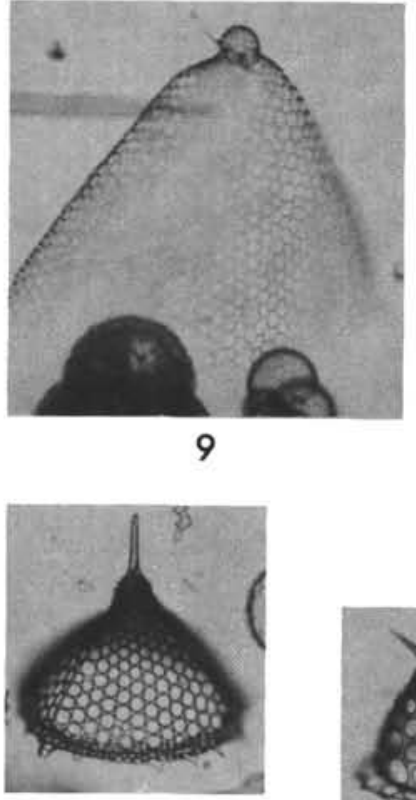

15

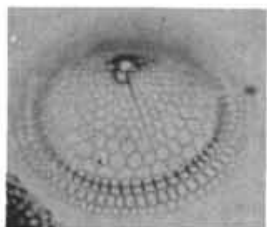

21

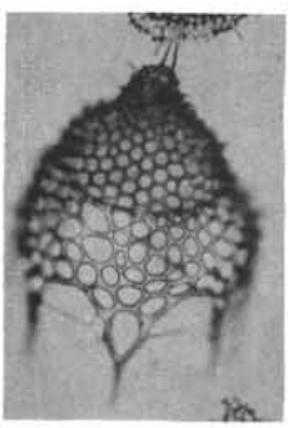

25

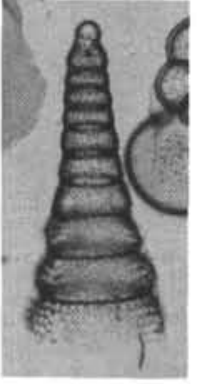

2

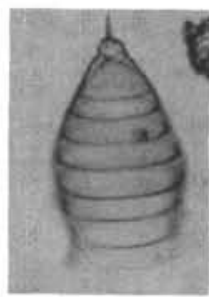

6

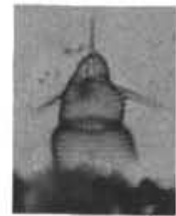

10

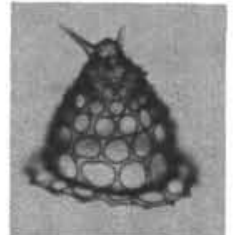

16

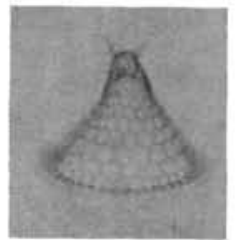

22

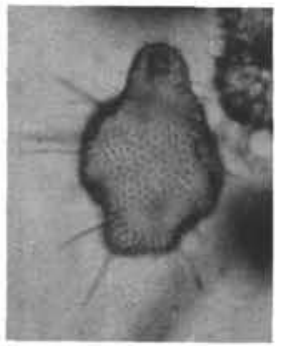

26

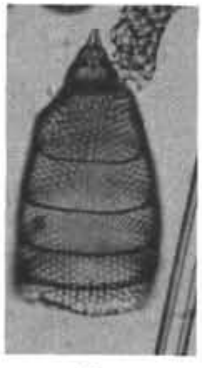

3

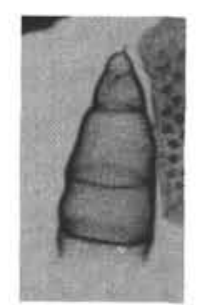

7

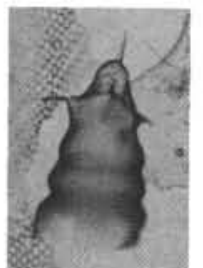

11

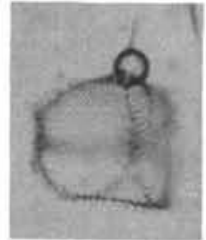

17

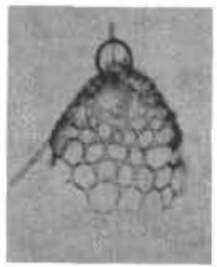

23

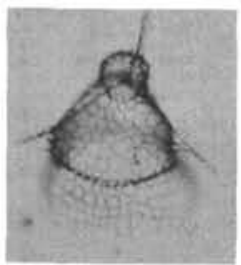

27
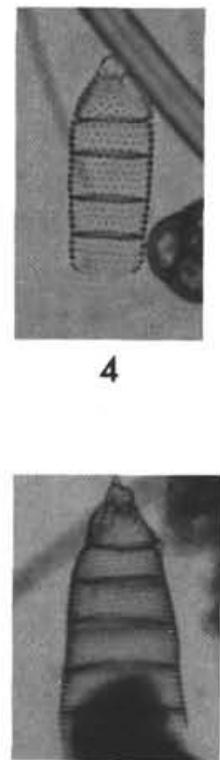

8

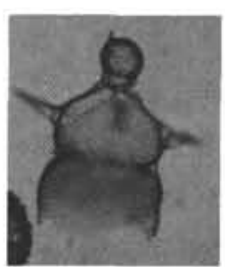

12

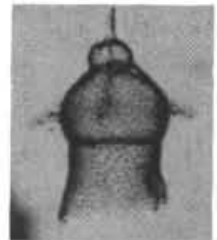

18

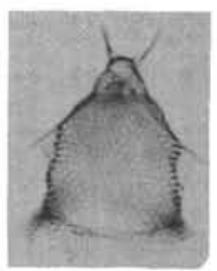

24

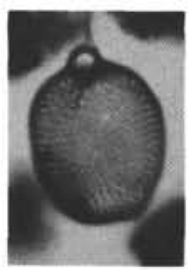

28

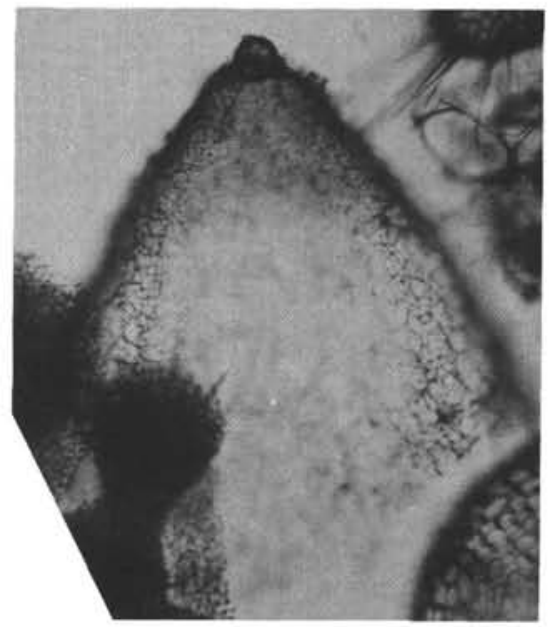

5

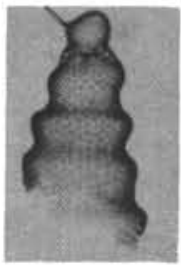

13

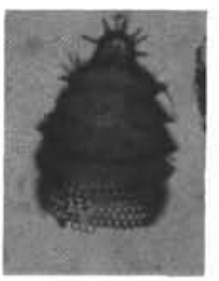

19
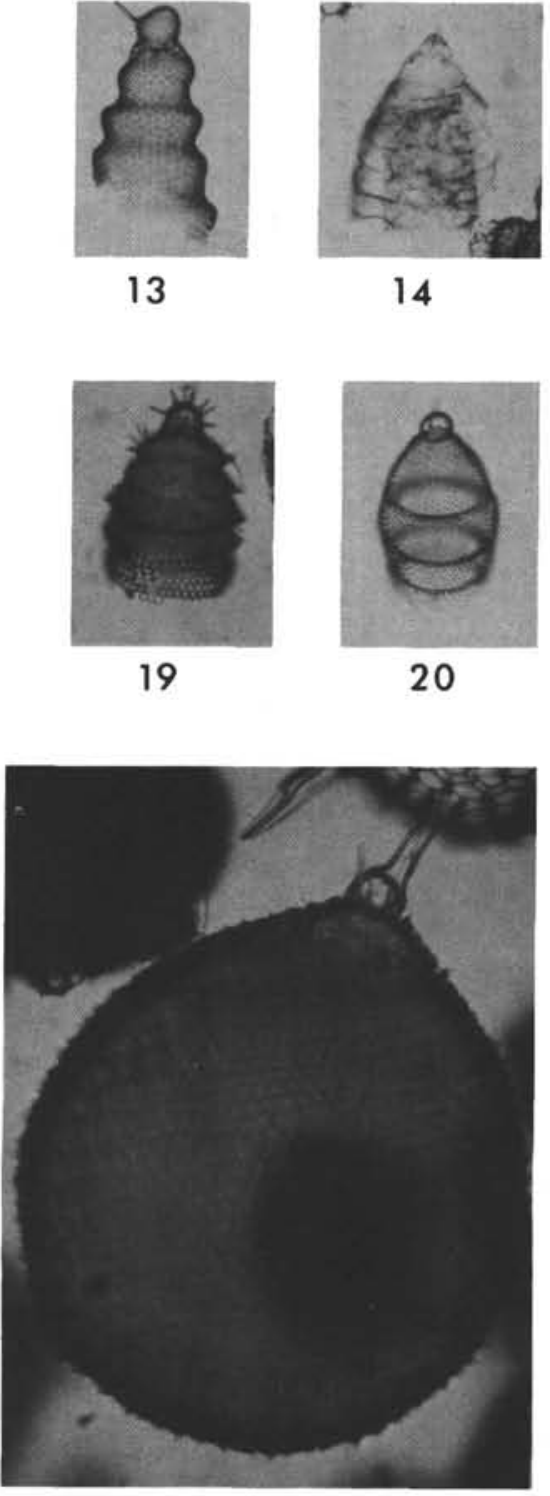

29 
PLATE 17

(Magnification $\times 150)$

Figure 1 Eucyrtid 2 gen. and sp. indet.; 262-6, CC.

Figure 2 Dictyophimus crisiae Ehrenberg; 262-1-2, 89-91.

Figure 3 Stichocampe bironec Renz; 262-7, CC.

Figure $4 \quad$ Artopilium sp. aff. A. elegans Haeckel; 262-1-2, 8991.

Figure 5 Dictyoceras virchowii Haeckel; 262-1-2, 89-91.

Figure 6 Pterocanium bicorne Haeckel; 260-1-1, 17-19.

Figure 7 Pterocanium trilobum (Haeckel); 262-1-2, 31-33.

Figure $8 \quad$ Pterocanium praetextum (Ehrenberg); 262-1-2, 8991.

Figure 9 Pterocanium orcinum Haeckel; 260-1-3, 25-27.

Figure $10 \quad$ Corocalyptra killmari Renz; 262-14, CC.

Figure 11 a, b Archipilium sp. aff. A. macropus (Haeckel), (a) 260-1-1, 17-19. (b) apical view, 260-1-1, 17-19.

Figure 12 a, b Lithomitra lineata (Ehrenberg). (a) 262-2-2, 94-96. (b) 262-2-2, 94-96.

Figure 13 Artostrobium auritum (Ehrenberg); 262-1-4, 39-41.

Figure 14 Siphocampe corbula (Harting); 262-1-2, 31-33.

Figure $15 \quad$ Spirocyrtis sp. aff. S. scalaris Haeckel; 262-1-2, 8991.

Figure 16 Spirocyrtis scalaris Haeckel; 262-1-2, 89-91.

Figure 17 Carpocanistrum sp.; 262-1-2, 89-91.

Figure 18 Dictyophimus ? sp. indet.; 262-3-2, 84-86.

Figure 19 Litharachnium tentorium Haeckel; 262-1-2, 89-91.

Figure $20 \quad$ Cornutella sp. aff. C. longisetta Ehrenberg; 261-1, CC.

Figure 21 Carpocanarium papillosum (Ehrenberg); 262-3-2, 84-86.

Figure $22 \quad$ Artostrobium miralestense (Campbell and Clark); 262-1-2, 89-91.

Figure 23 Cinclopyramis infundibulum Haeckel; 262-3-2, 8486.

Figure 24 Cornutella profunda var. a Ehrenberg; 262-2, CC.

Figure 25 Cornutella profunda var. b Ehrenberg; 262-2-2, 94 96.

Figure 26 Bathropyramis sp.; 260-1-1, 17-19.

Figure 27 Bathropyramis ramosa Haeckel; 262-2, CC. 
PLATE 17

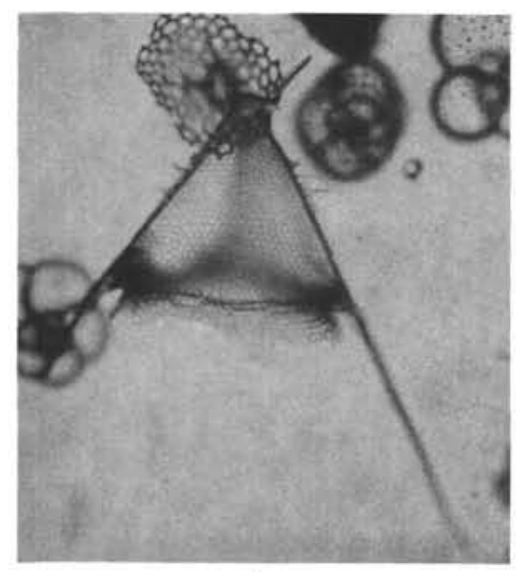

1

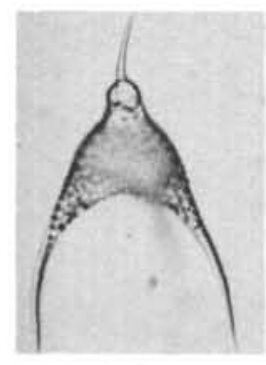

5

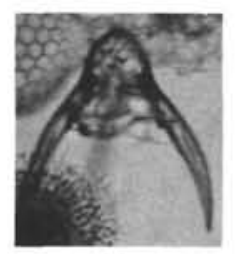

$11 a$
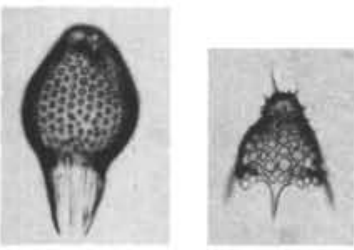

17

18

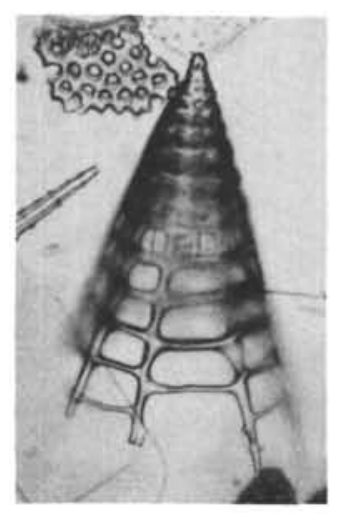

23

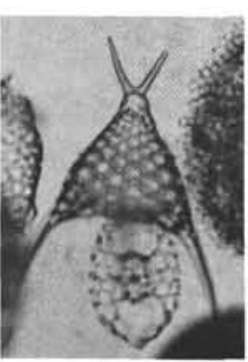

6

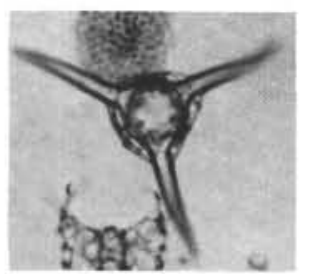

$11 \mathrm{~b}$

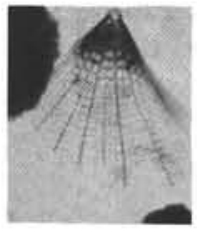

19

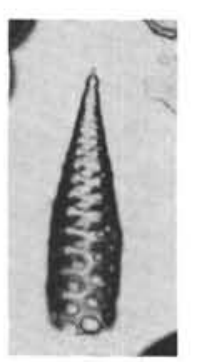

24

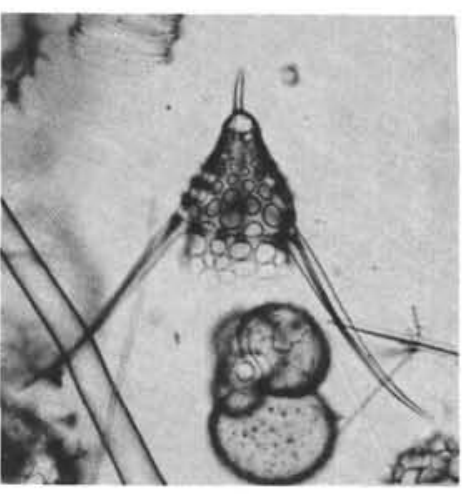

2

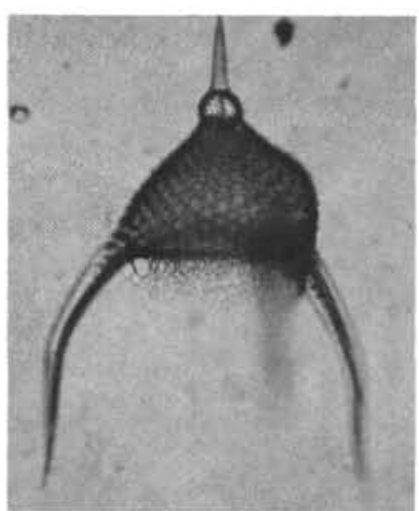

7

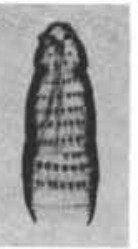

12
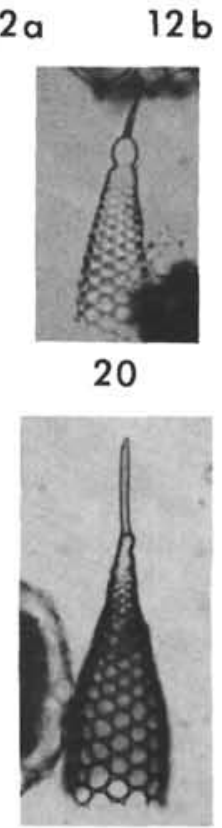

25
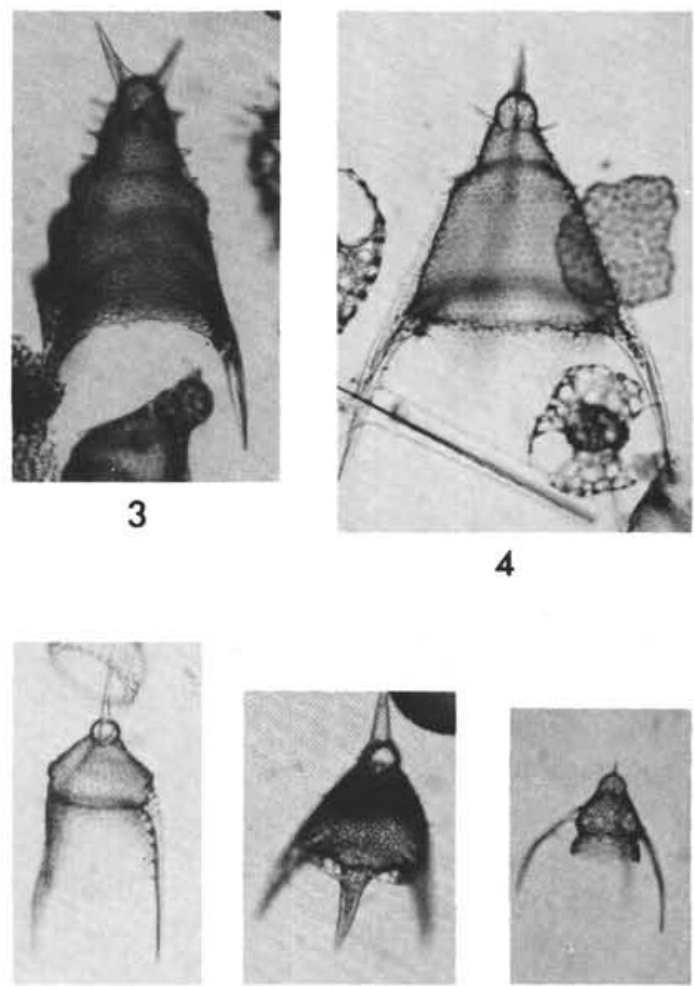

8
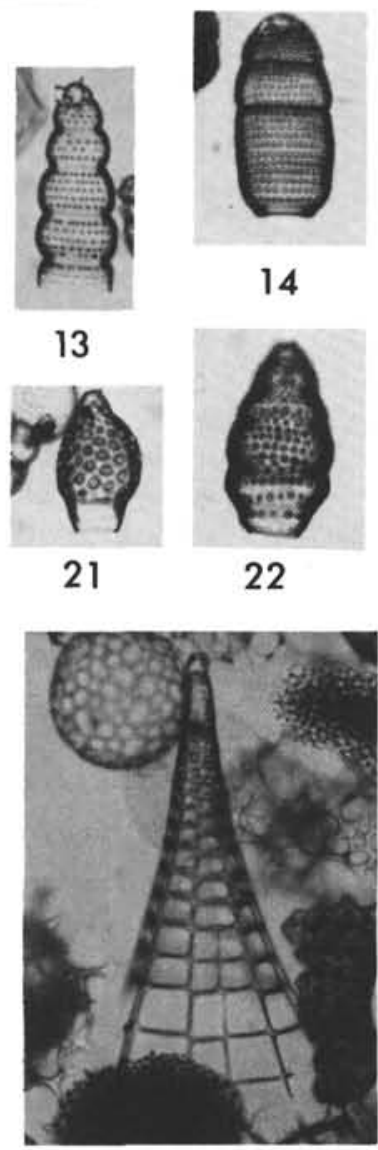

26

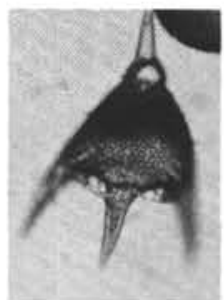

9

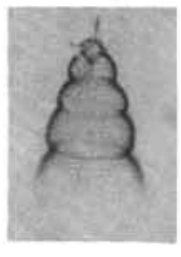

15
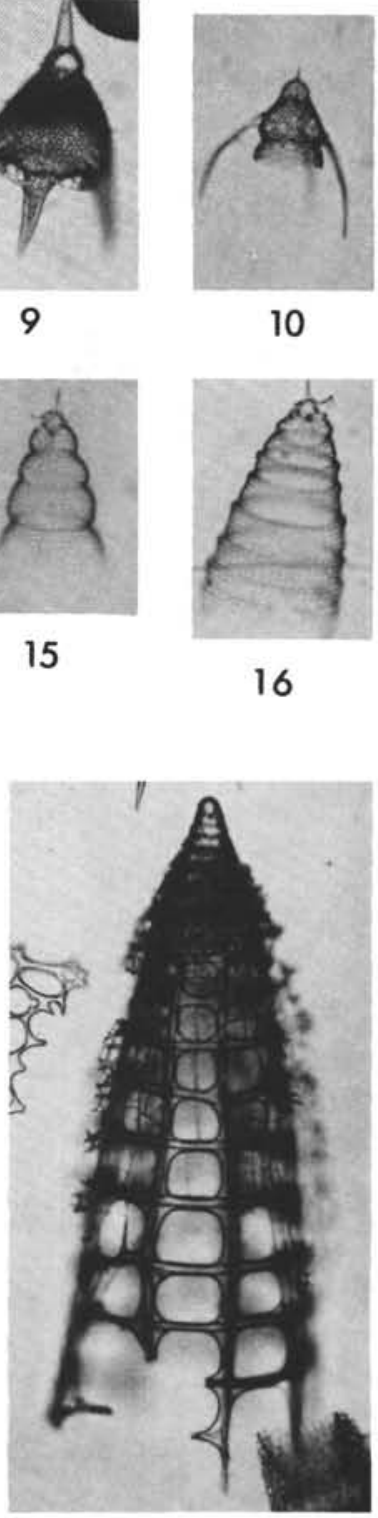

27 
PLATE 18

(Magnification $\times 150$ )

Figure $1 \quad$ Verticillata hexacantha Popofsky; 262-2-2, 94-96.

Figure 2 Pseudodictyophimus gracilipes (Bailey) tetracanthus (Popofsky); 262-1-2, 89-91.

Figure 3 Clathrocanium sp.; 262-1-2, 89-91.

Figure $4 \quad$ Clathrocorys sp.; 262-1-2, 89-91.

Figure $5 \quad$ Callimitra sp.; 262-1-4, 39-41.

Figure $6 \quad$ Lophophaena cyclindrica (Cleve); 262-2-2, 94-96.

Figure 7 Peromelissa phalacra (Haeckel), emend. Petrushevskaya; 262-1-4, 39-41.

Figure 8 Psilomelissa calvata Haeckel; 262-3-2, 84-86.

Figure 9 Acrobotrys sp.; 262-1-2, 89-91.

Figure 10 Botryopyle dictyocephalus Haeckel; 262-3-2, 84-86.

Figure 11 Dictyophimus tetracanthus Popofsky; 262-1-2, 8991.

Figure 12 ?Amphiplecta acrostoma Haeckel; 262-1-2, 89-91.

Figure 13 Lophophaenoma sp. aff. L. witjazii Petrushevskaya; 262-1-2, 31-33.

Figure 14 Lithomelissa monoceras Popofsky; 262-1-2, 89-91.

Figure $15 \quad$ Botryocyrtis sp.; 262-1-2, 89-91.

Figure 16 Centrobotrys thermophila Petrushevskaya; 262-2-2, 94-96.

Figure 17 Sethophormis rotula Haeckel; 262-1-2, 89-91.

Figure 18 a, b Sethophormis pentalactis Haeckel; (a) 262-1-2, 8991. (b) 262-1, CC.

Figure 19 Botryocyrtis quinaria Ehrenberg; 262-2-2, 94-96.

Figure 20 Botryopyle dictyocephalus Haeckel; 262-1-2, 89-91.

Figure 21 Sethophormis sp.; 262-1-2, 31-33.

Figure 22 Sethophormis $\mathrm{sp}$. aff. S. pentalactis Haeckel; 262-22, 94-96.

Figure 23 Lamprotripus butschlii (Haeckel); 262-1-2, 89-91.

Figure 24 Theophormis callipilium Haeckel; 262-4, CC. 
PLATE 18

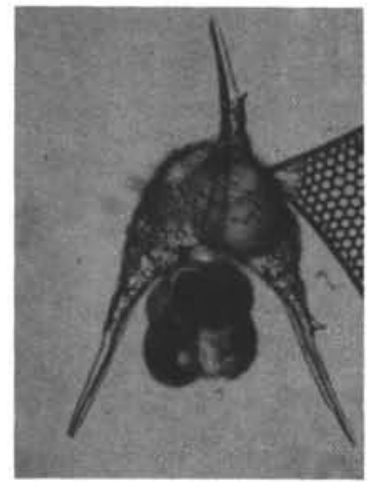

1

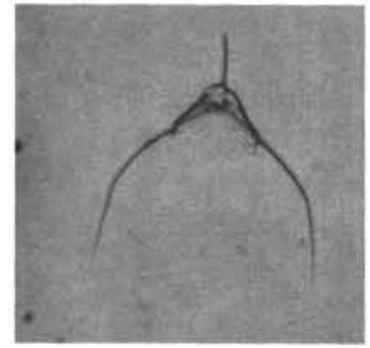

11

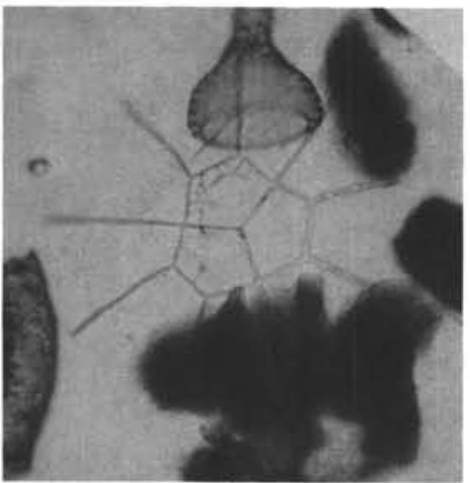

17

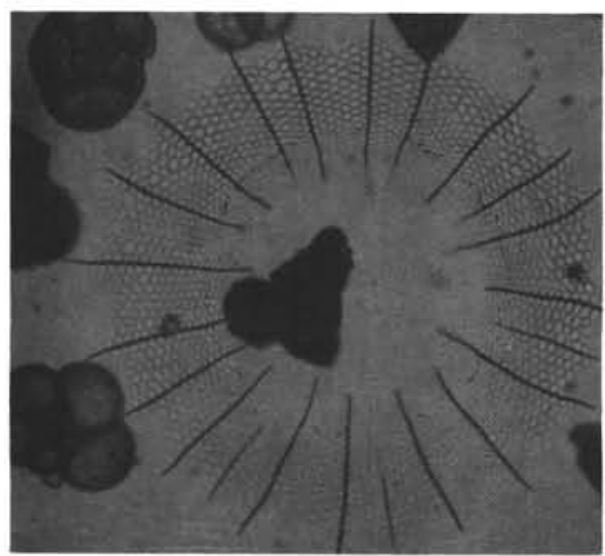

21
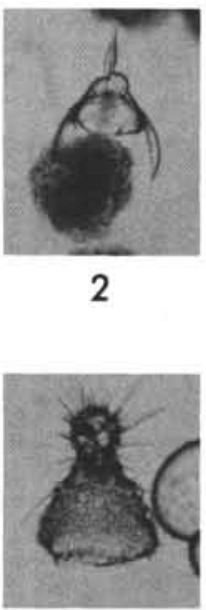

6

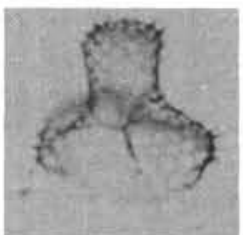

12

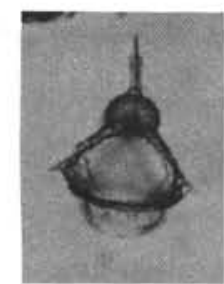

3

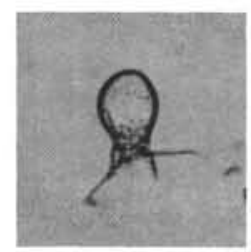

7

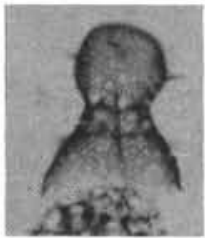

13

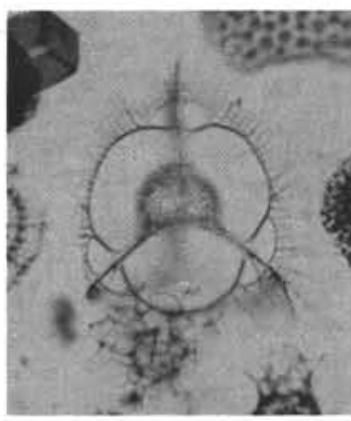

4

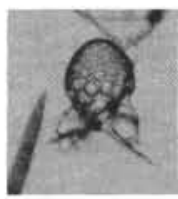

8

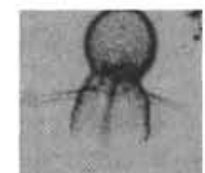

14

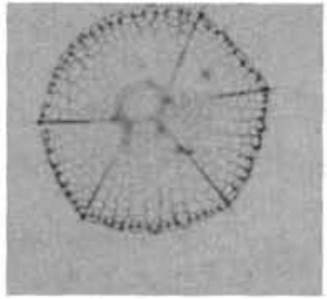

$18 a$

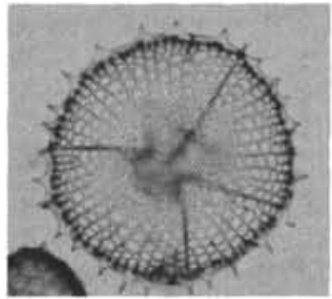

$18 \mathrm{~b}$

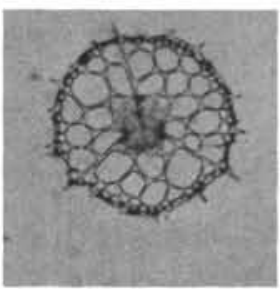

22

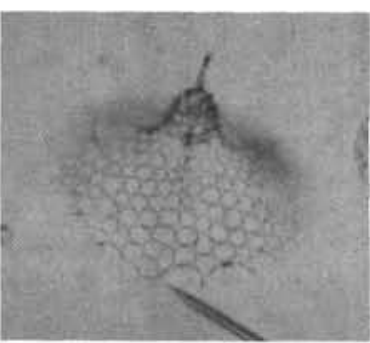

23

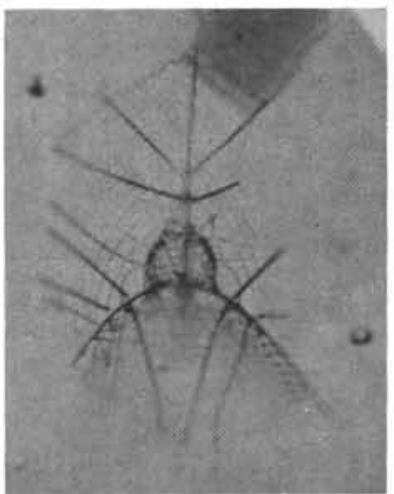

5

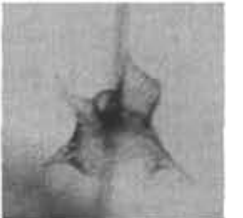

9

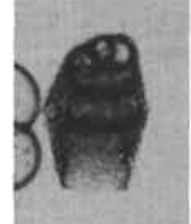

15

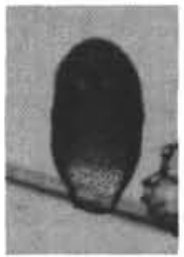

19

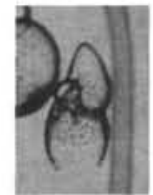

10

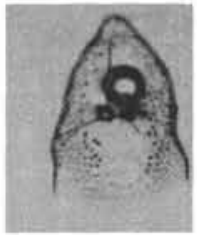

16

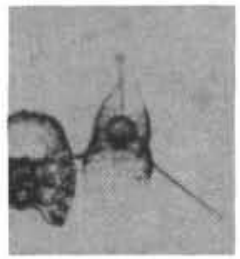

20

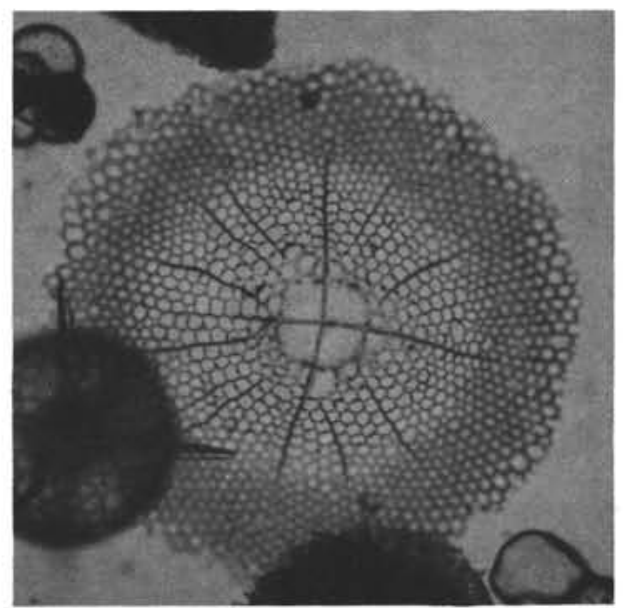

24 
PLATE 19

(Magnification $\times 150$ )

Figure 1

Figure 2

Figure 3

Figure 4

Figure 5

Figure 6

Figure 7

Figure 8

Figure 9

Figure 10

Figure 11

Figure 12

Figure 13

Figure 14

Figure 15

Figure 16

Figure 17

Figure 18

Figure $19 \mathrm{a}, \mathrm{b}$

Figure 20

Figure 21

Figure 22

Figure 23

Figure 24

Figure 25

Figure 26

Figure 27

Figure 28
Liriospyris reticulata (Ehrenberg); 262-1-2, 89-91. Liriospyris costata (Haeckel); 262-2-2, 94-96.

Liriospyris sp. 1; 262-1-2, 89-91.

Liriospyris sp. 2; 262-1, CC.

Tympanidium foliosum Haeckel; 262-1-2, 89-91.

Nephrospyris renilla Haeckel; 262-1-2, 89-91.

Giraffospyris laterispina Goll; 262-1-2, 89-91.

Ceratospyris sp. aff. C. sp. A; 262-1-4, 39-41.

Ceratospyris sp.; 262-1-2, 89-91.

Giraffospyris angulata (Haeckel); 262-1-2, 89-91.

Dendrospyris sp. aff. D. binapertonis Goll; 262-3-2, 84-86.

Tholospyris sp.; 262-1-2, 89-91.

Tholospyris scaphipes (Haeckel); 262-1-2, 31-33.

?Dendrospyris sp. aff. D. stabilis Goll; 262-1-2, 3133.

Zygocircus sp. 1 aff. Z. capulosus Popofsky; 262-12, 31-33.

Theoconus hertwigii (Haeckel); 262-1-2, 31-33.

Anthocyrtidium zanguebaricum (Ehrenberg); 2621-2, 89-91.

Tetracorethra tetracorethra (Haeckel), emend. Petrushevskaya; 262-1-2, 31-33.

Cantharospyris platybursa Haeckel; (a) 262-1-2, 89-91. (b) 262-1-2, 31-33.

Zygocircus capulosus Popofsky; 262-1-2, 89-91.

Lamprocyclas maritalis Haeckel; 262-1-2, 89-91.

Anthocyrtidium ophirense (Ehrenberg); 262-1-2, 31-33.

Zygocircus sp. 2 aff. Z. capulosus Popofsky; 262-12, 31-33.

Zygocircus sp. cf. Z. piscicaudatus Popofsky; 2621-2, 31-33.

Lamprocyrtis heteroporus (Hays); 262-2-2, 94-96.

Lamprocyrtis haysi Kling; 262-1-2, 89-91.

Theoconus junonis Haeckel; 262-1-2, 89-91.

Theocorythium trachelium (Ehrenberg); 262-2-2, 94-96. 
Plate 19

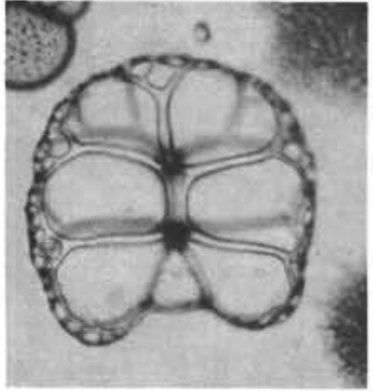

1

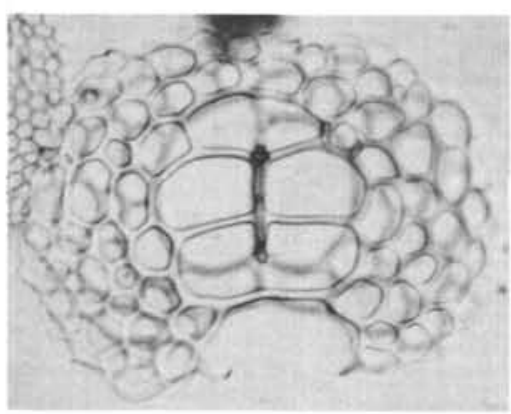

6

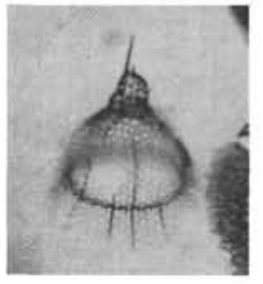

16

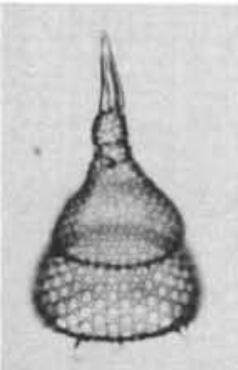

21

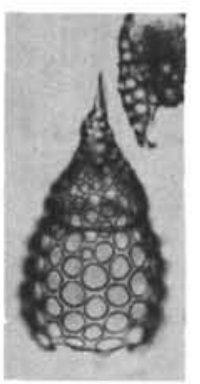

25

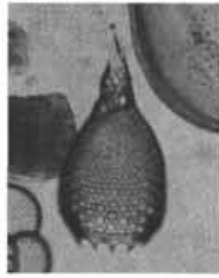

17

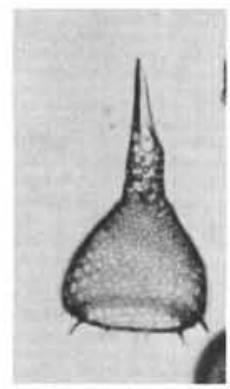

22

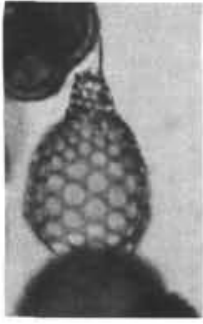

26

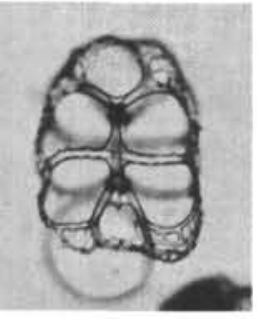

2

2

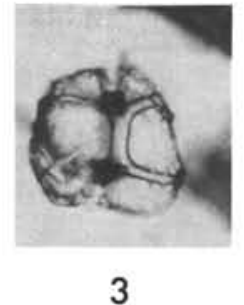

3
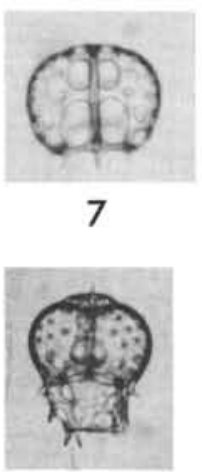

11

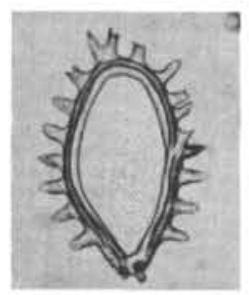

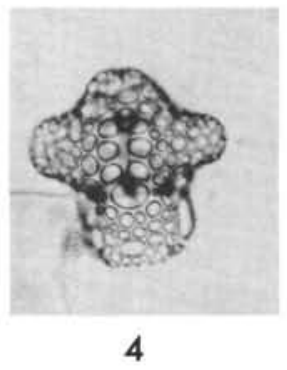

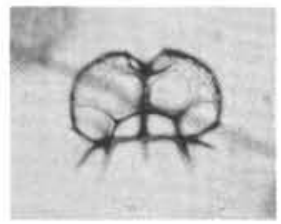

8

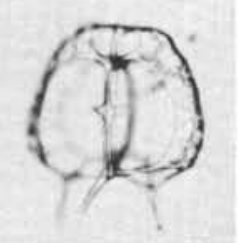

12

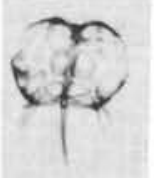

13

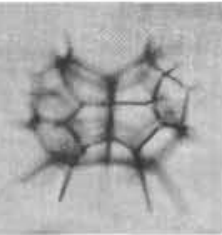

9

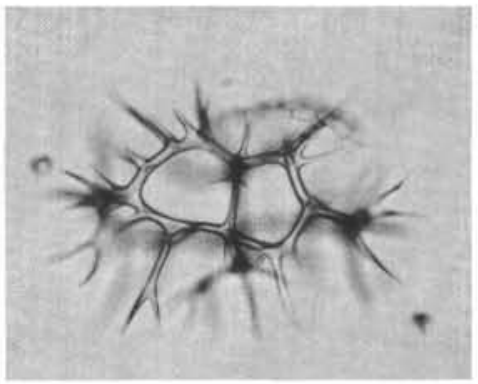

5

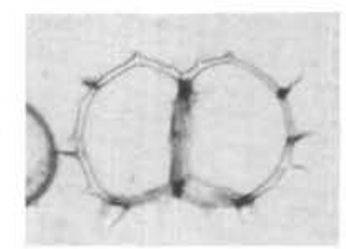

10
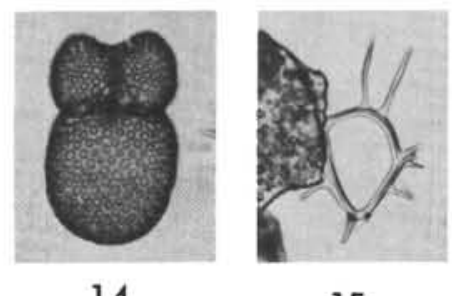

15

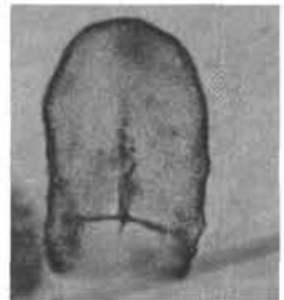

$19 a$

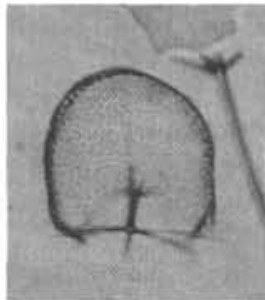

$19 b$

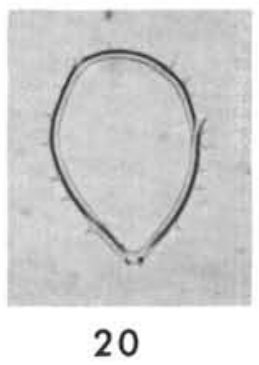

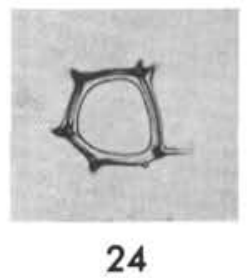

23

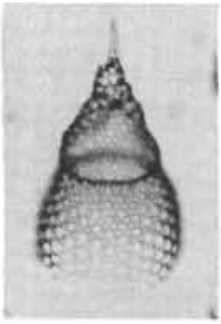

27

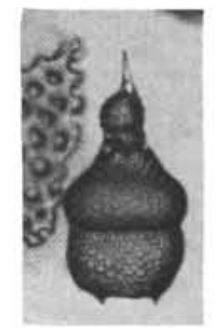

28 


\section{PLATE 20}

Siliceous Cretaceous Microfossils-Radiolaria?. Scanning electron photomicrographs provided by H. M. Bolli, Swiss Federal Institute of Technology, Zurich. All specimens from Sample 206-15, CC.

Figure $1 \quad \times 500$.

Figure $2 \times 500$.

Figure $3 \times 500$.

Figure $4 \times 450$.

Figure $5 \times 550$

Figure $6 \times 500$.

Figure $7 \times 1000$

Figure $8 \times 500$

Figure $9 \times 500$

Figure $10 \times 2500$.

Figure $11 \times 1000$.

Figure $12 \times 2500$. 
PLATE 20

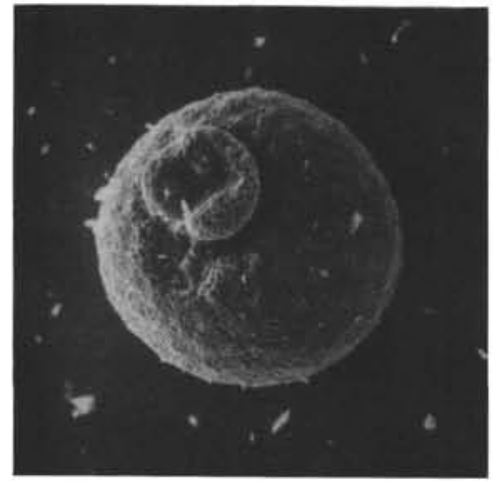

1

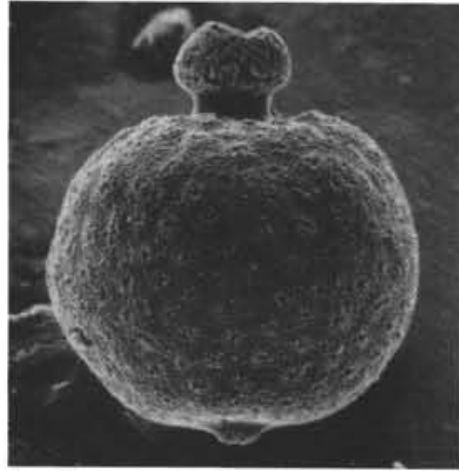

4

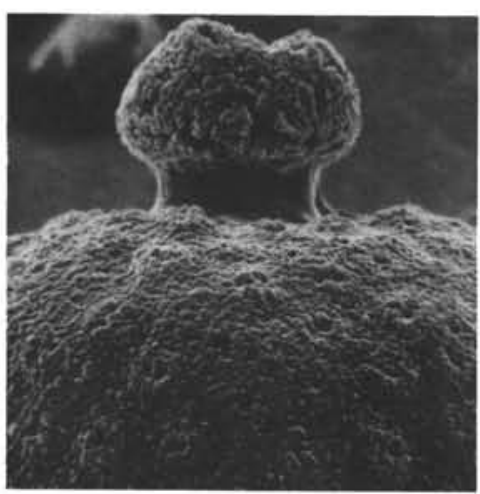

7

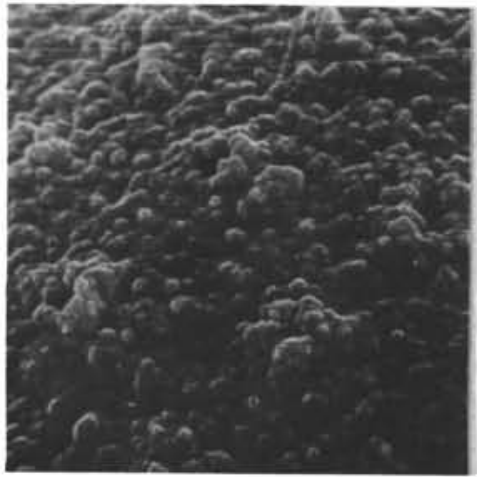

10

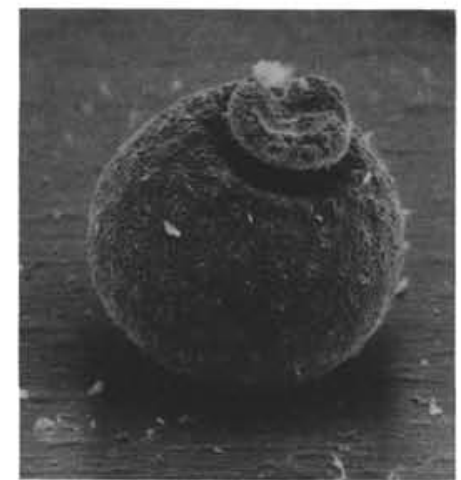

2

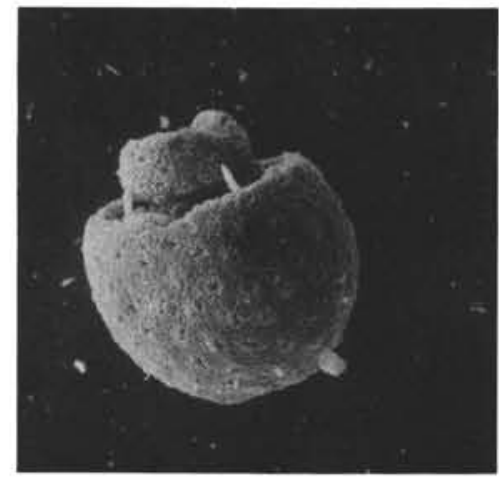

5

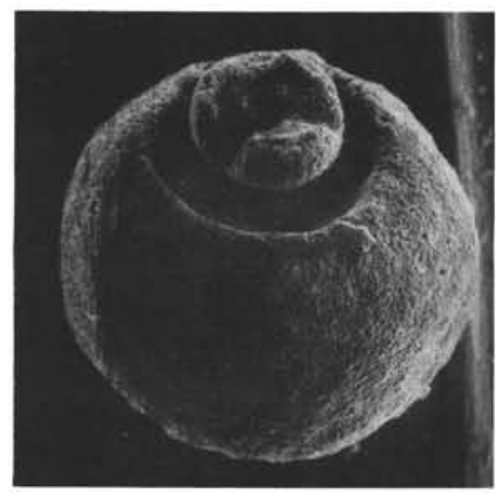

8

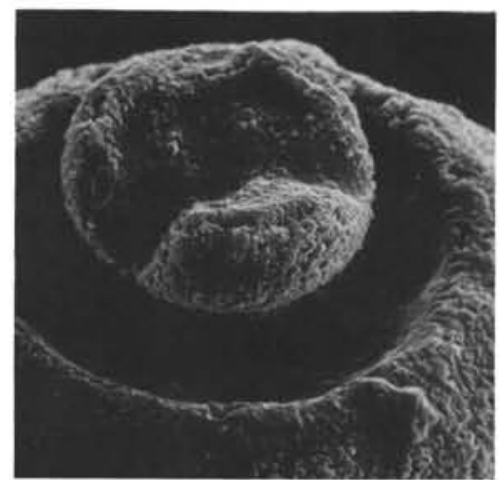

11

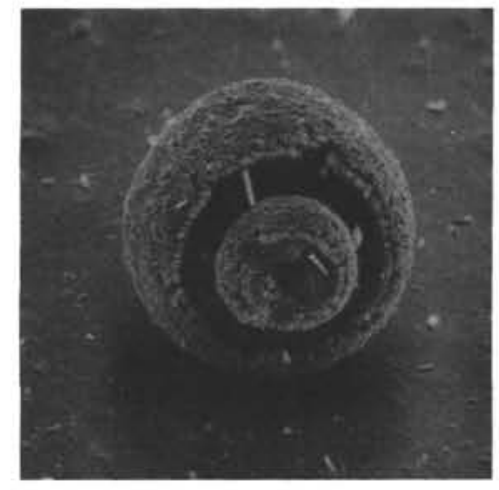

3

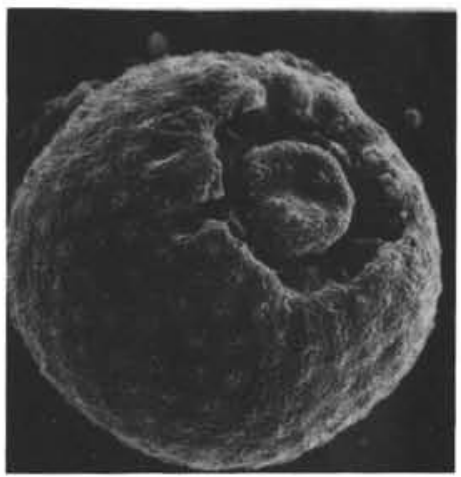

6

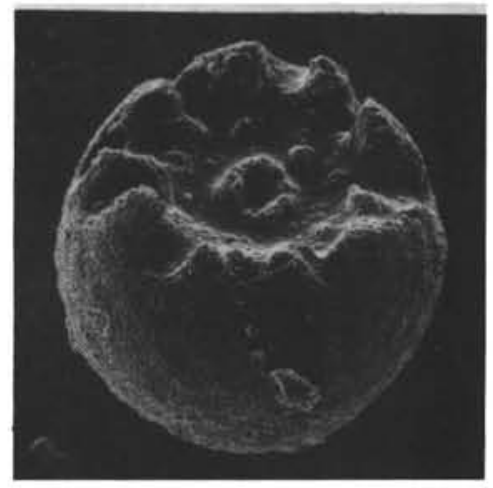

9

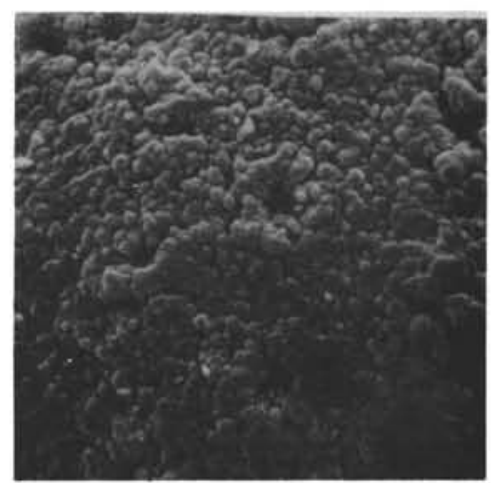

12 